UNIVERSITY OF SÃO PAULO

INSTITUTE OF PHYSICS

\title{
Desenvolvimento de um Software para Avaliação de Qualidade de Imagens Tomográficas usando o Phantom Catphan500 $^{\circledR}$
}

Daniel Vicente Vieira

Orientador: Paulo Roberto Costa

Banca Examinadora:

Prof. Dr. Paulo Roberto Costa (IFUSP)

Profa. Dra. Ana Maria Marques da Silva (PUCRS)

Prof. Dr. Homero Schiabel (EESC/USP)

Dissertação apresentada ao Instituto de Física da Universidade de São Paulo para a obtenção do título de Mestre em Ciência.

São Paulo 


\section{FICHA CATALOGRÁFICA \\ Preparada pelo Serviço de Biblioteca e Informação do Instituto de Física da Universidade de São Paulo}

Vieira, Daniel Vicente

Desenvolvimento de um software para avaliação de qualidade de imagens tomográficas usando o Phantom Catphan500 ${ }^{\circledR}$ : Development of a software for image quality assessment in computed tomography using the Catphan500® Phantom . São Paulo, 2016.

Dissertação (Mestrado) - Universidade de São Paulo. Instituto de Física. Depto. de Física Nuclear

Orientador: Prof. Dr. Paulo Roberto Costa

Área de Concentração: Física Médica.

Unitermos: 1. Tomografia; 2. Softwares; 3. Controle de qualidade. 
UNIVERSIDADE DE SÃO PAULO

INSTITUTO DE FÍSICA

\section{Development of a Software for Image Quality Assessment in Computed Tomography using the Catphan500 ${ }^{\circledR}$ Phantom}

Daniel Vicente Vieira

Advisor: Paulo Roberto Costa

Dissertation submitted to the Institute of Physics of the University of São Paulo for the Master of Science degree.

Examination Committee:

Prof. Dr. Paulo Roberto Costa (IFUSP)

Profa. Dra. Ana Maria Marques da Silva (PUCRS)

Prof. Dr. Homero Schiabel (EESC/USP)

São Paulo 



\section{ACKNOWLEDGMENTS}

Thanks to my family, Sueli, Luiz, Marina and Iann, for their unconditional support for the last two years.

I thank my advisor Paulo Costa for his undying patience and tireless work.

I want to thank the quality control team, source of much of the data of this project, and specially Denise Nersissian, unlimited source of answers.

I thank Juliana Martins for the delightful company sharing a room for two years, making a certain evils much more bearable.

I deeply thank Rafael de Campos for unknowingly (?) shaking off much of the inertia and boredom of my life.

Special thanks to Rafael Sumiya for our surprisingly long standing friendship, and the amazing trip to the seaside.

Thanks to all Demônios de Maxwell members for the wonderful time I had so far playing rugby, in spite of my long absences.

I thank CNPq and INCT for funding my work for two years under the project number 131667/2014-9. 


\section{Contents}

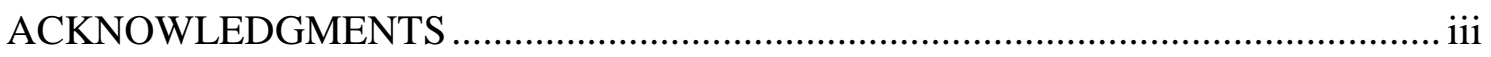

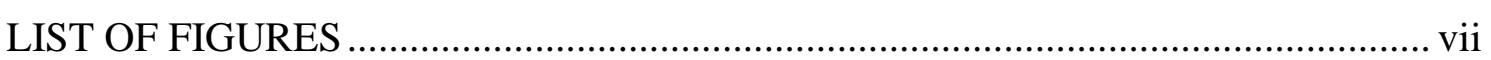

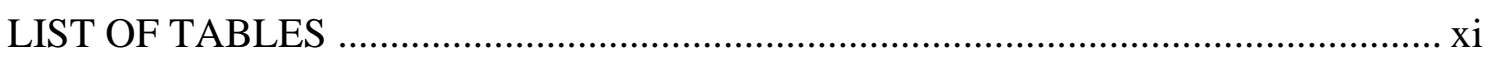

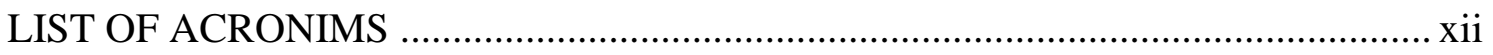

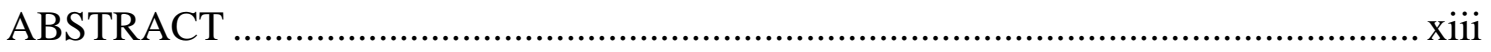

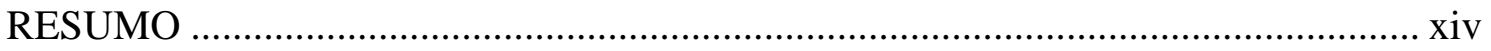

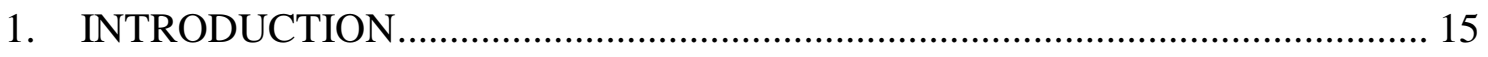

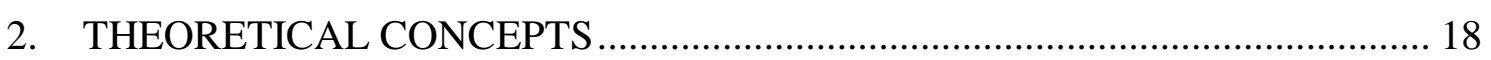

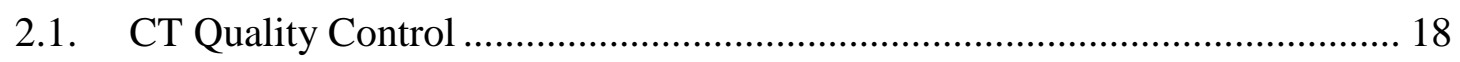

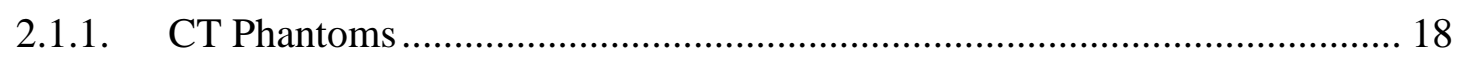

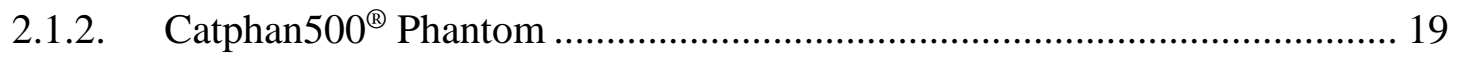

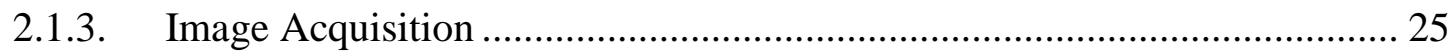

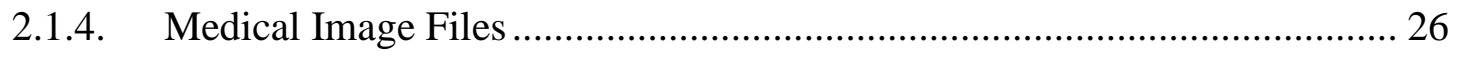

2.2. Mathematical Description of Imaging Systems ............................................ 27

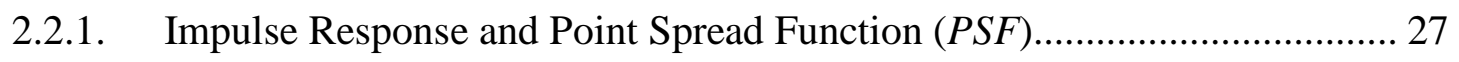

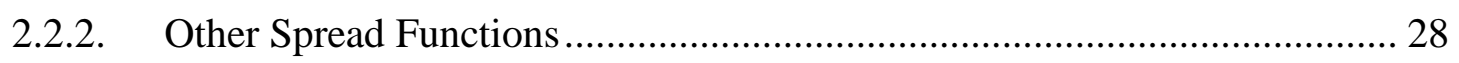

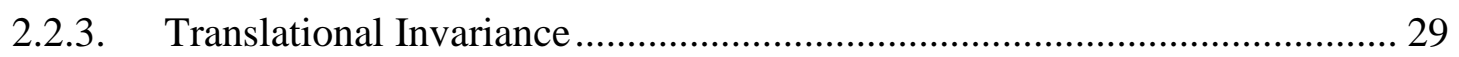

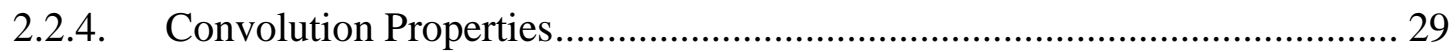

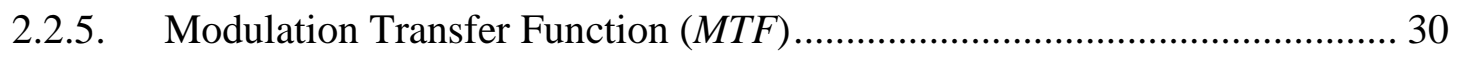

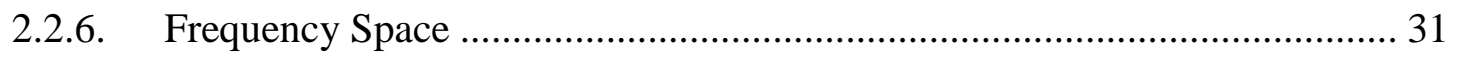

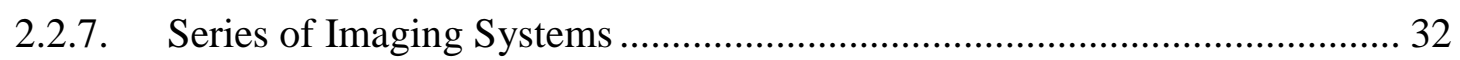

2.2.8. Noise and Noise Power Spectrum (NPS) ................................................. 32

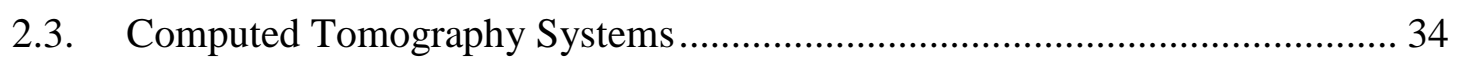

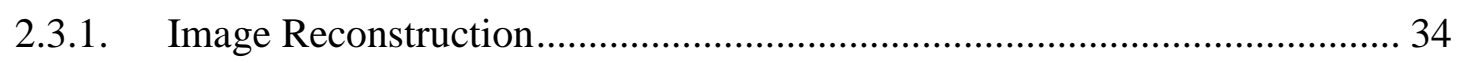

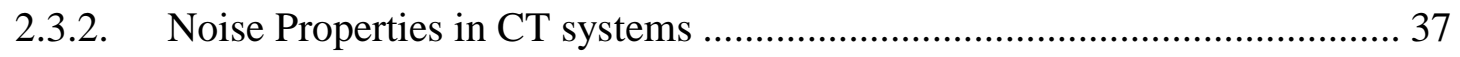

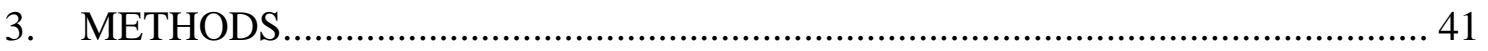

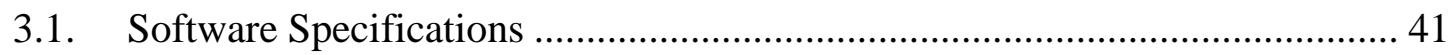

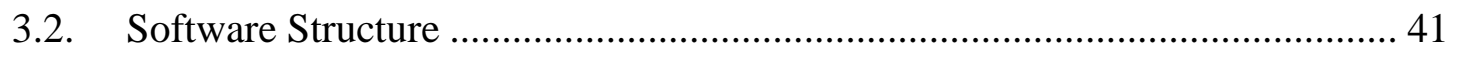

3.2.1. Sorting Images from the Header Information ........................................ 44

3.2.2. Identification of the Center and Radius of the Phantom ............................. 44

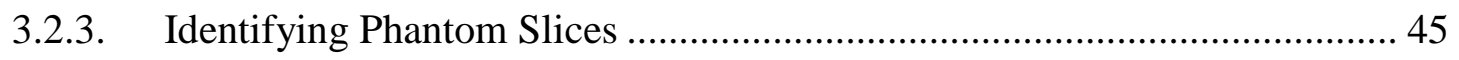

3.2.4. Modular Structure of the Software ............................................................... 47

3.3. Algorithm of Measurement of each Variable ............................................... 47

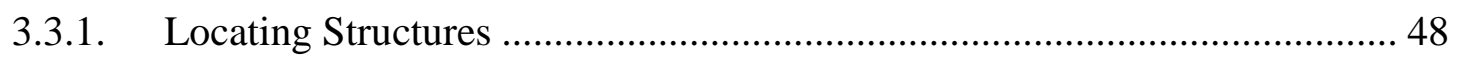

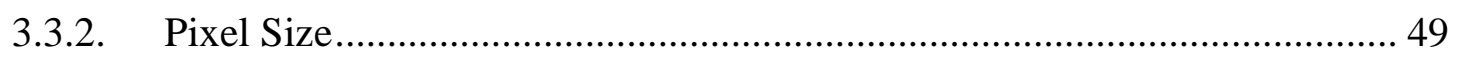

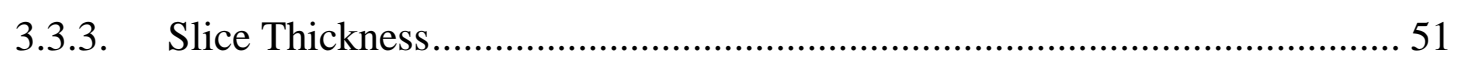




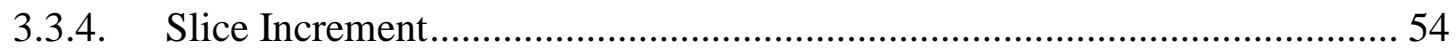

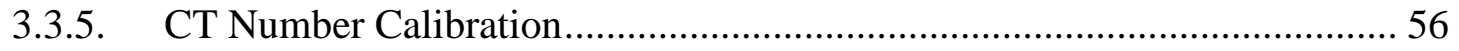

3.3.6. MTF Measurement and Cutoff Frequency Estimation............................... 57

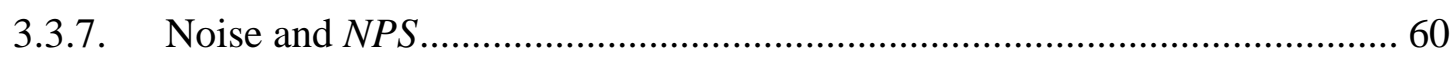

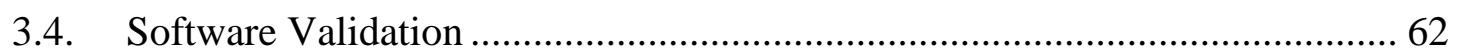

3.4.1. Pixel Size, Slice Thickness, Increment and CT Number Calibration .......... 63

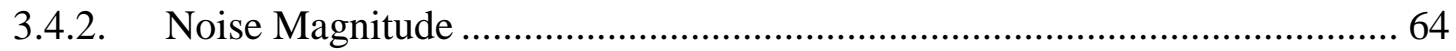

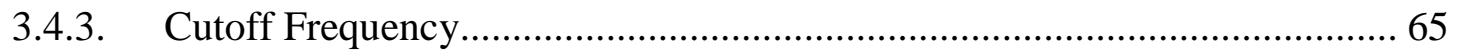

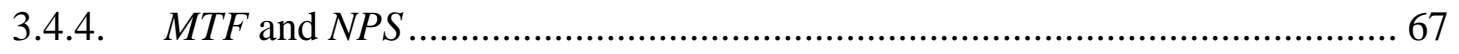

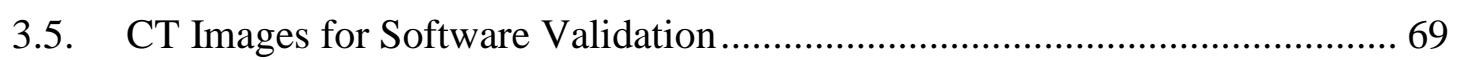

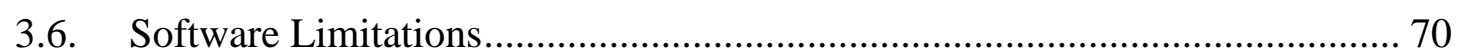

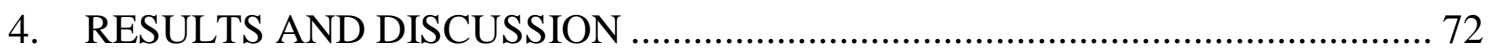

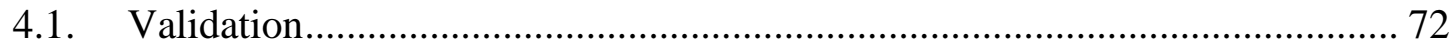

4.1.1. Pixel Size, Slice Thickness, Increment and CT Number Calibration .......... 72

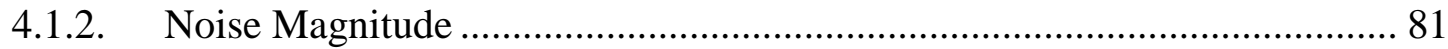

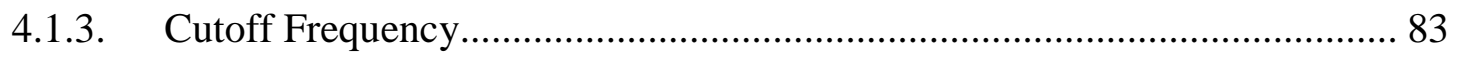

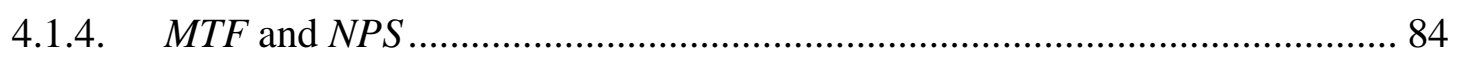

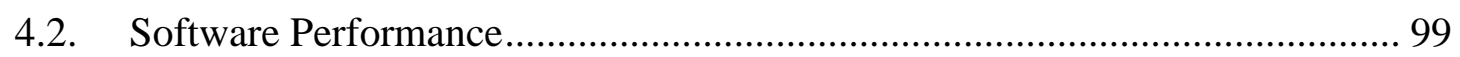

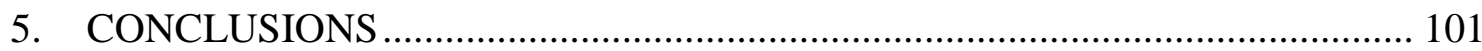

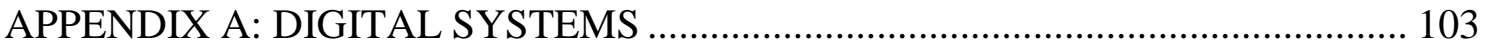

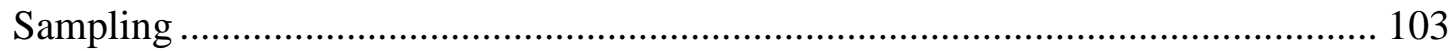

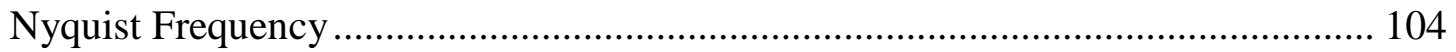

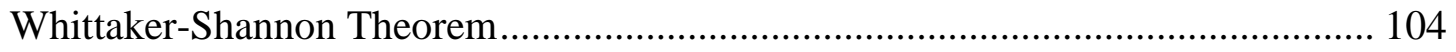

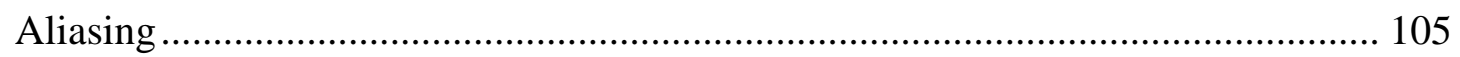

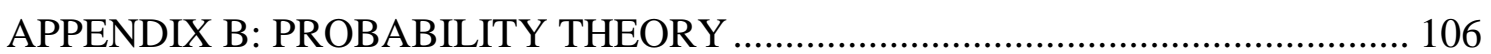

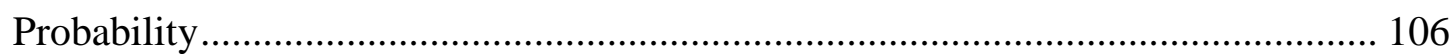

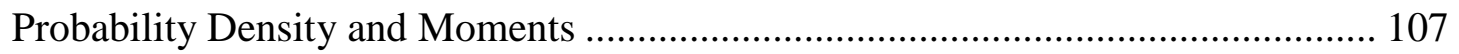

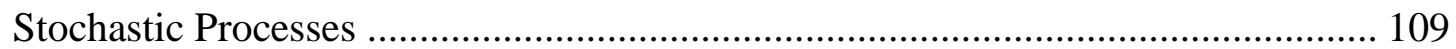

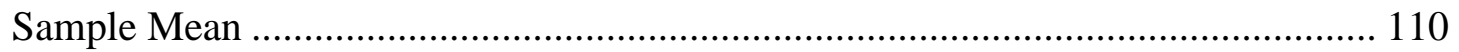

Autocorrelation and Stationary Processes ........................................................... 110

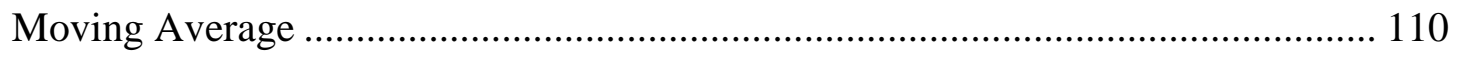

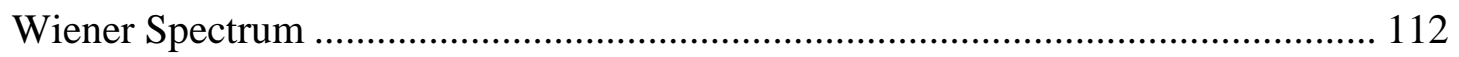

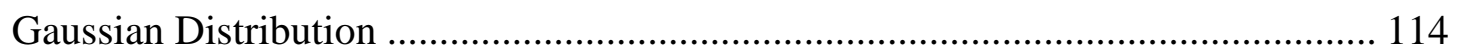

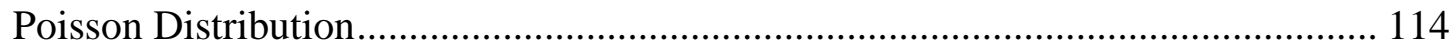

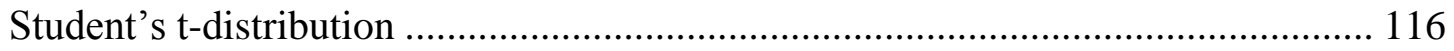

Chi-squared and Reduced Chi-squared Distribution ............................................ 118

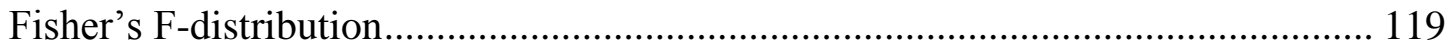




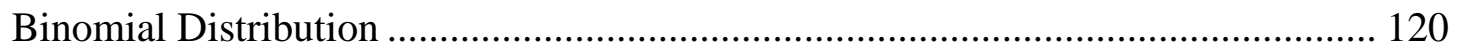

Test of Hypothesis and Error Types ................................................................... 121

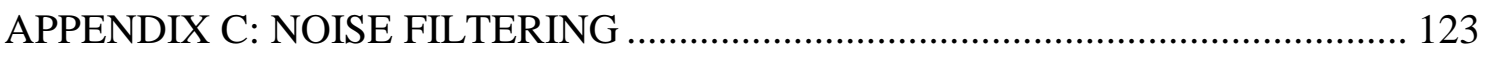

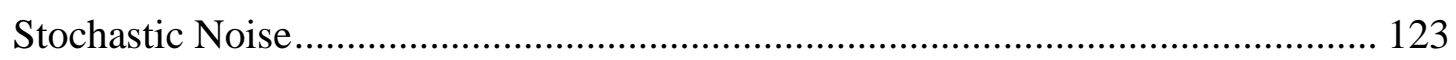

Optimized Filters .................................................................................. 124

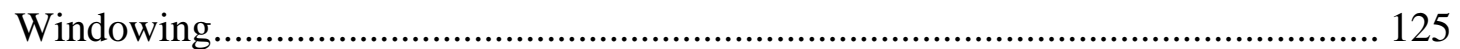

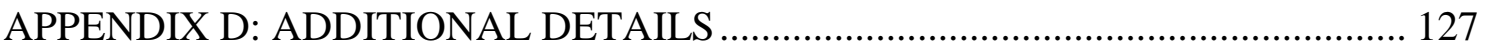

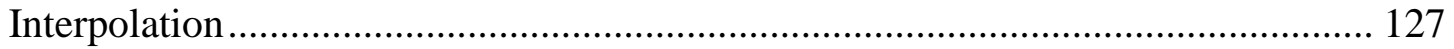

Slice Thickness 2\% Uncertainty .................................................................... 128

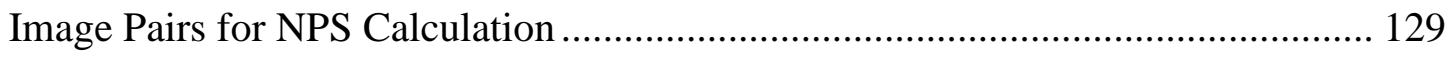

2-Dimensional Noise Power Spectrum ................................................................ 130

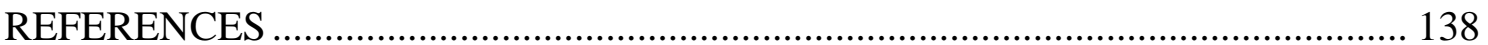




\section{LIST OF FIGURES}

Figure 1: (a) photo of Catphan $500^{\circledR}$ and its case, used as counterweight (courtesy of Nersissian, D. Y.); (b) scheme of the internal structure of Catphan500 ${ }^{\circledR 39}$ (source: Catphan $500^{\circledR}$ and $600^{\circledR}$ Manual).

Figure 2: (a) scheme of the CTP401 module of Catphan $500^{\circledR}$ phantom (source: Catphan $500^{\circledR}$ and $600^{\circledR}$ Manual). (b) CT image of the central slice of the CTP401 module.

Figure 3: (a) scheme of the CTP528 module of Catphan $500^{\circledR}$ phantom (source: Catphan $500^{\circledR}$ and $600^{\circledR}$ Manual). (b) CT image of the central slice of the CTP528 module. The second tungsten bead does not appear in this CT image because it is slightly dislocated in the z-axis.

22

Figure 4: (a) scheme of the CTP515 module of Catphan500 ${ }^{\circledR}$ phantom (source: Catphan $500^{\circledR}$ and $600^{\circledR}$ Manual). (b) CT image of the central slice of the CTP515 module. The grayscale was adjusted to better visualize the structures, which have very low contrast.

Figure 5: (a) scheme of the CTP486 module of Catphan $500^{\circledR}$ phantom (source: Catphan $500^{\circledR}$ and $600^{\circledR}$ Manual). (b) CT image of the central slice of the CTP486 module.

Figure 6: Catphan $500^{\circledR}$ in a CT scanner being aligned for an acquisition (courtesy of Nersissian, D. Y.). 26

Figure 7: This flowchart summarizes how the software works. Initially, a folder containing the phantom images is provided by the user. The software then organizes the images in subfolders by acquisition protocol, and in a selected subfolder organizes them in lists by phantom section. Then, each list is used by its respective module to calculate their variables of interest.

Figure 8: (a) Software interface, as it appears when the software is initialized. (b) Software interface at work, with its parts highlighted by the red boxes. 1) Box to select the folder containing the CT images to be analyzed. 2) The main commands of the software, used to manipulate the lists automatically and calculate the variables of interest. 3) List showing the images of the selected folder, with buttons to manually add images to the CTP401, MTF or Noise lists. 4) Log of operations previously performed. 5) Area for visualization of the CT images. 6) Information about the selected image extracted from the DICOM header. 7) Lists CTP401, MTF and Noise, with their respective Delete buttons.

Figure 9: Image corresponding the Catphan $500^{\circledR}$ CTP401 central slice. The red boxes highlight the ROI's selected where the alignment pins are expected to be found. The yellow boxes highlight the regions used to calculate the background. 46

Figure 10: CT image showing the central slice of the module CTP401 of the Catphan $500^{\circledR}$ phantom. With this image, the software must locate the 4 cylinders of air and Teflon, calculate the distances between them, and use it to calculate the pixel size.

50

Figure 11: This picture illustrates the procedure to select a region containing the Teflon cylinder of Catphan $500^{\circledR}$. The software first calculates the center of the phantom c (by the algorithm described in 3.2.2). Then it is added to the relative position of the cylinder prel (built into the software, as explained in 3.3.1). This defines a point $\mathrm{p}$, which is approximately where the cylinder is located. The software then selects a ROI around this point. 50

Figure 12: (a) CT image of the central part of the module CTP401 of the Catphan $500^{\circledR}$ phantom. The red square depicts the ROI previously defined containing the Teflon 
cylinder. The exact position corresponding to this cylinder in the image, $\mathbf{p} 2$, is then obtained by the point of maxima in this ROI (because the structure is lighter than the background; if it were darker it would be the point of minima). (b) The procedure depicted in Figure 11 and Figure 12a is repeated for the other 3 cylinders, obtaining p1, p3 and p4. The software then measures the distances between each pair, illustrated in light blue.

Figure 13: (a) CT image of the central part of the module CTP401 of the Catphan phantom. The procedure to define a ROI containing a ramp (solid red rectangle) is analogous to the procedure depicted in Figure 11 and Figure 12. A profile is then traced parallel to the ramp, crossing its point of maxima (light blue line). This procedure is repeated for each of the 4 ramps (dashed red rectangles). (b) Profile of the ramp shown in the solid red square of Figure 13a, subtracted of the background. The red line is a Gaussian function fitted to the profile. The green lines indicate the total height of the peak $\left(2 h_{m}\right)$ and the full width at half maxima (FWHM). The FWHM is used to calculate the slice thickness.

Figure 14: (a) CT image of the central slice of the CTP401 module of Catphan $500^{\circledR}$. (b) CT image of another slice of the CTP401 module of Catphan500 ${ }^{\circledR}$. This is not the central slice of the module: notice the clockwise dislocation of the 4 ramps (highlighted in the red boxes) when compared to (a). 55

Figure 15: ramp profiles of 2 consecutive images of CTP401 module, with the center of each peak highlighted by the dashed line and the distance between them represented by $\Delta \mathrm{x}$. 56

Figure 16: CT image of the central part of the module CTP401 of the Catphan phantom; the red boxes highlight the 4 cylinders used in the calibration of the CT number. Clockwise from the top, the material of each cylinder is: air, Teflon, acrylic and LPDE.

Figure 17: amplified CT image of the CTP528 module of the Catphan500 ${ }^{\circledR}$ phantom. The blank red box represents the ROI selected around the tungsten bead. The striped red box represents the ROI used to calculate the background.

Figure 18: CT image representing the CTP528 Catphan $500^{\circledR}$ module, illustrating the process of obtaining the $\mathrm{QC}$ estimation of the cutoff frequency ( $\mathrm{f}_{\mathrm{QC}}$, in yellow). Following the clockwise red arrow, the last pattern where the bars are visually still distinguishable is found and its spatial frequency $f_{L D}$ (in red) is recorded. In the next pattern, of spatial frequency $f_{\text {next }}$ (in blue), the bars are not distinguishable anymore. The actual estimative of the cutoff frequency is between these two frequencies and has constant distribution.

Figure 19: Example of measured NPS, using a Phillips Brilliance16 CT scanner, in axial mode and selecting $1.5 \mathrm{~mm}$ of slice thickness. (a) 2D NPS. (b) NPS expressed onedimensionally (red line) and fitted as a function of the MTF (blue points).

Figure 20: Slice thickness obtained by the software (squares) and the QC (triangles) for each protocol, sorted by scanner (different colors).

Figure 21: Percentual difference between the software and the QC slice thickness results in each protocol, sorted by scanner (different colors).

Figure 22: Student's t value for slice thickness for each protocol, sorted by scanner (different colors). The red dashed lines represent the acceptance interval. Only protocol 19 is outside the acceptance interval.

Figure 23: Slice increment obtained by the software (squares) and the QC (triangles) for each protocol, sorted by scanner (different colors).

Figure 24: Percentual difference between the software and the QC slice increment results in each protocol, sorted by scanner (different colors). 
Figure 25: Student's t value for slice increment for each protocol. The QC does not measure it at all protocols. The red dashed lines represent the acceptance interval. All values are within the acceptance interval.

Figure 26: Pixel size obtained by the software (squares) and the QC (triangles) for each

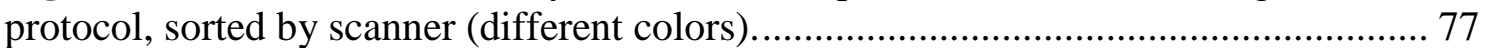

Figure 27: Percentual difference between the software and the QC pixel size results in

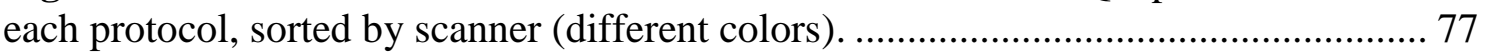
Figure 28: Student's t value for pixel size. The QC does not measure it at all protocols. The red dashed lines represent the acceptance interval. All values are within the acceptance interval. 78

Figure 29: Linear coefficient of the CT number calibration obtained by the software (squares) and the QC (triangles) for each protocol, sorted by scanner (different colors). 78

Figure 30: Student's t value for the linear coefficient of the fit for CT number calibration. The red dashed lines represent the acceptance interval. Protocols 6, 7, 9 and 27 are out of the interval.

Figure 31: Angular coefficient of the CT number calibration obtained by the software (squares) and the QC (triangles) for each protocol, sorted by scanner (different colors).

Figure 32: Student's t value for the angular coefficient of the fit for CT number calibration. The red dashed lines represent the acceptance interval. All values are within the acceptance interval. 80

Figure 33: Noise magnitude obtained by the software (squares) and the QC (triangles) for each protocol, sorted by scanner (different colors).

Figure 34: Fisher F value comparing the noise magnitude of the software and the QC report, sorted by scanner (different colors). The red dashed lines represent the acceptance interval. Protocols 15 and 16 are outside the acceptance interval (16 is not represented, it is 0.47 , much below the scale of this graphic).

Figure 35: cutoff frequency estimated by the software (squares, $\mathrm{f}_{\mathrm{c}}$ ), frequency at $10 \%$ of the MTF (circles, $\mathrm{f}_{10 \%}$ ) and the cutoff frequency estimated by the QC (triangles, $\mathrm{f}_{\mathrm{QC}}$ ) for each protocol, sorted by scanner (different colors).

Figure 36: (a) MTF fitted by an illustrative Gaussian curve. (b) NPS measured at protocol 2 (Toshiba Aquillion, axial mode, 1.0mm slice thickness, 240mm FOV) fitted as a function of the MTF. 86

Figure 37: (a) MTF fitted by an illustrative Gaussian curve. (b) NPS measured at protocol 6 (Phillips Brilliance16, axial mode, 1.5mm slice thickness, 250mm FOV) fitted as a function of the MTF.

Figure 38: (a) MTF fitted by an illustrative Gaussian curve. (b) NPS measured at protocol 8 (Phillips Brilliance40, axial mode, 1.25mm slice thickness, 250mm FOV) fitted as a function of the MTF. 88 Figure 39: (a) MTF fitted by an illustrative Gaussian curve. (b) NPS measured at protocol 11 (Phillips Brilliance BigBore, axial mode, $1.5 \mathrm{~mm}$ slice thickness, $250 \mathrm{~mm}$ FOV) fitted as a function of the MTF.

Figure 40: (a) MTF fitted by an illustrative Gaussian curve. (b) NPS measured at protocol 15 (GE Discovery690, axial mode, $1.25 \mathrm{~mm}$ slice thickness, 250mm FOV) fitted as a function of the MTF.

Figure 41: (a) MTF fitted by an illustrative Gaussian curve. (b) NPS measured at protocol 16 (GE Discovery690, axial mode, $2.5 \mathrm{~mm}$ slice thickness, 250mm FOV) fitted as a function of the MTF. 
Figure 42: (a) MTF fitted by an illustrative Gaussian curve. (b) NPS measured at protocol 17 (GE Discovery750, axial mode, 1.25mm slice thickness, 250mm FOV) fitted as a function of the MTF.

Figure 43: (a) MTF fitted by an illustrative Gaussian curve. (b) NPS measured at protocol 18 (GE Discovery750, helical mode, 2.5mm slice thickness, 250mm FOV) fitted as a function of the MTF.

Figure 44: (a) MTF fitted by an illustrative Gaussian curve. (b) NPS measured at protocol 21 (Siemens Somatom Emotion16, 1.2mm slice thickness, 250mm FOV) fitted as a function of the MTF. 94

Figure 45: (a) MTF fitted by an illustrative Gaussian curve. (b) NPS measured at protocol 22 (GE LightspeedUltra, axial mode, $1.25 \mathrm{~mm}$ slice thickness, 240mm FOV) fitted as a function of the MTF.

Figure 46: (a) MTF fitted by an illustrative Gaussian curve. (b) NPS measured at protocol 24 (GE LightspeedUltra, axial mode, $2.5 \mathrm{~mm}$ slice thickness, 250mm FOV) fitted as a function of the MTF. 96

Figure 47: (a) MTF fitted by an illustrative Gaussian curve. (b) NPS measured at protocol 25 (Phillips Brilliance64, axial mode, 1.25mm slice thickness, 250mm FOV) fitted as a function of the MTF.

Figure 48: (a) MTF fitted by an illustrative Gaussian curve. (b) NPS measured at protocol 26 (Phillips Brilliance64, axial mode, 2.5mm slice thickness, 250mm FOV) fitted as a function of the MTF. 98

Figure 49: 2D NPS measured at protocol 2 (Toshiba Aquillion, axial mode, $1.0 \mathrm{~mm}$ slice thickness, 240mm FOV). 130

Figure 50: 2D NPS measured at protocol 6 (Phillips Brilliance16, axial mode, $1.5 \mathrm{~mm}$ slice thickness, 250mm FOV). 131

Figure 51: 2D NPS measured at protocol 8 (Phillips Brilliance40, axial mode, $1.25 \mathrm{~mm}$ slice thickness, 250mm FOV).

Figure 52: 2D NPS measured at protocol 11 (Phillips Brilliance BigBore, axial mode, $1.5 \mathrm{~mm}$ slice thickness, $250 \mathrm{~mm}$ FOV). 132

Figure 53: 2D NPS measured at protocol 15 (GE Discovery690, axial mode, $1.25 \mathrm{~mm}$ slice thickness, 250mm FOV). 132

Figure 54 2D NPS measured at protocol 16 (GE Discovery690, axial mode, 2.5mm slice thickness, 250mm FOV).

Figure 55: 2D NPS measured at protocol 17 (GE Discovery750, axial mode, $1.25 \mathrm{~mm}$ slice thickness, 250mm FOV). 133

Figure 56: 2D NPS measured at protocol 18 (GE Discovery750, helical mode, $2.5 \mathrm{~mm}$ slice thickness, 250mm FOV). 134

Figure 57: 2D NPS measured at protocol 21 (Siemens Somatom Emotion16, 1.2mm slice thickness, 250mm FOV). 134 Figure 58: 2D NPS measured at protocol 22 (GE LightspeedUltra, axial mode, $1.25 \mathrm{~mm}$ slice thickness, 240mm FOV). 135

Figure 59: 2D NPS measured at protocol 24 (GE LightspeedUltra, axial mode, $2.5 \mathrm{~mm}$ slice thickness, 250mm FOV). 135

Figure 60: 2D NPS measured at protocol 25 (Phillips Brilliance64, axial mode, $1.25 \mathrm{~mm}$ slice thickness, 250mm FOV). 136 Figure 61: 2D NPS measured at protocol 26 (Phillips Brilliance64, axial mode, $2.5 \mathrm{~mm}$ slice thickness, 250mm FOV). 136 


\section{LIST OF TABLES}

Table 1: scanners used to test the software, separated by hospital, manufacturer and model.

Table 2: List of the protocols used to validate the software. The numbering in the first column is the same used in the graphs in the next subsections. The second column shows the scanner model and manufacturer. The third column presents the acquisition mode (axial or helical). The fourth column presents the reconstruction algorithm used. The fifth column gives the name of the applied filter (as it is in the DICOM header). The sixth column presents the selected nominal slice thickness (in $\mathrm{mm}$ ). The last column presents the DFOV selected (also in $\mathrm{mm}$ ). Each group of protocols with the same scanner is separated from the others by a bold line. An asterisk beside the protocol number indicates this is one of the protocols where the calculation of the NPS was possible.

Table 3: Time spent per image by the function "Sort" measured for each test folder. Each test folder contained images of a different scanner, as presented in the first column. The last folder contained all the images from the previous folder. The last line of the table presents the resulting average (in ms/image) with the standard deviation. 100 Table 4: Time spent per image by the function "Identify" measured for each test folder. Each test folder contained images of a different scanner, as presented in the first column. The last line of the table presents the resulting average (in ms/image) with the standard deviation. 100

Table 5: Protocols used to quantify the noise influence on the slice thickness uncertainty. The last column shows the relative uncertainties obtained for the slice thickness, and the last line shows the average of all of them. 129 


\section{LIST OF ACRONIMS}

AAPM - American Association of Physicists in Medicine

ACR - American College of Radiology

AEC - Automatic Exposure Control

ANVISA - Agência Nacional de Vigilância Sanitária

CT - Computed Tomography

CTDI - Computed Tomography Dose Index

DFOV - Display Field of View

DICOM - Digital Imaging and Communications in Medicine

ESF - Edge Spread Function

FBP - Filtered Back-projection

IAEA - International Atomic Energy Agency

LSF - Line Spread Function

MTF - Modulation Transfer Function

NEMA - National Electrical Manufacturers Association

NPS - Noise Power Spectrum

PSF - Point Spread Function

ROI - Region of Interest

SFOV - Scan Field of View

TCM - Tube Current Modulation 


\section{ABSTRACT}

Since the introduction of the CT scanner as a diagnostic imaging modality, the scientific community has seen new and more complex CT technologies. These improvements brought the need for new and improved techniques to evaluate the safety and performance of these scanners. Nowadays, the interpretation of images generated during the implementation of CT quality control procedures are done visually in much of the cases. Therefore, it is slow and partially subjective. In this work, a software was written in MatLab to process images of the Catphan500 CT phantom, in order to improve the CT quality control workflow and its accuracy. The software evaluate the slice thickness, slice increment, and pixel size, calculates the CT number linearity, and assesses the Modulation Transfer Function (MTF), the noise and the Noise Power Spectrum (NPS). Image sets of the phantom were obtained from 10 different scanners using 27 different protocols in order to validate the software. Comparative results correlating the software output and corresponding data previously obtained by the current quality control program routine were used to conduct this validation. For this comparison, two statistical tests were employed: the Student's t-test (for slice thickness, slice increment, pixel size, and the coefficients of the CT number linearity evaluation, with a chosen pvalue of 0.01 ) and the Fisher F-test (for the noise, with chosen p-value of 0.05). The functions MTF and NPS are not currently measured by the quality control routine, so there was no previous result for comparison. Instead, the NPS was fitted as a function of the $M T F$ (using the theoretical relationship between both functions) and the quality of the fit was evaluated using the reduced chi-square. From $101 \mathrm{t}$ values and $25 \mathrm{~F}$ values calculated, 2 and 1 were outside the acceptance interval, respectively. This result agrees with the chosen p-values, and therefore the software results are in good agreement with the traditional quality control routine results. The fits of NPS and MTF presented large uncertainties in the fitting parameters (uncertainties of the same order of magnitude as the parameters themselves). However, the reduced chi-square evaluation indicates a good fit (with the exception of one fit, which showed an anomaly on the measured NPS and was unconsidered). Therefore, the obtained MTF and NPS were in agreement with the theoretical expectations.

Keywords: computed tomography, software, quality control 


\section{RESUMO}

Desde a invenção da tomografia computadorizada (CT) nos anos 70, toda década trouxe novas tecnologias para esta modalidade. Com estes avanços, também surgiu a necessidade de novas e melhores técnicas de avaliação de desempenho e segurança dos equipamentos de CT. Hoje, o controle de qualidade de equipamentos de CT é, em grande parte, feito manualmente. Portanto, é lento e, em parte, subjetivo. Neste trabalho, um software foi escrito em MatLab ${ }^{\circledR}$ para processar imagens do phantom de CT Catphan $500^{\circledR}$, aperfeiçoando a rotina do programa de controle de qualidade de CT. Com pouca interferência do usuário, o software mede a espessura de corte, incremento entre cortes e tamanho de pixel, avalia a linearidade do número CT, estima a Função Transferência de Modulação (MTF), o ruído e o Espectro de Potência do Ruído (NPS). Para a validação do software, conjuntos de imagens do phantom foram obtidas em 10 equipamentos de CT diferentes, com 27 protocolos diferentes. Cada conjunto foi analisado pelo software, e os resultados obtidos foram comparados aos resultados previamente obtidos pela rotina normal do programa controle de qualidade. Para essa comparação, dois testes de hipótese foram empregados: o teste t de Student (para os valores de espessura de corte, incremento entre cortes, tamanho de pixel e os coeficientes da avaliação de linearidade do número CT, adotando um valor-p de 0,01) e o teste F de Fisher (para o ruído, valor-p de 0,05). As funções MTF e NPS atualmente não são medidas na rotina do controle de qualidade, portanto não há resultado prévio para fazer esta comparação. Ao invés disso, o NPS foi ajustado em função da MTF (através da relação teórica que há entre os dois) e a qualidade do ajuste foi avaliada pelo teste de qui-quadrado. Dos 101 valores de t e 25 valores de F calculados, 2 e 1 respectivamente estavam fora do intervalo de aceitação. Este resultado está de acordo com os valores-p escolhidos e, portanto, os resultados obtidos pelo software estão de acordo com os resultados da rotina de controle de qualidade convencional. Os ajustes de NPS e MTF obtiveram incertezas grandes nos parâmetros de ajuste (incertezas da mesma ordem de grandeza dos próprios parâmetros). Porém, a avaliação do quiquadrado reduzido indica que os ajustes foram aceitáveis (com exceção de um, que mostrou uma anomalia no NPS medido e foi desconsiderado). Portanto, o NPS e MTF obtidos estão de acordo com a expectativa teórica.

Palavras-chave: tomografia, software, controle de qualidade 


\section{INTRODUCTION}

In 1917, Radon ${ }^{1 ; 2}$ proved mathematically that an image of an unknown object could be produced having infinite projections through the object, but at the time there was not enough computational power available to create a real calculation device for conducing the implementation of his theorem. It was only at the seventies that, with the improvement of computers, that the computed tomography (CT) became possible. This invention led Godfrey Hounsfield and Allan Cormack to win the Nobel Prize of Physiology and Medicine in $1979^{3}$.

The first CT scanner, the EMI Mark I ${ }^{1}$, was used clinically for the first time in 1972. The system worked using a single detector and the $\mathrm{x}$-rays were emitted by a strongly collimated x-ray tube (pencil beam). The produced images had 80x80 pixels (the side of each pixel was $3.0 \mathrm{~mm}$ ), and each slice had $13 \mathrm{~mm}$ thick. The hardware required 4.5 minutes to acquire the transmission data and 1.5 minutes to reconstruct one slice. These characteristics made them only useful for head scans ${ }^{4}$.

Since then, the CT technology has evolved swiftly ${ }^{5}$. The pencil beam was replaced by a fan beam, and the single detector by an array of detectors (acquiring a whole projection instantly and reducing acquisition time per slice to 20 seconds, enough to make a slice in a single breath hold) ${ }^{1}$. In the eighties, the cumbersome high voltage cables were substituted by the slip ring technology $5 ; 6$, making possible image acquisitions during only 3 seconds due to the continuous rotation of the x-ray tube-detectors system. The slip ring also made possible the introduction of the helical ${ }^{7}$ acquisition mode. In this kind of acquisition, the tube and detectors rotate continuously around the patient and the table moves along the z-axis simultaneously. In the nineties, improvements on the detector technology led to the multi-slice $\mathrm{CT}^{5 ; 8-11}$. In this new technology, the linear array of detectors was substituted by a matrix of detectors, acquiring multiple slices in a single rotation of the tube-detectors system. Improvements in computers allowed reconstruction to be done simultaneously to the scanning, and be observed in real time (allowing medical interventional procedures guided by CT images). The Automatic Exposure Control (AEC) ${ }^{12 ;} 13$ technology, also called Tube Current Modulation (TCM) in the specific context of $\mathrm{CT}$, led to reduction in the dose delivered to patients. Modern scanners produce real time images in matrices of $512 \times 512$ pixels $(0.5 \mathrm{~mm}$ pixel side). They can acquire up to 320 slices per rotation with thicknesses as low as $0.4 \mathrm{~mm}$ and each rotation lasts less than 0.5 seconds. 
Because of all these advances, quality control (QC) techniques needed to be reviewed and new tools to be developed ${ }^{14-17}$. Just after the introduction of the first CT scanner, the first CT dosimetry and image quality phantoms were invented ${ }^{18 ;}{ }^{19}$. To address dosimetric questions arisen from the new geometry, the CT dose index (CTDI) was created $^{20}$. Attempting to more directly relate the CTDI with the patient dose, other dose metrics were created such as the DLP ${ }^{21}$ (Dose-Length Product) and the $\operatorname{SSDE}^{22}$ (SizeSpecific Dose Estimate), and even today these are subject of continuous research ${ }^{23}$, as well as the relationship between dose and image quality ${ }^{24 ; 25}$. The assessment of the AEC system performance required the production of a special conical phantom ${ }^{26}$. In the present work, a computational QC tool was developed: a software to evaluate image quality of CT systems. The software allows partially automating the CT quality control process, by the assessment of image parameters obtained from specific sets of images acquired from a phantom. The software also improves on it by employing image quality metrics such as the modulation transfer function $(M T F)$ and the noise power spectrum (NPS). These functions have been known for some time from the theory of linear systems ${ }^{27}$, but only recently ${ }^{28-30}$ they started being applied to radiology due to the experimental complexity on measuring them.

Chapter 2 presents the relevant theoretical concepts needed as a background to this work. Section 2.1 provides some details about the quality control in CT, presenting one of the commercial phantoms adopted for this purpose (the Catphan500) and briefly explaining the image acquisition and manipulation process. Section 2.2 exposes the mathematical basis of imaging systems, defining the basic metrics used in the evaluation of image quality. Section 2.3 employs the formalism of the previous section to CT systems.

Chapter 3 presents the mathematical methods employed in this work. Section 3.2 describes the structure of the developed computer program and the basic function of each part. Section 3.3 presents the details of the algorithm developed to measure each variable of interest. Section 3.4 describes the process of validating the developed software, employing statistical tests of hypothesis. These tests allow comparing results obtained from the application of the software to the results previously obtained by traditional quality control routine based on human observers. Finally, section 3.5 explains the limits of applicability of the software. 
Chapter 4 presents the results of the validation process described in the previous chapter, and discusses the obtained results. Finally, Chapter 5 closes this work with the attained conclusions and suggestions for future works. 


\section{THEORETICAL CONCEPTS}

\subsection{CT Quality Control}

The CT scanners, as any other medical equipment, must be periodically evaluated by a quality control program ${ }^{31}$. These programs are composed by tests that have three major goals: the estimation of dosimetric quantities to ensure safety conditions to the patients and staff, the assessment of proper functioning of the equipment, and the evaluation of the quality of the produced images.

In Brazil, Ministry of Health Regulation $453^{32}$ is the document that regulates the commissioning and use of X-ray equipment in medical and dental radiologic imaging facilities. This regulation specifies which variables need to be measured and the frequency they should be done (annually, biannually, monthly or weekly). However, it does not specify measurement procedures for such tests, and neither has acceptance values for all measuring results. Quality control programs are usually based on recommendations from other national or international regulatory agencies to choose the procedures and acceptance intervals of each test. Examples of these agencies are IAEA (International Atomic Energy Agency) ${ }^{33}$, ANVISA (Agência Nacional de Vigilância Sanitária) ${ }^{34}$, and EURATOM (European Atomic Energy Community) ${ }^{35}$. They can also total or partially adopt technical documents of medical physics societies, such as AAPM $^{36}$ (American Association of Physicists in Medicine) or SEFM ${ }^{37}$ (Sociedad Española de Física Médica). These published procedures are then adapted to local characteristics that may exist in the particular clinical environment or available instrumentation.

Mostly these tests are performed acquiring images of an appropriate phantom and analyzing them. The following subsections provide details about CT phantoms (specially the Catphan500, used in the present work), how CT images are acquired and how the produced images must be handled.

\subsubsection{CT Phantoms}

Phantoms are simulating objects, containing known structures with known properties, designed to analyze medical equipment, testing its proper functioning and evaluating the quality of the produced images. In radiology modalities, there are also dosimetric 
phantoms, used to provide an estimation of the dose delivered to the patient or other quantities used to establish the compliance of the equipment to local regulations.

Conventional radiology uses a planar geometry for producing screen-film or digital projection images, and so its phantoms are plane-shaped. CT scanners collect data for imaging reconstruction adopting cylindrical geometry, and the phantoms for accessing its image quality or dosimetric properties have usually the same shape ${ }^{18}$. One such example is the AAPM phantom 610, developed in the seventies and used for over 20 years $\operatorname{after}^{38}$. This phantom has structures for evaluation of many quantities of interest for CT scanners quality control (such as spatial resolution, noise and slice thickness), and also has slots for inserting termoluminescent dosimeters. It had the inconvenience of needing to be filled with water. Nowadays, solid phantoms cast of water-equivalent materials are commercially available and water filled phantoms are not adopted in regular quality control or dosimetry in CT scanners.

\subsubsection{Catphan500 ${ }^{\circledR}$ Phantom}

The phantom used in the present work was the Catphan500 ${ }^{\circledR}$ (The Phantom Laboratories, Salem, USA), shown in Figure 1. It is constructed in 4 modules:

- CTP401: this module contains several structures for the assessment of a number of variables. There are 4 metallic pins for alignment check located in the surface of the phantom. There are 4 wire ramps with an inclination of $23^{\circ}$ relative to the $\mathrm{x}-\mathrm{y}$ plane for calculation of slice thickness and slice increment. There are 4 cylinders ( 3 of air and one of Teflon) for evaluation of pixel size, each cylinder located at a vertex of a square with $50 \mathrm{~mm}$ of side. There are 4 cylinders (of air, Teflon, LPDE and acrylic) for CT number calibration. Lastly, there are 5 acrylic spheres of different sizes used for low contrast resolution evaluation (these spheres are not used in this work). Figure 2 shows the schematics and a corresponding CT image of this module.

- CTP528: contains a set of bar patterns of different spatial frequencies for high contrast resolution evaluation, and 2 tungsten beads for Modulation Transfer Function $(M T F)$ estimation. The tungsten beads are slightly dislocated from each other in the z-axis, so they appear in different slices. Figure 3 shows the schematics and a corresponding CT image of this module. 
- CTP515: contains 3 sets of low contrast cylinders for evaluation of resolution at low contrast conditions. This module was not used in this work since the purpose of the CTP515 module is to assess the detectability of structures by human observers. Therefore, doing it with a software support would be inconsistent with the phantom design. Figure 4 shows the schematics and a corresponding CT image of this module.

- CTP486: this is a uniform section made of tissue-equivalent material, containing no structures, meant to estimate noise and Noise Power Spectrum (NPS). Figure 5 shows the schematics and a corresponding CT image of this module.
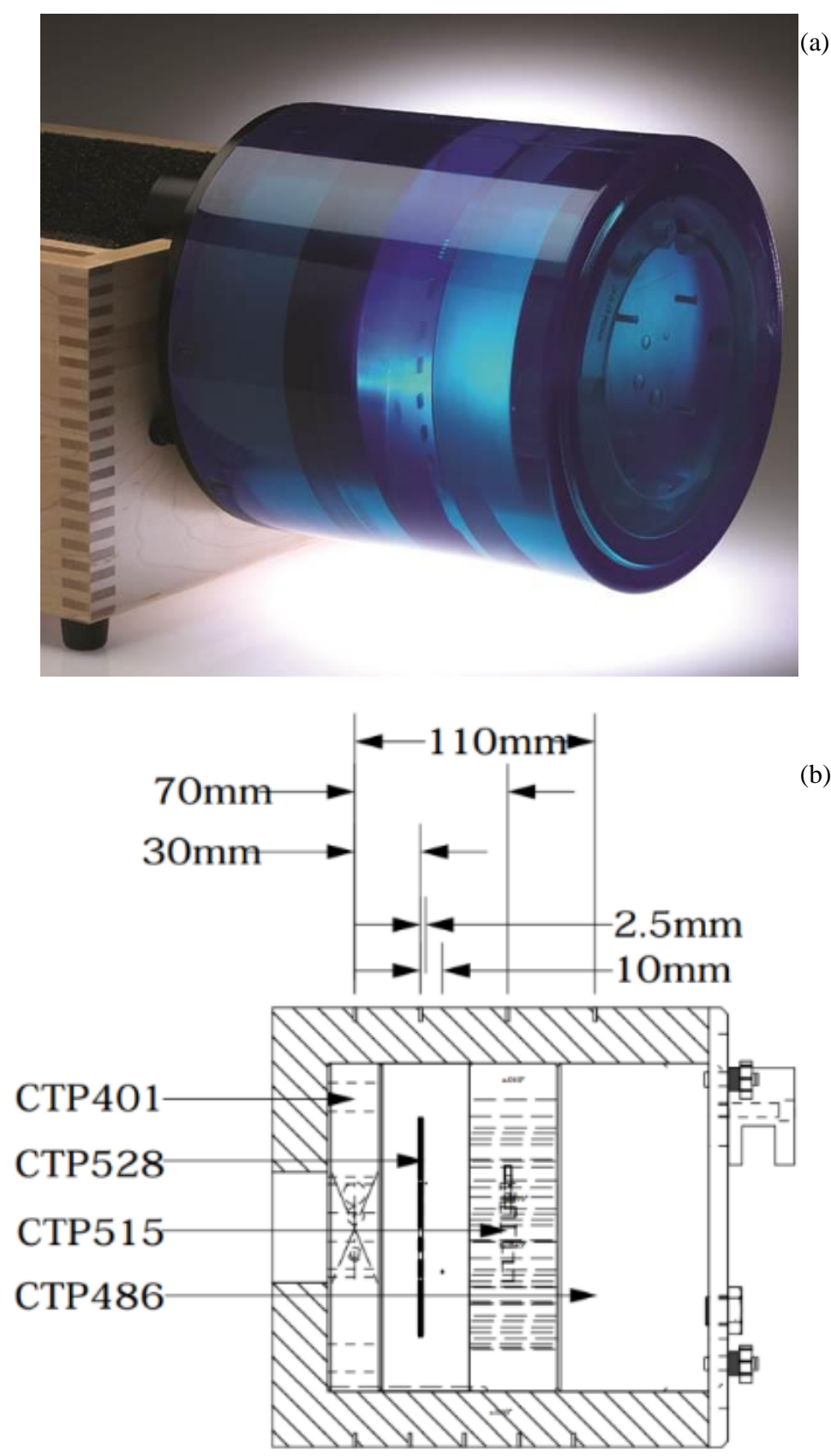
Figure 1: (a) photo of Catphan $500^{\circledR}$ and its case, used as counterweight (courtesy of Nersissian, D. Y.); (b) scheme of the internal structure of Catphan $500^{\circledR 39}$ (source: Catphan $500^{\circledR}$ and $600^{\circledR}$ Manual).

(a)

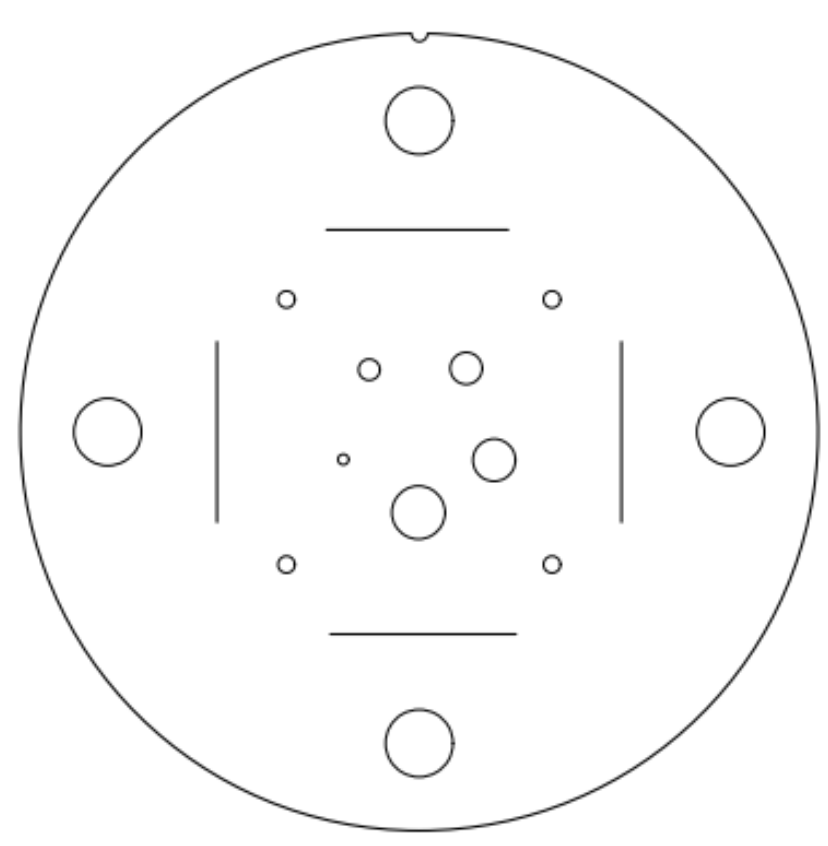

(b)

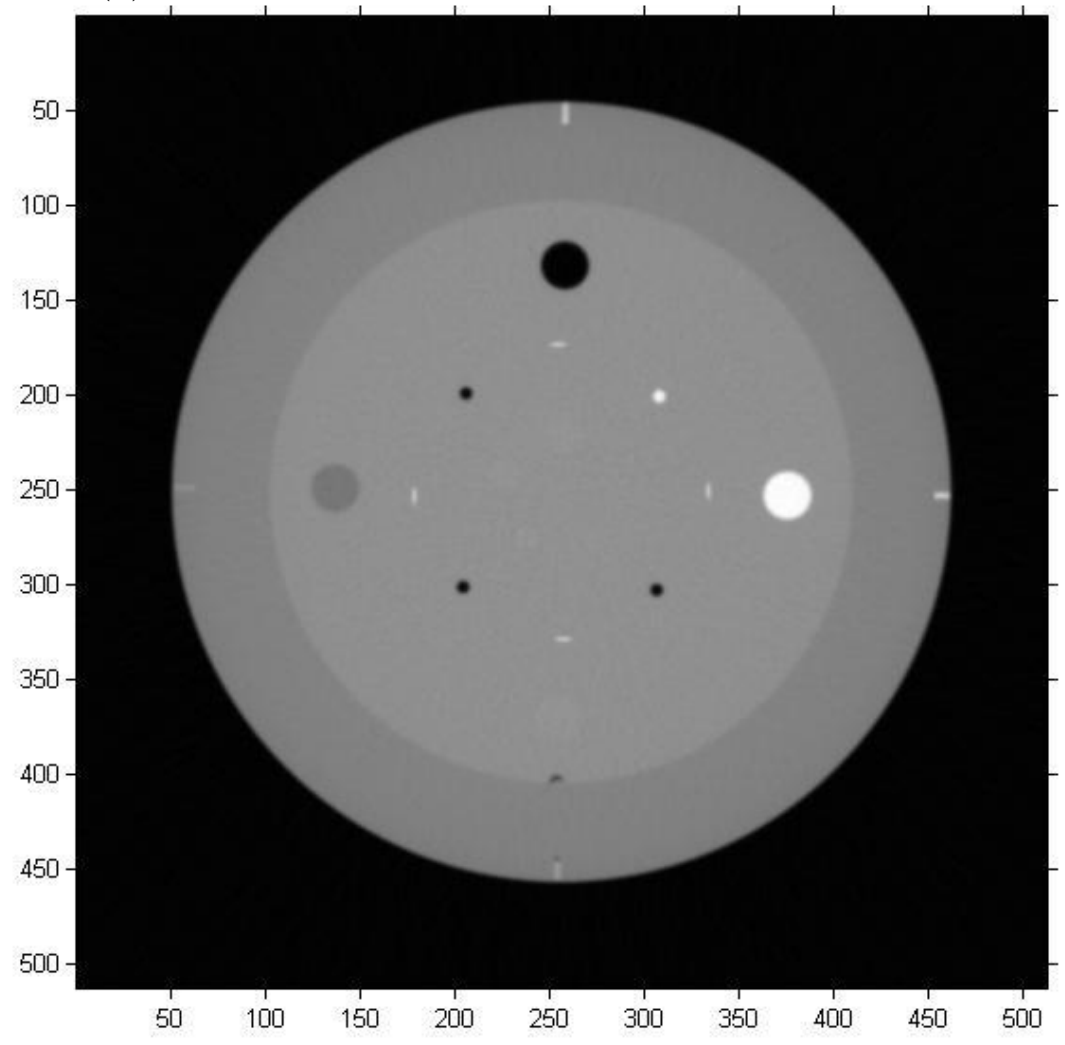

Figure 2: (a) scheme of the CTP401 module of Catphan $500^{\circledR}$ phantom (source: Catphan $500^{\circledR}$ and $600^{\circledR}$ Manual). (b) CT image of the central slice of the CTP401 module. 
(a)

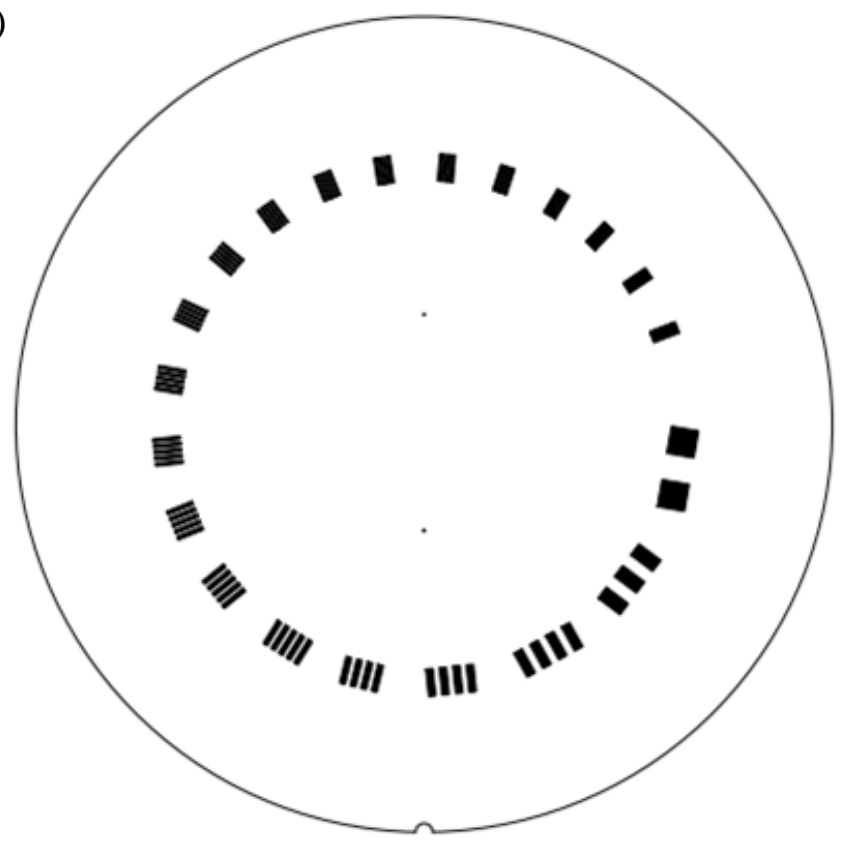

(b)

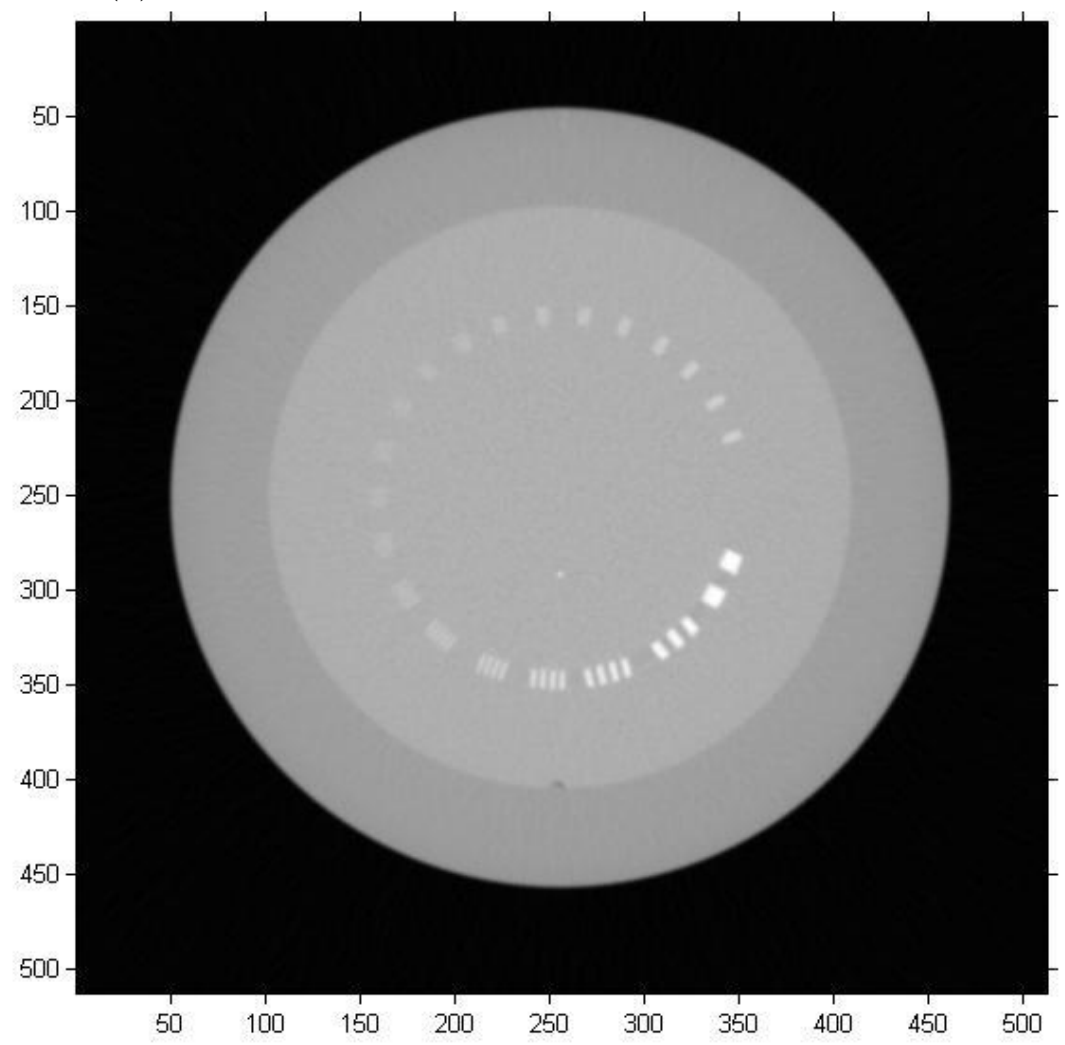

Figure 3: (a) scheme of the CTP528 module of Catphan $500^{\circledR}$ phantom (source: Catphan $500^{\circledR}$ and $600^{\circledR}$ Manual). (b) CT image of the central slice of the CTP528 module. The second tungsten bead does not appear in this CT image because it is slightly dislocated in the z-axis. 
(a)

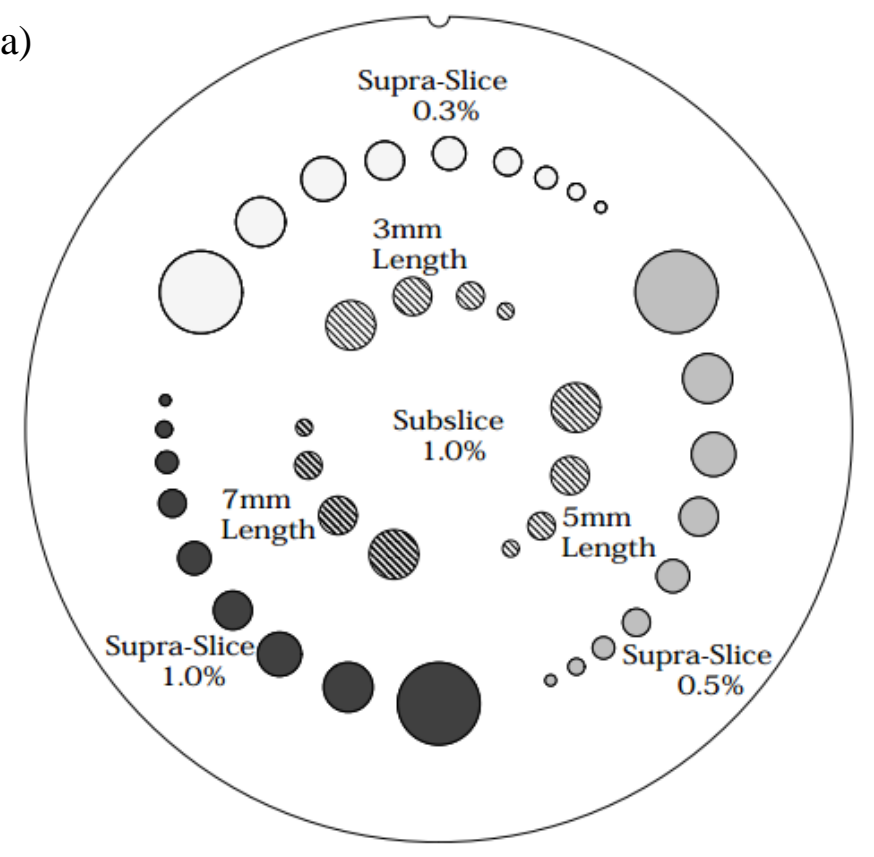

(b)

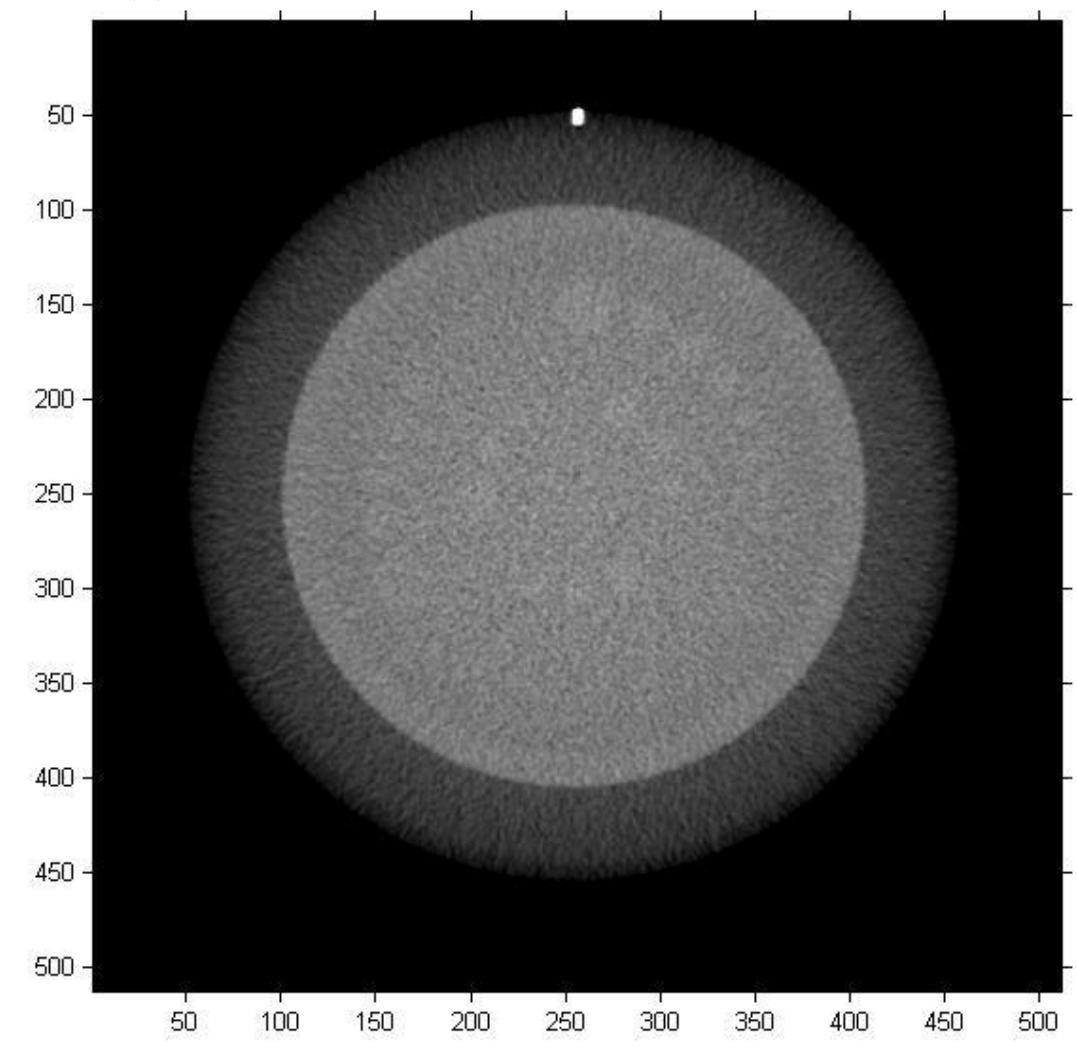

Figure 4: (a) scheme of the CTP515 module of Catphan $500^{\circledR}$ phantom (source: Catphan $500^{\circledR}$ and $600^{\circledR}$ Manual). (b) CT image of the central slice of the CTP515 module. The grayscale was adjusted to better visualize the structures, which have very low contrast. 
(a)

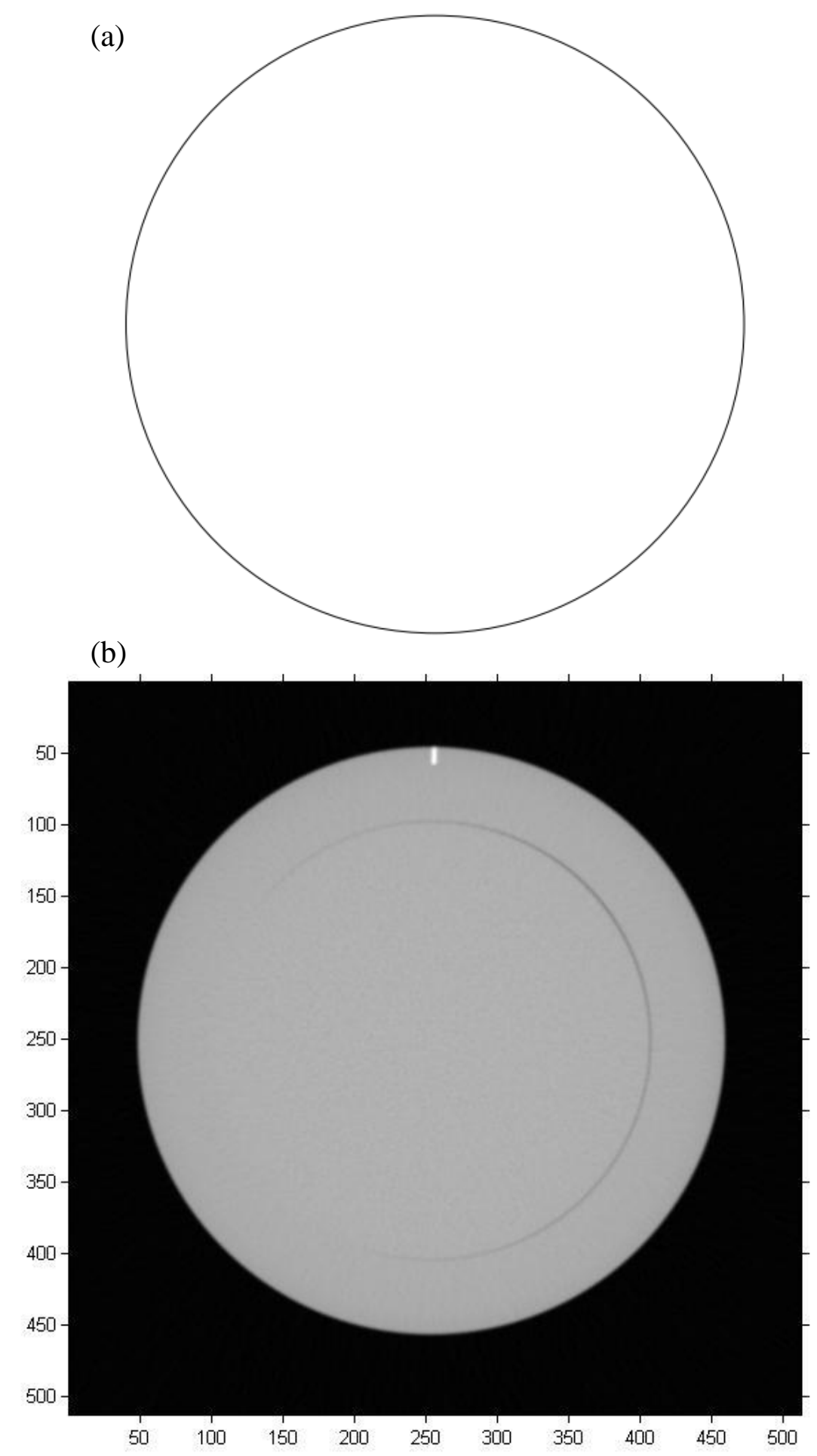

Figure 5: (a) scheme of the CTP486 module of Catphan $500^{\circledR}$ phantom (source: Catphan $500^{\circledR}$ and $600^{\circledR}$ Manual). (b) CT image of the central slice of the CTP486 module. 


\subsubsection{Image Acquisition}

To the image acquisition of an object (a patient, a phantom, etc) in a CT scanner, the first step is the positioning of the object on the examination table. All modern scanners have a laser alignment system for positioning the patient or phantom inside the gantry. CT phantoms such as the Catphan $500^{\circledR}$ also incorporate external fiducial marks for assisting the alignment of the device. The correct positioning of the phantom in the table is crucial for the analysis performed by the quality control professionals. Therefore, the adequate training and qualification of these professionals are essential criteria to the reliability of the results provided by the developed software. Figure 6 describes this process.

Once the object is positioned, the scanner is configured for the image acquisition. In this step, the following options ${ }^{40}$ (among others) are set:

- Scan mode: axial (the table moves and the X-ray tube rotate alternately) or helical (both X-ray tube and table move continually). In case the scan is in helical mode, the pitch is also selected (the pitch is defined as the table movement in one complete gantry rotation divided by the beam collimation ${ }^{41}$ );

- Slice Thickness: the smallest slice thickness is the length of the detector; multiple detectors can be summed, acquiring images of larger slice thickness. This choice will produce images with lower noise but reduced $\mathrm{z}$-axis spatial resolution $^{42}$;

- Scan Field of View (SFOV): a measurement of the size of the field that is irradiated by the X-ray beam ${ }^{43}$. The SFOV should be larger than the region of the patient to be imaged;

- Display Field of View (DFOV): a measure of the size of the irradiated field that is displayed in the image matrix ${ }^{43}$. It must be smaller or equal to the SFOV, and it directly determines the pixel size;

- X-ray tube voltage $(\mathrm{kVp})$ and anode current $(\mathrm{mA})$ : the X-ray tube voltage, associated to the filtration characteristics of the system, defines the radiation spectrum emitted by the equipment for producing the image. Both the spectrum and the anode current impacts directly on the patient dose and on the noise of the images $^{44}$. It must also be chosen whether or not to use the Automatic Exposure

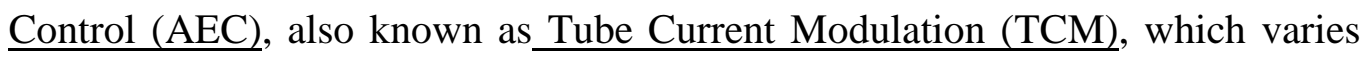


the current along the position according to the patient anatomy, intending to reduce the dose;

- $\quad$ Reconstruction Algorithm and Convolution Kernel: most scanners have a variety of reconstruction algorithms and convolution kernels available. These kernels can be adopted by the clinical protocol according to the required image quality characteristics. (see section 2.3).

Selecting particular values for these parameters defines a scan protocol. Diagnostic medical facilities usually have predefined clinical protocols (for example: a protocol called "head routine" used for most head scans).

Once every parameter is set, the scan can be performed. Usually, both the reconstructed images and the raw data are stored in a computer server. The raw data can be used again, if needed, to reconstruct new images with another reconstruction algorithm, kernel, DFOV, etc.

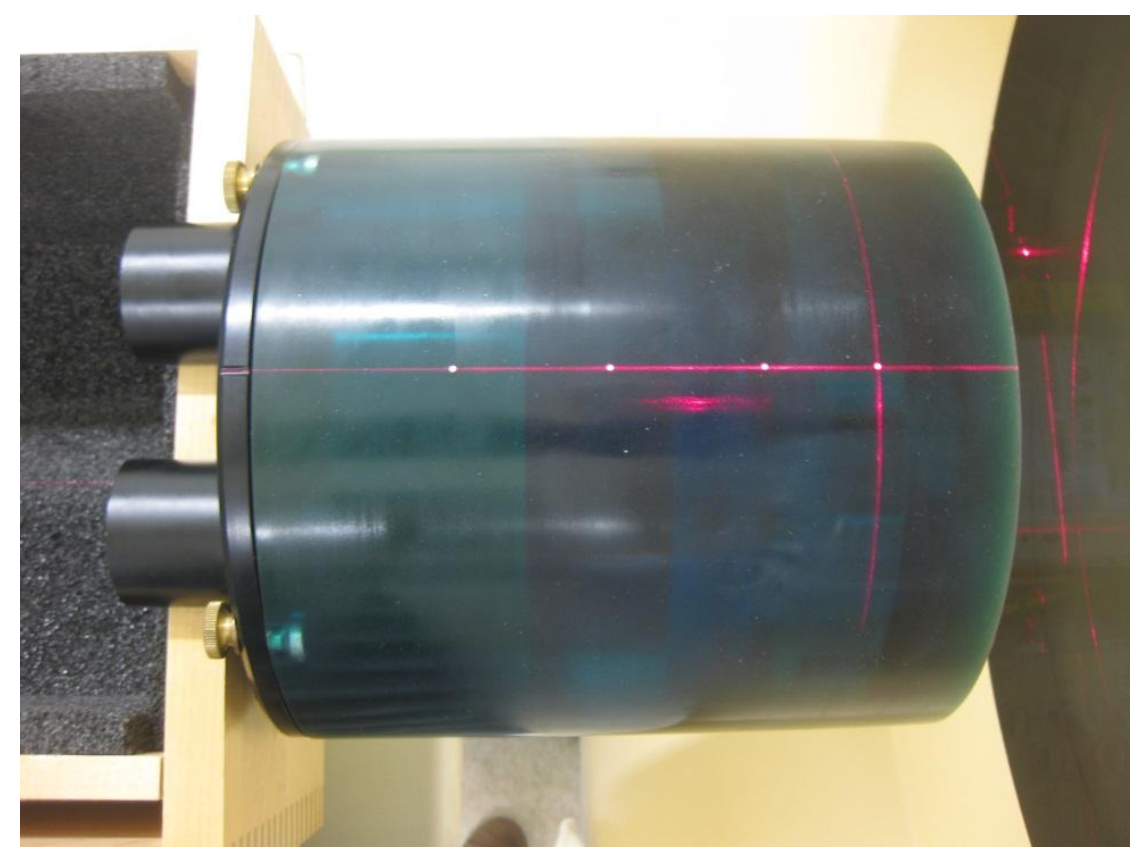

Figure 6: Catphan $500^{\circledR}$ in a CT scanner being aligned for an acquisition (courtesy of Nersissian, D. Y.).

\subsubsection{Medical Image Files}

The DICOM (Digital Imaging and Communications in Medicine) standard was created by the American College of Radiology (ACR) and the National Electrical Manufacturers Association (NEMA), with the goal of facilitating the treatment, storage 
and transfer of digital medical information ${ }^{45-47}$. The DICOM standard defines protocols for structuring the medical image files: the DICOM format (“.dcm” extension).

Users can find all the relevant information about the medical facility, the local network, the clinically relevant information about the patient to whom the image refers, the imaging modality employed, and all acquisition settings mentioned in section 2.1.3 for the case of CT images in the header of a DICOM file.

\subsection{Mathematical Description of Imaging Systems}

In a first approximation, imaging systems can be mathematically represented by a linear system $^{27}$, characterized by a linear operator $\hat{L}$ that, applied to an input signal $s_{i n}(\boldsymbol{r})$, produces an output signal $s_{\text {out }}(\boldsymbol{r})$ :

$$
\hat{L} s_{\text {in }}(\boldsymbol{r})=s_{\text {out }}(\boldsymbol{r})
$$

Where $\boldsymbol{r}$ is the coordinates vector $(x, y)$ or $(x, y, z)$ for a planar or volumetric image respectively.

In general, the output signal in a specific point is not directly related to the input signal at the same point (i.e., $s_{\text {out }}\left(\boldsymbol{r}_{\boldsymbol{i}}\right) \neq f\left[s_{\text {in }}\left(\boldsymbol{r}_{\boldsymbol{i}}\right)\right]$ ). Therefore, a point of the output can depend on many input points; equivalently, a single input point influences many output points. Formally, it means the operation $\hat{L} s_{i n}(\boldsymbol{r})$ can be written as the integral over the surface $\Omega$ :

$$
s_{\text {out }}(\boldsymbol{r})=\int_{\Omega} p\left(\boldsymbol{r}, \boldsymbol{r}^{\prime}\right) s_{\text {in }}\left(\boldsymbol{r}^{\prime}\right) d \boldsymbol{r}^{\prime}
$$

The function $p\left(\boldsymbol{r}, \boldsymbol{r}^{\prime}\right)$ is called impulse response ${ }^{27 ;}{ }^{48}$, and its complete evaluation determines how the imaging system produces its output from the input.

\subsubsection{Impulse Response and Point Spread Function (PSF)}

In the case where the input signal is a Dirac delta function, it follows that: 


$$
\begin{gathered}
s_{\text {in }}^{\delta}\left(\boldsymbol{r}^{\prime}\right)=\delta\left(\boldsymbol{r}^{\prime}-\boldsymbol{r}_{\mathbf{0}}\right) \Rightarrow s_{\text {out }}^{\delta}\left(\boldsymbol{r}, \boldsymbol{r}_{\mathbf{0}}\right)=\int_{\Omega} p\left(\boldsymbol{r}, \boldsymbol{r}^{\prime}\right) \delta\left(\boldsymbol{r}^{\prime}-\boldsymbol{r}_{\mathbf{0}}\right) d \boldsymbol{r}^{\prime}=p\left(\boldsymbol{r}, \boldsymbol{r}_{\mathbf{0}}\right) \\
P S F\left(\boldsymbol{r}, \boldsymbol{r}_{\mathbf{0}}\right) \equiv s_{\text {out }}^{\delta}\left(\boldsymbol{r}, \boldsymbol{r}_{\mathbf{0}}\right)=p\left(\boldsymbol{r}, \boldsymbol{r}_{\mathbf{0}}\right)
\end{gathered}
$$

The equation (3) is defined as the Point Spread Function $(P S F)^{27}$. This function represents the response (output) of the system for the case that the input signal is a Dirac delta function, and so it quantifies the impulse response $p\left(\boldsymbol{r}, \boldsymbol{r}_{\mathbf{0}}\right)$. For a photographic camera, for example, the $P S F$ is a picture of a very small and very intense light spot localized in ro. Measuring the PSF it is possible to the completely determine how the system produces the output from the input signal.

\subsubsection{Other Spread Functions}

Similarly as the PSF is defined as the system response to a punctual stimulus, other functions can be defined as the response to other kinds of input signals. The Line Spread Function $(L S F)^{49 ; 50 ; 51}$ is the response to a linear input signal (represented here as parallel to the $y$ axis):

$$
\begin{gathered}
s_{\text {in }}^{l}\left(x^{\prime}, y^{\prime}\right)=\delta\left(x^{\prime}-x_{0}\right) \Rightarrow s_{\text {out }}^{l}\left(x, y, x_{0}\right)=\int_{\Omega} P S F\left(x, y, x^{\prime}, y^{\prime}\right) \delta\left(x^{\prime}-x_{0}\right) d x^{\prime} d y^{\prime} \\
L S F\left(x, y, x_{0}\right) \equiv s_{\text {out }}^{l}\left(x, y, x_{0}\right)=\int_{-\infty}^{\infty} P S F\left(x, y, x_{0}, y^{\prime}\right) d y^{\prime}
\end{gathered}
$$

As shown in equation (4), the $L S F$ is the integral of the PSF over the $y$ ' axis.

The Edge Spread Function $(E S F)^{49 ; 52}$ is defined as the response to a border signal (a step function, or the Heaviside function, $\Theta$ ):

$$
\begin{gathered}
s_{\text {in }}^{\Theta}\left(x^{\prime}, y^{\prime}\right)=\Theta\left(x^{\prime}-x_{0}\right) \Rightarrow s_{\text {out }}^{\Theta}\left(x, y, x_{0}\right)=\int_{\Omega} \operatorname{PSF}\left(x, y, x^{\prime}, y^{\prime}\right) \Theta\left(x^{\prime}-x_{0}\right) d x^{\prime} d y^{\prime} \\
E S F\left(x, y, x_{0}\right) \equiv s_{\text {out }}^{\Theta}\left(x, y, x_{0}\right)
\end{gathered}
$$


Using the property that $\delta\left(x-x_{0}\right)=\frac{d}{d x} \Theta\left(x-x_{0}\right)$, the ESF relates to the LSF by a derivate:

$$
\operatorname{LSF}\left(x, y, x_{0}\right)=\frac{d}{d x} \operatorname{ESF}\left(x, y, x_{0}\right)
$$

\subsubsection{Translational Invariance}

A very important special case occurs when the system is invariant under translations, i.e., shifting the input signal shifts the output signal but does not change its shape. Such a system is often called a shift-invariant system. In this case:

$$
p\left(\boldsymbol{r}, \boldsymbol{r}_{\mathbf{0}}\right) \rightarrow p\left(\boldsymbol{r}-\boldsymbol{r}_{\mathbf{0}}\right)
$$

This particular form of impulse response ${ }^{53}$ implies:

$$
s_{\text {out }}(\boldsymbol{r})=\hat{L} s_{\text {in }}(\boldsymbol{r})=\int_{\Omega} P S F\left(\boldsymbol{r}-\boldsymbol{r}^{\prime}\right) s_{\text {in }}\left(\boldsymbol{r}^{\prime}\right) d \boldsymbol{r}^{\prime} \equiv P S F(\boldsymbol{r}) * s_{\text {in }}(\boldsymbol{r})
$$

Equation (8) shows that the operator $\hat{L}$ is actually the convolution product with the $P S F^{48}$ :

$$
\widehat{L}=P S F(\boldsymbol{r}) *
$$

\subsubsection{Convolution Properties}

By definition, the convolution has the same commutative, associative and distributive properties $^{54}$ of any integration operation:

$$
\begin{gathered}
f * g=g * f \\
f *(g * h)=(f * g) * h
\end{gathered}
$$




$$
f *(g+h)=f * g+f * h
$$

As shown through the $P S F$, the convolution of a function $f$ with a Dirac delta results in the same function $f$, i.e., the Dirac delta is the neutral element of the convolution:

$$
f * \delta=f
$$

Lastly, the convolution theorem ${ }^{55}$ states that the Fourier transform of a convolution is the simple product of the Fourier transform of each function involved:

$$
\mathcal{F}[f * g]=F . G
$$

\subsubsection{Modulation Transfer Function (MTF)}

In the previous sections, it was shown that specific inputs (the Dirac delta function, for example) offer useful insights of the properties of the imaging system (the PSF, in the same example). Applying this idea further, when the input signal has the form of a complex exponential of spatial frequency $\boldsymbol{f}=\boldsymbol{k} / 2 \pi$ :

$s_{i n}^{\boldsymbol{k}}(\boldsymbol{r})=e^{2 \pi i \boldsymbol{k} \cdot \boldsymbol{r}} \quad \Rightarrow \quad s_{o u t}^{\boldsymbol{k}}(\boldsymbol{r})=P S F(\boldsymbol{r}) * s_{i n}^{\boldsymbol{k}}(\boldsymbol{r})=\int_{\Omega} P S F\left(\boldsymbol{r}-\boldsymbol{r}^{\prime}\right) e^{2 \pi i \boldsymbol{k} \cdot \boldsymbol{r}^{\prime}} d^{2} r^{\prime}$

Applying the change of variables $\boldsymbol{r}^{\prime \prime}=\boldsymbol{r}-\boldsymbol{r}^{\prime}$ :

$$
\begin{gathered}
S_{\text {out }}^{\boldsymbol{k}}(\boldsymbol{r})=\int_{\Omega} P S F\left(\boldsymbol{r}^{\prime \prime}\right) e^{2 \pi i \boldsymbol{k} .\left(\boldsymbol{r}-\boldsymbol{r}^{\prime \prime}\right)} d^{2} r^{\prime \prime}=e^{2 \pi i \boldsymbol{k} \cdot \boldsymbol{r}} \int_{\Omega} P S F\left(\boldsymbol{r}^{\prime \prime}\right) e^{-2 \pi i \boldsymbol{k} \cdot \boldsymbol{r}^{\prime \prime}} d^{2} r^{\prime \prime} \\
S_{\text {out }}^{\boldsymbol{k}}(\boldsymbol{r})=s_{\text {in }}^{\boldsymbol{k}}(\boldsymbol{r}) . \mathcal{F}_{\boldsymbol{k}}[P S F(\boldsymbol{r})]=s_{\text {in }}^{\boldsymbol{k}}(\boldsymbol{r}) . P(\boldsymbol{f})
\end{gathered}
$$

$P(f)$ is the Fourier transform of the PSF to the variable $\boldsymbol{k}$, called System Transfer Function $^{27}$. $P(\boldsymbol{f})$ does not depend on $\boldsymbol{r}$. Therefore, for this specific input signal, the output has the same shape of the input, differing only by a multiplicative constant. However, although $P(\boldsymbol{f})$ is constant in $\boldsymbol{r}$, it is a function of $\boldsymbol{f}$, which means that each 
spatial frequency $\boldsymbol{f}$ is transmitted with unaltered shape, but with different relative amplitude. Therefore, the Modulation Transfer Function $(M T F)^{49 ; 52}$ is defined as:

$$
\operatorname{MTF}(\boldsymbol{f}) \equiv\left|\frac{P(\boldsymbol{f})}{P(0)}\right|
$$

The $M T F$ is the amplitude (relative to the zero frequency) that each component of the input is transferred to the output. The MTF is useful for imaging evaluating purposes since it is measurable $\mathrm{e}^{30 ; 56}$ and easily represented (it is a real function, in contrast to $P(f)$, which is complex function). However, the $M T F$ does not completely define $s_{\text {out }}$ from $s_{i n}$, it lacks the complex phase $\Phi(f)$.

The MTF corresponding to CT images will present radial symmetry due to the $\mathrm{x}-\mathrm{y}$ isotopy of both image acquisition hardware and image reconstruction algorithm used in modern equipment ${ }^{57-59}$. In the z-axis, the MTF of a CT scanner may present anisotropy due to the CT scanner geometry and the X-ray tube focal spot, but in the x-y plane this is much less expressive $\mathrm{e}^{60 ; 61}$. These assumptions will be taken into account on the MTF calculation methodology adopted in the present work and detailed on item 3.3.6.

\subsubsection{Frequency Space}

Since $P(f)$ provides an easy procedure to get $s_{\text {out }}$ from $s_{\text {in }}$ when $s_{\text {in }}$ is a complex exponential of frequency $f$, it may be useful to transform the generic input and output signals in functions of $\boldsymbol{f}$ instead of $\boldsymbol{r}$ (i.e., work in the frequency space instead of the position space). It can be done using the Fourier transform:

$$
S_{\text {in }}(\boldsymbol{f})=\mathcal{F}_{\boldsymbol{k}}\left[s_{\text {in }}(\boldsymbol{r})\right] \quad S_{\text {out }}(\boldsymbol{f})=\mathcal{F}_{\boldsymbol{k}}\left[s_{\text {out }}(\boldsymbol{r})\right]
$$

Applying the Fourier transform to both sides of Equation (8) and using the convolution theorem from equation (13):

$$
S_{\text {out }}(\boldsymbol{f})=\mathcal{F}_{\boldsymbol{k}}\left[P S F(\boldsymbol{r}) * S_{\text {in }}(\boldsymbol{r})\right]=P(\boldsymbol{f}) . S_{\text {in }}(\boldsymbol{f})
$$

A convolution product in the position space becomes a simple product in the frequency space, showing the computational advantage of working on the frequency domain. 


\subsubsection{Series of Imaging Systems}

Considering two linear systems described by their transfer functions $P_{1}(f)$ and $P_{2}(f)$, if the output of system 1 is the entrance signal of the system 2, then:

$$
\begin{gathered}
S_{\text {out }, 1}(\boldsymbol{f})=P_{1}(\boldsymbol{f}) \cdot S_{i n, 1}(\boldsymbol{f})=S_{i n, 2}(\boldsymbol{f}) \\
S_{\text {out }, 2}(\boldsymbol{f})=P_{2}(\boldsymbol{f}) \cdot S_{\text {in }, 2}(\boldsymbol{f})=P_{2}(\boldsymbol{f}) \cdot P_{1}(\boldsymbol{f}) \cdot S_{i n, 1}(\boldsymbol{f})
\end{gathered}
$$

So, it can be shown by induction that, by building a system from $N$ linear sub-systems in series, the output of the last as a function of the input of the first is:

$$
S_{\text {out }, N}(\boldsymbol{f})=S_{\text {in, } 1}(\boldsymbol{f}) \cdot \prod_{i} P_{i}(\boldsymbol{f})=S_{\text {in, } 1}(\boldsymbol{f}) \cdot P(\boldsymbol{f})
$$

Which means, the transfer function of the whole system $P(f)$ is the product of the transfer function of each part $P_{i}(f)^{27 ; 49}$. The same is true for the $M T F$ :

$$
\operatorname{MTF}(\boldsymbol{f})=\prod_{i} M T F_{i}(\boldsymbol{f})
$$

Equivalently, in the position space:

$$
P S F(\boldsymbol{r})=P S F_{1}(\boldsymbol{r}) * P S F_{2}(\boldsymbol{r}) * \ldots * P S F_{N}(\boldsymbol{r})
$$

\subsubsection{Noise and Noise Power Spectrum (NPS)}

Noise is the portion of a signal that carries no useful information ${ }^{62}$. It can or cannot have stochastic nature. The random variation of the pixel value on an image of a uniform object is an example of stochastic noise. This variation is mainly due to the discrete nature of the photons that produced the image. Non-stochastic noise (or structured noise) can be originated by equipment malfunction, incorrect image collection methodology, etc. 
Structured noise not necessarily has the random nature of stochastic noise, but it is also out of the user's control. This kind of noise can reduce the detectability of the relevant information in the image structure. Therefore, it is interesting to understand mathematically how filters act on noise, in order to choose the filter that adequately minimizes the loss of detectability caused by this intrinsic characteristic of detection systems.

Representing the noise by a random variable $\mathrm{x}(\boldsymbol{r})$, the simplest and most common way of estimating the noise of the imaging system is taking its standard deviation:

$$
\sigma=\sqrt{\left\langle(\mathrm{x}(\boldsymbol{r})-\langle\mathrm{x}(\boldsymbol{r})\rangle)^{2}\right\rangle}
$$

However, it can be more completely described by its Wiener spectrum ${ }^{63}$, or the Noise Power Spectrum (NPS), which quantifies the noise distribution over all spatial frequencies:

$$
N P S(\boldsymbol{f})=W_{\mathrm{x}}(\boldsymbol{f})=\lim _{\vartheta \rightarrow \infty}\left\langle\left|\widetilde{\mathcal{F}_{k}}[\mathrm{x}(\boldsymbol{r})]\right|^{2}\right\rangle=\mathcal{F}_{k}\left[R_{\mathrm{x}}(\boldsymbol{\rho})\right]
$$

Where $W_{\mathrm{x}}(\boldsymbol{f})$ is the Wiener spectrum, $\widetilde{\mathcal{F}_{k}}$ refers to the truncated Fourier transform ${ }^{63}$ of $\mathrm{x}(\boldsymbol{r})$ and $\vartheta$ is the truncation value. $R_{\mathrm{x}}(\boldsymbol{\rho})$ is the autocorrelation function ${ }^{64}$ of the random process $\mathrm{x}(\boldsymbol{r})$. These functions can be defined as:

$$
\begin{gathered}
\widetilde{\mathcal{F}_{k}}[\mathrm{x}(\boldsymbol{r})]=\int_{-\vartheta}^{\vartheta} \mathrm{x}(\boldsymbol{r}) e^{-2 \pi i k \boldsymbol{r}} d \boldsymbol{r} \\
R_{\mathrm{x}}(\boldsymbol{\rho})=\langle\mathrm{x}(\boldsymbol{r}) \mathrm{x}(\boldsymbol{r}+\boldsymbol{\rho})\rangle
\end{gathered}
$$

A system represented by a known transfer function, $P(f)$, will transforms the input noise $W_{\text {in }}(f)$ in the output noise $W_{\text {out }}(f)$ according to the relation:

$$
W_{\text {out }}(\boldsymbol{f})=|P(\boldsymbol{f})|^{2} W_{\text {in }}(\boldsymbol{f})
$$


The equation (24) shows that a system does not only modulate the input signal, it also modulates the noise. Then, knowing how the noise concentrates in particular range of frequency allows the use of specific filters in order to reduce it.

Appendixes $\mathrm{B}$ and $\mathrm{C}$ present a revision of probability theory and stochastic processes, and the derivation of equations (21) and (24).

\subsection{Computed Tomography Systems}

In this section, the mathematical formalism previously presented for generic imaging systems will be applied to CT systems. Although the model used is very simplified, it provides useful insights into the properties of CT scanners and the images they produce $^{27}$.

\subsubsection{Image Reconstruction}

Consider an object with a distribution of attenuation coefficients $s(\boldsymbol{r})$ being irradiated by an X-ray beam by one side, and a row of detectors measuring the transmitted radiation on the other side. The detector row is parallel to an axis $x^{\prime}$; the line from the tube to the center of the detector row defines the axis $y^{\prime}$; the system of coordinates $\boldsymbol{r}^{\prime}=\left(x^{\prime}, y^{\prime}\right)$ is rotated in relation to the coordinate system $\boldsymbol{r}=(x, y)$ by an angle $\theta$, but centered at the same point. Considering that the X-ray tube emits $\phi_{0}$ photons in the direction $y^{\prime}$ in a period of time $\Delta t$, the number of photons detected is:

$$
\phi_{\theta}\left(x^{\prime}\right)=\phi_{0} e^{-\int s\left(r^{\prime}\right) d y^{\prime}}
$$

For simplicity, the fact that the X-ray beam is not mono-energetic was ignored. Additionally, the fan shape of the beam and the fact that the detector row of a real CT system is assembled on a semi-arc structure were not considered. The letter $s$ was adopted for attenuation coefficients (instead of the classical $\mu^{65}$ ) to emphasize that these coefficients are, for the CT system, the signal to be computationally handled. Equation (25) can be linearized by taking the logarithm of both sides:

$$
\Psi_{\theta}\left(x^{\prime}\right) \equiv-\ln \left(\frac{\phi_{\theta}\left(x^{\prime}\right)}{\phi_{0}}\right)=\int s\left(x^{\prime}, y^{\prime}\right) d y^{\prime}
$$


The function $\psi_{\theta}\left(x^{\prime}\right)$ is called a projection of $s(\boldsymbol{r})$. Obtaining infinite projections at different angles $\theta(0 \leq \theta<\pi)$ it is possible to reconstruct the function $s(\boldsymbol{r})$, i.e., the image of the scanned object.

There are many algorithms for obtaining an image from its projections ${ }^{66-68}$. In the present work it will be discussed only the simplest and most popular algorithm, the filtered back-projection (FBP). The FBP algorithm is summarized in three steps:

- Back-projection: for each (one-dimensional) projection, create a twodimensional function $g_{\theta}\left(x^{\prime}, y^{\prime}\right)=\psi_{\theta}\left(x^{\prime}\right)$ (i.e., back-project $\psi_{\theta}\left(x^{\prime}\right)$ along the $y^{\prime}$ axis);

- $\quad$ Summation: sum all $g_{\theta}\left(x^{\prime}, y^{\prime}\right)$ functions to create the unfiltered image $\tilde{s}(\boldsymbol{r})$ :

$$
\tilde{s}(\boldsymbol{r})=\frac{1}{\pi} \int_{0}^{\pi} g_{\theta}\left(x^{\prime}, y^{\prime}\right) d \theta
$$

- Filtering: apply on $\tilde{s}(\boldsymbol{r})$ the (to be defined) filter that returns $\hat{s}(\boldsymbol{r})$, the best estimate of $s(\boldsymbol{r})$.

Writing the unfiltered output $\tilde{s}(\boldsymbol{r})$ explicitly as function of the input $s(\boldsymbol{r})$ :

$$
\tilde{s}(\boldsymbol{r})=\frac{1}{\pi} \int_{0}^{\pi}\left(\int_{-\infty}^{\infty} s\left(x^{\prime}, y^{\prime}\right) d y^{\prime}\right) d \theta
$$

This equation relates directly input to output of the system; so it is possible to estimate the $\widetilde{P S F}(\boldsymbol{r})$ of the system using the specific input $s^{\delta}\left(x^{\prime}, y^{\prime}\right)=\delta\left(x^{\prime}, y^{\prime}\right)$ :

$$
\begin{gathered}
\widetilde{P S F}(\boldsymbol{r})=\frac{1}{\pi} \int_{0}^{\pi}\left(\int_{-\infty}^{\infty} S^{\delta}\left(x^{\prime}, y^{\prime}\right) d y^{\prime}\right) d \theta \\
\widetilde{P S F}(\boldsymbol{r})=\frac{1}{\pi} \int_{0}^{\pi} \delta\left(x^{\prime}\right) d \theta
\end{gathered}
$$


Writing the function above in polar coordinates of the non-rotated referential and evaluating the resulting expression:

$$
\begin{gathered}
x^{\prime}=r \cos (\varphi-\theta) \\
\widetilde{P S F}(\boldsymbol{r})=\frac{1}{\pi r}
\end{gathered}
$$

Therefore, the unfiltered image of an object is:

$$
\tilde{s}(\boldsymbol{r})=\frac{1}{\pi r} * s(\boldsymbol{r})
$$

In the frequency space:

$$
\tilde{S}(\boldsymbol{f})=\tilde{P}(\boldsymbol{f}) \cdot S(\boldsymbol{f})=\frac{S(\boldsymbol{f})}{\pi k}
$$

Where $\tilde{P}(\boldsymbol{f})=\mathcal{F}_{\boldsymbol{k}}[\widetilde{P S F}(\boldsymbol{r})], \tilde{S}(\boldsymbol{f})=\mathcal{F}_{\boldsymbol{k}}[\tilde{S}(\boldsymbol{r})], S(\boldsymbol{f})=[s(\boldsymbol{r})]$ and $\boldsymbol{k}=2 \pi \boldsymbol{f}$.

Considering that this image has no noise, it would be possible to apply a filter $Q(\boldsymbol{f})=$ $\pi k$ so that:

$$
\hat{S}(\boldsymbol{f})=Q(\boldsymbol{f}) \tilde{S}(\boldsymbol{f})=S(\boldsymbol{f})
$$

However, in the presence of noise it is not advisable to add increasing "weights" to increasing frequencies ${ }^{69}$. Therefore, it is applied a filter $Q(\boldsymbol{f})=\pi k . J(\boldsymbol{f})$, where $J(\boldsymbol{f})$ is a two-dimensional window function appropriately chosen:

$$
\begin{gathered}
\hat{S}(\boldsymbol{f})=Q(\boldsymbol{f}) \tilde{S}(\boldsymbol{f})=S(\boldsymbol{f}) . J(\boldsymbol{f}) \\
\hat{S}(\boldsymbol{r})=q(\boldsymbol{r}) * \tilde{S}(\boldsymbol{r})=s(\boldsymbol{r}) * j(\boldsymbol{r})
\end{gathered}
$$


These equations show that, in a theoretical ideal CT system (ideal X-ray tube, ideal detectors, infinite projections, no noise, etc.), an arbitrarily high-quality image can be reconstructed. This function $J(f)$ is also the $M T F$ of the reconstruction algorithm, but does not represent the whole system. For the whole system, the MTF of other parts of the imaging system must be analyzed (the X-ray focal spot and detectors representing the most important factors).

The function $J(f)$ is often called the convolution kernel ${ }^{27}$. CT manufacturers provide a number of different kernels to be selected by the user according to the clinical needs. These provided kernels are supposed to be designed to be optimal for specific image characteristics, but normally these kernels are proprietary and the manufacturers do not reveal the exact functions incorporated into the algorithms.

\subsubsection{Noise Properties in CT systems}

The evaluation of noise properties of CT systems can be done analyzing the equation (26). In this equation, $\phi_{\theta}\left(x^{\prime}\right)$ refers to the photon count of a single detector at the given position and gantry angle during a specific period. To simplify the mathematical notation, the explicit $x$ ' dependency in $\phi_{\theta}\left(x^{\prime}\right)$ will be hereafter substituted by an index, $\phi_{\theta x}$. Defining the zero-mean variable $\Delta \phi_{\theta x}$ to simplify the following calculations:

$$
\Delta \phi_{\theta x} \equiv \phi_{\theta x}-\overline{\phi_{\theta x}}
$$

Supposing that the data detection in CT systems is a Poisson process ${ }^{70}$, it follows from equation B34 (appendix B) that the autocorrelation function of $\Delta \phi_{\theta x}$ is:

$$
\left\langle\Delta \phi_{\theta x} \Delta \phi_{\theta \prime x \prime}\right\rangle=\overline{\phi_{\theta x}} \delta_{\theta \theta}, \delta_{x x \prime}
$$

The projections $\psi_{\theta x}$ are defined in equation (26) as logarithms. Therefore, expanding them in the variable $\Delta \phi_{\theta x}$ around the zero:

$$
\Psi_{\theta x} \approx-\ln \left(\overline{\frac{\phi_{\theta x}}{\phi_{0}}}\right)-\frac{\Delta \phi_{\theta x}}{\overline{\phi_{\theta x}}}+\frac{\Delta \phi_{\theta x}^{2}}{2{\overline{\phi_{\theta x}}}^{2}}
$$


Calculating the mean of the projections $\overline{\psi_{\theta x}}$ and redefining the projections as zero-mean variables $^{71} \Delta \psi_{\theta x}$ :

$$
\begin{gathered}
\overline{\psi_{\theta x}}=\left\langle\psi_{\theta x}\right\rangle \approx-\ln \left(\overline{\frac{\phi_{\theta x}}{\phi_{0}}}\right)+\frac{1}{2 \overline{\phi_{\theta x}}} \\
\Delta \Psi_{\theta x} \equiv \psi_{\theta x}-\overline{\Psi_{\theta x}}=-\frac{\Delta \phi_{\theta x}}{\overline{\phi_{\theta x}}}+\frac{\Delta \phi_{\theta x}^{2}}{2{\overline{\phi_{\theta x}}}^{2}}-\frac{1}{2 \overline{\phi_{\theta x}}}
\end{gathered}
$$

And the autocorrelation of projections can then be calculated as:

$$
\left\langle\Delta \psi_{\theta x} \Delta \psi_{\theta \prime x \prime}\right\rangle=\frac{\delta_{\theta \theta} \delta_{x x \prime}}{\overline{\phi_{\theta x}}}
$$

This discrete result can also be evaluated using continuous variables:

$$
\begin{gathered}
\delta_{x x^{\prime}} \rightarrow \delta\left(x_{i}^{\prime}-x_{j}^{\prime}\right) \Delta x \\
\delta_{\theta \theta} \rightarrow \delta\left(\theta-\theta^{\prime}\right) \Delta \theta \\
\left\langle\Delta \Psi_{\theta}\left(x^{\prime}\right) \Delta \Psi_{\theta^{\prime}}\left(x^{\prime}+X^{\prime}\right)\right\rangle=\frac{\delta\left(X^{\prime}\right) \delta\left(\theta-\theta^{\prime}\right) \Delta x \Delta \theta}{\overline{\phi_{\theta}\left(x^{\prime}\right)}}
\end{gathered}
$$

Where $\Delta x$ is the length of the detector in the $x^{\prime}$ direction and $\Delta \theta$ represents the angular increment between projections.

The previously defined retroprojections $\mathrm{g}_{\theta}\left(x^{\prime}, y^{\prime}\right)$ are equal to $\psi_{\theta}\left(x^{\prime}\right)$ at any $y^{\prime}$, which means the autocorrelation of $\Delta g_{\theta}\left(x^{\prime}, y^{\prime}\right)$ have the same result of $\Delta \psi_{\theta}\left(x^{\prime}\right)$ :

$$
\left\langle\Delta \mathrm{g}_{\theta}\left(x^{\prime}, y^{\prime}\right) \Delta \mathrm{g}_{\theta^{\prime}}\left(x^{\prime}+X^{\prime}, y^{\prime}+Y^{\prime}\right)\right\rangle=\left\langle\Delta \Psi_{\theta}\left(x^{\prime}\right) \Delta \psi_{\theta^{\prime}}\left(x^{\prime}+X^{\prime}\right)\right\rangle
$$

The unfiltered image from equation (27) is (after transforming it in a zero-mean process): 


$$
\widetilde{\Delta s}(\boldsymbol{r})=\frac{1}{\pi} \int_{0}^{\pi} \Delta \mathrm{g}_{\theta}\left(x^{\prime}, y^{\prime}\right) d \theta
$$

Then, using (46) and (30), the autocorrelation of the unfiltered image is:

$$
\begin{gathered}
R_{\widetilde{\Delta s}}\left(\boldsymbol{r}, \boldsymbol{r}^{\prime}\right)=\left\langle\widetilde{\Delta \mathrm{s}}(\boldsymbol{r}) \widetilde{\Delta \mathrm{s}}\left(\boldsymbol{r}^{\prime}\right)\right\rangle \\
R_{\widetilde{\Delta s}}\left(\boldsymbol{r}, \boldsymbol{r}^{\prime}\right)=\frac{1}{\pi^{2}} \int_{0}^{\pi} d \theta \int_{0}^{\pi} d \theta^{\prime}\left\langle\left\langle\Delta \psi_{\theta}\left(x^{\prime}\right) \Delta \psi_{\theta^{\prime}}\left(x^{\prime}+X^{\prime}\right)\right\rangle\right\rangle \\
R_{\widetilde{\Delta s}}\left(\boldsymbol{r}, \boldsymbol{r}^{\prime}\right)=\frac{\Delta x \Delta \theta}{\pi^{2}} \int_{0}^{\pi} d \theta \frac{\delta\left(r \cos (\varphi-\theta)-r^{\prime} \cos \left(\varphi^{\prime}-\theta\right)\right)}{\overline{\phi_{\theta}}[r \cos (\varphi-\theta)]}
\end{gathered}
$$

Defining $\boldsymbol{L}=\left(L, \theta_{L}\right)=\boldsymbol{r}^{\prime}-\boldsymbol{r}$ and integrating the equation:

$$
R_{\widetilde{\Delta \mathrm{s}}}(\boldsymbol{r}, \boldsymbol{r}+\boldsymbol{L})=\frac{\Delta x \Delta \theta}{\pi^{2} L \overline{\phi_{\theta L+\pi / 2}}\left[r \cos \left(\theta-\theta_{L}\right)\right]}
$$

In the particular case when the scanned object is very large and approximately uniform:

$$
\overline{\phi_{\theta L+\pi / 2}}\left[r \cos \left(\theta-\theta_{L}\right)\right] \approx \text { const. }=\bar{\phi}
$$

and

$$
R_{\widetilde{\Delta s}}(\boldsymbol{r}, \boldsymbol{r}+\boldsymbol{L})=\frac{\Delta x \Delta \theta}{\pi^{2} \bar{\phi} L}
$$

Supposing the noise is stationary ${ }^{71}$ (i.e., it does not depend on the two specific positions $\boldsymbol{r}$ and $\boldsymbol{r}+\boldsymbol{L}$, only on the distance between them, $\boldsymbol{L}$ ), the NPS of the unfiltered image is then calculated with equation (21):

$$
W_{\widetilde{\Delta \mathrm{s}}}(\boldsymbol{f})=\mathcal{F}_{k}\left[R_{\widetilde{\Delta \mathrm{s}}}(\boldsymbol{r}, \boldsymbol{r}+\boldsymbol{L})\right]=\mathcal{F}_{k}\left[\frac{\Delta x \Delta \theta}{\pi^{2} \bar{\phi} L}\right]
$$




$$
W_{\widetilde{\Delta s}}(f)=\frac{\Delta x \Delta \theta}{2 \pi^{3} \bar{\phi} f}
$$

This NPS is then propagated to the filtered image $\widehat{\Delta s}(\boldsymbol{r})$ by equation (24), where $P(\boldsymbol{f})$ is the filter $Q(\boldsymbol{f})=\pi k . J(\boldsymbol{f})$ deduced in the previous section:

$$
\begin{array}{r}
W_{\widehat{\Delta s}}(\boldsymbol{f})=|P(\boldsymbol{f})|^{2} W_{\overparen{\Delta s}}(\boldsymbol{f}) \\
W_{\widehat{\Delta s}}(\boldsymbol{f})=\frac{2 f \Delta x \Delta \theta}{\pi \bar{\phi}}|J(\boldsymbol{f})|^{2}
\end{array}
$$

Similar result can be found in the literature ${ }^{72 ;}$. I3 . can be noted that the noise is concentrated at the low frequencies (the $f$ in the denominator) before filtering and it concentrates at high frequencies (the $f$ in the numerator) after filtering. It is also shown, using equation $\mathrm{C} 5$ at the appendix $\mathrm{C}$, that noise decreases with the inverse square root of the photon count density $\bar{\phi} / \Delta x$ :

$$
\sigma_{\widehat{\Delta s}}=\sqrt{\int_{\Omega} W_{\widehat{\Delta s}}(\boldsymbol{f}) d \boldsymbol{f}}=\sqrt{\frac{2 \Delta x \Delta \theta}{\pi \bar{\phi}} \int_{\Omega} f|J(\boldsymbol{f})|^{2} d \boldsymbol{f}}
$$

It is also worth remembering that, in all deductions in this and the previous sections, it was assumed that a CT system can be considered a linear shift-invariant system with stationary noise. It is known it is not true ${ }^{74}$. Nevertheless, shift-invariance and stationarity are good approximations, despite the possible systematic errors that may arise from these assumptions. 


\section{METHODS}

This chapter describes the basic design of the developed computer program, the algorithms associated to each part of the software and the chosen validation methods. In the last section, it also explains some of the software limitations and justifications for some design options chosen for the present development and the software validation.

\subsection{Software Specifications}

The software is intended to evaluate the following variables: pixel size, slice thickness, slice increment, CT number linearity evaluation coefficients, $M T F, N P S$, image noise and spatial resolution. It also automatically organizes the CT images in different protocols, sparing the user from the problem of searching through and evaluating hundreds of images unnecessarily.

\subsection{Software Structure}

The flowchart presented in Figure 7 describes the main blocks of the developed software. Each block is detailed in the appropriate following subsections.

Figure 8 show the software interface. The first step to use the software is selecting a folder containing the CT images to be analyzed. These images are typically not organized by any criteria and have counter-intuitive names, so the next step of the software is sorting them, using the button named "Sort" (section 3.2.1 details this process). After sorting the $\mathrm{CT}$ images, the software calculates the center of the phantom in each image (section 3.2.2 details this calculation) and identifies which images correspond to each Catphan section, separating them in the lists "CTP401", "MTF" and "noise". This separation can be done automatically by the button named "Identify Sections" (section 3.2.3 explains this process) or manually (the user can view each image on the folder and add them to the appropriate list). After the images are properly separated in lists, the buttons "CTP401", "MTF" and "Noise" can be used to calculate the variables of interest (sections 3.3.2 to 3.3.7 explain the algorithm for the calculations of each variable). Additionally, at any moment the user can click on an image of any list to display it, and also some important information from the DICOM header. The user may also start over, clicking the "RESET" button, which clears all lists (but does not 
undo the sorting process). Lastly, the software keeps a brief log of the performed operations.

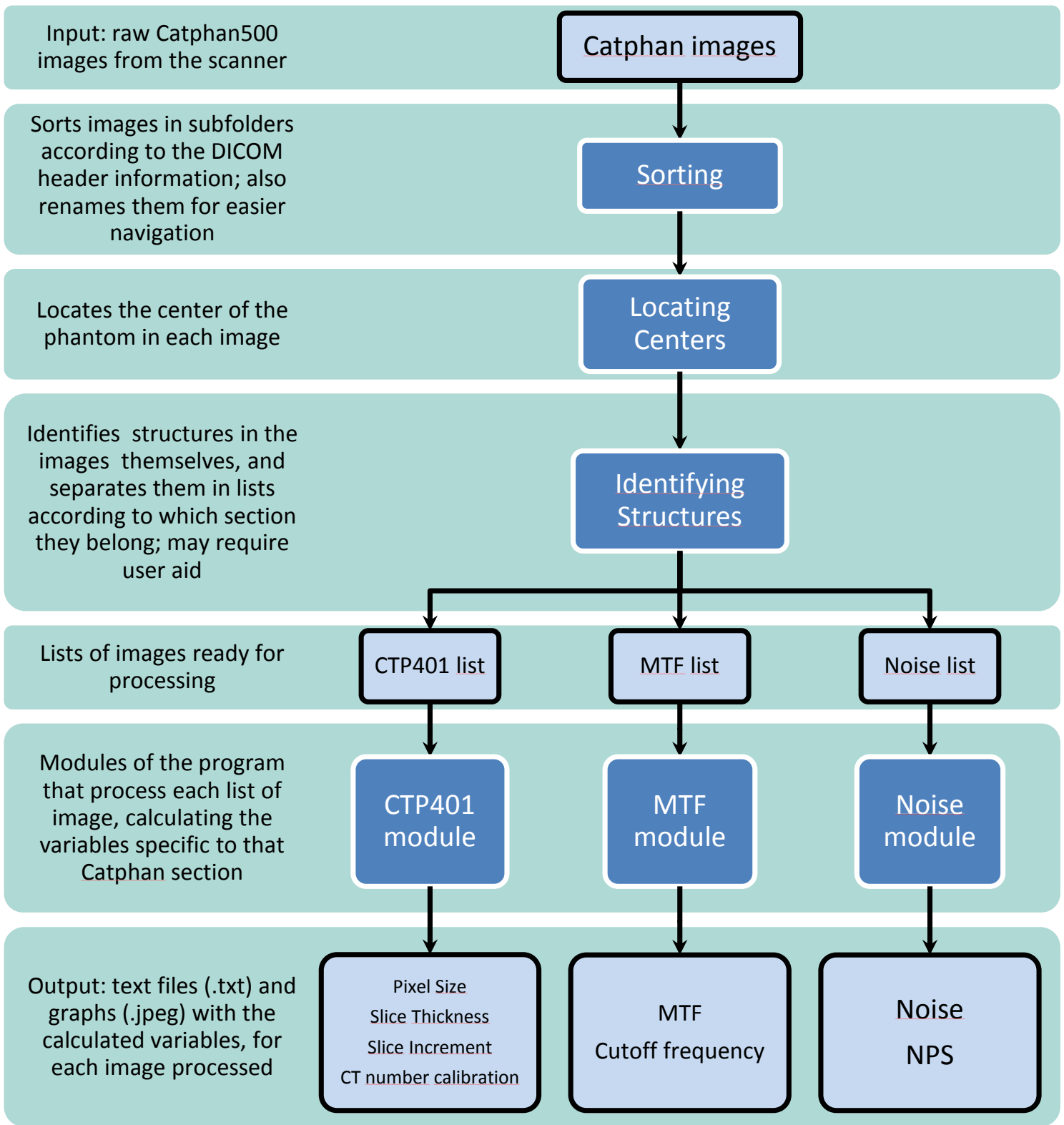

Figure 7: This flowchart summarizes how the software works. Initially, a folder containing the phantom images is provided by the user. The software then organizes the images in subfolders by acquisition protocol, and in a selected subfolder organizes them in lists by phantom section. Then, each list is used by its respective module to calculate their variables of interest. 

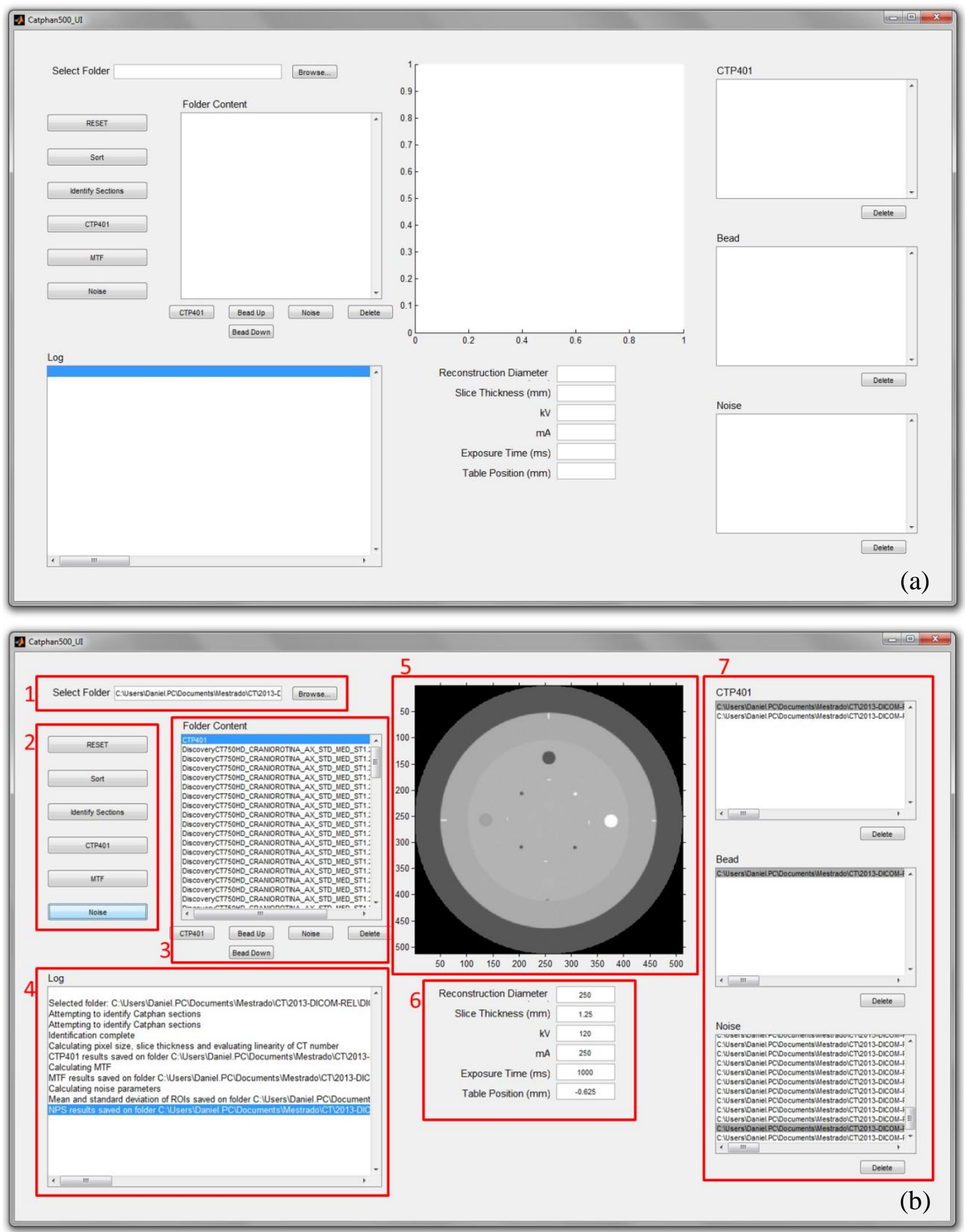

Figure 8: (a) Software interface, as it appears when the software is initialized. (b) Software interface at work, with its parts highlighted by the red boxes. 1) Box to select the folder containing the CT images to be analyzed. 2) The main commands of the software, used to manipulate the lists automatically and calculate the variables of interest. 3) List showing the images of the selected folder, with buttons to manually add images to the CTP401, MTF or Noise lists. 4) Log of operations previously performed. 5) Area for visualization of the CT images. 6) Information about the selected image extracted from the DICOM header. 7) Lists CTP401, MTF and Noise, with their respective Delete buttons. 


\subsubsection{Sorting Images from the Header Information}

Using the MatLab ${ }^{\circledR}$ (MathWorks, Natick, Massachusetts, USA) built-in tools to read DICOM files, it is possible to extract the following information from the header of the DICOM images:

- ManufacturerModelName: the model of the scanner (this field cannot be empty);

- SeriesDescription: a field filled by the user of the CT scanner with useful information about the acquisition protocol (if this field is empty, it is skipped by the software);

- ScanOptions: usually this is the field used to indicate if the acquisition was made in Axial or Helical mode; in some scanners this field is not available, and this information is located in other field of the DICOM header, but not easily identifiable. In such cases, this field is not used by the software);

- ConvolutionKernel: related to the reconstruction algorithm;

- FilterType: indicates the mathematical filter applied after image reconstruction;

- SliceThickness: a numeric parameter chosen by the scanner user that has significant impact on image quality.

For each combination of settings from these fields of the DICOM header (hereafter referred as protocol in the present document), the program creates a new folder in the hard disk, and moves all images taken according to that protocol into this new folder. Each image is also renamed as it is moved to the new folder in order to simplify the user's identification.

\subsubsection{Identification of the Center and Radius of the Phantom}

Once the images are classified by protocol, the software starts searching structures into the image matrices (not the headers). First, taking into account that the Catphan $500^{\circledR}$ phantom has cylindrical geometry, it determines the center and radius of the phantom in the image. This information is used by the software to conduct the automated identification of the structures present in an image. Without finding the center of the phantom the software cannot proceed and identify the internal phantom structures.

The sequence of operations carried out by the algorithm for each image $s_{i}(\boldsymbol{r})$ is described as following: 
- Take the gradient of the image, $\boldsymbol{g}_{\boldsymbol{i}}(\boldsymbol{r})=\boldsymbol{\nabla} s_{i}(\boldsymbol{r})$, and takes its absolute value $g_{i}(\boldsymbol{r})$, evidencing the border and the structures of the phantom present in the image;

- Suppose, initially, that the image of the phantom is exactly at the center of the image field of view. In a $512 \times 512$ pixels image, the center is at the position $\boldsymbol{c}_{0}=$ $(256,256)$, and the radius is initially supposed to be $R_{0}=210$ pixels. Limitations of this consideration in the case of misalignment of the phantom is described in section 3.4;

- Select 30 Regions of Interest (ROI's), with 31x31 pixels each, uniformly disposed around this initial circle on $g_{i}(\boldsymbol{r})$, which defines the image of the border of the phantom;

- In $g_{i}(\boldsymbol{r})$, any uniform region is valued as approximately zero, and any border has a very high positive value. The ROI's selected are dimensioned in order to only include the border of the phantom and its close vicinity, so it contains none of the phantom's internal structures. Therefore, inside one of these ROI's, the point of maxima is obligatorily on the border of the phantom. So, in each ROI, the software calculates the point of maximum pixel value and registers its position;

- The software fits all these points by a circle equation, finding the actual center $\boldsymbol{c}=\left(x_{c}, y_{c}\right)$ and radius $R$.

- The algorithm rounds the values found to $\boldsymbol{c}$ and $R$ to the nearest corresponding integers, because they will be used as matrix indexes in other parts of the software.

\subsubsection{Identifying Phantom Slices}

After the identification of the center and radius of the phantom, the software is able to identify the region of the image that corresponds to the alignment pins of Catphan $500^{\circledR}$. The section CTP401 of the phantom has 4 metal pins in the central slice (shown in Figure 9). So, the software selects 4 ROI's in the regions of the image where these pins must be located, and take the maximum inside these ROI's. In one ROI, if the resulting maximum is much higher than the background, the pin is in the ROI (the background is defined as the average pixel value in a region outside the selected ROI, towards the center of the phantom, as illustrated in Figure 9). If the maximum is close to the 
background, the pin is not inside the ROI. This procedure is executed for all images in the current folder. Once the software identifies an image containing 4 pins, it concludes that this image represents the CTP401 central slice. The position of this slice is obtained from the table position (i.e., the position of the slice along the $\mathrm{z}$-axis, the axis around which the X-ray tube and detectors rotate), which is read from the DICOM header. The distances from the CTP401 central slice to the central slice of the other sections of the phantom is known from the Catphan user's manual. In this manner, the software determines the position of the other phantom slices in the images sequence. Additional discussion regarding constrains and possible misalignment and inclination of the phantom during image acquisition is discussed at section 3.6 - Software Limitations.

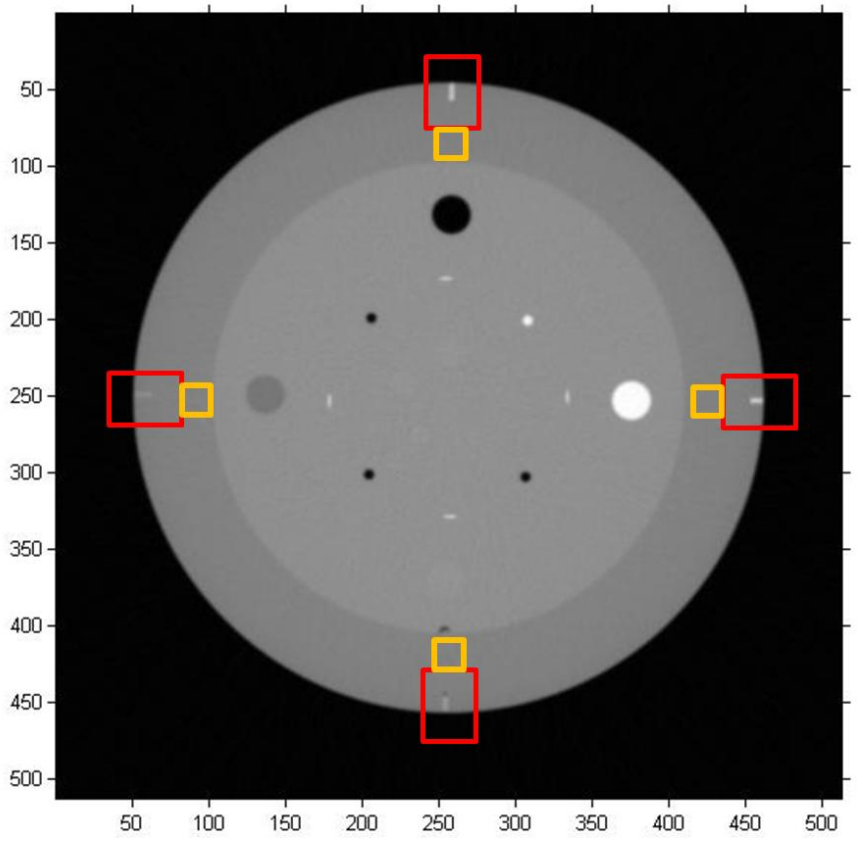

Figure 9: Image corresponding the Catphan $500^{\circledR}$ CTP401 central slice. The red boxes highlight the ROI's selected where the alignment pins are expected to be found. The yellow boxes highlight the regions used to calculate the background.

Once determined which images are from the CTP401 section, which images contain the tungsten beads and which contain the uniform section, the program separates these images in three lists ("CTP401", "MTF" and "noise", respectively). Since this sorting list can generate inconsistent image classification in the respective lists, a userdependent task must be performed, and consists on checking by the user if the images presented in the lists are really corresponding to the correct section of the phantom. For 
example, it is possible that the software selects an image which was acquired in a position in the phantom adjacent to the tungsten bead, but containing no structure and places it in the MTF list. In this case, this image should be removed before using this list to calculate the $M T F$. Otherwise, the calculation can result in inconsistent values. If such image classification occurs, the software user can remove them manually using "Delete" buttons incorporated in the user's interface, as shown in Figure 8.

\subsubsection{Modular Structure of the Software}

After the correct classification of the images in the lists labeled "CTP401", "MTF" and "noise", the software can proceed and calculate the variables of interest. These variables are divided in logical modules of the algorithm, according to the structures presented in the images allocated into each list. This procedure makes the software operation intuitive for a Catphan user, since this procedure corresponds to the traditional workflow for image quality control programs for CT machines. Additionally, the modular structure remains an open programming area to be used for creating additional modules by Matlab experienced users. Therefore, the software can be adapted to be used in similar phantoms, such as the Catphan models 600 or 700 .

Images from the CTP401 section of the phantom (allocated by the algorithm in the "CTP401" list in the computer memory) are used to calculate the following parameters: pixel size, slice thickness and slice increment. They are also used to perform the CT number calibration. Therefore, the algorithm calculates all these parameters in the logical module "CTP401". Refer to sections 3.3.2 to 3.3.5 for the detailed algorithm of each variable.

Similarly, images containing the tungsten bead (which were previously allocated in the "MTF" list) are used to calculate the MTF and to estimate the cutoff frequency from this function. Therefore, the software calculates these variables in the logical module "MTF". Lastly, images containing no structure (previously allocated in the list "noise") are used to calculate noise magnitude and NPS, so these variables are calculated in the logical module "noise". Refer to sections 3.3.6 and 3.3.7 for the detailed procedures for of the "MTF" and "noise" modules respectively.

\subsection{Algorithm of Measurement of each Variable}




\subsubsection{Locating Structures}

This section describes the general process of finding a particular structure on a given Catphan $500^{\circledR}$ image. This approach was adopted in the software for identifying different structures. Therefore, the procedure presented in this section can be generalized for any structure, and the specific details regarding specific structures are given in the corresponding sections.

A complete set of images was acquired, containing images of all Catphan sections, and this set was used as reference. This set was obtained in a GE Discovery CT750 HD machine, with FOV of $250 \mathrm{~mm}$, slice thickness of $1.25 \mathrm{~mm}$, in axial mode.

The center of the phantom in each of these images was obtained, $\boldsymbol{c}_{0}$, and the position of every structure was manually determined. If the center of a single structure is at the position $\boldsymbol{p}_{0}$, then its relative position to the center of the phantom is $\boldsymbol{p}_{\text {rel }}=\boldsymbol{p}_{0}-\boldsymbol{c}_{0}$. These values $\boldsymbol{p}_{0}$ and $\boldsymbol{c}_{0}$ were built into the software and they are adopted to find the structures of the phantom automatically without need of user's additional actions.

Using the values $\boldsymbol{p}_{0}$ and $\boldsymbol{c}_{0}$, the software determines the position of the same structure in another image. Initially, the software calculates the center of the phantom in this image, $\boldsymbol{c}$, by the procedure described in 3.2.2. Then, the software estimates the structure position, $\boldsymbol{p}$, adding the center $\boldsymbol{c}$ to the known relative position $\boldsymbol{p}_{\text {rel }}$ :

$$
\boldsymbol{p}=\boldsymbol{c}+\boldsymbol{p}_{\text {rel }}=\boldsymbol{c}+\boldsymbol{p}_{0}-\boldsymbol{c}_{0}
$$

The program then selects a 2D ROI around this point $\boldsymbol{p}$. The size and shape of this ROI vary according to the selected structure. For example, in the pixel size calculation (section 3.3.2) it is employed a square ROI of $11 \times 11$ pixels, whereas in the slice thickness calculation (section 3.3.3) it is employed a rectangular ROI of 97x17 pixels, oriented in the same direction as the ramp being observed. This ROI must be always large enough to contain the whole structure, but small enough to never overlap other structures. These ROI sizes were also incorporated in the software.

Inside a ROI containing one single feature, the center of the structure is determined by the point of minima (structure darker than the background) or maxima (structure lighter than the background). 


\subsubsection{Pixel Size}

Figure 10 shows an example of a CT image describing the central slice of the CTP401 module. For each image in the CTP401 list, the software calculates the pixel size $L_{1 \text { pix }}$ by the following procedure:

- Search for the positions of the air and Teflon cylinders by the algorithm described in 3.3.1, using a 11x11 pixels ROI (Figure 11);

- Locate the centers of the circles (Figure 12a), selecting the point of minima (air cylinders) or maxima (Teflon cylinder);

- Measures the distance between the centers of the circles, $d_{i j}=$ $\left\{d_{12,}, d_{23}, d_{34}, d_{41}\right\}$, in number of pixels (Figure 12b);

- As detailed in the item 2.1.2, the nominal distances between the center of each cylinder are $d_{\text {nominal }}=50 \mathrm{~mm}$, so the size of the side of a square pixel represented in the image is:

$$
L_{1 p i x}=\frac{50 m m}{\left\langle d_{i j}\right\rangle}
$$

Where $\left\langle d_{i j}\right\rangle$ is the average of all $d_{i j}$ measurements.

- The uncertainty of $L_{1 p i x}$ is then given by:

$$
\sigma_{L}=\frac{\sigma_{d} \cdot 50 \mathrm{~mm}}{2 .\left\langle d_{i j}\right\rangle^{2}}
$$

Where $\sigma_{d} / 2$ is the standard deviation of the mean of $d_{i j}$. In a properly functioning scanner, the measured pixel size is expected to be compatible with the nominal pixel size extracted from the DICOM header. However, there is no well-defined and widely adopted compatibility criterion for this task. 


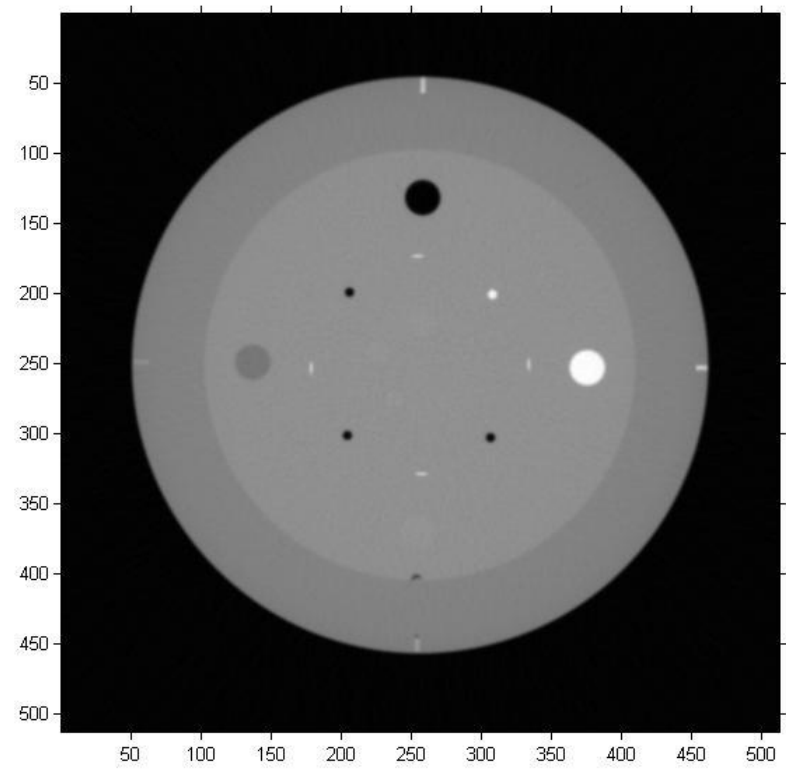

Figure 10: CT image showing the central slice of the module CTP401 of the Catphan $500^{\circledR}$ phantom. With this image, the software must locate the 4 cylinders of air and Teflon, calculate the distances between them, and use it to calculate the pixel size.

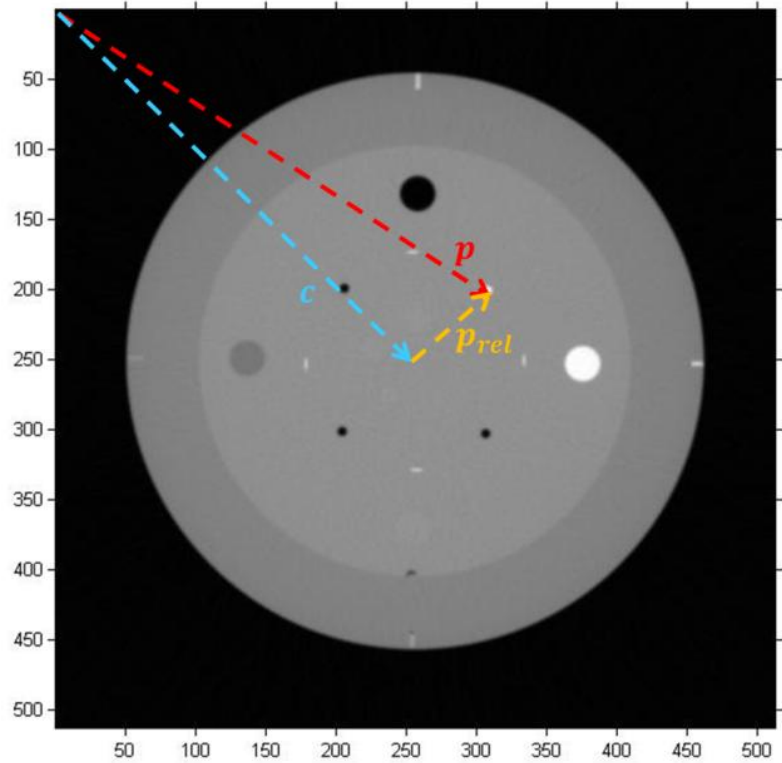

Figure 11: This picture illustrates the procedure to select a region containing the Teflon cylinder of Catphan $500^{\circledR}$. The software first calculates the center of the phantom $c$ (by the algorithm described in 3.2.2). Then it is added to the relative position of the cylinder $p_{\text {rel }}$ (built into the software, as explained in 3.3.1). This defines a point $p$, which is approximately where the cylinder is located. The software then selects a ROI around this point. 

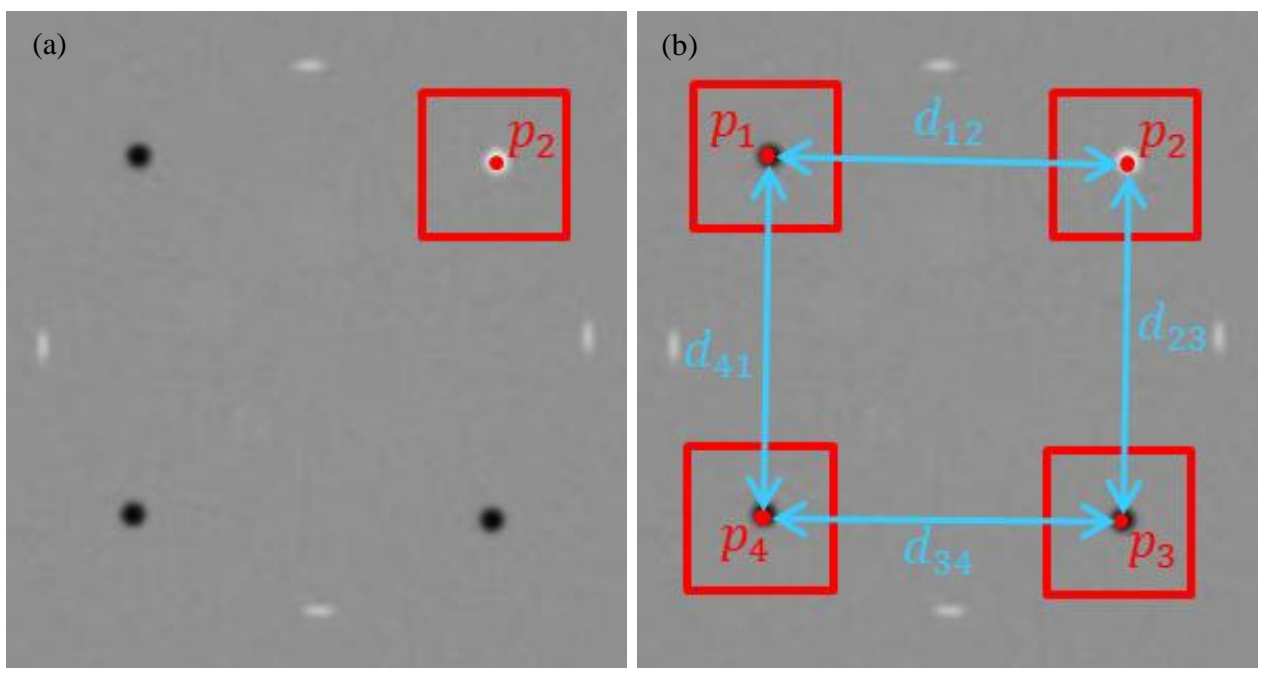

Figure 12: (a) CT image of the central part of the module CTP401 of the Catphan $500^{\circledR}$ phantom. The red square depicts the ROI previously defined containing the Teflon cylinder. The exact position corresponding to this cylinder in the image, $\boldsymbol{p}_{2}$, is then obtained by the point of maxima in this ROI (because the structure is lighter than the background; if it were darker it would be the point of minima). (b) The procedure depicted in Figure 11 and Figure 12a is repeated for the other 3 cylinders, obtaining $\boldsymbol{p}_{\mathbf{1}}, \boldsymbol{p}_{\mathbf{3}}$ and $\boldsymbol{p}_{\mathbf{4}}$. The software then measures the distances between each pair, illustrated in light blue.

\subsubsection{Slice Thickness}

The software calculates the slice thickness $\Delta z_{s t}$, for each image in the CTP401 list, using the following procedure:

- Define a ROI corresponding to the images of each of the 4 wire ramps described in 2.1.2, using the algorithm presented in section 3.3.1. It is used a ROI 99x 17 pixels ROI (oriented parallel to the ramp);

- Find the point of maxima of these ROI's (this is illustrated in Figure 13a);

- For each ramp $j$, the software:

Traces a profile parallel to the ramp, crossing its maxima (Figure 13b);

Subtracts the background from the profile. This background was taken as the mean pixel value in a $7 \times 7$ pixels square ROI displaced 7 pixels from the point of maxima of the ramp in the direction perpendicular to the ramp;

Finds the maximum of the profile $\left(h_{m}\right)$ and calculates the full width at half maximum $(F W H M)$ of the peak in the profile;

$>$ As mentioned in the item 2.1.2, the ramp has inclination of $23^{\circ}$ relative to the $x-y$ plane. Therefore, the slice thickness estimated only with the $j$-th ramp is: 


$$
\Delta z_{s t, j}=F W H M \cdot \tan \left(23^{\circ}\right) \cdot L_{1 p i x}
$$

- The slice thickness is then the mean of the thickness measured with each of the four ramps:

$$
\Delta z_{s t}=\left\langle\Delta z_{s t, j}\right\rangle_{j}
$$

- The uncertainty is then the standard deviation of the mean, plus an uncertainty derived from the image noise, estimated as $2 \%$ of the mean slice thickness obtained $\Delta z_{s t}$ (the determination of this value is described in Appendix D).

The obtained slice thickness should be compatible with the slice thickness selected by the user at the moment of the acquisition. Currently, the conformance criterion ${ }^{34}$ for slice thickness is:

- If the nominal slice thickness $\Delta z_{s t, n o m}$ is lower than $2 \mathrm{~mm}$, the obtained slice thickness is acceptable if $\left|\Delta z_{s t, n o m}-\Delta z_{s t}\right|<\Delta z_{s t, n o m} / 2$;

- If $\Delta z_{s t, n o m}$ is larger than $2 \mathrm{~mm}$, the obtained slice thickness is acceptable if $\left|\Delta z_{\text {st,nom }}-\Delta z_{\text {st }}\right|<1 \mathrm{~mm}$. 

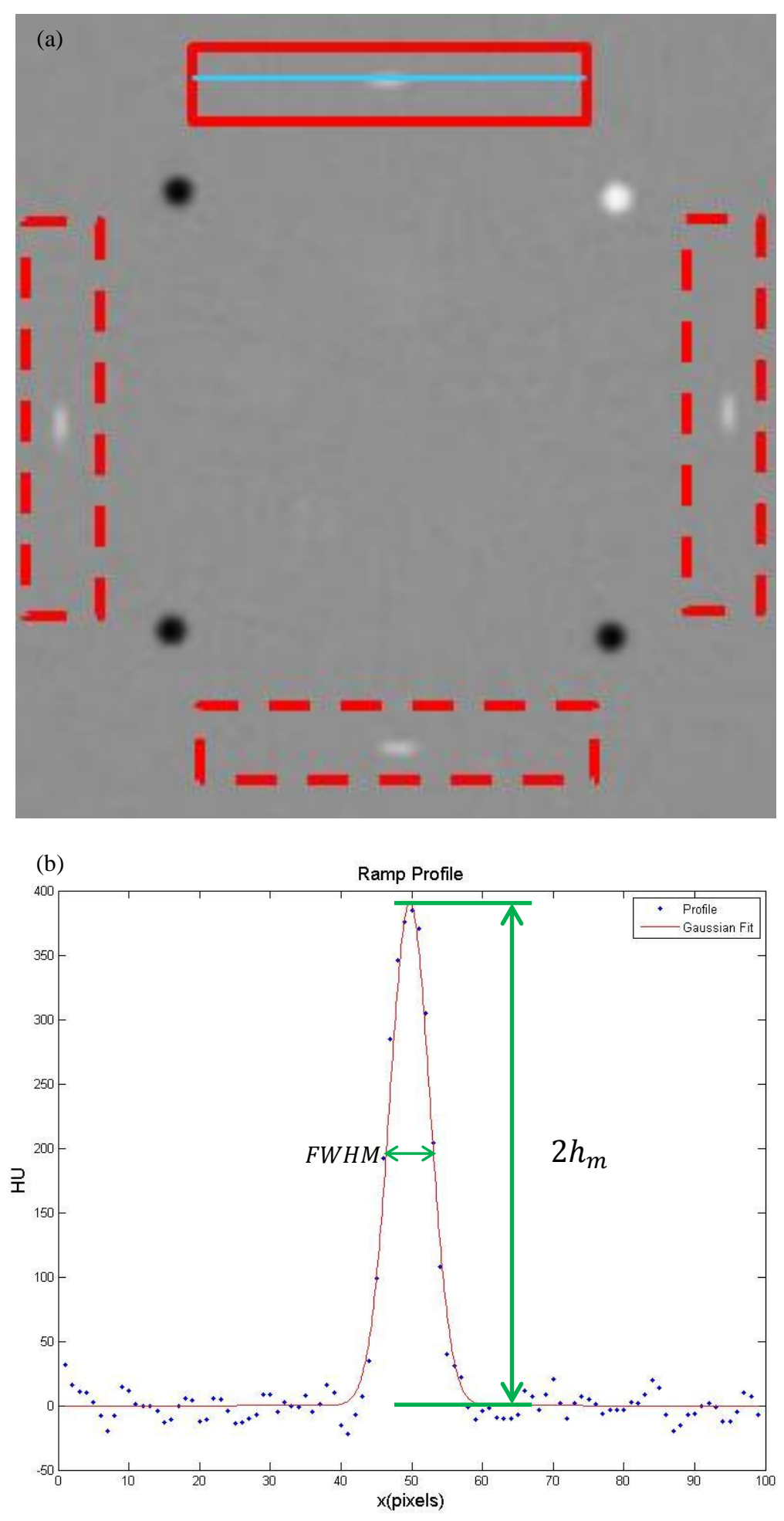

Figure 13: (a) CT image of the central part of the module CTP401 of the Catphan phantom. The procedure to define a ROI containing a ramp (solid red rectangle) is analogous to the procedure depicted in Figure 11 and Figure 12. A profile is then traced parallel to the ramp, crossing its point of maxima (light blue line). This procedure is repeated for each of the 4 ramps (dashed red rectangles). (b) Profile of the ramp shown in the solid red square of Figure 13a, subtracted of the background. The red line is a Gaussian function fitted to the profile. The green lines indicate the total height of the peak $\left(2 h_{m}\right)$ and the full width at half maxima $(F W H M)$. The FWHM is used to calculate the slice thickness. 


\subsubsection{Slice Increment}

The slice increment $\Delta z_{i n c}$ calculation uses most of the same algorithm used for slice thickness calculation. However, at the step where the FWHM of the peak would be calculated, the position of the peak $p_{1}$ is calculated instead. This procedure is applied to the central CTP401 slice (the slice depicting the four alignment pins) and also to the next slice (where the ramps appear slightly dislocated, as shown in Figure 14), obtaining $p_{2}$ from the second. With the difference $\Delta x=\left|p_{1}-p_{2}\right|$, shown in Figure 15, the slice increment is calculated:

$$
\Delta z_{\text {inc,j }}=\Delta x \cdot \tan \left(23^{\circ}\right) \cdot L_{1 p i x}
$$

The final slice increment is calculated taking the average of the values obtained for each ramp, and the uncertainty is the standard deviation of the mean. This value is to be compared with the difference between the table position of both images used, i.e., the nominal slice increment. 

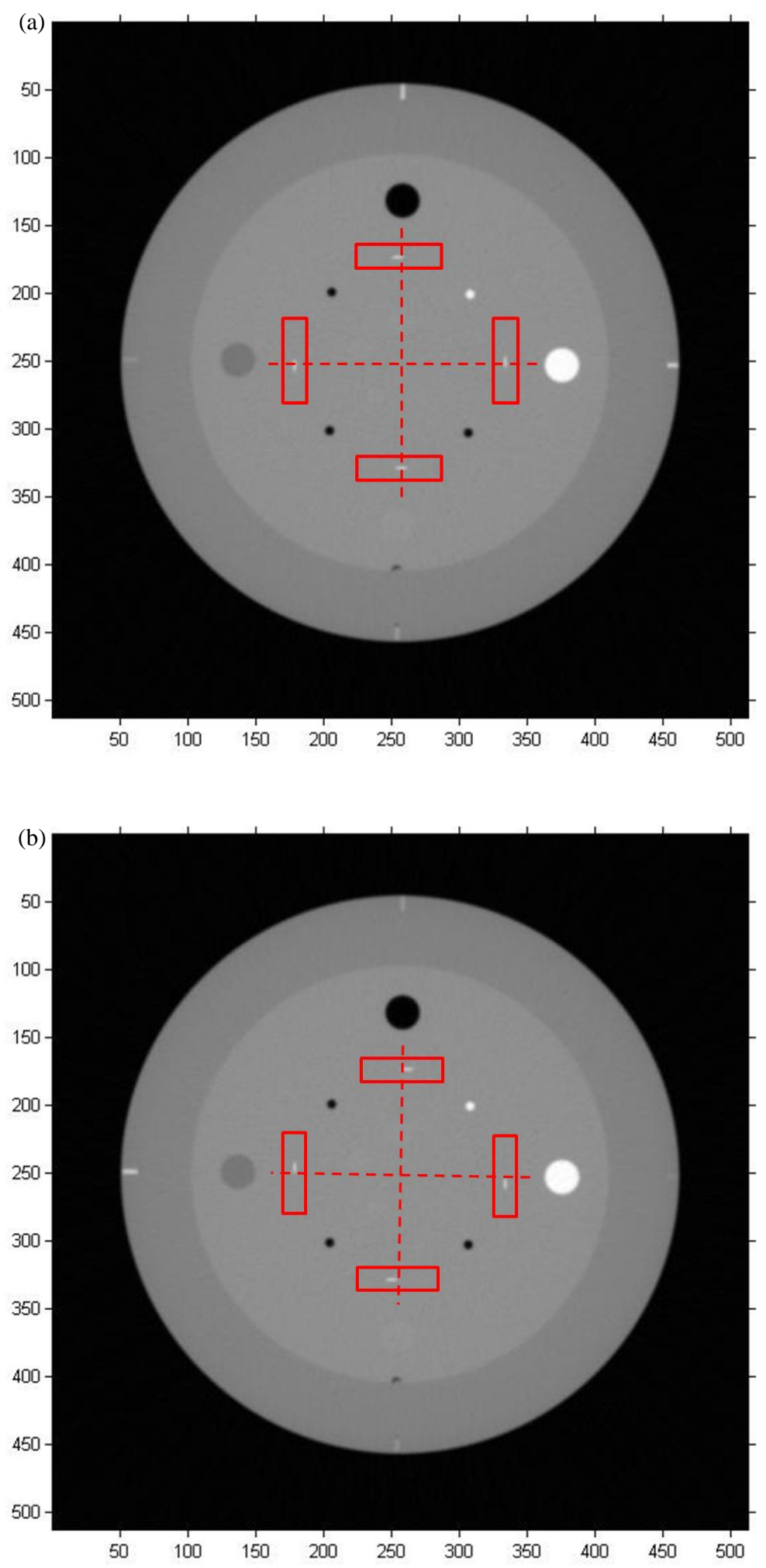

Figure 14: (a) CT image of the central slice of the CTP401 module of Catphan500 ${ }^{\circledR}$. (b) CT image of another slice of the CTP401 module of Catphan500 ${ }^{\circledR}$. This is not the central slice of the module: notice the clockwise dislocation of the 4 ramps (highlighted in the red boxes) when compared to (a). 


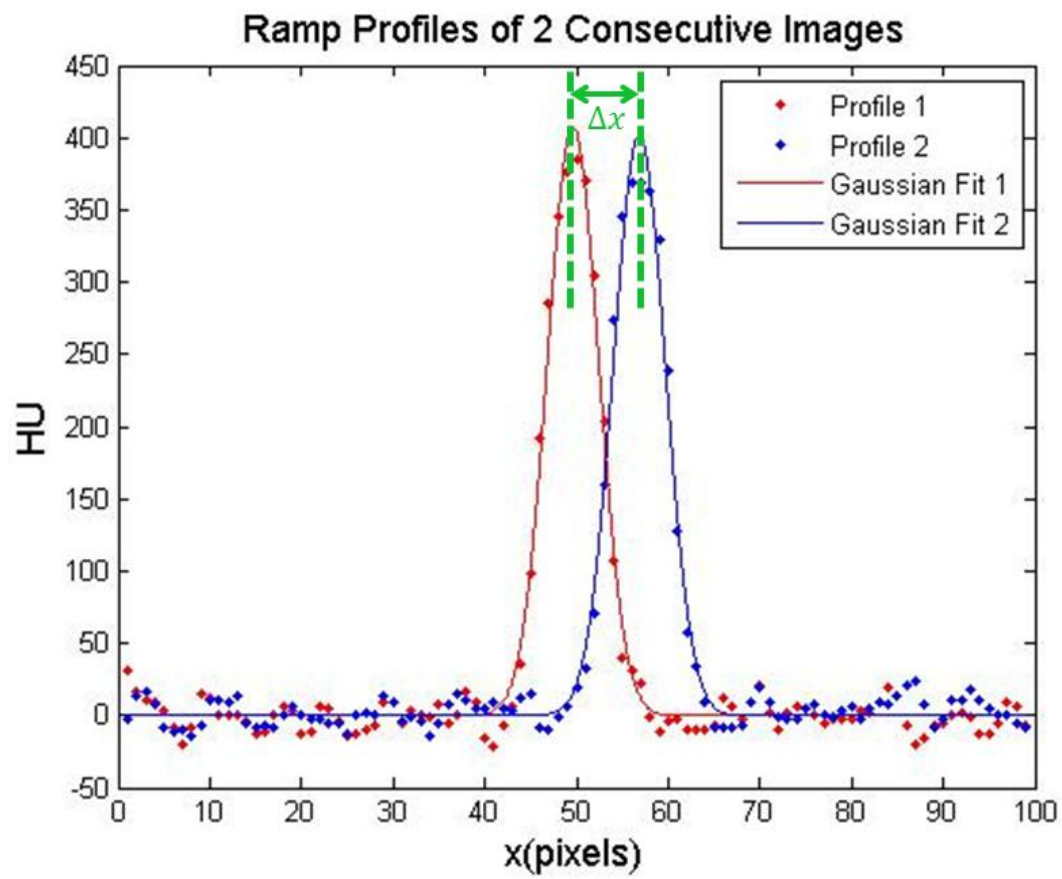

Figure 15: ramp profiles of 2 consecutive images of CTP401 module, with the center of each peak highlighted by the dashed line and the distance between them represented by $\Delta x$.

\subsubsection{CT Number Calibration}

The software evaluates the linearity of the CT number, for each image in the CTP401 list, by the following procedure:

- $\quad$ By the procedure described in 3.3.1 (ROI size 7x7 pixels), define a ROI inside each of the 4 cylinders of different materials (air, Teflon, LPDE and acrylic) shown in Figure 16. As each cylinder is univocally identified by the phantom design, the material of these cylinders are identified by the ROI position;

- Calculate the mean and mean standard deviation of the pixel value inside each ROI (obtaining $\overline{C T}$ and $\sigma_{C T}$ for each material);

- Fit (with linear least squares method) the measured CT number $(\overline{C T})$ to the expected CT number of each ROI $\left(C T_{\text {exp }}\right)$ given by the phantom user's manual $\left(\overline{C T} \approx a+b \cdot C T_{\text {exp }}\right)$. The fitting process uses a built-in linear least squares tool incorporated in MatLab ${ }^{\circledR}$;

- Save the coefficients $a$ and $b$, and the $R^{2}$ coefficient resulting from the fit, in a text file. 
If the CT number calibration is adequate, the coefficients $a$ and $b$ should be close to 0 and 1 respectively, so that $\overline{C T} \approx C T_{\text {exp }}$. There is no widely accepted compatibility criterion for this task.

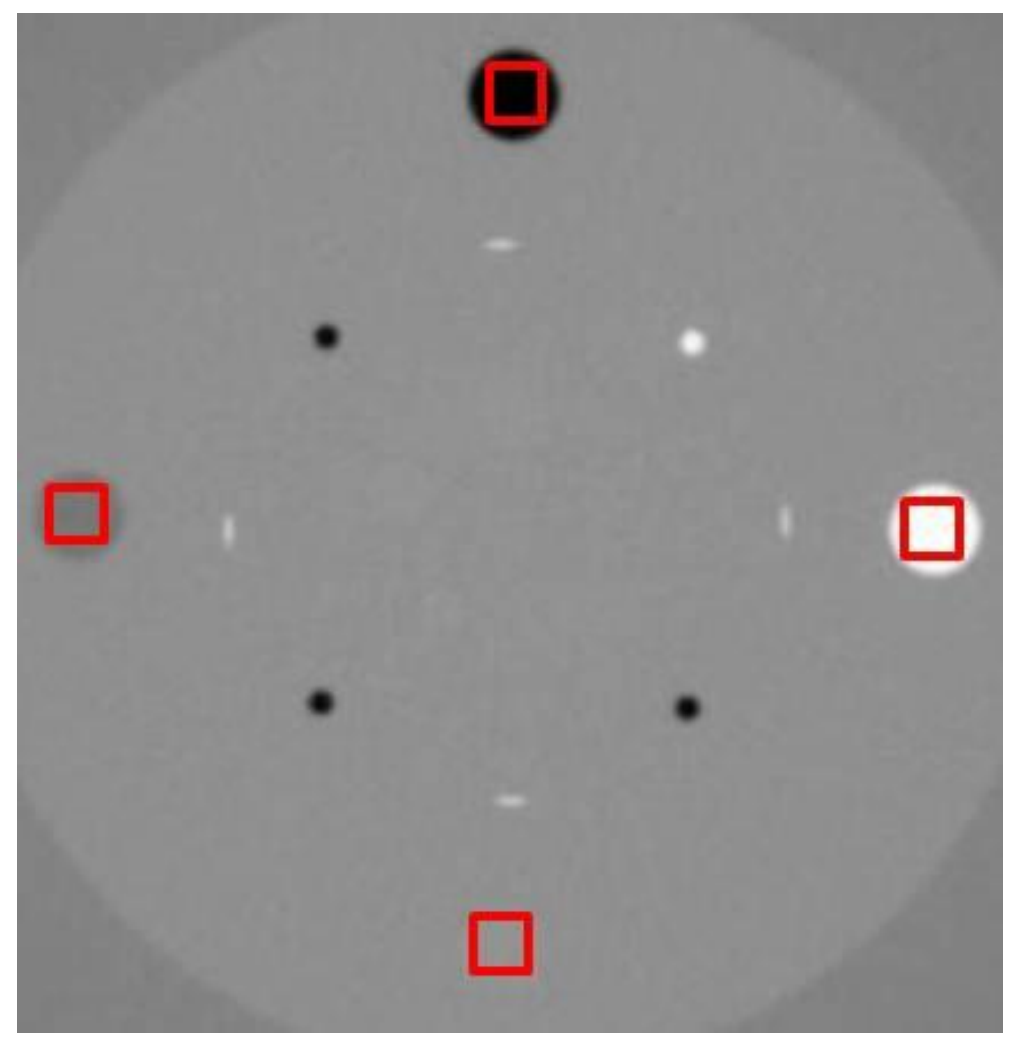

Figure 16: CT image of the central part of the module CTP401 of the Catphan phantom; the red boxes highlight the 4 cylinders used in the calibration of the CT number. Clockwise from the top, the material of each cylinder is: air, Teflon, acrylic and LPDE.

\subsubsection{MTF Measurement and Cutoff Frequency Estimation}

One possible method for assessing the MTF of a CT scanner consists of imaging a phantom containing a very small metallic spehere ${ }^{27}$; 58 . This image provides a measurement of the PSF and, through a 2D Fourier transform, the MTF. The CTP528 module (shown in Figure 17) contains two tungsten beads for exactly this purpose ${ }^{39}$. The following paragraph details this process.

There are $K$ images in a complete set of Catphan $500^{\circledR}$ images sorted as containing the tungsten bead (list "MTF"), and for the $k$-th image $(1 \leq k \leq K)$ the software applies the following algorithm:

- Find the point of maximum pixel value (the location of the bead), and select $13 \times 13$ pixels ROI around it. The ROI sizes in this and the next step are arbitrary, 
but they follow the criterion mentioned in 3.3.1 (they must always be large enough to contain the whole structure, but small enough to never overlap other structures);

- Subtract the background (the average pixel value at a $41 \times 41 \mathrm{ROI}$ at the center of the phantom, so it does not contain the bead), obtaining the $P S F_{k}(\boldsymbol{r})$;

- Calculates the system transfer function $P_{k}(f)$ taking the Fourier transform of $\operatorname{PSF}_{k}(\boldsymbol{r})$. The Fourier transform is calculated using a built-in tool of MatLab ${ }^{\circledR}$;

- According to the discussions presented in the Appendix A, the maximum frequency represented by a digital system (Nyquist frequency) is half the sampling rate. The sampling rate of a digital image is 1 point per pixel. Therefore, the frequencies $\boldsymbol{f}=\left(f_{x}, f_{y}\right)$ range from -0.5 to 0.5 pixel $^{-1}$ in both $f_{x}$ and $f_{y}$ axes (this is a general result for any digital system);

- Calculates $\operatorname{MTF}_{k}(f)$ normalizing $P_{k}(f)$ by $P_{k}(\mathbf{0})$ and taking the absolute value, as in equation (15);

- Since $\operatorname{MTF}_{k}(f)$ is radially symmetric ${ }^{57}$, it can be expressed as a function of the radial frequency $f_{r}$ alone, taking the average along the angular frequencies $f_{\varphi}$ :

$$
M T F_{k}\left(f_{r}\right)=\frac{1}{N\left(f_{r}\right)} \sum_{n=1}^{N\left(f_{r}\right)} M T F_{k}\left(f_{r}, f_{\varphi, n}\right)
$$

Where $N\left(f_{r}\right)$ is the number of pixels at a given radial frequency $f_{r}$. The uncertainty of each point $\operatorname{MTF}\left(f_{r}\right)$ is estimated as the standard deviation $\sigma_{M T F, k}\left(f_{r}\right)$ of the same $N\left(f_{r}\right)$ points that were used to estimate $M T F_{k}\left(f_{r}\right)$.

This is done for all the $K$ images, and a final estimate of $\operatorname{MTF}\left(f_{r}\right)$ and $\sigma_{M T F}\left(f_{r}\right)$ is obtained doing the weighted average over all $\operatorname{MTF}_{k}\left(f_{r}\right)$ :

$$
\begin{gathered}
M T F\left(f_{r}\right)=\sum_{k=1}^{K} \frac{M T F_{k}\left(f_{r}\right)}{\sigma_{M T F, k}^{2}\left(f_{r}\right)} / \sum_{k=1}^{K} \frac{1}{\sigma_{M T F, k}^{2}\left(f_{r}\right)} \\
\sigma_{M T F}\left(f_{r}\right)=\left(\sum_{k=1}^{K} \frac{1}{\sigma_{M T F, k}^{2}\left(f_{r}\right)}\right)^{-1 / 2}
\end{gathered}
$$


Each of the $K$ measurements $M T F_{k}\left(f_{r}\right)$ and the weighted average $\operatorname{MTF}\left(f_{r}\right)$ are saved in a ".jpeg" image file and stored in a table (".txt" file), each line containing $\left(f_{r}, \operatorname{MTF}\left(f_{r}\right), \sigma_{M T F}\left(f_{r}\right)\right)$.

Using $\operatorname{MTF}\left(f_{r}\right)$, two important frequencies are estimated. First, it estimates $f_{10 \%}$, the

frequency where $\operatorname{MTF}\left(f_{10 \%}\right)=0.1$. This frequency is often found in literature ${ }^{75 ; 76}$ as an estimative of the cutoff frequency, and is used to compare scanners performance (although other reference values exist). It is done by simple linear interpolation, taking into account the uncertainties $\sigma_{M T F}\left(f_{r}\right)$.

The second frequency is the cutoff frequency, $f_{c}$, the frequency where $\operatorname{MTF}\left(f_{c}\right) \equiv 0$. Since $\operatorname{MTF}\left(f_{r}\right)$ is an experimental result, it includes statistical errors, which means the $M T F$ is never exactly zero. Therefore, the algorithm searches for the first frequency where the $M T F$ is compatible with zero within $2 \sigma_{M T F}\left(f_{c}\right)$ (i.e., the first point compatible with zero within a $95 \%$ confidence interval). This is also done by interpolation.

Both uncertainties of $f_{10 \%}$ and $f_{c}$ are calculated only propagating the uncertainty of the 2 $M T F$ points used in their respective interpolations.

It is also important to notice that $f_{c}$ is calculated from points in a region where the $M T F$ fluctuates very close to zero. Therefore, the accurate estimation of this frequency is hard and the uncertainty of $f_{c}$ can be very sensitive to over or underestimation. 


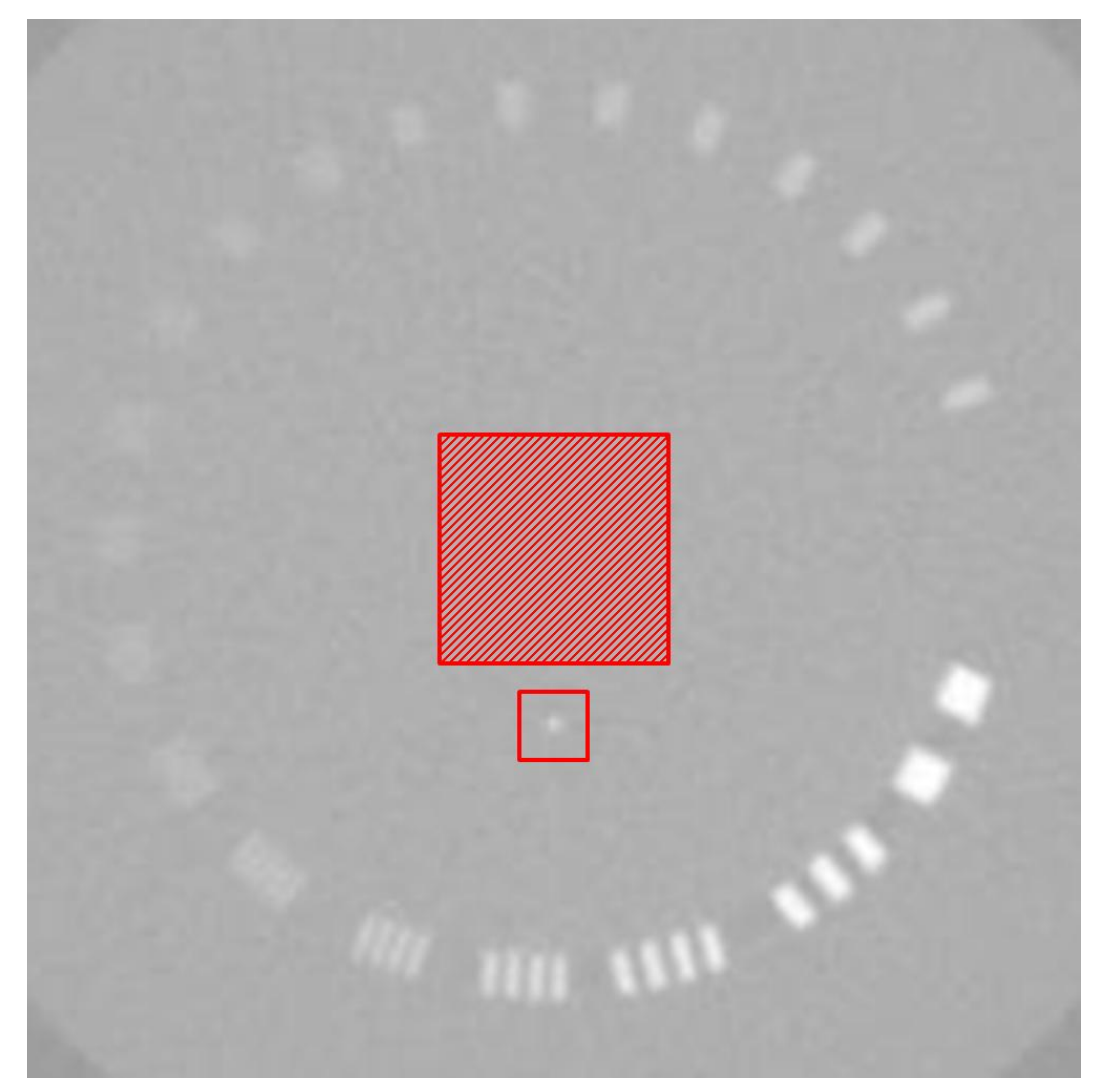

Figure 17: amplified CT image of the CTP528 module of the Catphan500 ${ }^{\circledR}$ phantom. The blank red box represents the ROI selected around the tungsten bead. The striped red box represents the ROI used to calculate the background.

\subsection{7. $\quad$ Noise and NPS}

The software calculates image noise by taking the standard deviation in 4 peripheral ROI's and 1 central ROI (each $61 \times 61$ pixels) for each image marked as having no structure (list "noise"). This is saved as a text file.

There are multiple possible procedures to calculate NPS, and each one produces significantly different results ${ }^{77}$. The procedure adopted in this software follows the ICRU Report $\# 87^{44}$ recommendations. The phantom needs to be scanned at least twice, because this procedure requires at least one pair of images representing the same section of the phantom (the algorithm to find pairs of these images is explained in Appendix D). First, the software finds pairs of images taken at the same table position (and therefore representing the same section of the phantom). If there are no pairs in the image set, the program does not calculate NPS. If there is at least one pair, for the $l$-th pair $(1 \leq l \leq$ $L)$ :

- Subtract both images and corrects by the $\sqrt{2}$ factor to keep the same noise magnitude: 


$$
\mathrm{x}_{l}(\boldsymbol{r})=\frac{\mathrm{x}_{l, 1}(\boldsymbol{r})-\mathrm{x}_{l, 2}(\boldsymbol{r})}{\sqrt{2}}
$$

- Select $M$ ROI's symmetrically disposed around the center of the phantom (size $61 \times 61$ pixels);

- $\quad$ For the $m$-th ROI $(1 \leq m \leq M)$ :

Subtract the mean of $\mathrm{x}_{l, m}(\boldsymbol{r})$, turning it into a zero-mean variable:

$$
\Delta \mathrm{x}_{l, m}(\boldsymbol{r})=\mathrm{x}_{l, m}(\boldsymbol{r})-\left\langle\mathrm{x}_{l, m}(\boldsymbol{r})\right\rangle_{\boldsymbol{r}}
$$

Calculates the square of the absolute value of the Fourier transform of $\Delta \mathrm{x}_{l, m}(\boldsymbol{r})$, as in equation (21):

$$
\left|\Delta \mathrm{X}_{l, m}(\boldsymbol{f})\right|^{2}=\left|\widetilde{\mathcal{F}_{k}}\left[\Delta \mathrm{x}_{l, m}(\boldsymbol{r})\right]\right|^{2}
$$

$>\left|\Delta \mathrm{X}_{l, m}(\boldsymbol{f})\right|^{2}$ is a "partial" NPS estimative, calculated for only the $m$-th ROI of the $l$-th image pair;

$>$ The frequencies $\boldsymbol{f}$ range from -0.5 to 0.5 pixel $^{-1}$ in both $f_{x}$ and $f_{y}$ axes.

- The average over all $M$ ROI's is taken, obtaining the estimative of NPS of the $l$-th image pair:

$$
N P S_{l}(\boldsymbol{f})=\left\langle\left|\Delta \mathrm{X}_{l, m}(\boldsymbol{f})\right|^{2}\right\rangle_{m}=\frac{1}{M} \sum_{m=1}^{M}\left|\Delta \mathrm{X}_{l, m}(\boldsymbol{f})\right|^{2}
$$

- Similarly to the $M T F, N P S$ is radially symmetric, so it can be expressed as a function of $f_{r}$ in an analogous way:

$$
N P S_{l}\left(f_{r}\right)=\frac{1}{N\left(f_{r}\right)} \sum_{n=1}^{N\left(f_{r}\right)} N P S_{l}\left(f_{r}, f_{\varphi, n}\right)
$$


For a better estimative of the noise produced by a CT scanner using a particular acquisition setup, an average over the $L$ image pairs can be done, analogously to what is done for calculating the $M T F$ :

$$
\begin{gathered}
N P S\left(f_{r}\right)=\sum_{l=1}^{L} \frac{N P S_{l}\left(f_{r}\right)}{\sigma_{N P S, l}^{2}\left(f_{r}\right)} / \sum_{l=1}^{L} \frac{1}{\sigma_{N P S, l}^{2}\left(f_{r}\right)} \\
\sigma_{N P S}\left(f_{r}\right)=\left(\sum_{l=1}^{L} \frac{1}{\sigma_{N P S, l}^{2}\left(f_{r}\right)}\right)^{-1 / 2}
\end{gathered}
$$

All $N P S_{l}(\boldsymbol{f})$ are saved as jpeg images and data tables in text files. All $N P S_{l}\left(f_{r}\right)$ plus $\operatorname{NPS}\left(f_{r}\right)$ are also saved as jpeg graphs and as tables in text files, where each line contains $\left(f_{r}, N P S\left(f_{r}\right), \sigma_{N P S}\left(f_{r}\right)\right)$.

\subsection{Software Validation}

The images used to validate the software were acquired by the quality control team of Grupo de Dosimetria das Radiações e Física Médica do Instituto de Física da Universidade de São Paulo (GDRFM/IFUSP), using CT scanners of three institutions: Instituto de Radiologia do Hospital das Clínicas da Faculdade de Medicina da Universidade de São Paulo (INRAD/FMUSP), Instituto do Câncer do Estado de São Paulo Octávio Frias de Oliveira (ICESP) and Serviço de Verificação de Óbitos da Faculdade de Medicina da Universidade de São Paulo (SVO/FMUSP).

The goal of this work was to develop a tool (the software) to improve the workflow of quality control programs in CT. Therefore, the effectiveness of this tool is required to be checked against the procedures adopted nowadays.

The results from the software were compared to the results obtained by the GDRFM qualified experts proficient in quality control (QC, for short) for different CT protocols. This comparison employed statistical hypothesis tests, whenever possible. The methodology of these comparisons is described in the following subsections. All the calculations performed for the software validation were executed in Microsoft Excel ${ }^{\circledR}$, and the graphs were created using the software Origin $8^{\circledR}$. 


\subsubsection{Pixel Size, Slice Thickness, Increment and CT Number Calibration}

For pixel size, slice thickness, slice increment and CT number calibration coefficients the comparison between experts and software results is straightforward. It is only necessary to compare the values obtained by the software against those obtained by the QC. To do this, the t-test was applied ${ }^{78}$; this test compares two mean values $\overline{x_{1}}$ and $\overline{x_{2}}$, each obtained independently with uncertainties $\sigma_{1}$ and $\sigma_{2}$, and number of data points $n_{1}$ and $n_{2}$ respectively:

$$
\begin{gathered}
\sigma^{2}=\frac{\sigma_{1}^{2}\left(n_{1}-1\right)+\sigma_{2}^{2}\left(n_{2}-1\right)}{n_{1}+n_{2}-2} \\
t=\frac{\overline{x_{1}}-\overline{x_{2}}}{\sigma \sqrt{\frac{1}{n_{1}}+\frac{1}{n_{2}}}}
\end{gathered}
$$

In this particular case, pixel size, slice thickness, slice increment and the CT number fitting coefficients are all calculated with four data points, by the software and by the QC; therefore, $n_{1}=n_{2}=4$. So, the critical value of $t$ is $t_{\text {crit }}=3.71$, for 6 degrees-offreedom and $p=0.01$ (i.e., if $|t|>t_{\text {crit }}$, the hypothesis that the 2 values being compared are equal is unlikely to be true). The Appendix B presents details about the ttest.

It is expected that about 1 in 100 times $|t|>3.71$ (i.e., the value calculated by the software will appear to be significantly different from the QC). For all other obtained values, $|t| \leq 3.71$ (the two values are accepted as being equal). If the $t$ values obtained follow this behavior, the software results for these variables are comparable with the QC results.

Not all values resulting from the QC reports had uncertainties. Other times these uncertainties were an order of magnitude lower than in most reports, making the result suspect. In these cases, the relative uncertainty of the QC value was assumed to be in the same order of magnitude of the uncertainties considered reliable. The exception is the coefficients of the CT number calibration, which had no uncertainty values in any report. The linear and angular coefficients are supposed to be compatible with zero and one respectively. Therefore, the relative uncertainties of the linear and angular 
coefficients were supposed to be sufficiently high in order to have these coefficients compatible with zero and one respectively, within a $95 \%$ confidence interval.

\subsubsection{Noise Magnitude}

The noise magnitude, $\sigma$, cannot be compared using the t-test because it is standard deviation and not a mean value. So, instead of the t-test, the Fisher F-test ${ }^{79}$ was applied. This test consists of calculating the quotient of the 2 variances being compared:

$$
F=\frac{\sigma_{1}^{2}}{\sigma_{2}^{2}}
$$

This quantity follows the Fisher distribution, so it has mean and variance equal to:

$$
\begin{gathered}
\langle F\rangle=\frac{v_{2}}{v_{2}-2} \\
\sigma_{F}^{2}=\frac{2 v_{2}^{2}\left(v_{1}+v_{2}-2\right)}{v_{1}\left(v_{2}-2\right)^{2}\left(v_{2}-4\right)}
\end{gathered}
$$

Where $v_{1}$ and $v_{2}$ are the number of degrees-of-freedom of $\sigma_{1}^{2}$ and $\sigma_{2}^{2}$ respectively. The Appendix B presents details regarding the F-test.

The value $v_{1}$ is the number of degrees of freedom from the software noise calculation, which is known. As explained in section 3.3.7, noise is calculated in a 61x61 ROI, so:

$$
v_{1}=61^{2}-1=3720
$$

The value $v_{2}$ is the number of degrees-of-freedom from the QC noise calculation, which is not known (and may vary from one analysis to another, because the ROI selection is made manually). Therefore it was supposed to be equal to $v_{1}$, since it is expected to be at least in the same order of magnitude.

Given $v_{1}=v_{2}=v=3720 \gg 1$, the formulas for mean and variance of the $F$ quantity approximate to: 


$$
\begin{gathered}
\langle F\rangle \approx 1 \\
\sigma_{F}^{2} \approx \frac{4}{v}=0.0011
\end{gathered}
$$

So, the probability $p$ of a value being outside the interval $\langle F\rangle \pm 2 \sigma_{F}$ is:

$$
\begin{gathered}
p=1-P\left[\langle F\rangle-2 \sigma_{F} \leq F \leq\langle F\rangle+2 \sigma_{F}\right]=1-\int_{\langle F\rangle-2 \sigma_{F}}^{\langle F\rangle+2 \sigma_{F}} \rho(F, v, v) d F \\
p \approx 0.046
\end{gathered}
$$

In the equation (78), $\rho(F, v, v)$ is the Fisher distribution.

It means it is expected that about 1 in 20 times the $F$ value will be outside the interval $[0.934,1.066]$. In this case, the noise calculated by the software will appear to be significantly different from the QC. In the other cases, $F$ will be evaluated within the interval (the difference has low significance). If the $F$ values obtained follow this behavior, the software noise results are comparable with the QC noise results.

\subsubsection{Cutoff Frequency}

As explained in section 3.3.6, the software estimates two different frequencies from the measured $M T F$ : the frequency of the first zero of the $M T F, f_{c}$, and the frequency where the $M T F$ is $0.1, f_{10 \%}$. The first is the definition of the cutoff frequency, the last frequency that the system can represent. The second is sometimes found in the literature ${ }^{75 ; 76}$ as an estimator of the cutoff frequency, although it has a different definition. Since they have different definitions, the obtained values for each one are not necessarily expected to be compatible with each other.

The conventional QC program estimates, by a visual criterion, the last bar pattern (and its spatial frequency) of CTP528 module where each bar can still be distinguished. This frequency is here labeled $f_{L D}$ (frequency of the Last Distinguishable bar pattern). This frequency is not necessarily the limit spatial of resolution of the system (the cutoff frequency). The limit of resolution is between this frequency and another, $f_{n e x t}$, the frequency of the next bar pattern, where the bars are not distinguishable. This is 
illustrated in Figure 18. Both frequencies are given by the Catphan $500^{\circledR}$ user manual, in $\mathrm{mm}^{-1}$, and then they are converted to pixel $^{-1}$ to be represented in the same unit as $f_{c}$ and $f_{10 \%}$. Assuming a constant distribution and using $f_{L D}$ and $f_{\text {next }}$, the best estimative of the spatial resolution limit given by the $\mathrm{QC}$ reports $\left(f_{Q C}\right)$ and its uncertainty are:

$$
\begin{gathered}
f_{Q C}=\frac{f_{L D}+f_{\text {next }}}{2} \\
\sigma_{f Q C}=\frac{f_{\text {next }}-f_{L D}}{\sqrt{12}}
\end{gathered}
$$

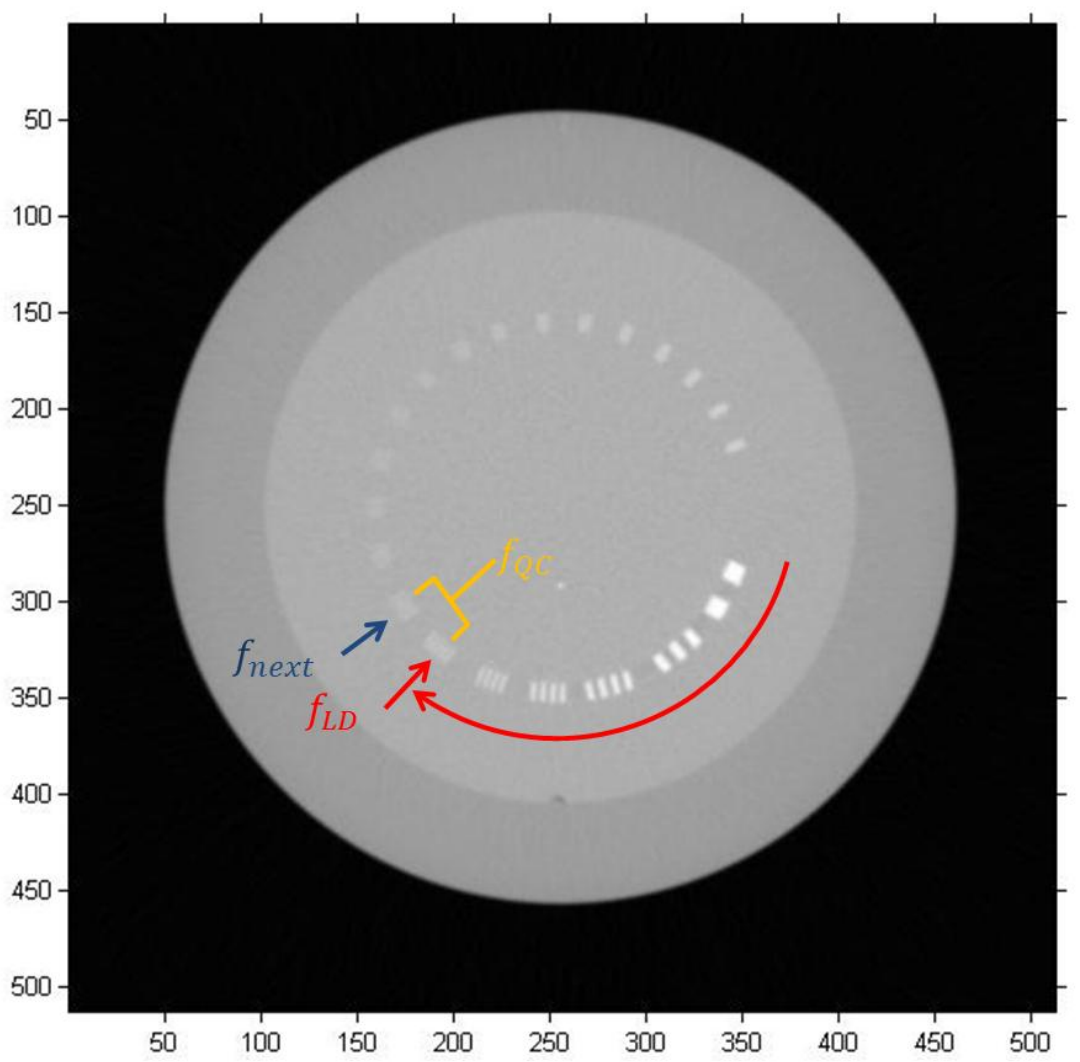

Figure 18: CT image representing the CTP528 Catphan $500^{\circledR}$ module, illustrating the process of obtaining the QC estimation of the cutoff frequency ( $f_{Q C}$, in yellow). Following the clockwise red arrow, the last pattern where the bars are visually still distinguishable is found and its spatial frequency $f_{L D}$ (in red) is recorded. In the next pattern, of spatial frequency $f_{\text {next }}$ (in blue), the bars are not distinguishable anymore. The actual estimative of the cutoff frequency is between these two frequencies and has constant distribution.

The two equations above result from the assumption of a constant distribution for $f_{Q C}$. 
The frequencies $f_{c}$ and $f_{Q C}$ are both cutoff frequency estimates. However, $f_{c}$ refers to estimated considering the CT system independently, whereas $f_{Q C}$ refers to the CT system plus other factors such as the human vision of the observer, visualization tools (monitor), environment illumination, etc. Therefore, $f_{c}$ and $f_{Q C}$ have different definitions, and their values might differ as well.

Since all these three frequencies have different definitions, the t-test is not applicable. So, these three frequencies $\left(f_{10 \%}, f_{c}\right.$ and $\left.f_{Q C}\right)$ were plotted in a single graph and interpreted qualitatively.

\subsection{4. $M T F$ and NPS}

The MTF and NPS functions are not routinely measured in QC program. Therefore, software results cannot be compared to values reported by these programs. However, they can be checked through the theory demonstrated in section 2.3.

Equation (53) shows a relation between the NPS $\left(W_{\widehat{\Delta s}}(\boldsymbol{f})\right)$ and MTF $(J(f))$ of the reconstruction algorithm (the constants were compacted in the factor $a$ ):

$$
W_{\widehat{\Delta s}}(\boldsymbol{f})=a . f|J(f)|^{2}
$$

The measured MTF and NPS functions correspond to the whole CT system with all its parts. However, the above equation relates only the MTF of the reconstruction algorithm to the respective NPS. Therefore, before using this equation, it must be adapted.

Equation (18) shows that $M T F$ functions of each part of a system stack multiplicatively. So, multiplying both sides of the equality by the squared MTF of all other unaccounted (and unknown) parts:

$$
\begin{gathered}
W_{\widehat{\Delta s}}(\boldsymbol{f})\left|M T F_{\text {other }}(\boldsymbol{f})\right|^{2}=a \cdot \boldsymbol{f}|J(\boldsymbol{f})|^{2}\left|M T F_{\text {other }}(\boldsymbol{f})\right|^{2} \\
N P S(\boldsymbol{f})=a \cdot \boldsymbol{f}|M T F(\boldsymbol{f})|^{2}
\end{gathered}
$$

So, Equation (53) is also valid for whole-system MTF and NPS. Taking the natural logarithm of both sides, and expressing everything one-dimensionally as function of the radial frequency: 


$$
\begin{gathered}
\ln \left(N P S\left(f_{r}\right)\right)=\ln (a)+\ln \left(f_{r}\right)+2 \ln \left(\left|M T F\left(f_{r}\right)\right|\right) \\
\ln \left(N P S\left(f_{r}\right)\right)=\ln (a)+b \cdot \ln \left(f_{r}\right)+c \cdot \ln \left(\left|M T F\left(f_{r}\right)\right|\right)
\end{gathered}
$$

So, the constants $\ln (a), b$ and $c$ can be fitted linearly, and it is expected that $b=1$ and $c=2$. Figure 19 shows an example of this fit.

The calculated NPS presents a larger number of data points than the calculated MTF. Because of this, before the fitting, it was calculated a NPS curve only at the same frequencies as the MTF curve (using a moving average to smooth the curve and then linear interpolation).

The weights chosen for the fit are:

$$
w_{i}=\left(\frac{N P S\left(f_{r, i}\right)}{\max _{i}\left(N P S\left(f_{r, i}\right)\right)}\right)
$$

The reduced chi-square $Q_{r e d}$, used to evaluate the quality of the fit, was calculated as:

$$
Q_{\text {red }}=\frac{1}{v}(\boldsymbol{y}-\widehat{\boldsymbol{y}})^{\prime} \mathbf{V}^{-1}(\boldsymbol{y}-\widehat{\boldsymbol{y}})
$$

Where $v$ is the number of degrees of freedom (data points minus fitted parameters), $y_{i}=\ln \left(N P S\left(f_{r, i}\right)\right)$ using the experimental points of $N P S, \hat{y}_{i}=\ln \left(N P S\left(f_{r, i}\right)\right)$ calculated from the MTF with the fitted parameters, and $\mathbf{V}$ is the variance matrix of $\boldsymbol{y}$. A low $Q_{\text {red }}$ indicates only overestimated uncertainties; a high $Q_{\text {red }}$ may indicate underestimated uncertainties or a bad fitting function. Then, as the objective is to assess if the data comply to the theoretical relation, only the right tail (high values) of the $\chi_{\text {red }}^{2}$ distribution was rejected in the chi-square test ${ }^{80}$ (chosen p-value of 0.05 ):

$$
\int_{0}^{Q_{\text {red,Crit }}} \chi_{\text {red }}^{2}\left(Q_{\text {red }} ; v\right) d Q_{\text {red }}=1-p
$$


So, if a fit has a $Q>Q_{\text {red,Crit }}$ it will be rejected, and the chance of this happening is $5 \%$, or 1 in 20 .
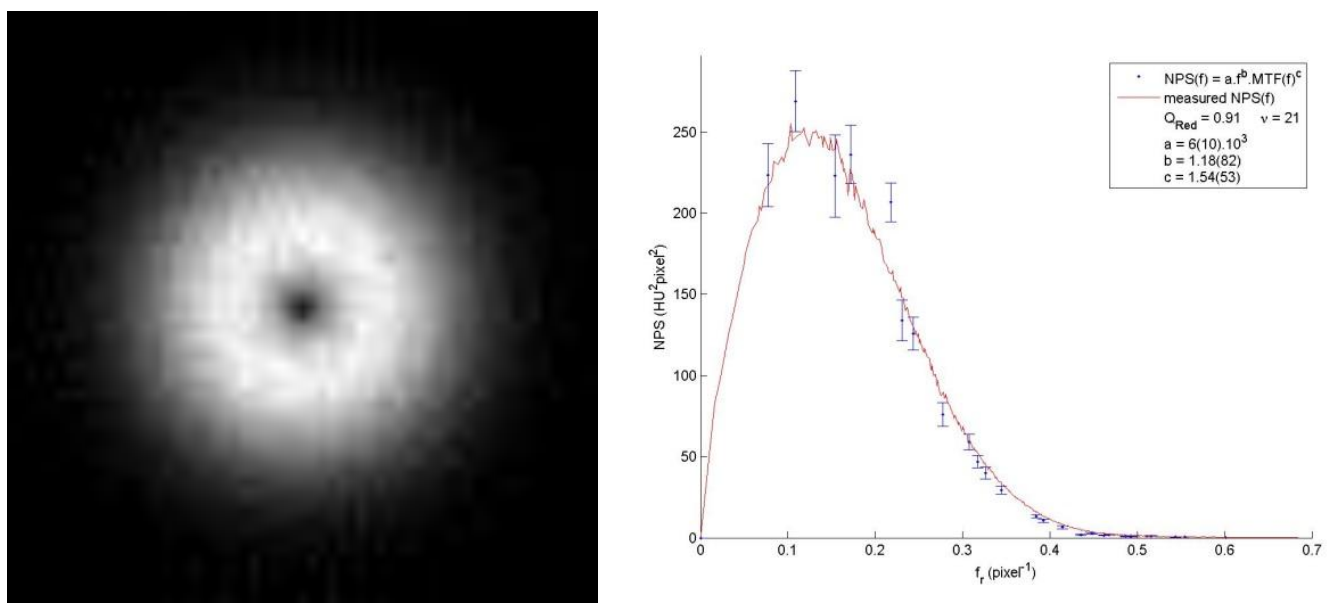

Figure 19: Example of measured NPS, using a Phillips Brilliance16 CT scanner, in axial mode and selecting 1.5mm of slice thickness. (a) 2D NPS. (b) NPS expressed one-dimensionally (red line) and fitted as a function of the MTF (blue points).

\subsection{CT Images for Software Validation}

Ten different CT machine models from four different vendors are currently used in INRAD, ICESP and SVO. Catphan $500^{\circledR}$ image sets were obtained by the GDRFM experts using these scanners, following a number of different protocols (user defined settings), as show in Table 1. These sets were obtained during the years of 2013 and 2014, as part of the regular GDRFM quality control program. Therefore, the results obtained by the developed software were compared, whenever possible, with the results presented in quality control reports (see section 3.4).

Table 1: scanners used to test the software, separated by hospital, manufacturer and model.

\begin{tabular}{|c|c|c|c|}
\hline Institution & Manufacturer & Model & $\begin{array}{c}\text { Number of } \\
\text { Protocols }\end{array}$ \\
\hline SVO & Siemens & Somatom Emotion16 & 2 \\
\hline \multirow{4}{*}{ INRAD } & Toshiba & Aquillion & 3 \\
\hline & \multirow{3}{*}{ GE } & LightSpeed & 3 \\
\hline & & Discovery 750 & 3 \\
\hline & & BrightSpeed & 2 \\
\hline \multirow{4}{*}{ ICESP } & \multirow{4}{*}{ Philips } & Brilliance16 & 2 \\
\hline & & Brilliance 40 & 3 \\
\hline & & Brilliance64 & 3 \\
\hline & & Brilliance BigBore & 4 \\
\hline
\end{tabular}




\subsection{Software Limitations}

By its design, the software imposes some constrains on the image acquisition process. First, the chosen FOV must be close to $250 \mathrm{~mm}$. Some parts of the code use fixed numbers to locate regions of the phantom. Changing the FOV changes the apparent size of the phantom in the image, and therefore changes the position of each structure in the images. The software was created and tested with $250 \mathrm{~mm}$ FOV images as reference and, although it has some tolerance for inconsistency at these structure locations (as described in section 3.3.1), using a radically different FOV during the image acquisition will cause the software to not work properly. Some of the protocols used have slightly different FOV values (240 and $235 \mathrm{~mm}$ ) and the software demonstrates it still works properly. It is recommended selecting a FOV of $250 \pm 15 \mathrm{~mm}$.

For a similar reason, the phantom must be well aligned in the CT scanner (parallel with the z-axis), and must not be too dislocated from the center of the scan field. If the phantom is dislocated in the $x-y$ plane from the center of the image by more than 15 pixels (half the ROI size used by the routine described in section 3.2.2), the alignment pins will be outside the defined ROI's, and the software will not recognize them. If the phantom is inclined with relation to the $\mathrm{z}$-axis by more than approximately $1^{\mathrm{o}}$, one or more of the pins may not be represented in the image. In both cases the automatic identification of the phantom slices will not work.

In addition, the software depends on having images of the CTP401 section to find all structures inside the phantom. If these images are not available but the other sections are, the identification algorithm will not find them and they will not place them in their respective lists. In this case, this task will have to be done manually by the user (the software interface has buttons for that in case it is necessary). So, it is advised to always scan the whole phantom, never only a part of it. Anyway, it is already the usual procedure adopted by Catphan $500^{\circledR}$ users.

Lastly, the NPS calculation is only possible with image pairs of the exact same uniform section (table position). So, it is worthy to always scan the phantom at least twice. If only one scan is performed, only the noise magnitude will be calculated. The procedure for image pair recognition is detailed on Appendix D.

It is worth noting that none of these conditions are very restrictive. Quality control programs already take great care to correctly position and align the phantoms when 
acquiring data, so misplacing the phantom should be a rare occurrence. The $250 \mathrm{~mm}$ FOV is adequate to scan the Catphan500 phantom: with a FOV much smaller the scan would only cover a part of the phantom, and with a FOV much larger the phantom would appear very small in the image, most of the image would be air. Lastly, the requirement of two whole-phantom scans does not demand any extra work. It takes only a few more seconds than a single scan. 


\section{RESULTS AND DISCUSSION}

Section 4.1 presents and discusses the results of the software validation process, using statistical tests in most cases. Section 4.2 presents an evaluation of the software performance in terms of the time needed by the functions "Sort" and "Identify" to process a set of images.

\subsection{Validation}

Table 2 numbers the protocols used. This numbering is used in all graphs in this section. The subsections 4.1.1 to 4.1.3 present the comparison of results between the software and the quality control. Subsection 4.1.4 presents the fitting of MTF and NPS obtained by the software. Notice that, since all the image acquisitions were made before this work started, not all of them were performed with the intent of calculating the NPS function. Therefore, only 13 of the 27 protocols had the NPS evaluated.

\subsubsection{Pixel Size, Slice Thickness, Increment and CT Number Calibration}

As discussed in the item 3.4.1, the Student's t-test was applied to compare the results from the software and from the QC.

Figures 18 to 20 present the slice thickness results. The first graph shows the slice thickness obtained by the software and the QC at each protocol, sorted by scanner. The second presents the percentual difference between the software and QC results. The third shows the t-test results, with the acceptance limits delimited by the red dashed line. Figures 21 to 30 show the results for the slice increment, pixel size, and the linear and angular coefficients from the CT number calibration (for the calibration coefficients the percentual difference is irrelevant, so this graph is not presented). 
Table 2: List of the protocols used to validate the software. The numbering in the first column is the same used in the graphs in the next subsections. The second column shows the scanner model and manufacturer. The third column presents the acquisition mode (axial or helical). The fourth column presents the reconstruction algorithm used. The fifth column gives the name of the applied filter (as it is in the DICOM header). The sixth column presents the selected nominal slice thickness (in mm). The last column presents the DFOV selected (also in mm). Each group of protocols with the same scanner is separated from the others by a bold line. An asterisk beside the protocol number indicates this is one of the protocols where the calculation of the NPS was possible.

\begin{tabular}{|c|c|c|c|c|c|c|}
\hline $\begin{array}{l}\text { Protocol } \\
\text { Number }\end{array}$ & $\begin{array}{l}\text { Scanner Model } \\
\text { (Manufacturer) }\end{array}$ & $\begin{array}{l}\text { Acquisition } \\
\text { Mode }\end{array}$ & $\begin{array}{c}\text { Reconstruction } \\
\text { Algorithm }\end{array}$ & Filter Name & $\begin{array}{c}\text { Nominal } \\
\text { Slice } \\
\text { Thickness } \\
\text { (mm) }\end{array}$ & $\begin{array}{c}\text { DFOV } \\
(\mathbf{m m})\end{array}$ \\
\hline 1 & Aquillion (Toshiba) & Axial & FBP & FC70 Small & 0.5 & 240 \\
\hline $2 *$ & Aquillion (Toshiba) & Axial & FBP & FC70 Small & 1.0 & 240 \\
\hline 3 & Aquillion (Toshiba) & Helical & $-i$ & FC70 Small & 1.0 & 240 \\
\hline 4 & Brightspeed (GE) & Helical & FBP & Soft Body & 1.25 & 250 \\
\hline 5 & Brightspeed (GE) & Axial & FBP & Standard Head & 2.5 & 250 \\
\hline $6 *$ & Brilliance16 (Philips) & Axial & FBP & B B & 1.5 & 250 \\
\hline 7 & Brilliance16 (Philips) & Helical & FBP & UB UB & 2.0 & 250 \\
\hline $8 *$ & Brilliance40 (Philips) & $\overline{\text { Axial }}$ & $\overline{\text { FBP }}$ & EB EB & 1.25 & 250 \\
\hline 9 & Brilliance40 (Philips) & Helical & FBP & UB UB & 2.0 & 250 \\
\hline 10 & Brilliance40 (Philips) & Helical & FBP & B B & 3.0 & 250 \\
\hline $11 *$ & $\begin{array}{l}\text { BrillianceBigBore } \\
\text { (Philips) }\end{array}$ & Axial & FBP & B B & 1.5 & 250 \\
\hline 12 & $\begin{array}{l}\text { BrillianceBigBore } \\
\text { (Philips) }\end{array}$ & Helical & FBP & UB UB & 3.0 & 250 \\
\hline 13 & $\begin{array}{l}\text { BrillianceBigBore } \\
\text { (Philips) }\end{array}$ & Helical & FBP & B B & 2.0 & 250 \\
\hline 14 & $\begin{array}{l}\text { BrillianceBigBore } \\
\text { (Philips) }\end{array}$ & Helical & FBP & $\mathrm{C} \mathrm{C}$ & 1.25 & 250 \\
\hline $15 *$ & Discovery690 (GE) & $\overline{\text { Axial }}$ & FBP & Standard Medium & 1.25 & 250 \\
\hline $16 *$ & Discovery690 (GE) & Axial & FBP & Standard Medium & 2.5 & 250 \\
\hline $17 *$ & Discovery750 (GE) & Axial & FBP & Standard Medium & 1.25 & 250 \\
\hline $18 *$ & Discovery750 (GE) & Helical & ASIR SS50ii & Soft Body & 2.5 & 250 \\
\hline 19 & Discovery750 (GE) & Helical & ASIR SS50ii & Standard Medium & 0.625 & 250 \\
\hline 20 & $\begin{array}{l}\text { Somatom Emotion } 16 \\
\text { (Siemens) }\end{array}$ & $-{ }^{i}$ & FBP & $50 \mathrm{~B} 41 \mathrm{~s} 8$ & 5.0 & 250 \\
\hline $21 *$ & $\begin{array}{l}\text { Somatom Emotion } 16 \\
\text { (Siemens) }\end{array}$ & $-{ }_{-}^{i}$ & FBP & $\mathrm{H} 31 \mathrm{~s} 1$ & 1.2 & 250 \\
\hline $22 *$ & LightspeedUltra (GE) & Axial & FBP & Standard Head & 1.25 & 240 \\
\hline 23 & LightspeedUltra (GE) & Axial & FBP & Standard Head & 1.25 & 250 \\
\hline $24 *$ & LightspeedUltra (GE) & Axial & FBP & Standard Head & 2.5 & 250 \\
\hline $25 *$ & Brilliance64 (Philips) & Axial & FBP & $\bar{B}$ B & 1.25 & 250 \\
\hline $26 *$ & Brilliance64 (Philips) & Axial & FBP & B B & 2.5 & 250 \\
\hline 27 & Brilliance64 (Philips) & Helical & FBP & UB UB & 2.0 & 235 \\
\hline
\end{tabular}

${ }^{i}$ this information is not simply identified in the DICOM header of this specific CT scanner

ii "SS50" means the image reconstruction used a combination of 50\% FBP and 50\% ASIR 


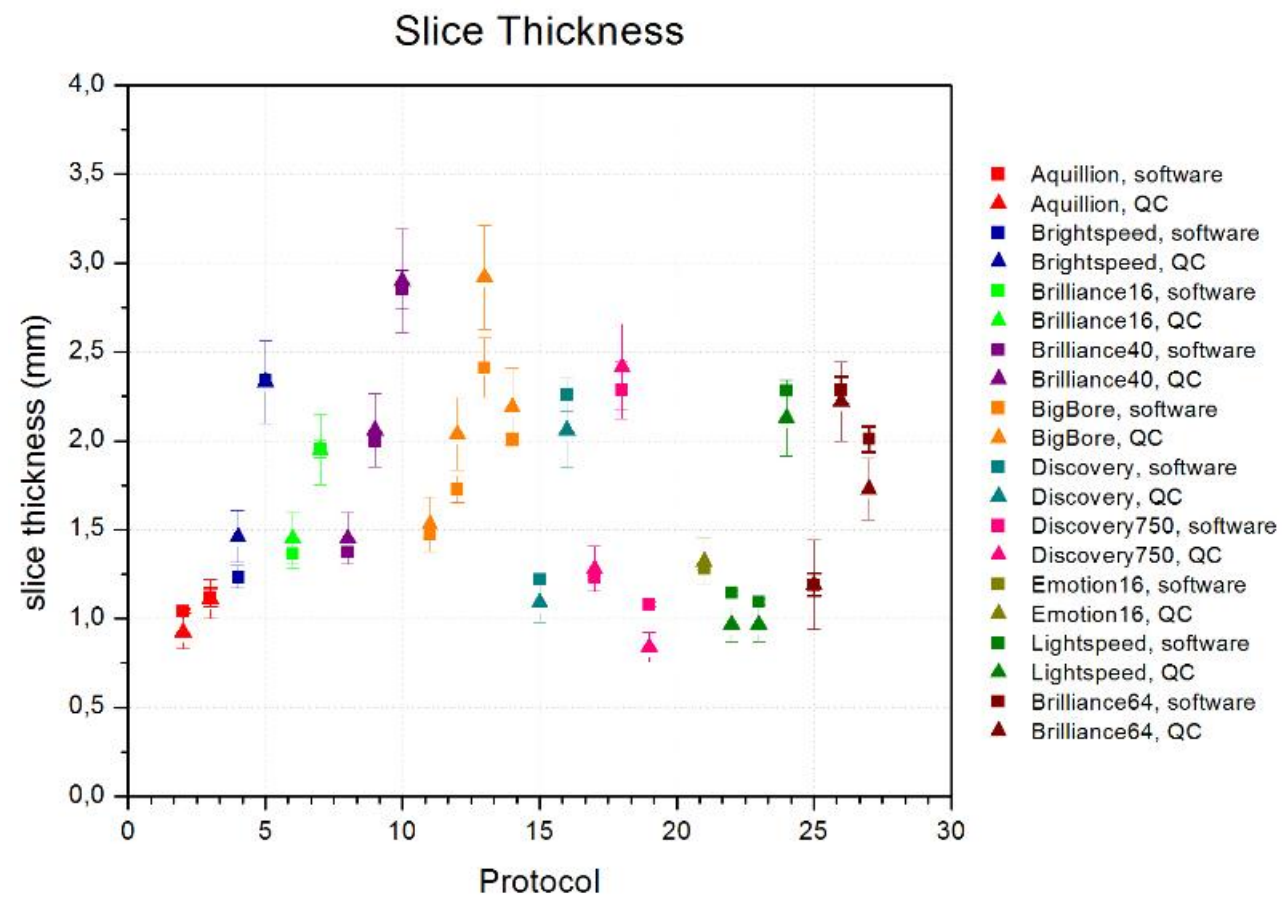

Figure 20: Slice thickness obtained by the software (squares) and the QC (triangles) for each protocol, sorted by scanner (different colors).

Slice Thickness - percentual difference Software-QC

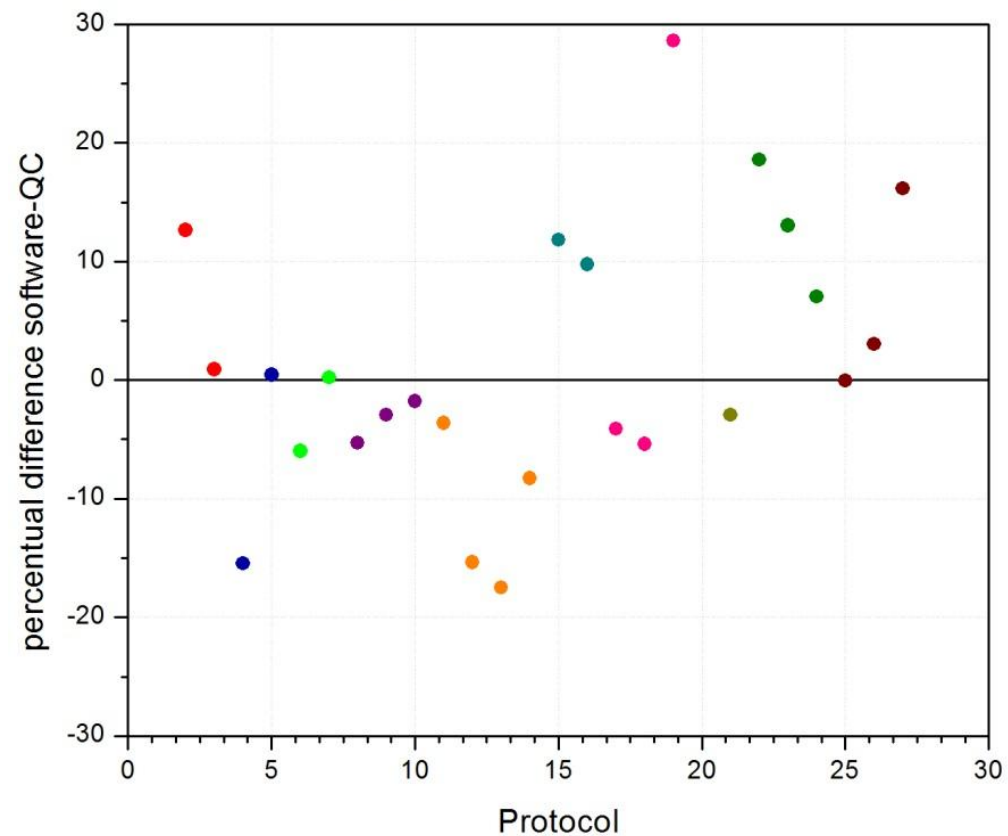

- Aquillion

- Brightspeed

- Brilliance16

- Brilliance40

- BigBore

- Discovery

- Discovery 750

- Emotion16

- Lightspeed

- Brilliance64

Figure 21: Percentual difference between the software and the QC slice thickness results in each protocol, sorted by scanner (different colors). 


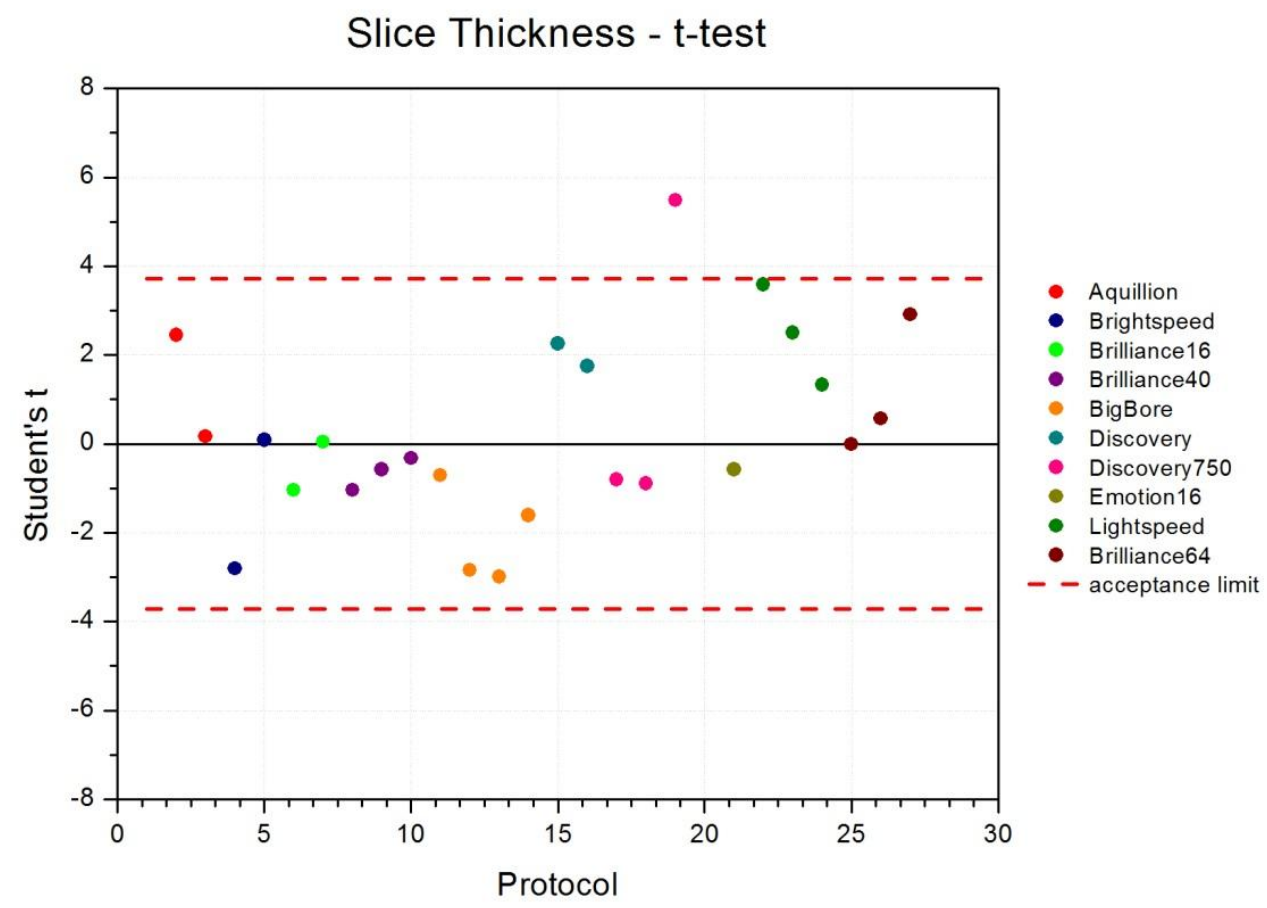

Figure 22: Student's $t$ value for slice thickness for each protocol, sorted by scanner (different colors). The red dashed lines represent the acceptance interval. Only protocol 19 is outside the acceptance interval.

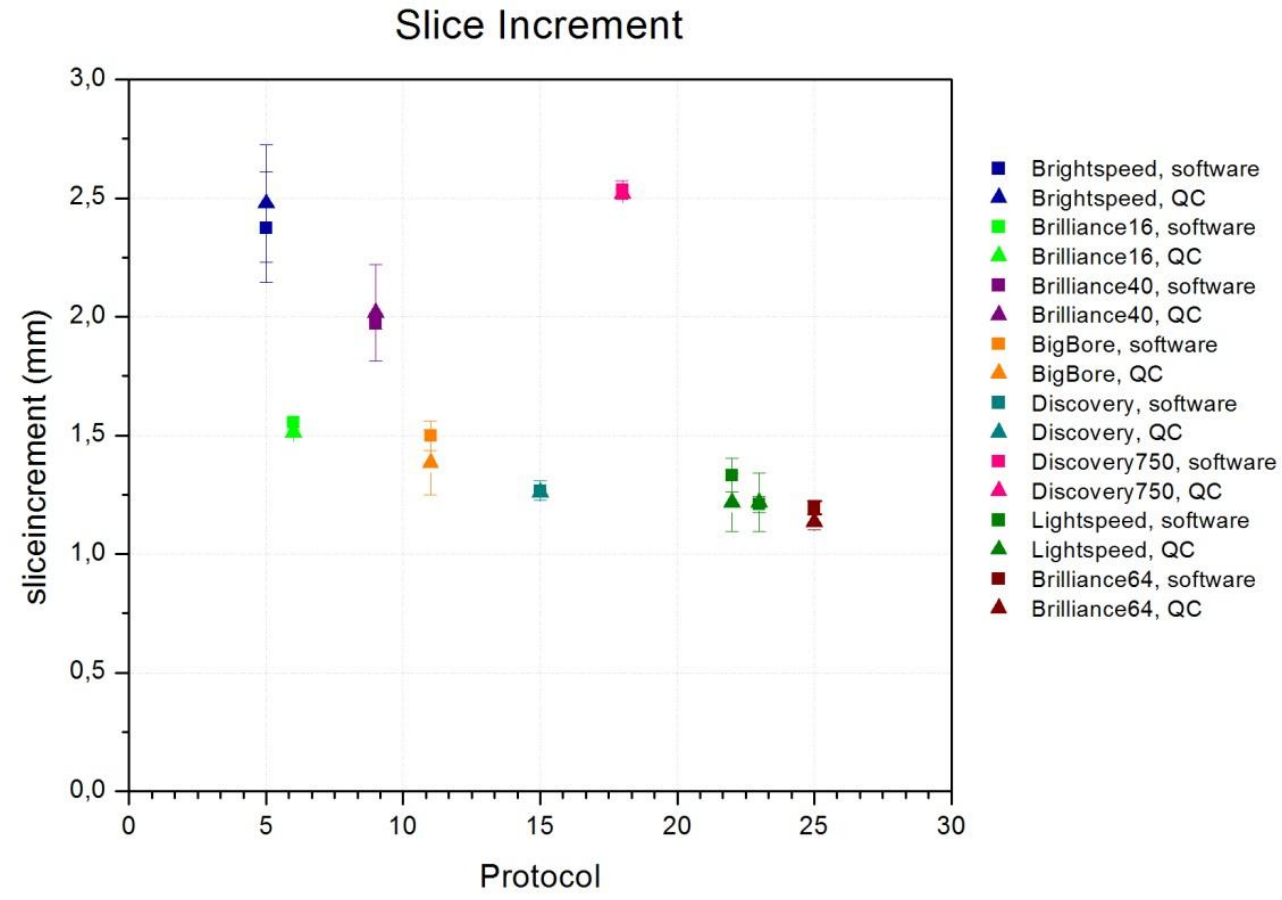

Figure 23: Slice increment obtained by the software (squares) and the QC (triangles) for each protocol, sorted by scanner (different colors). 


\section{Slice Increment - percentual difference Software-QC}

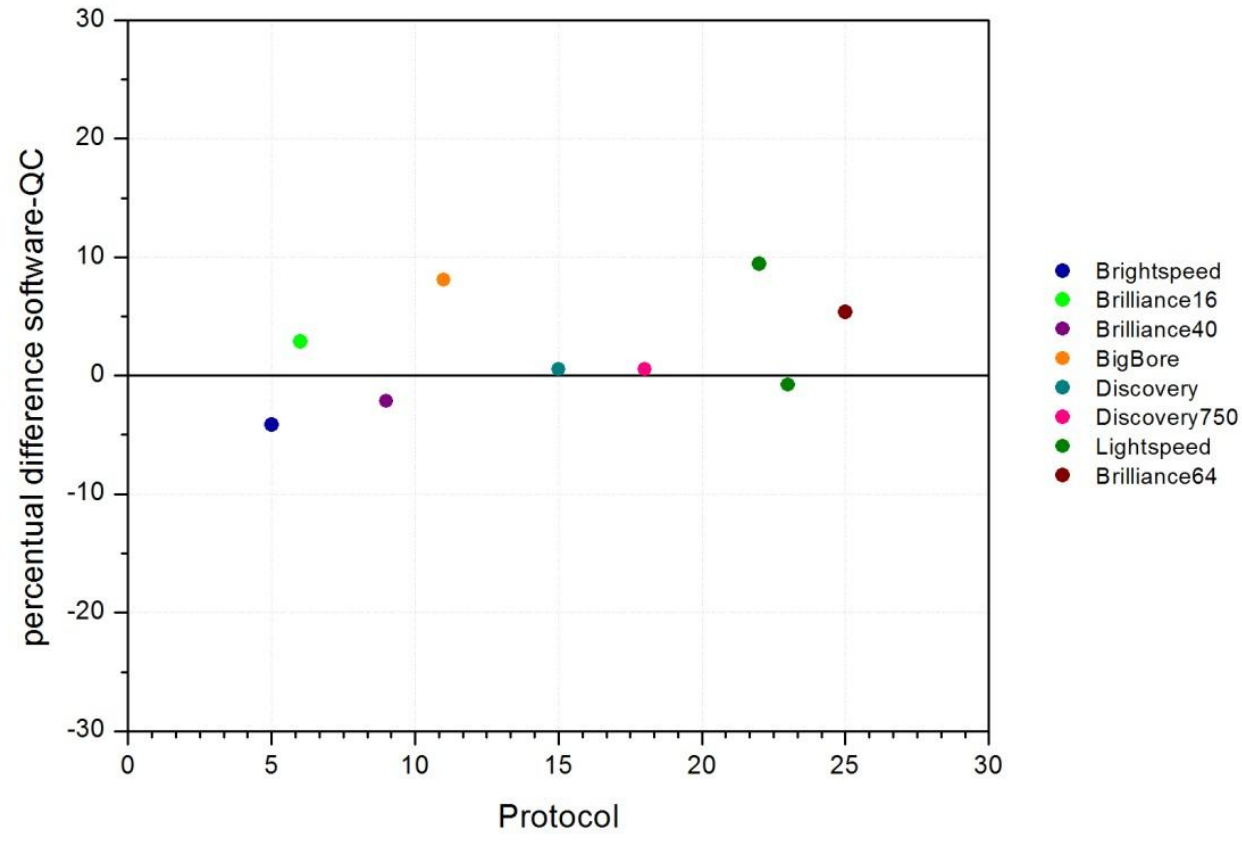

Figure 24: Percentual difference between the software and the QC slice increment results in each protocol, sorted by scanner (different colors).

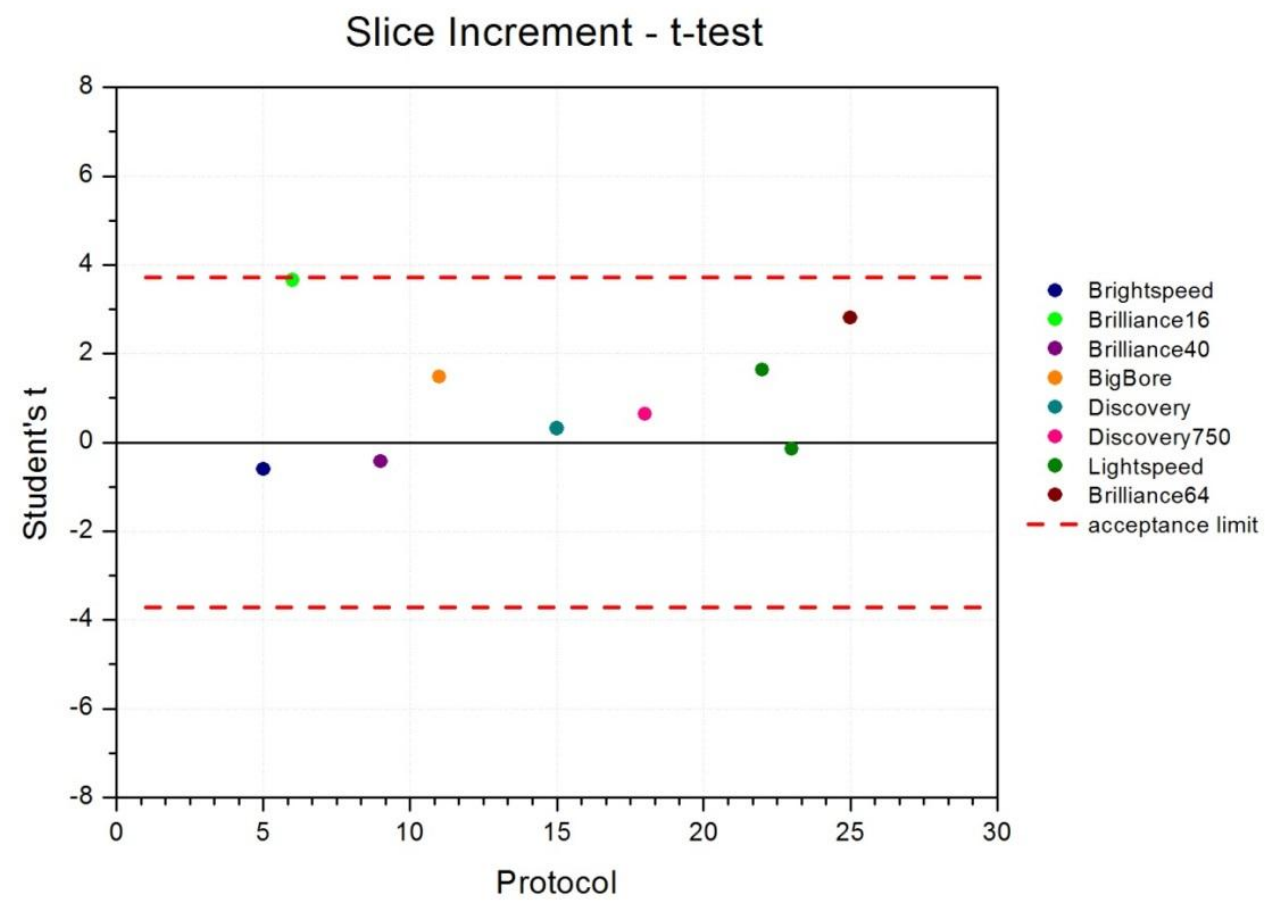

Figure 25: Student's $t$ value for slice increment for each protocol. The QC does not measure it at all protocols. The red dashed lines represent the acceptance interval. All values are within the acceptance interval. 


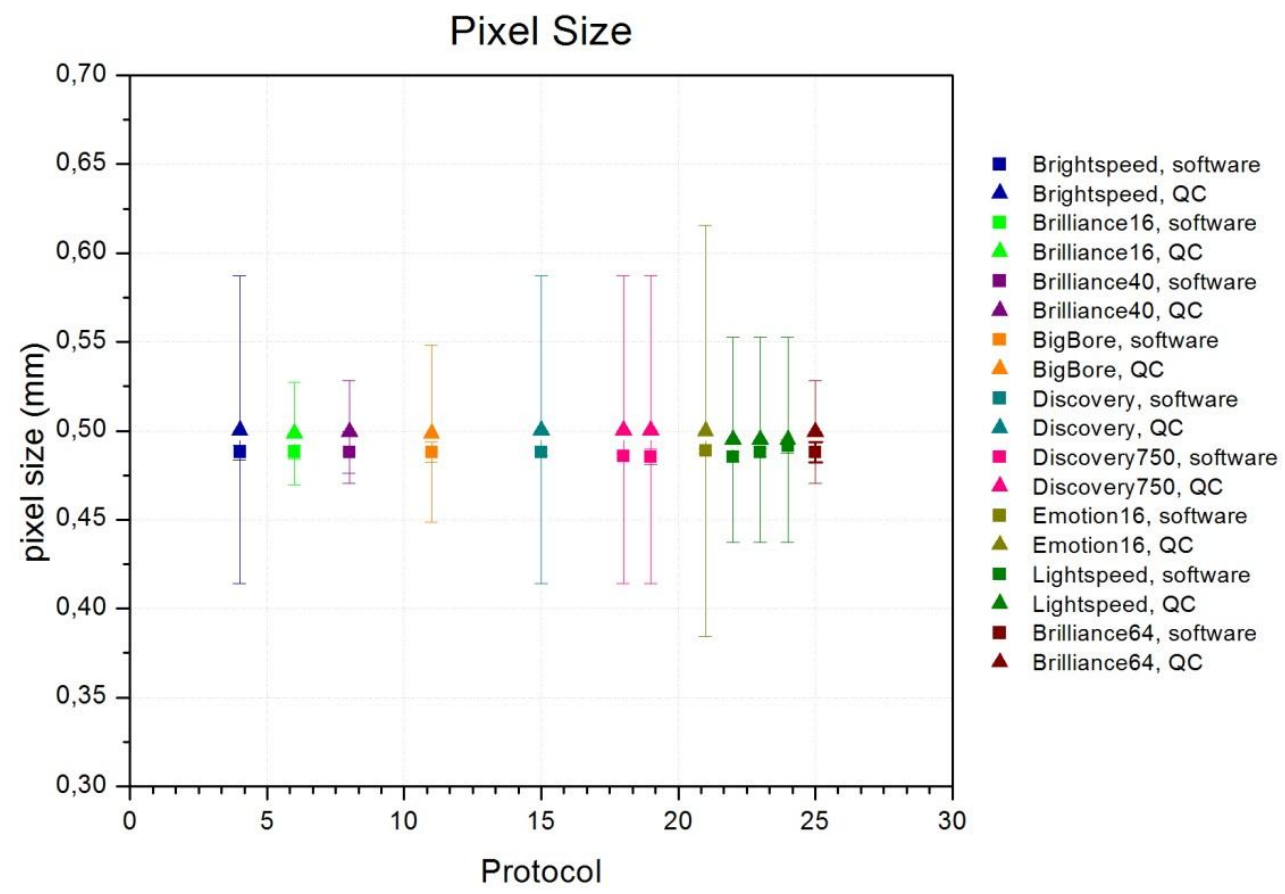

Figure 26: Pixel size obtained by the software (squares) and the QC (triangles) for each protocol, sorted by scanner (different colors).

\section{Pixel Size - percentual difference Software-QC}

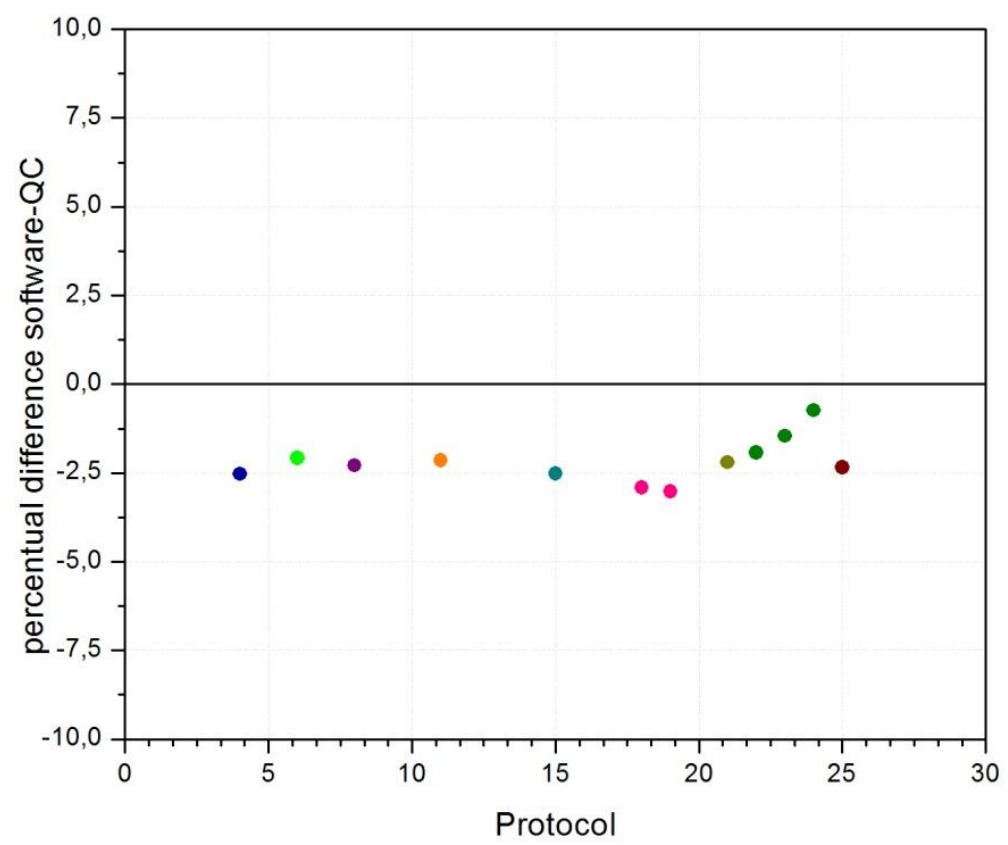

Brightspeed

Brilliance16

- Brilliance40

- BigBore

- Discovery

- Discovery 750

- Emotion16

- Lightspeed

- Brilliance64

Figure 27: Percentual difference between the software and the QC pixel size results in each protocol, sorted by scanner (different colors). 


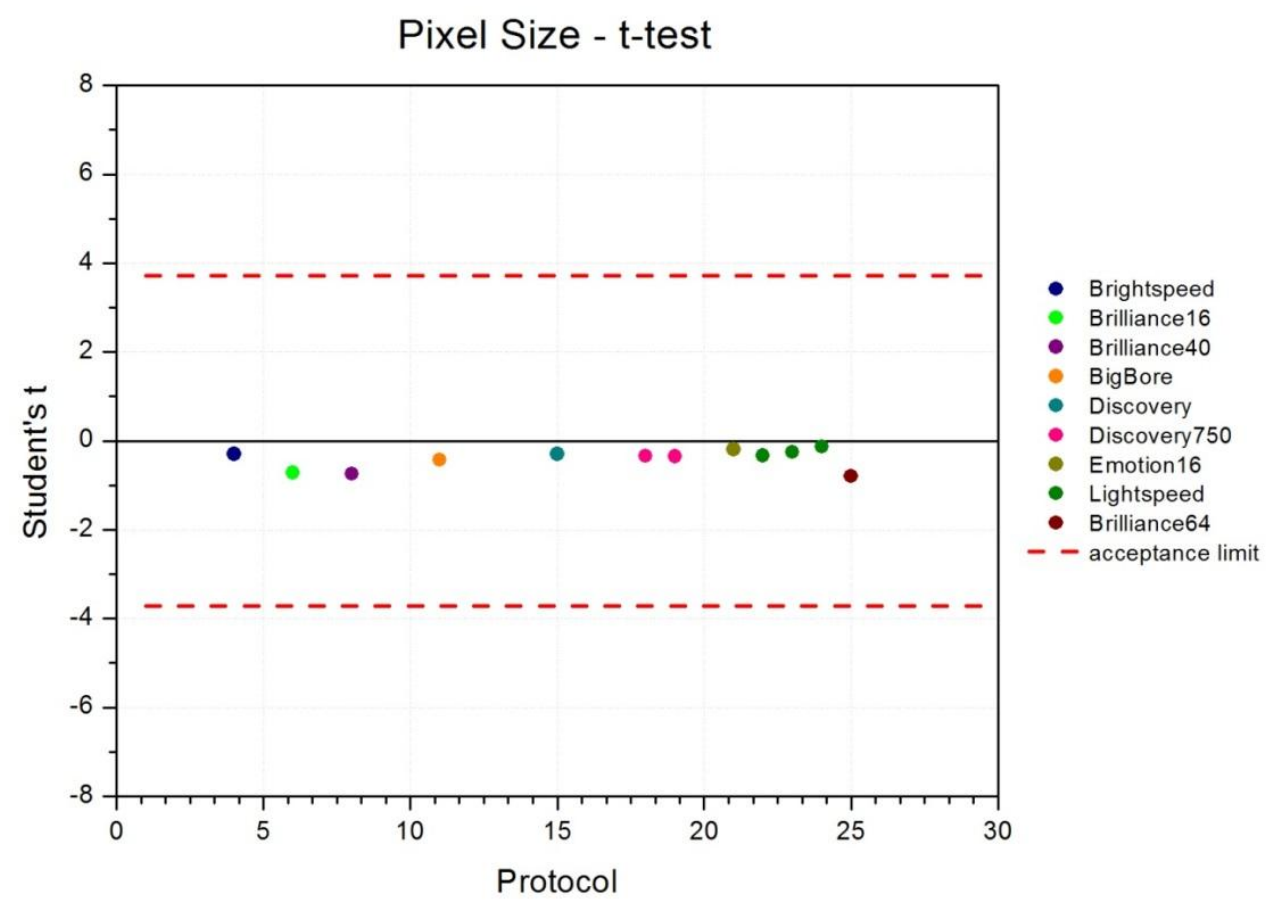

Figure 28: Student's $t$ value for pixel size. The QC does not measure it at all protocols. The red dashed lines represent the acceptance interval. All values are within the acceptance interval.

\section{CT number linearity evaluation - linear coefficient}

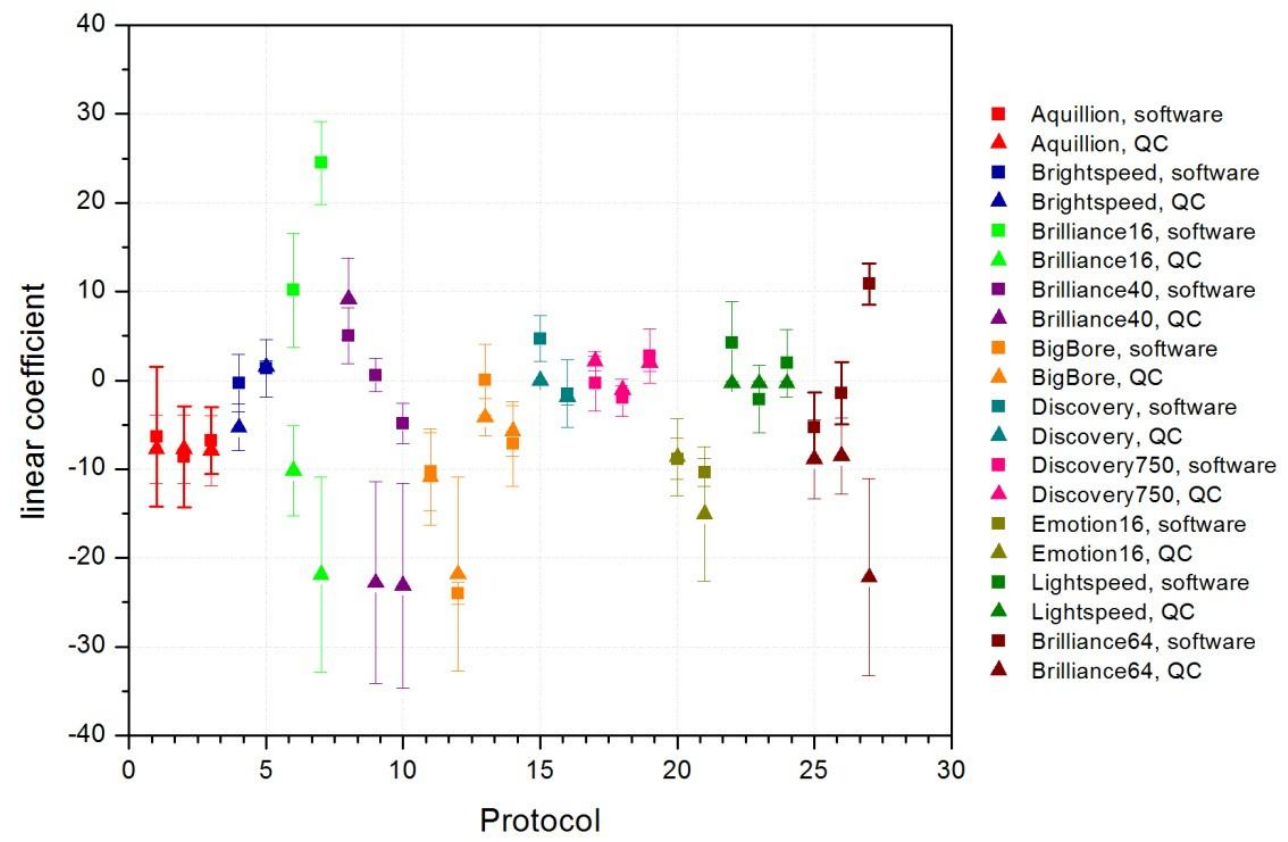

Figure 29: Linear coefficient of the CT number calibration obtained by the software (squares) and the QC (triangles) for each protocol, sorted by scanner (different colors). 
CT number linearity evaluation - linear coefficient t-test

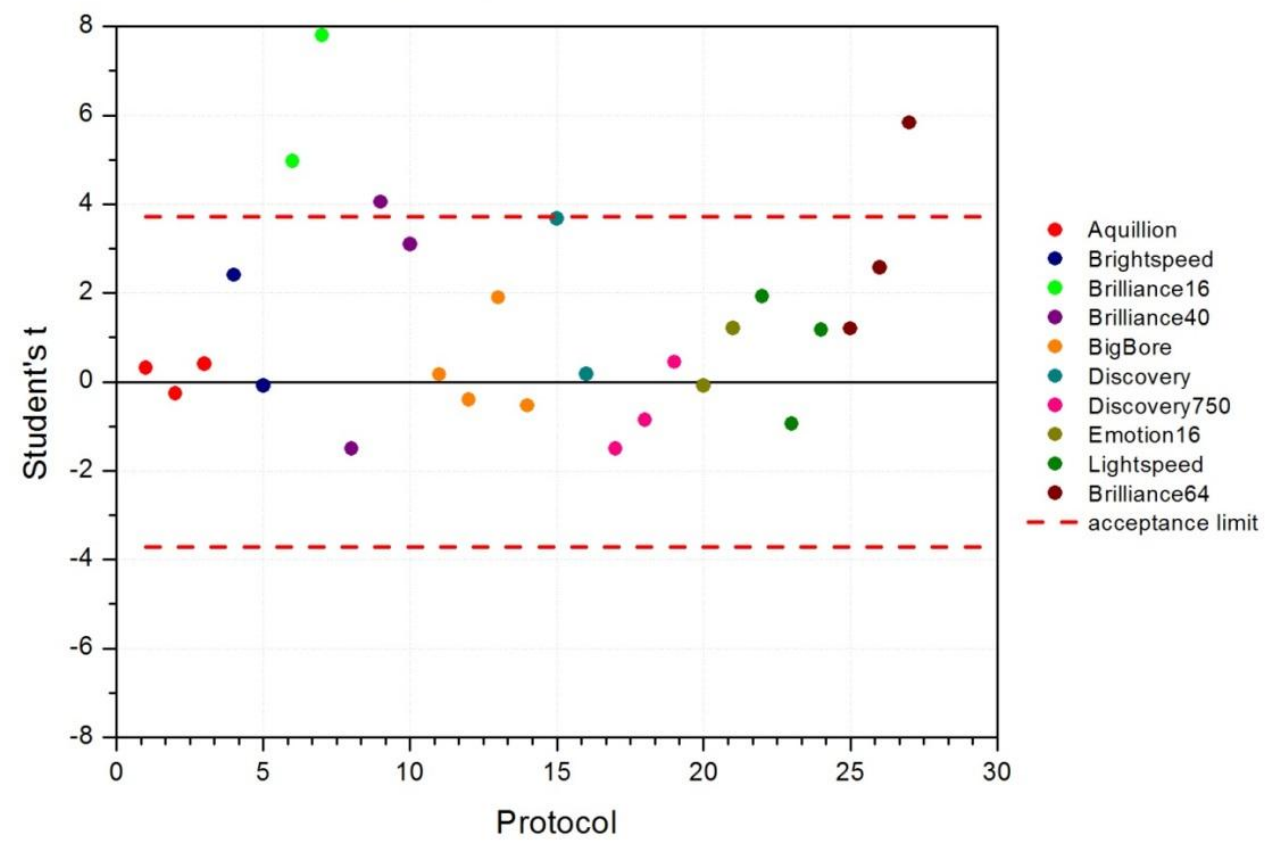

Figure 30: Student's $t$ value for the linear coefficient of the fit for CT number calibration. The red dashed lines represent the acceptance interval. Protocols 6, 7,9 and 27 are out of the interval.

CT number linearity evaluation - angular coefficient

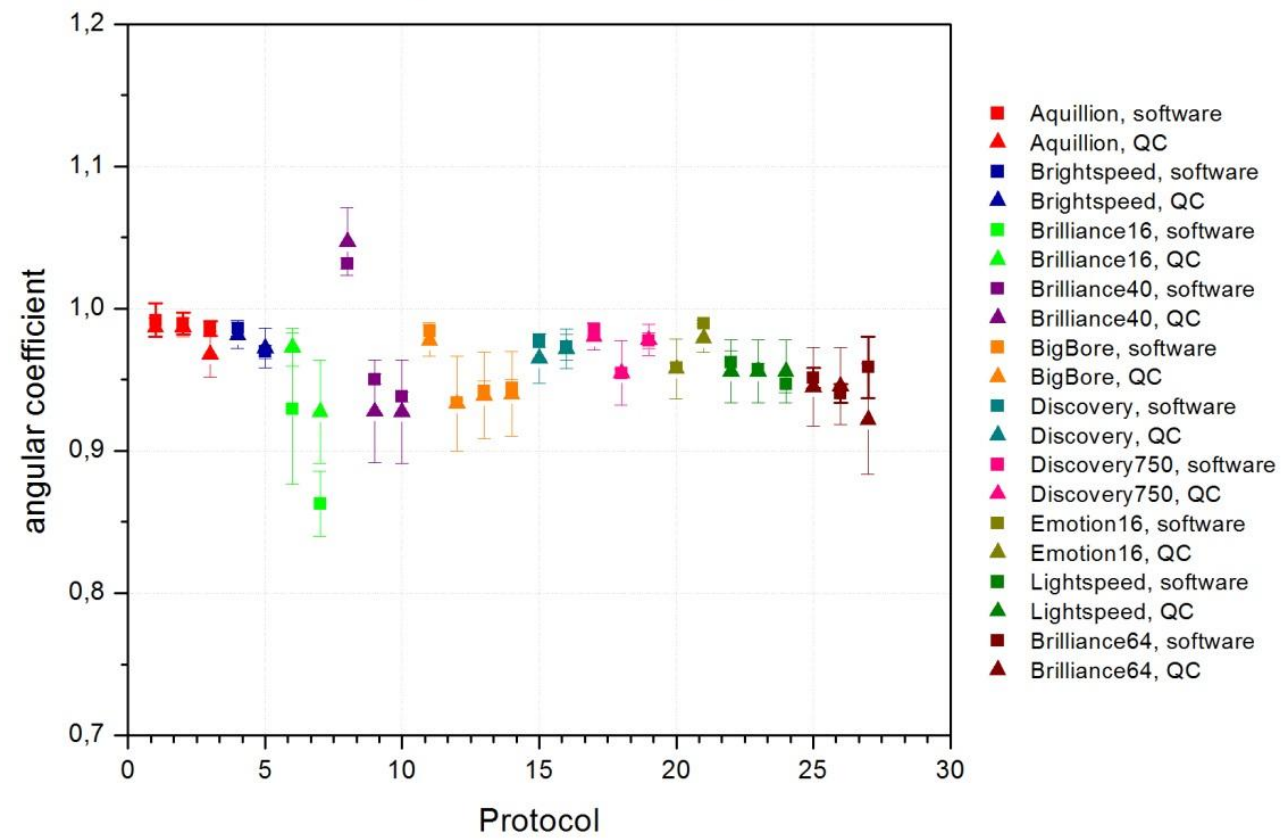

Figure 31: Angular coefficient of the CT number calibration obtained by the software (squares) and the QC (triangles) for each protocol, sorted by scanner (different colors). 


\section{CT number linearity evaluation - angular coefficient t-test}

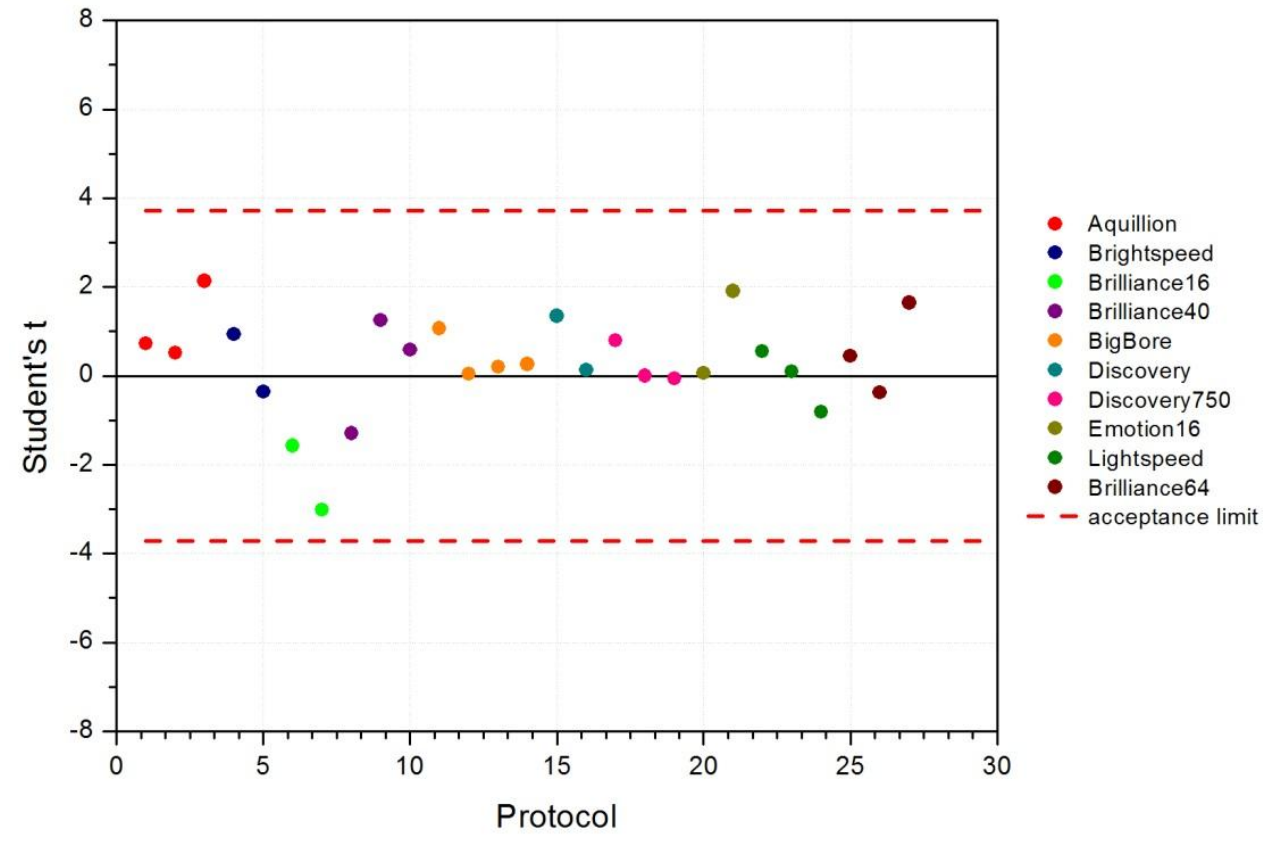

Figure 32: Student's $t$ value for the angular coefficient of the fit for CT number calibration. The red dashed lines represent the acceptance interval. All values are within the acceptance interval.

Figures 20 and 28 show that some $\mathrm{t}$ values are outside the acceptance range. These values were checked for possible methodology errors and, if none was found, were considered statistical errors (i.e., falsely rejected results), as discussed in Appendix B.

Figure 22 shows the slice thickness of protocol 19 is also outside the acceptance interval. No methodology error was found in either the software or the QC report, so it is a statistical error.

Figure 26 shows the uncertainties of the pixel size value from the QC report are much larger than those from the software. Figure 28 shows the $t$ values obtained for pixel size are always very close to zero. It indicates that the pixel size uncertainties from the QC report are likely to be overestimated.

Figure 30 shows that the software results for the linear coefficient at 4 protocols $(6,7,9$ and 27) were not compatible with the QC results. It was found that QC uses the Excel built-in fitting routine, which does not take into account the uncertainty of each point. When these particular fits were recalculated, using the same data points at the QC report $\left(C T_{\text {exp }}, \overline{C T}, \sigma_{C T}\right)$, the $t$ values of 3 out of these 4 protocols got within the acceptance interval $(-2.92,0.14,1.94$ and 3.90 respectively). 
In the other protocols, the QC also used the Excel spreadsheet calculation, so their fitted values also do not consider the uncertainty of the data points. Even though, they were within the range $|t|<3.71$. If these values were all recalculated the tendency is that the $t$ values get closer to zero, so it was not considered necessary to recalculate all.

So, these 4 protocols with unacceptable $t$ values were not considered a software inconsistency: 3 of them come from the choice of fitting algorithm at the QC program, and one can be considered a statistical error.

Among all these graphs there are a total of $101 t$ values, and only 2 values had a $t$ outside the acceptance interval. This is close to the expected behavior. As discussed in section 3.4.1, given the chosen $p=0.01$, it is expected to have around 1 in a 100 rejected values. The probability of this particular result is $18.7 \%$ (binomial distribution with $p=0.01,101$ "attempts" and 2 "successes"), so it is not an unlikely result. It can be concluded that the software results are in good agreement to the results produced by the QC normal routine procedures.

\subsubsection{Noise Magnitude}

Figure 33 presents the noise values obtained by the software and the QC. Figure 34 shows the $F$ values calculated for the noise magnitude comparison, and the acceptance interval (red dashed lines) [0.934, 1.066].

Protocol 16 had a value much lower than expected (0.47), and was checked for mistakes. The QC report was reviewed and the noise magnitude recalculated. After this operation, the $\sigma$ value obtained was found to be compatible with the software result $(\sigma=5.58 H U ; F=1.02)$.

Protocol 15 was also out of the acceptance interval, but close to it. It was also checked, but no mistake was found. From the $25 F$ values calculated (not accounting protocol 16 ), only this one is outside the interval; the probability of this particular result is $36.5 \%$ (binomial distribution with $p=0.05,25$ "attempts" and 1 "success"). So, it can be said that the noise estimation done by the software is in agreement with the QC result. 


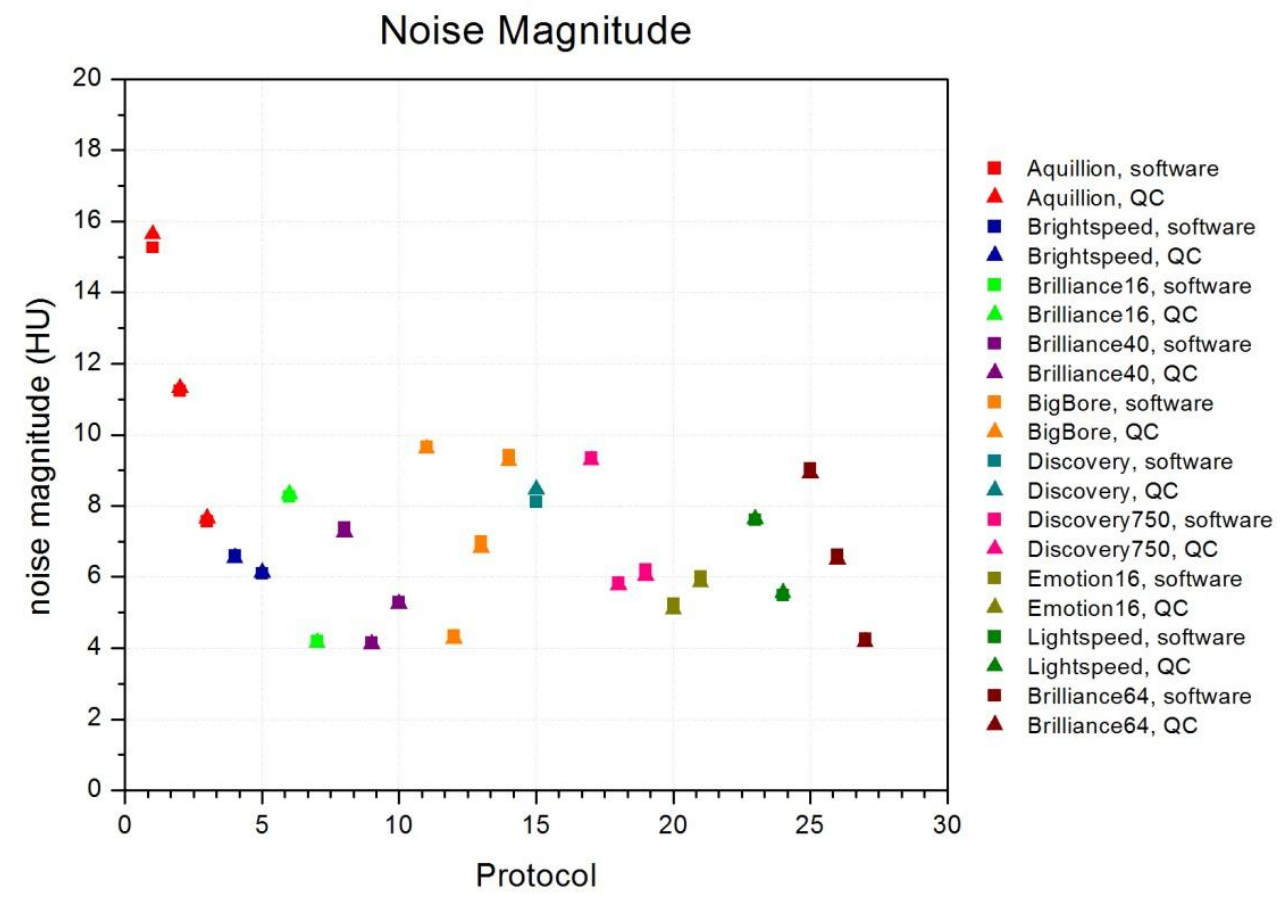

Figure 33: Noise magnitude obtained by the software (squares) and the QC (triangles) for each protocol, sorted by scanner (different colors).

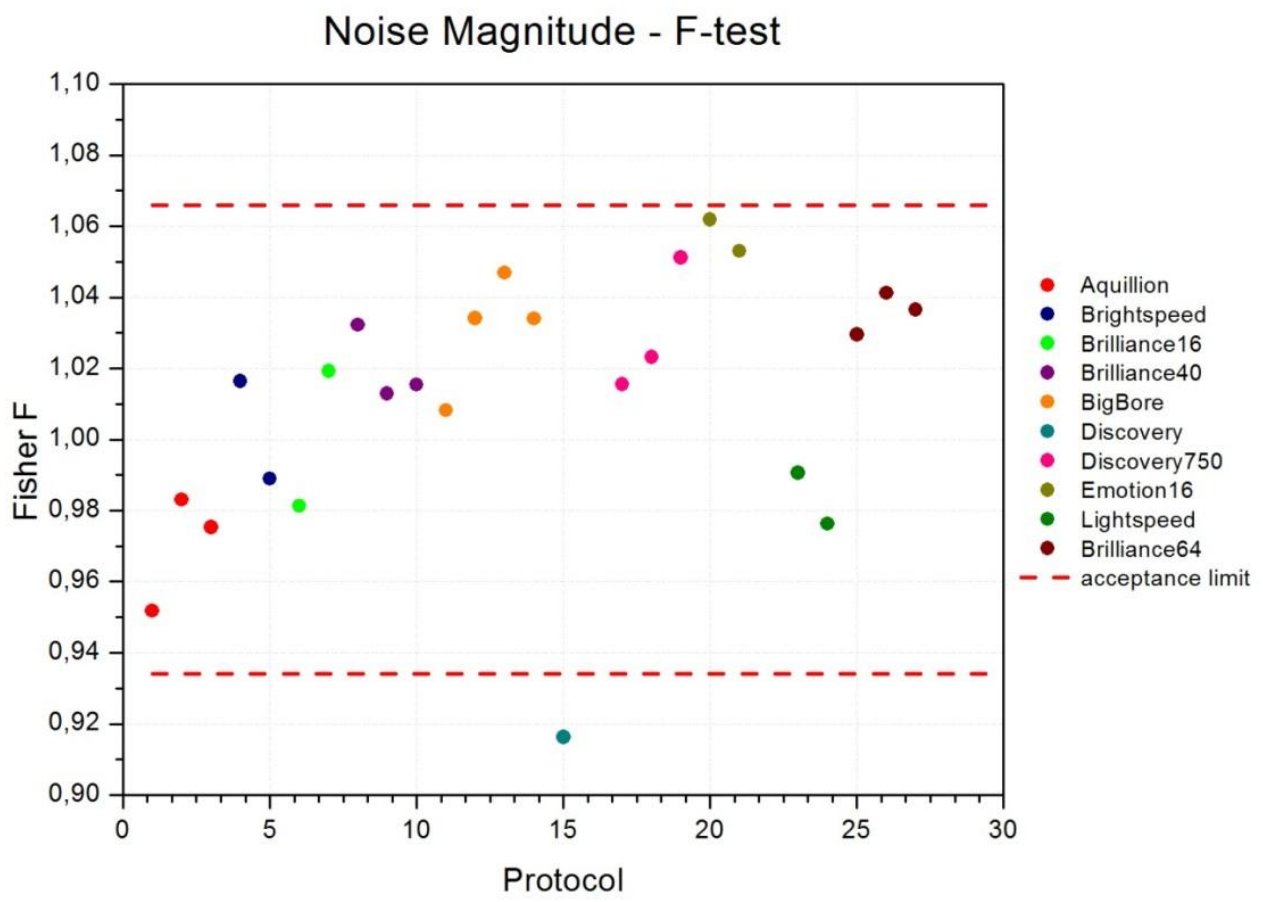

Figure 34: Fisher $F$ value comparing the noise magnitude of the software and the QC report, sorted by scanner (different colors). The red dashed lines represent the acceptance interval. Protocols 15 and 16 are outside the acceptance interval (16 is not represented, it is 0.47 , much below the scale of this graphic). 


\subsubsection{Cutoff Frequency}

Figure 35 shows all the 3 estimated frequencies $\left(f_{c}, f_{10 \%}\right.$ and $\left.f_{Q C}\right)$ for each protocol, in units of pixel $^{-1}$. The uncertainties of $f_{c}$, as expected, are difficult to be estimated accurately and varied in a very broad range, from 0.0023 to 0.15 pixel $^{-1}$. This is a consequence of interpolating a value in an interval where the function derivate is close to zero. Given two points, $A=\left(x_{A}, y_{A}\right)$ and $B=\left(x_{B}, y_{B}\right)$, for a fixied $y=y_{m}$ the interpolated value $x_{m}$ is:

$$
x_{m}=\frac{y_{m}-y_{A}}{a}+x_{A}
$$

Where $a$ is the angular coefficient of the line from $A$ to $B$. This angular coefficient appears in the denominator, therefore in the uncertainty calculation it also appears on the denominator. So, the smaller the inclination of this line is, the larger the uncertainty of the interpolated $x_{m}$ value. This is why, for some protocols, the uncertainty of $f_{c}$ is so large and in other protocols it is not: this uncertainty depends very strongly on the angular coefficient between the two points where the interpolation is done.

The values of $f_{Q C}$ vary discretely (example: there are protocols where $f_{Q C}$ is $0.33,0.37$ and 0.40 , but there are no values between these). This is due to the process that estimates $f_{Q C}$ : the phantom has bar patterns at specific spatial frequencies, so the estimated values of $f_{Q C}$ are also discrete.

It was observed that the QC frequency values are almost always between $f_{10 \%}$ and $f_{c}$, and almost always close to $f_{10 \%}$. In only 3 protocols $f_{Q C}$ is lower than $f_{10 \%}$ (protocols 11,12 and 18), and in only one protocol $f_{Q C}$ and $f_{10 \%}$ are very different from each other (more than 3 uncertainties apart, in protocol 27). This is a good indicator that the software results for $f_{10 \%}$ are consistent with the $\mathrm{QC}$ results, $f_{Q C}$.

The fact that $f_{c}$ is always above $f_{Q C}$ is consistent with the expected behavior (the cutoff frequency of a system plus the human vision should always be lower than the system's cutoff frequency).

The frequency at $10 \%$ of the MTF obtained by the software in some protocols was compared to values found in the literature. Toshiba Aquilion user manual ${ }^{40}$ states that its $f_{10 \%}$ value is $0.78 \mathrm{lp} / \mathrm{mm}$ for the FC70 filter. The value obtained by the software in protocol 2 with this same scanner and filter (converted to $l p / m m$ using the pixel size), 
was $0.784(5) \mathrm{lp} / \mathrm{mm}$. For the GE Lightspeed scanner with the standard head filter (protocol 22), McCann and Alasti ${ }^{81}$ report $f_{10 \%}$ of $0.64 \mathrm{lp} / \mathrm{mm}$ (no uncertainty provided), and the software obtained $0.6611(13) \mathrm{lp} / \mathrm{mm}$. These results indicate that the software results are in agreement with other authors, even if those utilized different MTF measurement methods. Other scanner evaluations utilizing the $f_{10 \%}$ value were found for the GE Discovery $750 \mathrm{HD}$ and Siemens Somatom, but since they did not use the same filters, the comparison is inadequate ${ }^{81 ; 82}$.

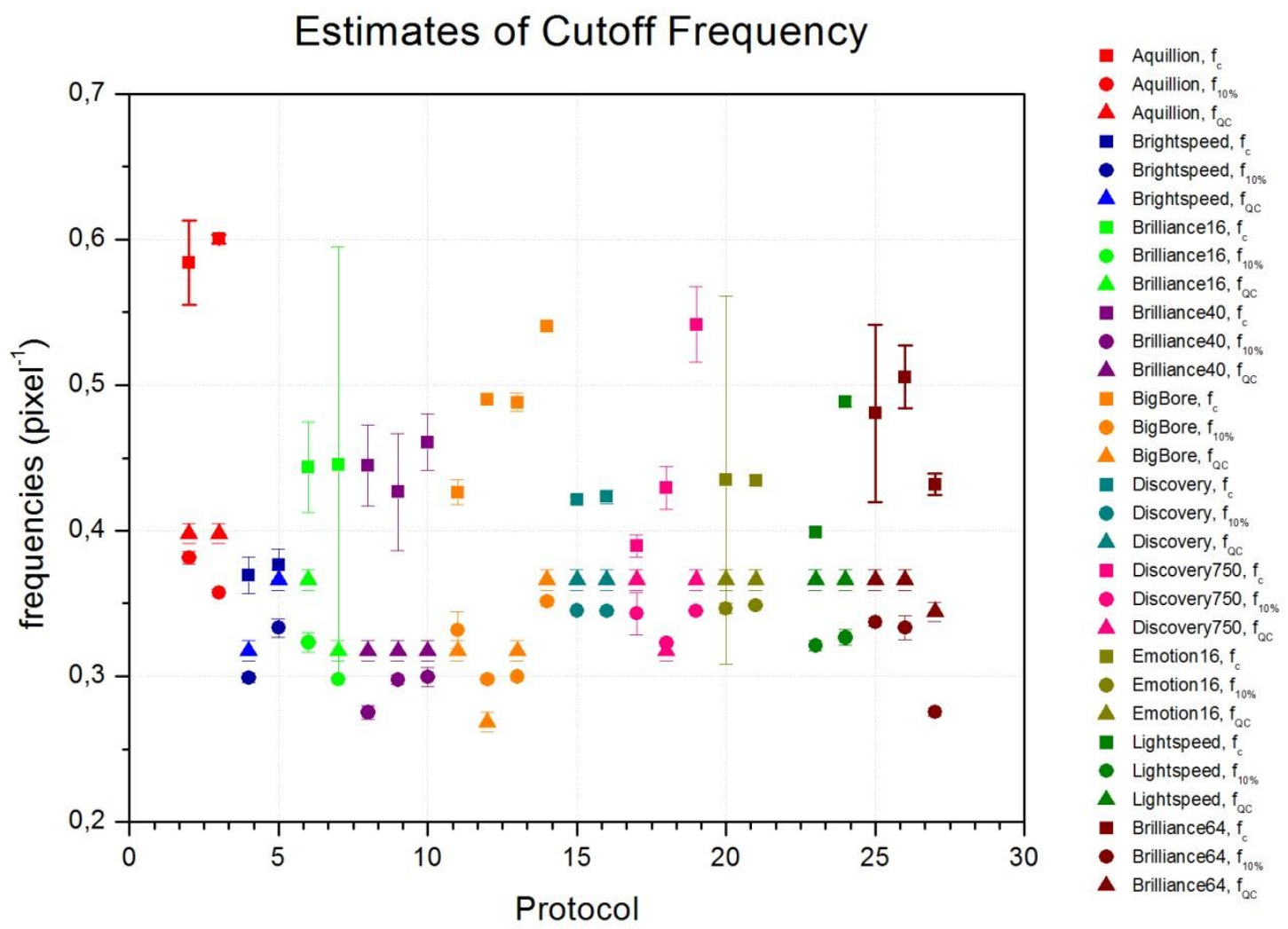

Figure 35: cutoff frequency estimated by the software (squares, $f_{c}$ ), frequency at $10 \%$ of the $M T F$ (circles, $f_{10 \%}$ ) and the cutoff frequency estimated by the QC (triangles, $f_{Q C}$ ) for each protocol, sorted by scanner (different colors).

\subsection{4. $M T F$ and NPS}

Figures 34 to 46 show the obtained MTF (upper plot, in red), obtained NPS (lower plot, red solid line) and the NPS calculated from the MTF (lower plot, blue points with uncertainty bars). They also show the coefficients fitted with equation (86) and the calculated $Q_{\text {red }}$. 
The software also saves the 2-dimensional NPS function, but it is not used for the validation. These are presented in the Appendix D for completeness only, as the 2D NPS does not present any information that the radial NPS does not also present. 
(a)

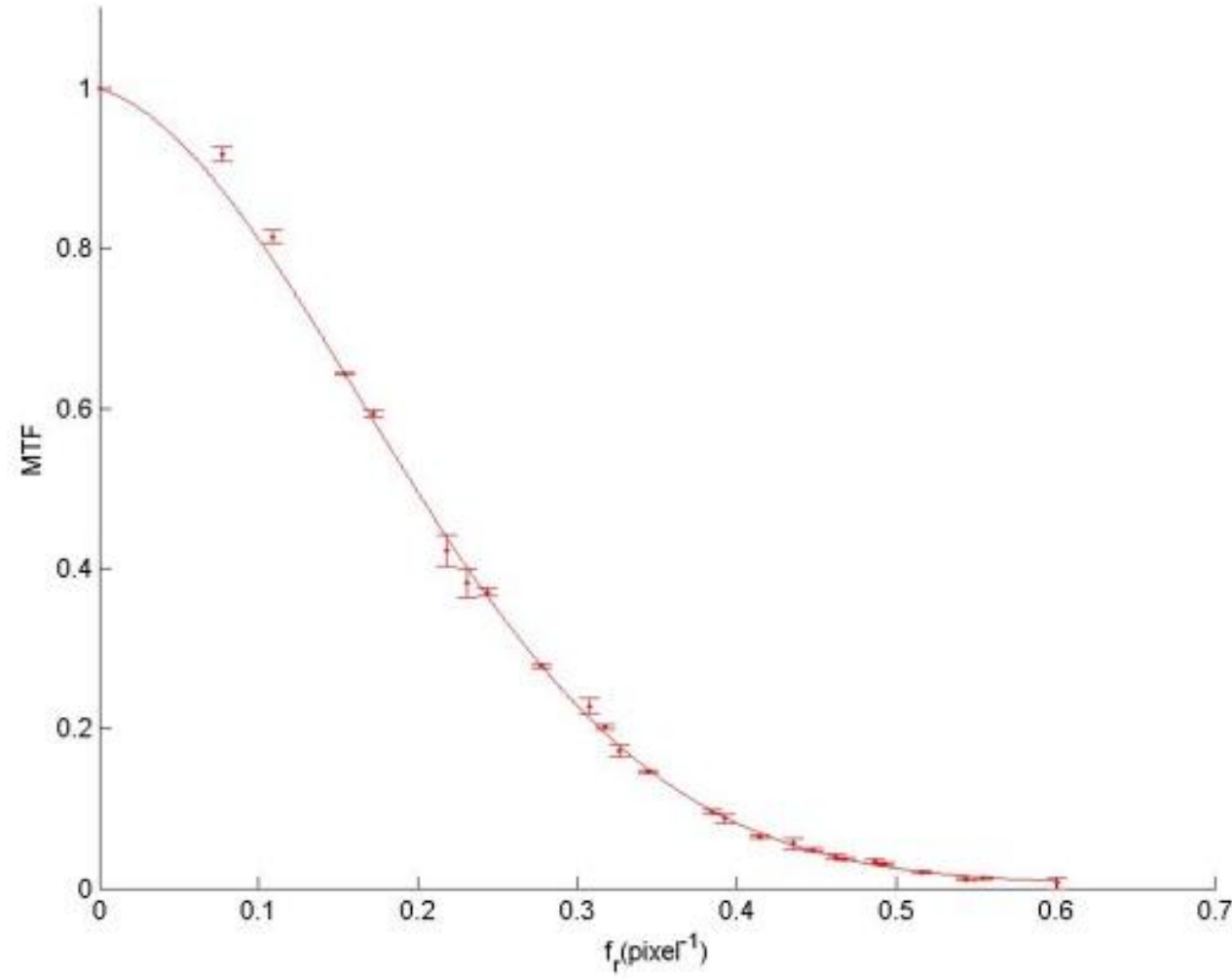

(b)

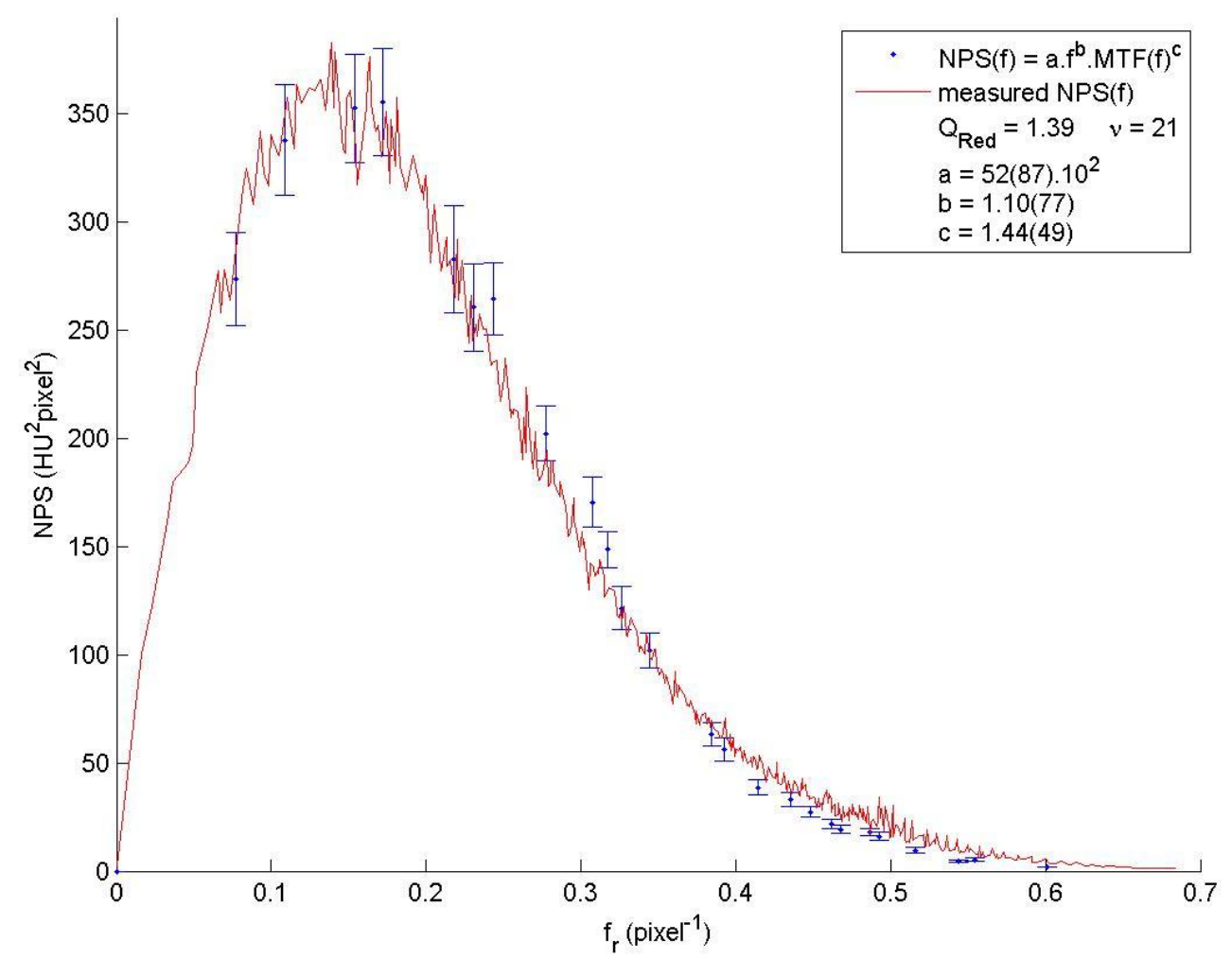

Figure 36: (a) MTF fitted by an illustrative Gaussian curve. (b) NPS measured at protocol 2 (Toshiba Aquillion, axial mode, $1.0 \mathrm{~mm}$ slice thickness, $240 \mathrm{~mm}$ FOV) fitted as a function of the MTF. 
(a)

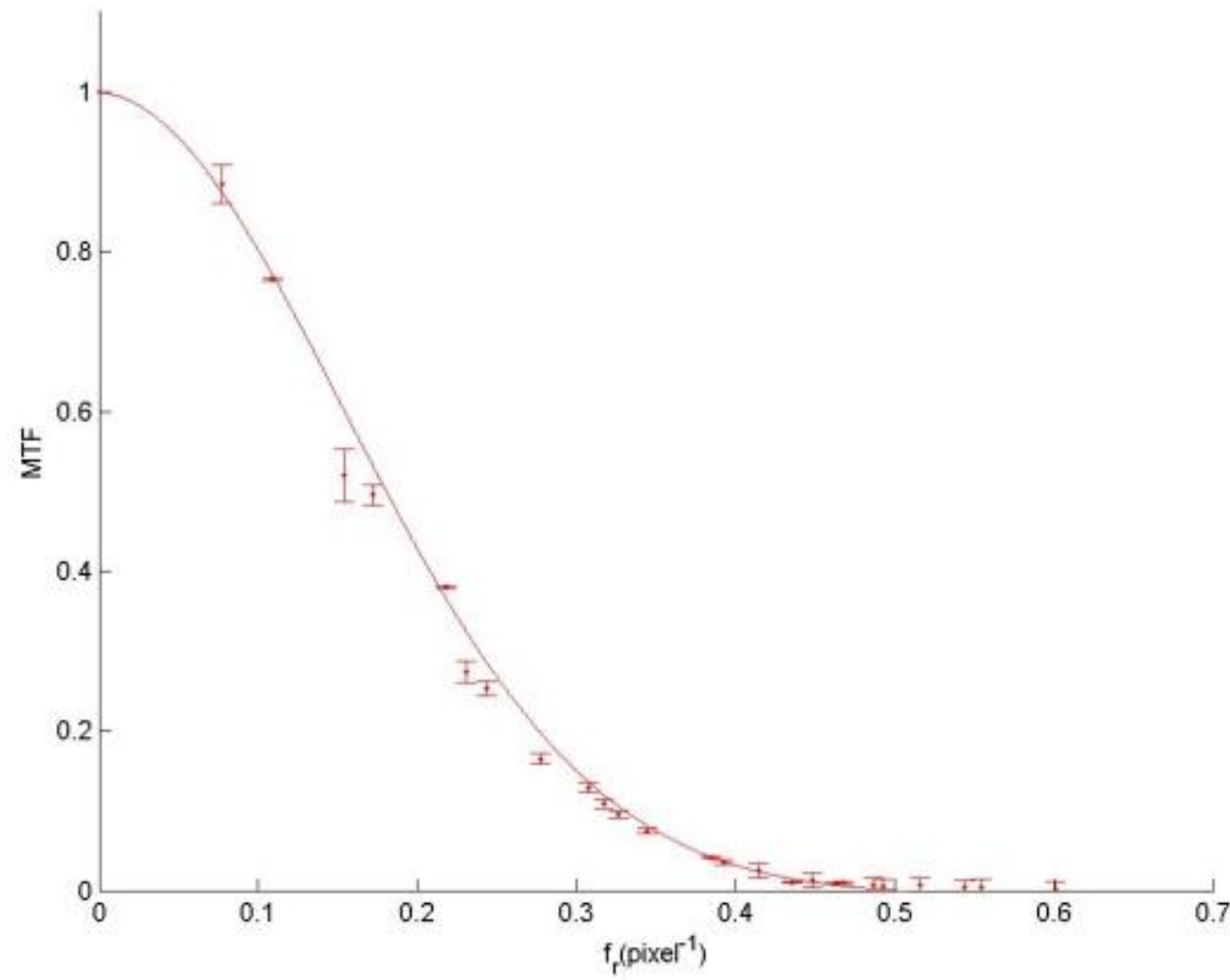

(b)

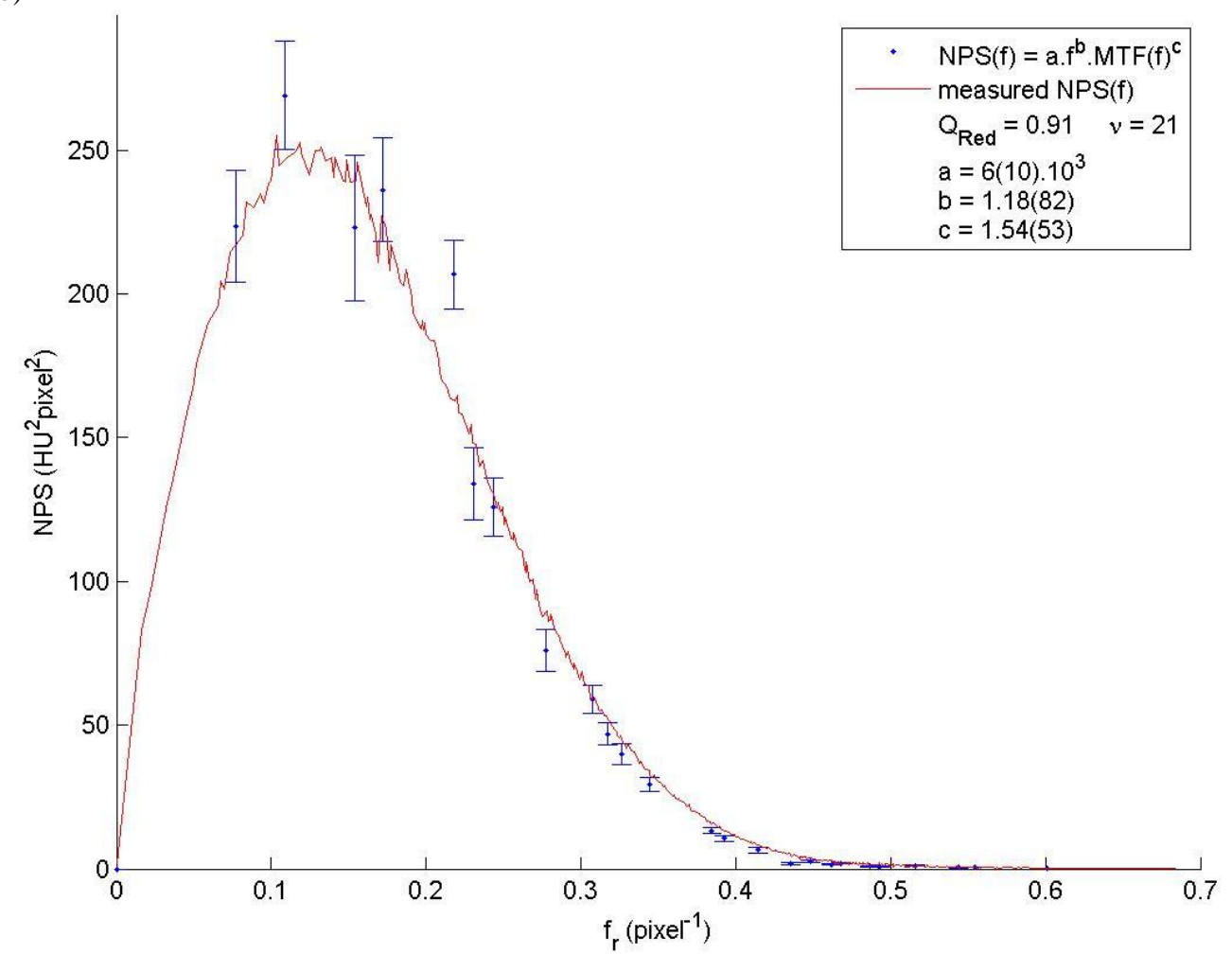

Figure 37: (a) MTF fitted by an illustrative Gaussian curve. (b) NPS measured at protocol 6 (Phillips Brilliance16, axial mode, $1.5 \mathrm{~mm}$ slice thickness, $250 \mathrm{~mm}$ FOV) fitted as a function of the MTF. 
(a)

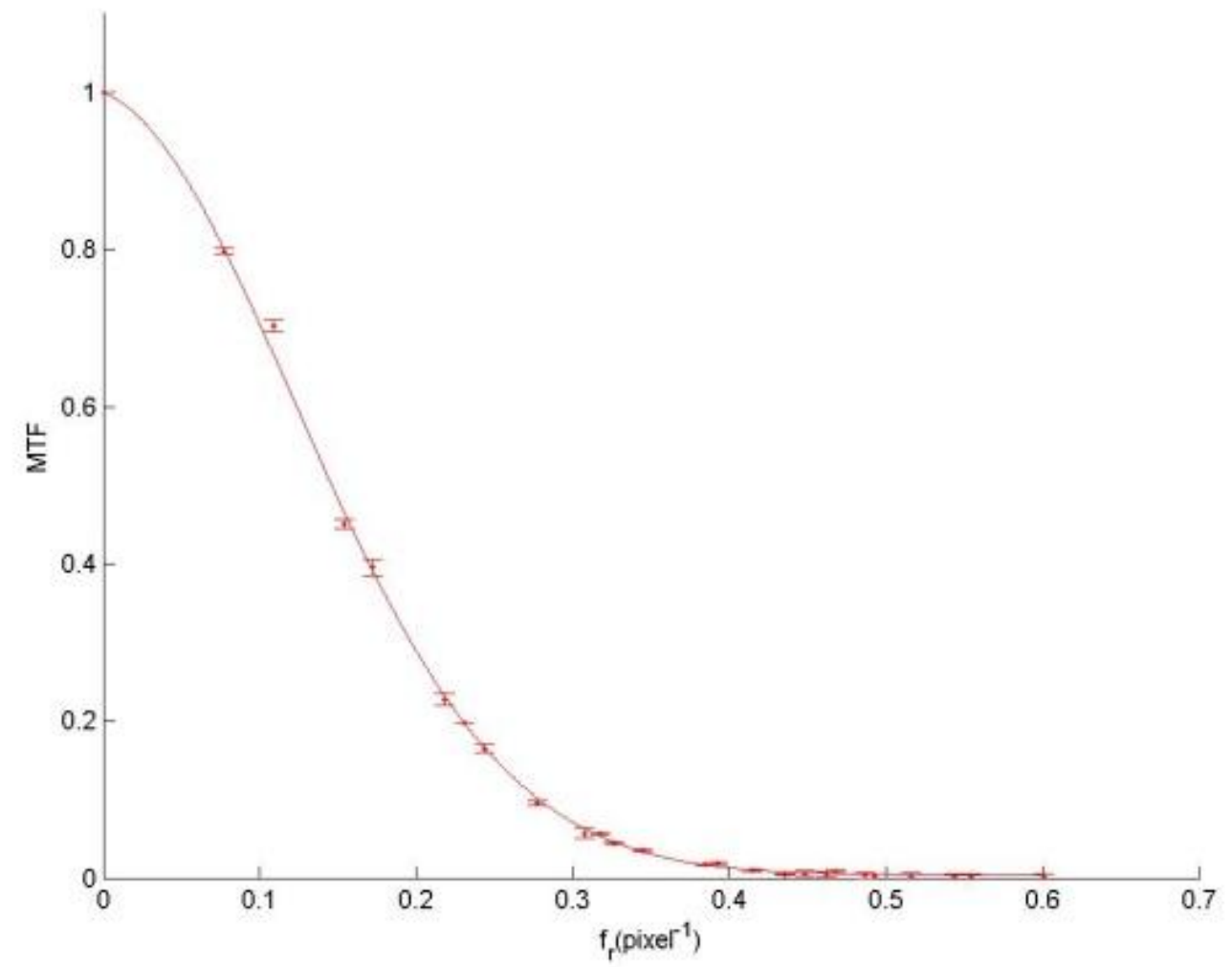

(b)

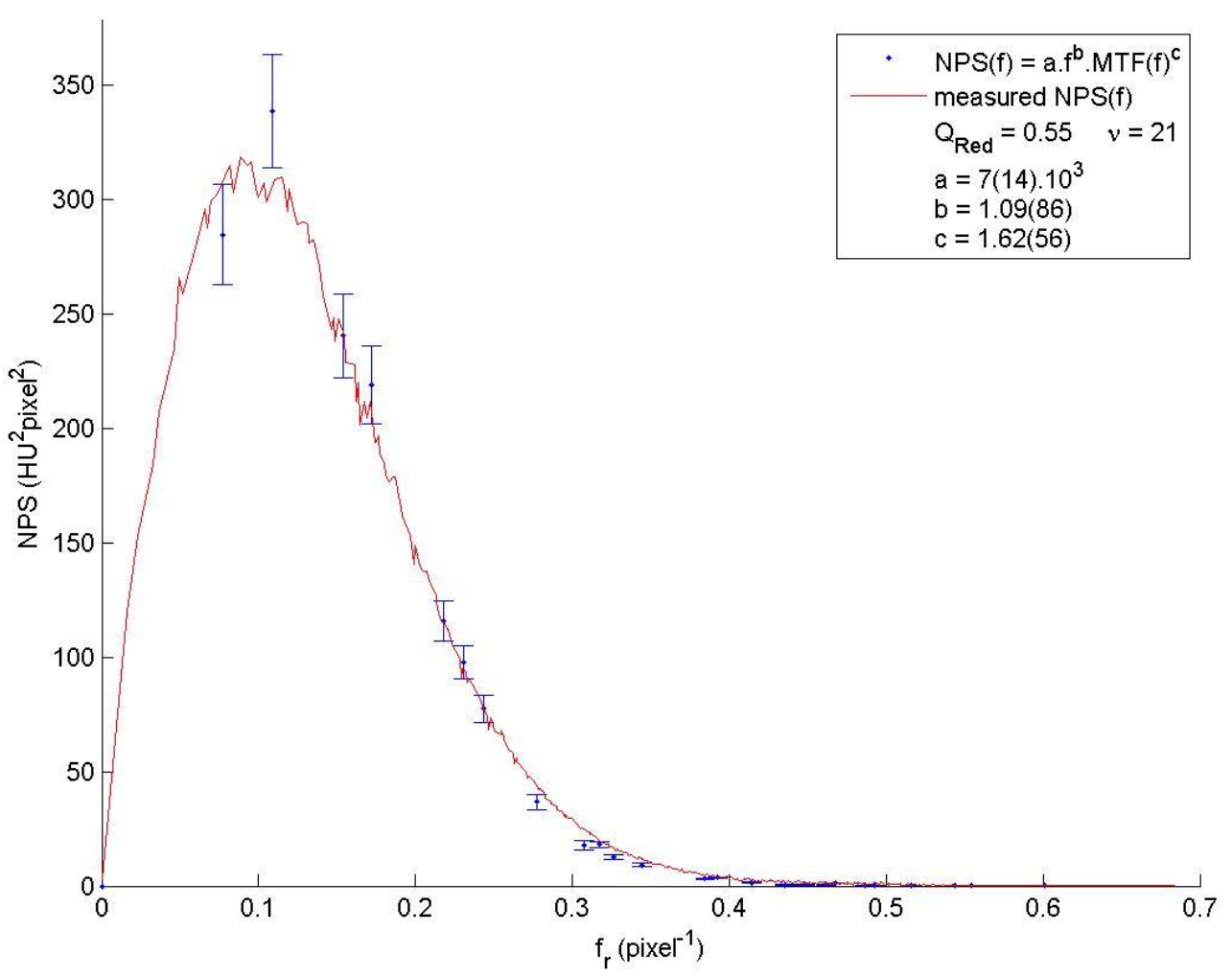

Figure 38: (a) MTF fitted by an illustrative Gaussian curve. (b) NPS measured at protocol 8 (Phillips Brilliance40, axial mode, $1.25 \mathrm{~mm}$ slice thickness, $250 \mathrm{~mm}$ FOV) fitted as a function of the MTF. 
(a)

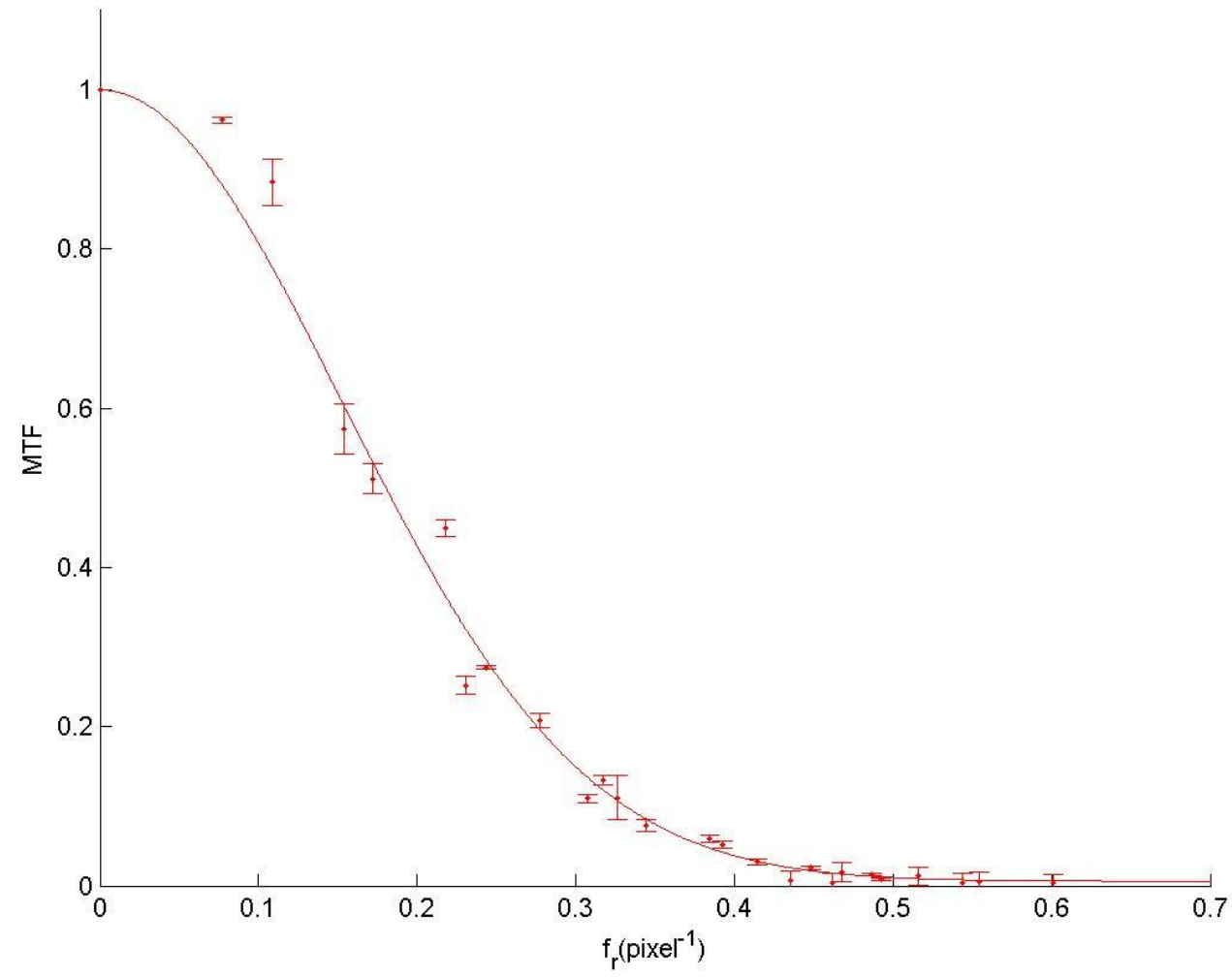

(b)

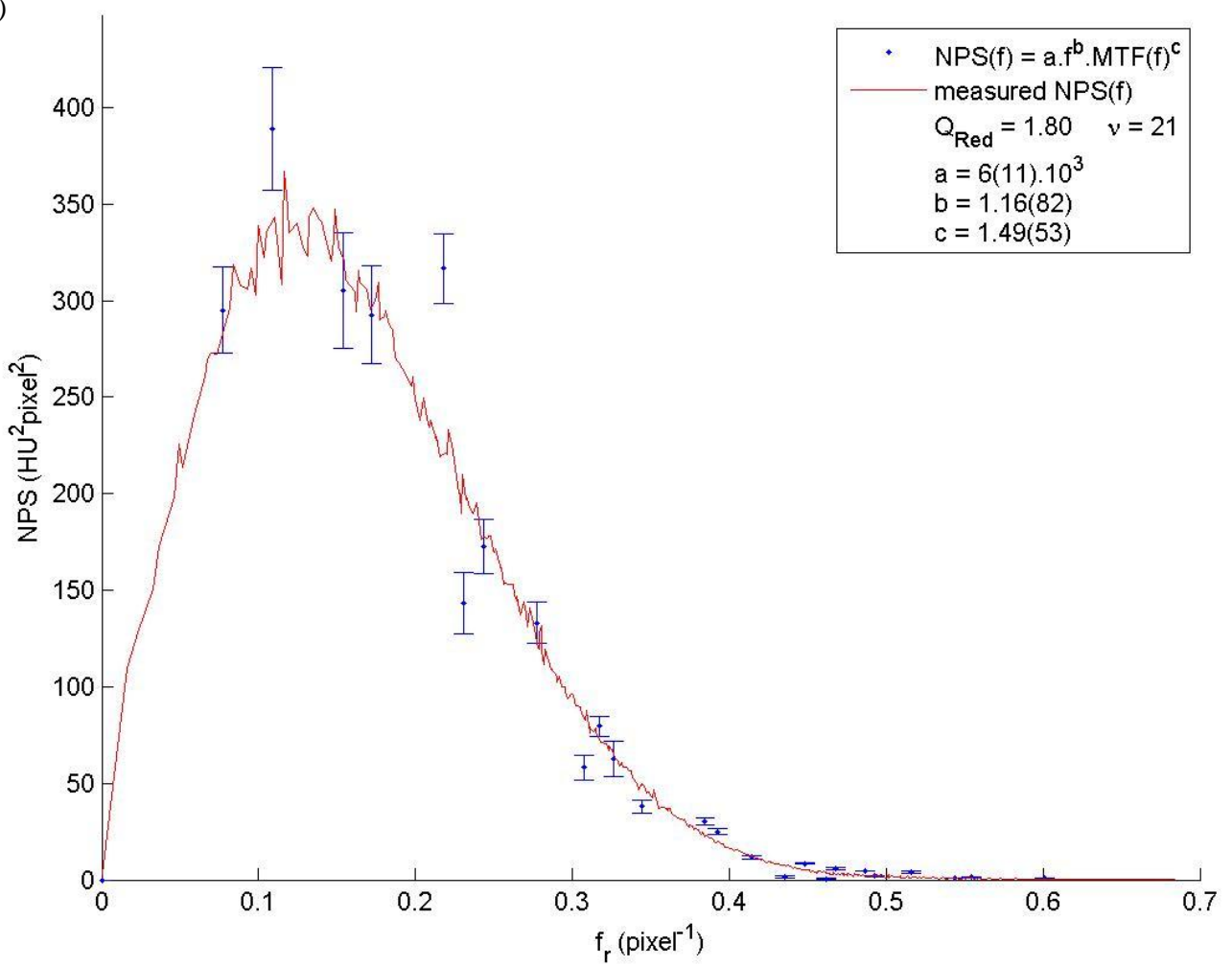

Figure 39: (a) MTF fitted by an illustrative Gaussian curve. (b) NPS measured at protocol 11 (Phillips Brilliance BigBore, axial mode, $1.5 \mathrm{~mm}$ slice thickness, $250 \mathrm{~mm}$ FOV) fitted as a function of the MTF. 
(a)

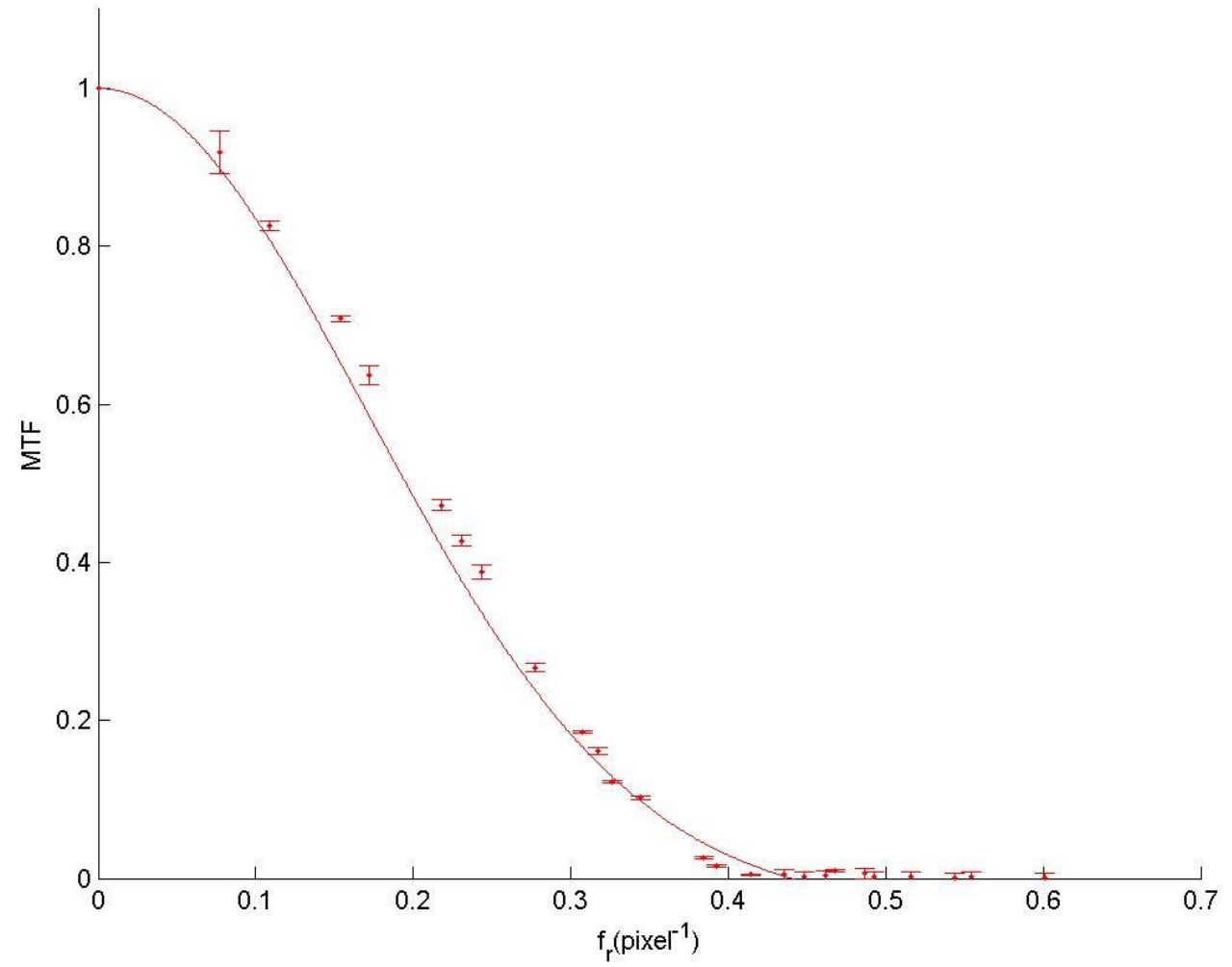

(b)

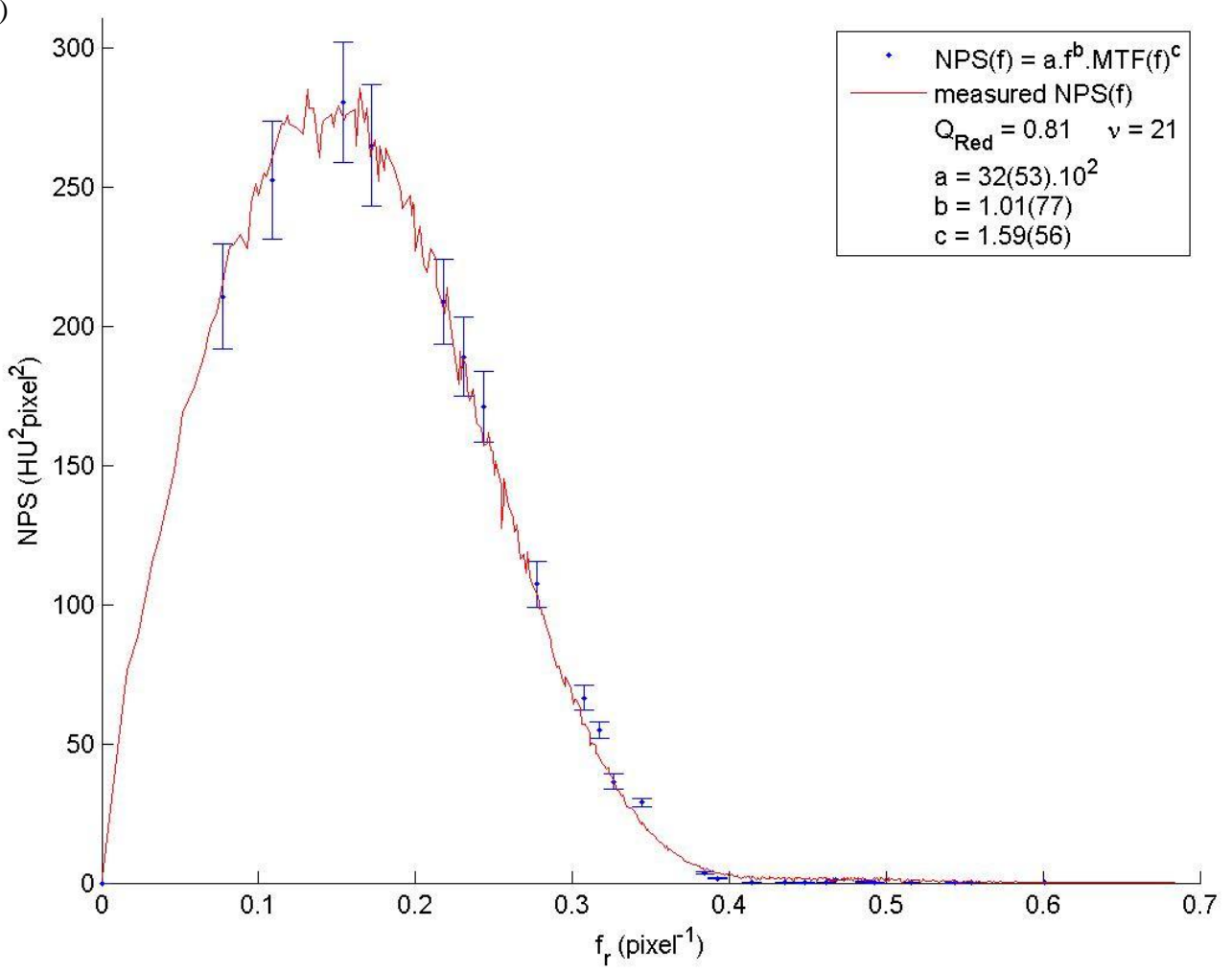

Figure 40: (a) MTF fitted by an illustrative Gaussian curve. (b) NPS measured at protocol 15 (GE Discovery690, axial mode, $1.25 \mathrm{~mm}$ slice thickness, $250 \mathrm{~mm}$ FOV) fitted as a function of the MTF. 
(a)

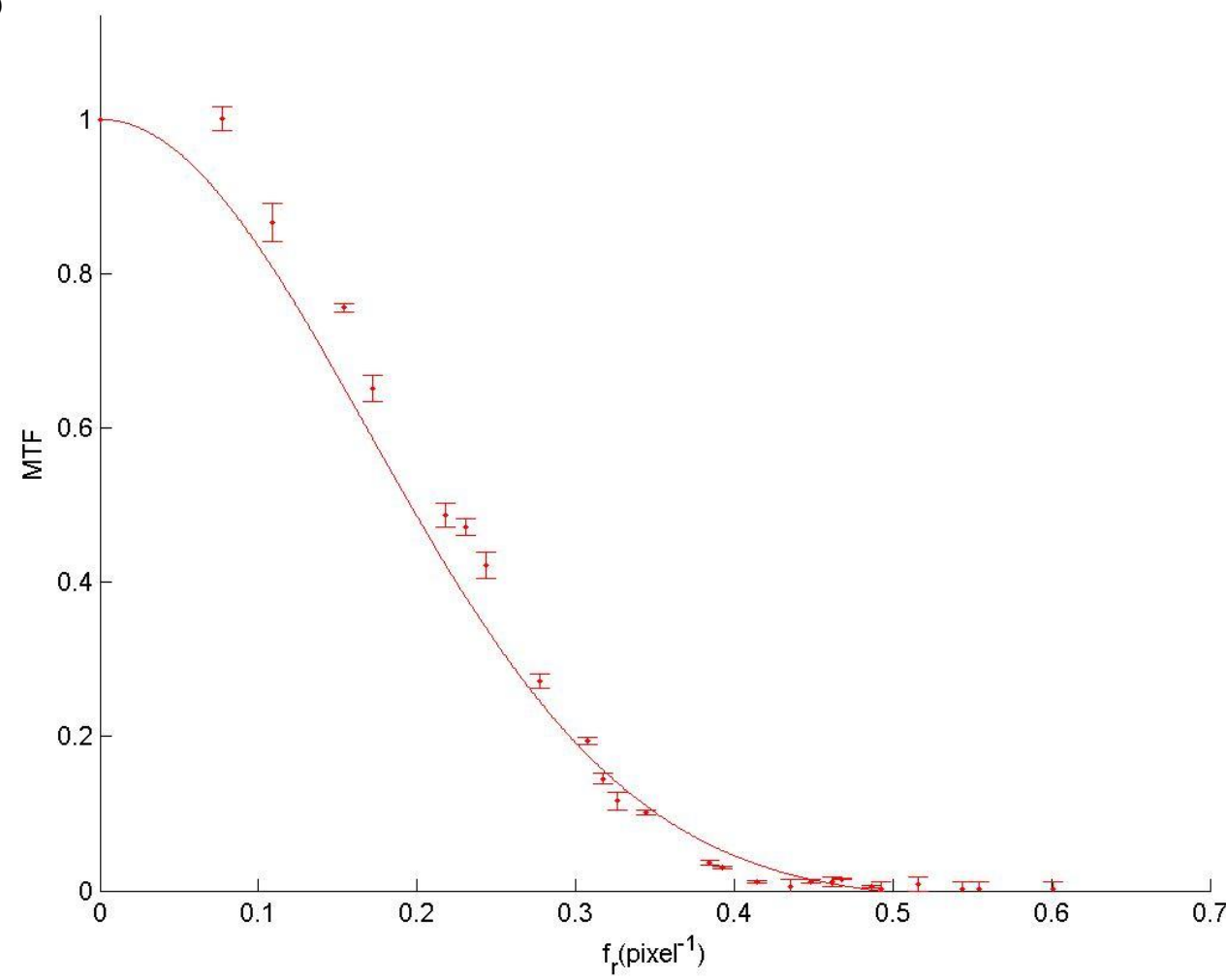

(b)

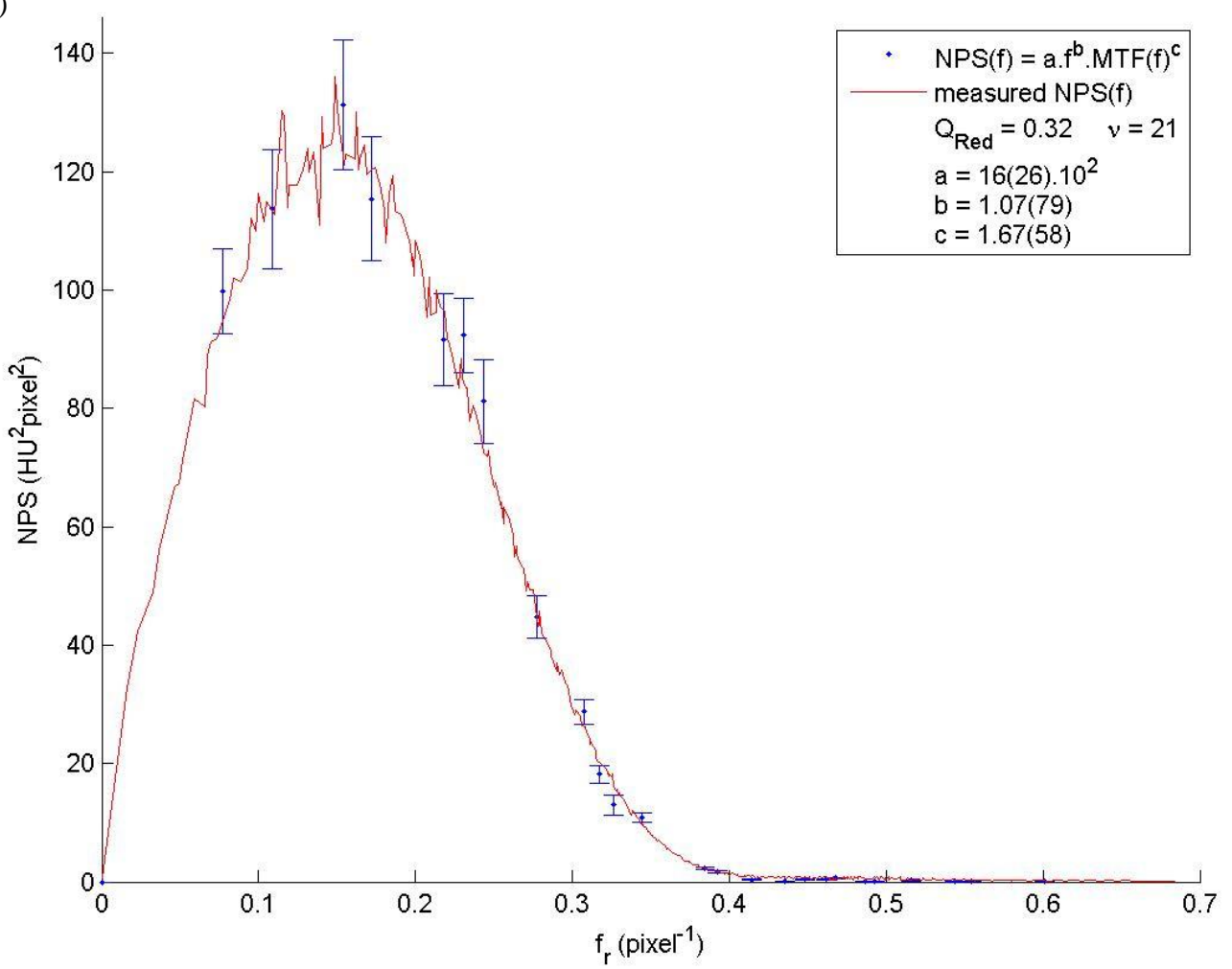

Figure 41: (a) MTF fitted by an illustrative Gaussian curve. (b) NPS measured at protocol 16 (GE Discovery690, axial mode, $2.5 \mathrm{~mm}$ slice thickness, $250 \mathrm{~mm}$ FOV) fitted as a function of the MTF. 
(a)

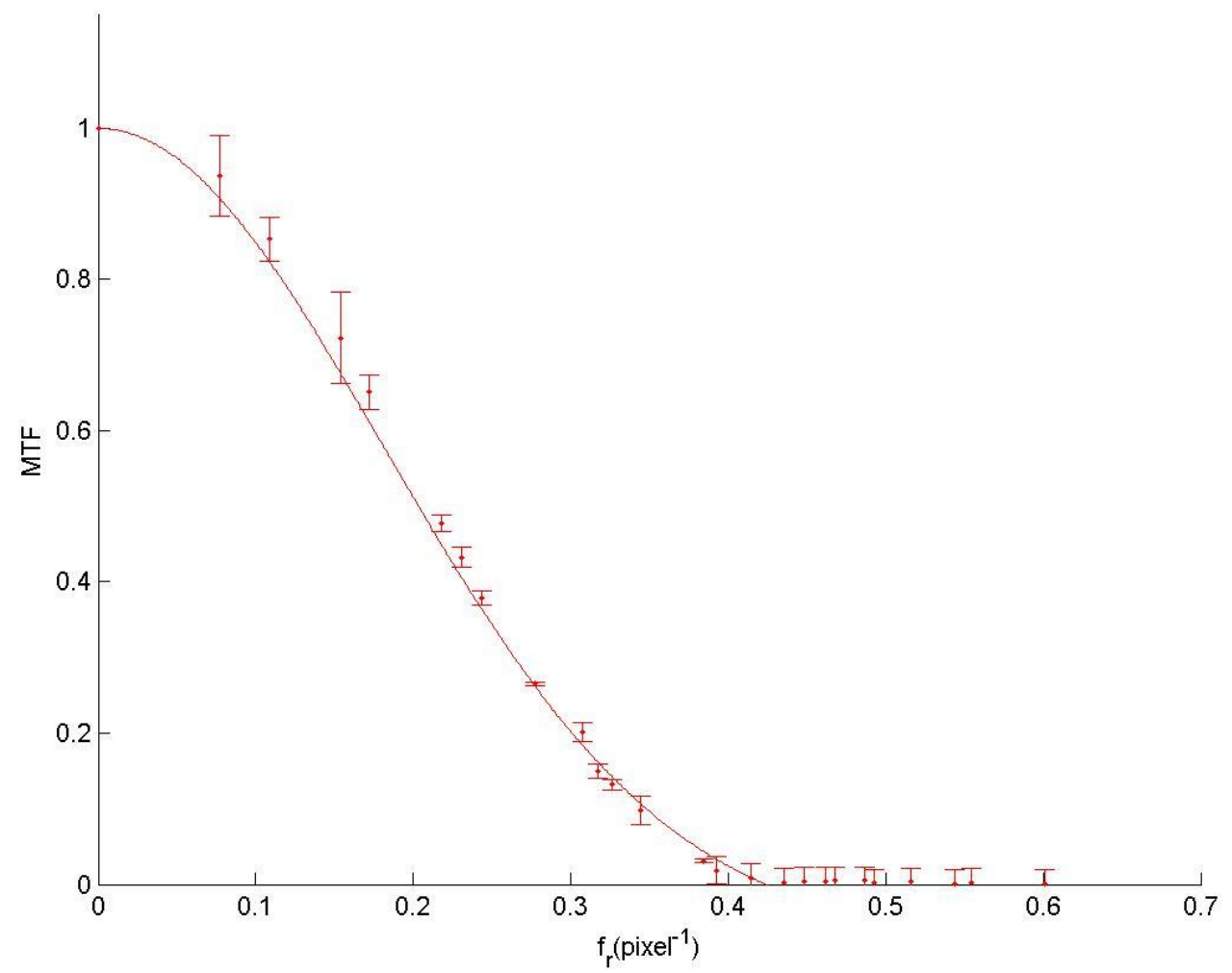

(b)

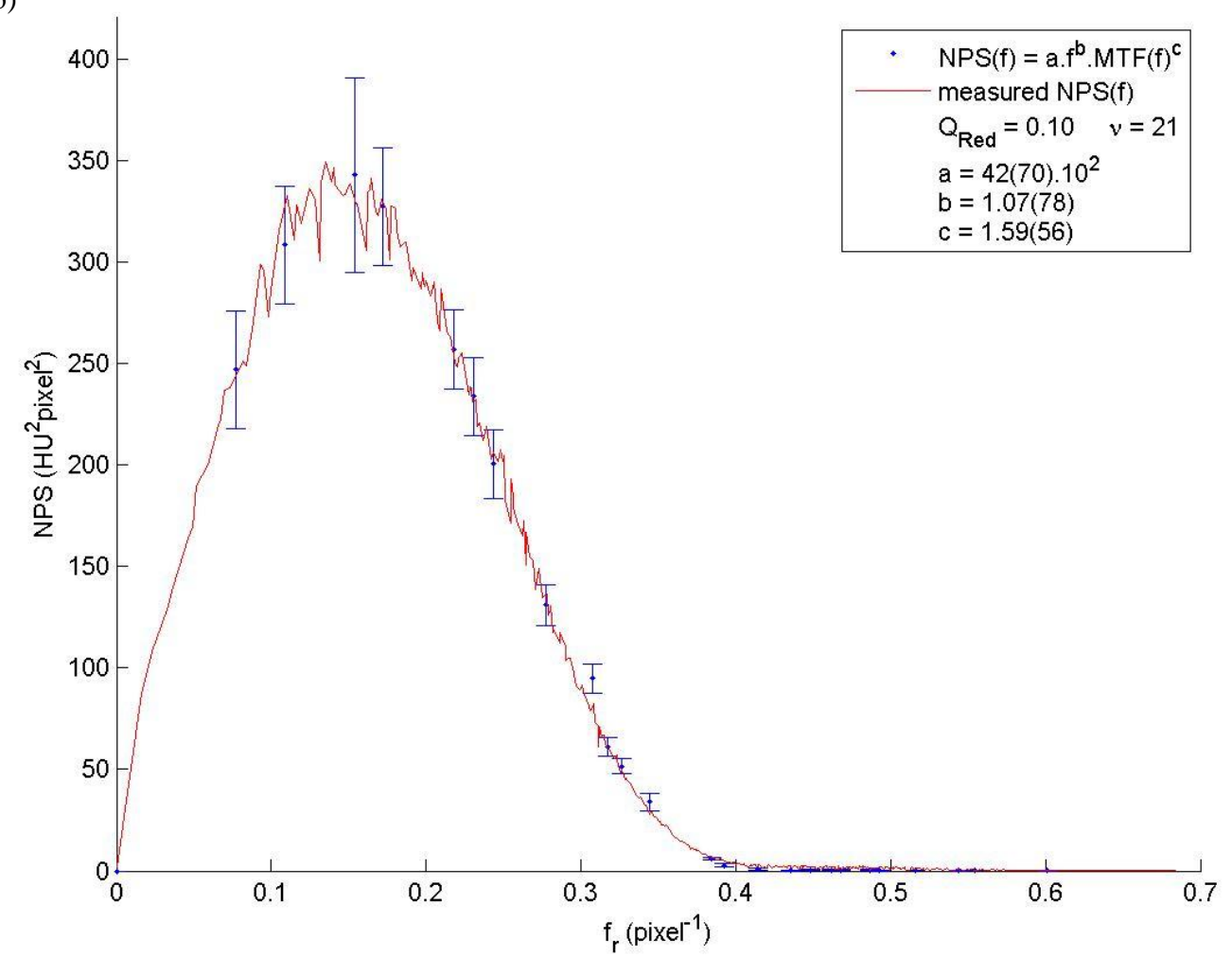

Figure 42: (a) MTF fitted by an illustrative Gaussian curve. (b) NPS measured at protocol 17 (GE Discovery750, axial mode, $1.25 \mathrm{~mm}$ slice thickness, $250 \mathrm{~mm}$ FOV) fitted as a function of the MTF. 
(a)

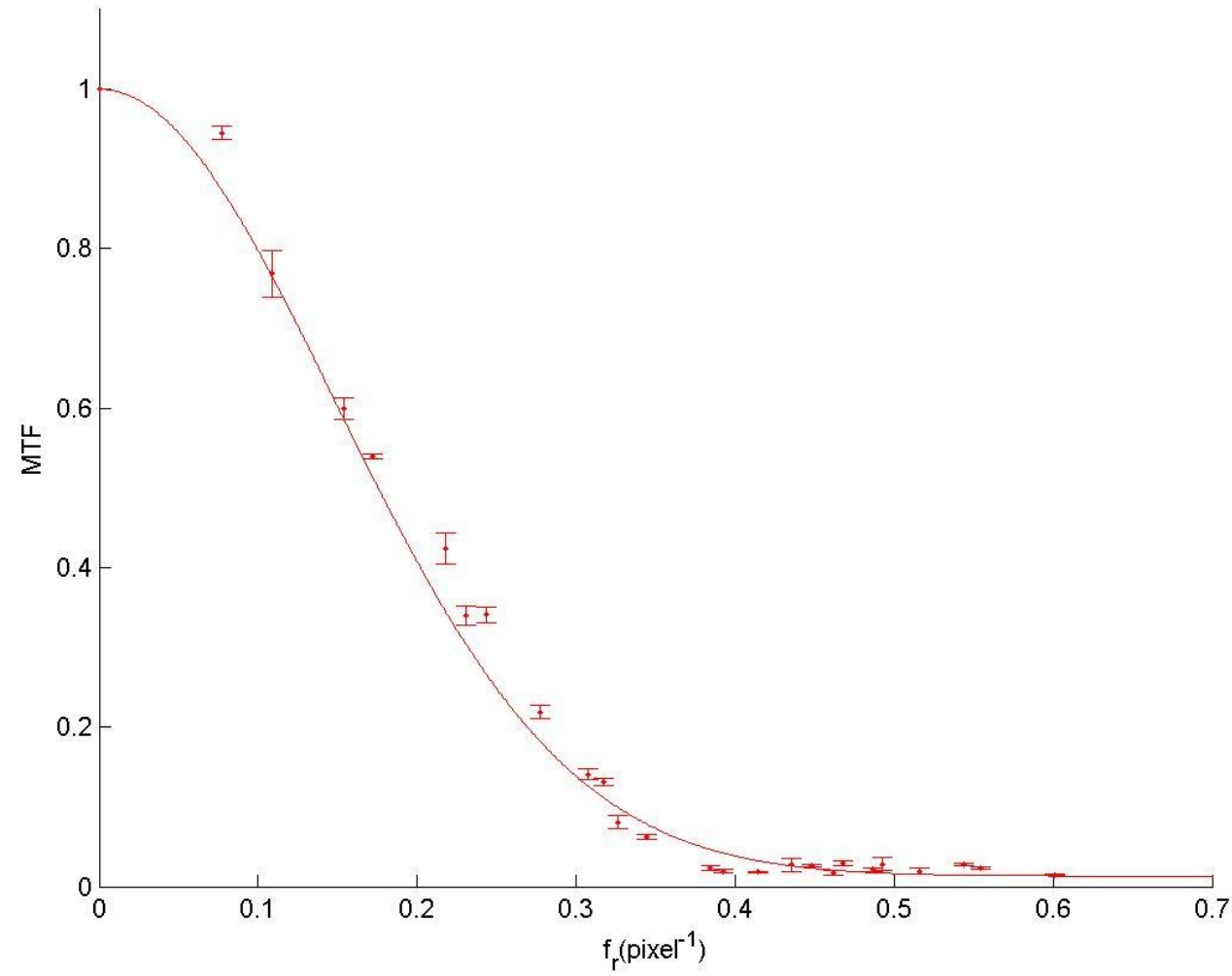

(b)

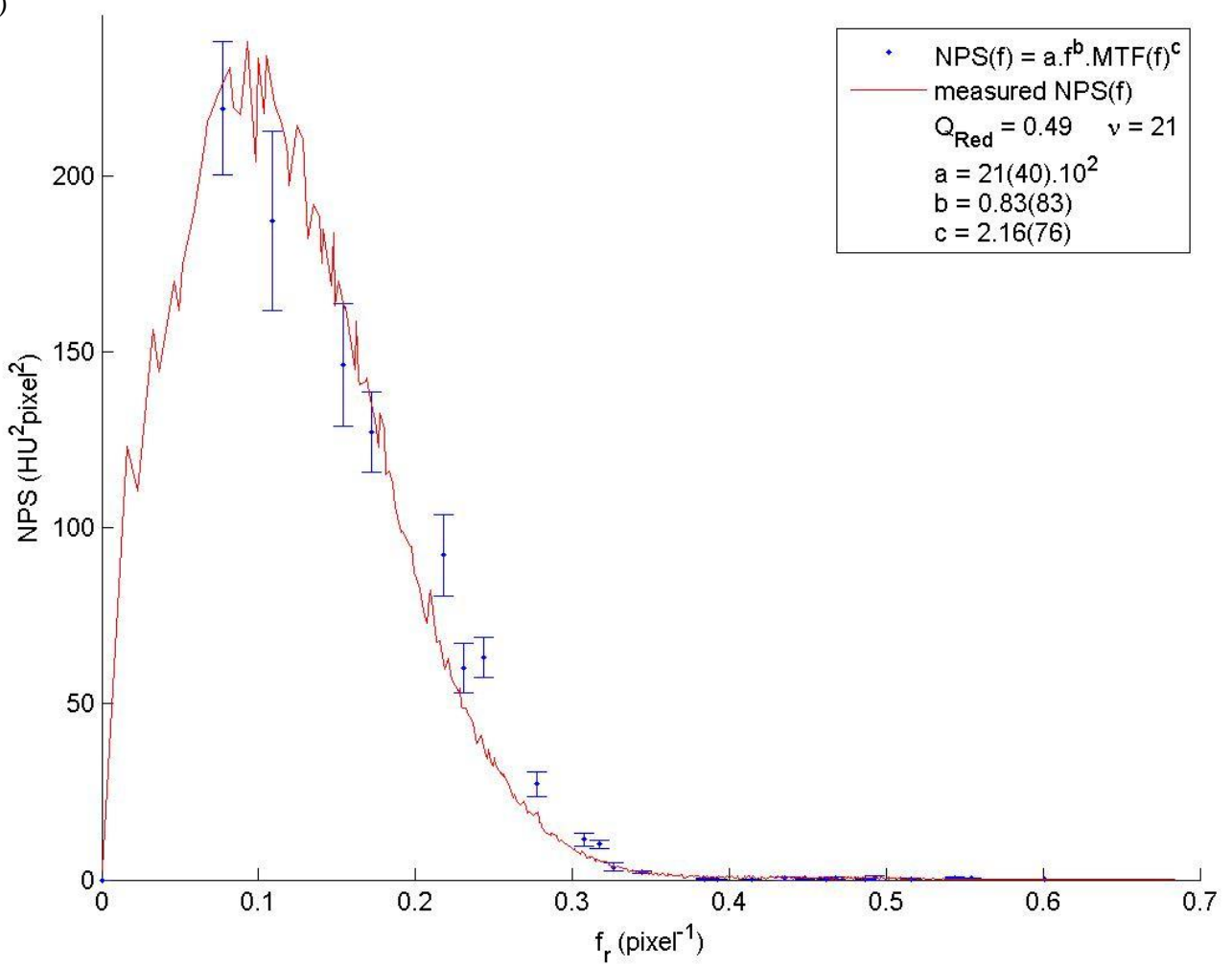

Figure 43: (a) MTF fitted by an illustrative Gaussian curve. (b) NPS measured at protocol 18 (GE Discovery750, helical mode, $2.5 \mathrm{~mm}$ slice thickness, $250 \mathrm{~mm}$ FOV) fitted as a function of the MTF. 
(a)

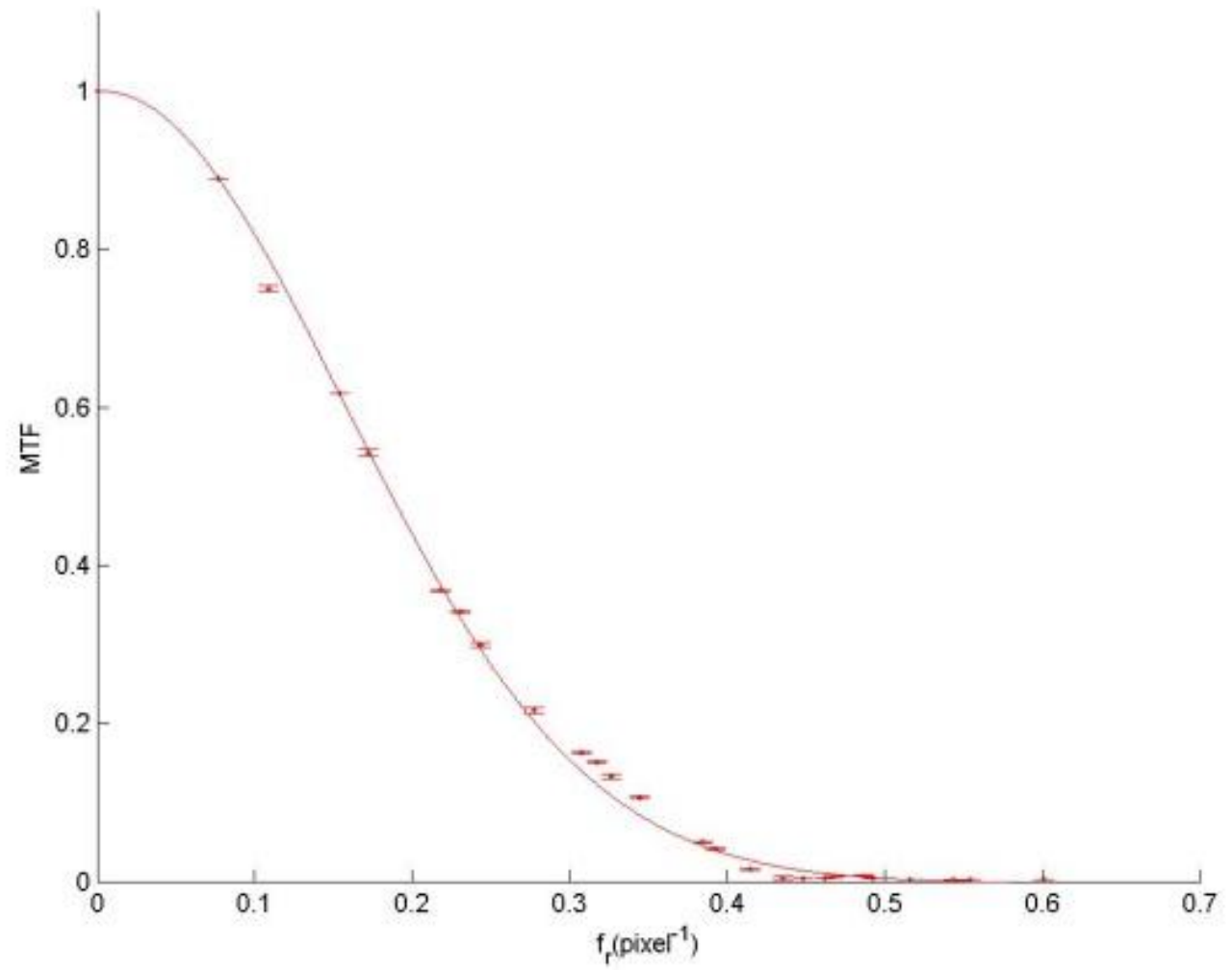

(b)

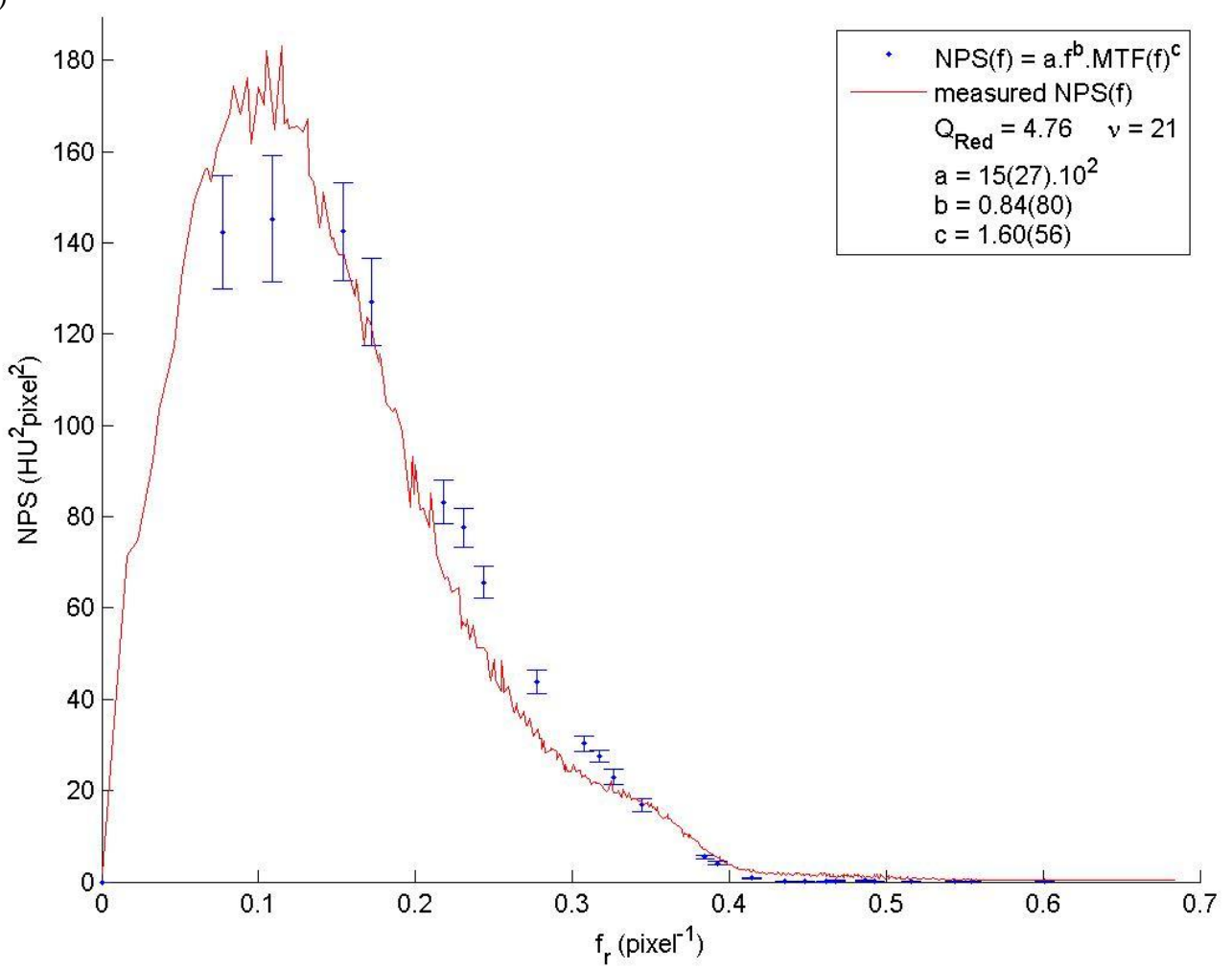

Figure 44: (a) MTF fitted by an illustrative Gaussian curve. (b) NPS measured at protocol 21 (Siemens Somatom Emotion16, 1.2mm slice thickness, 250mm FOV) fitted as a function of the MTF. 
(a)

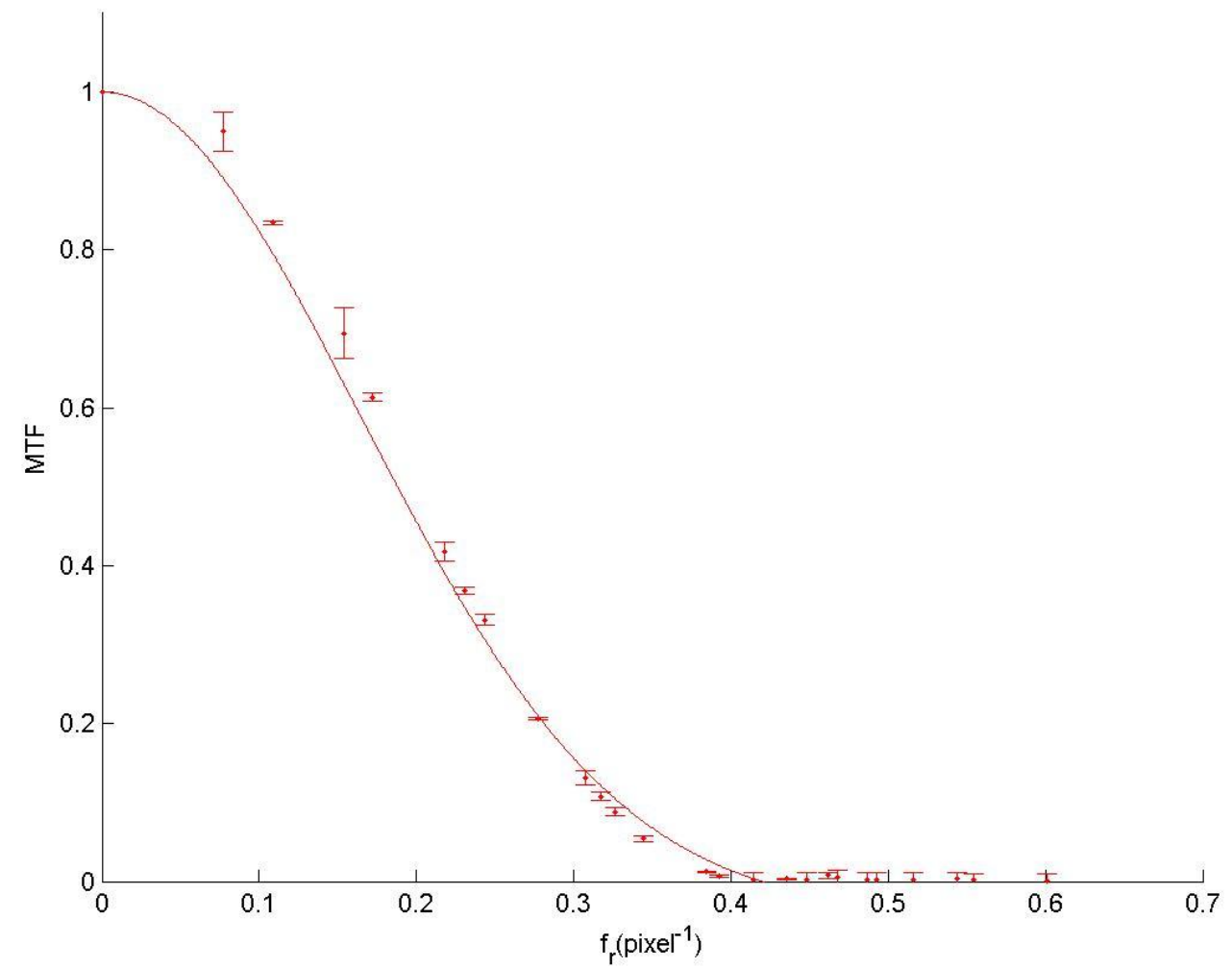

(b)

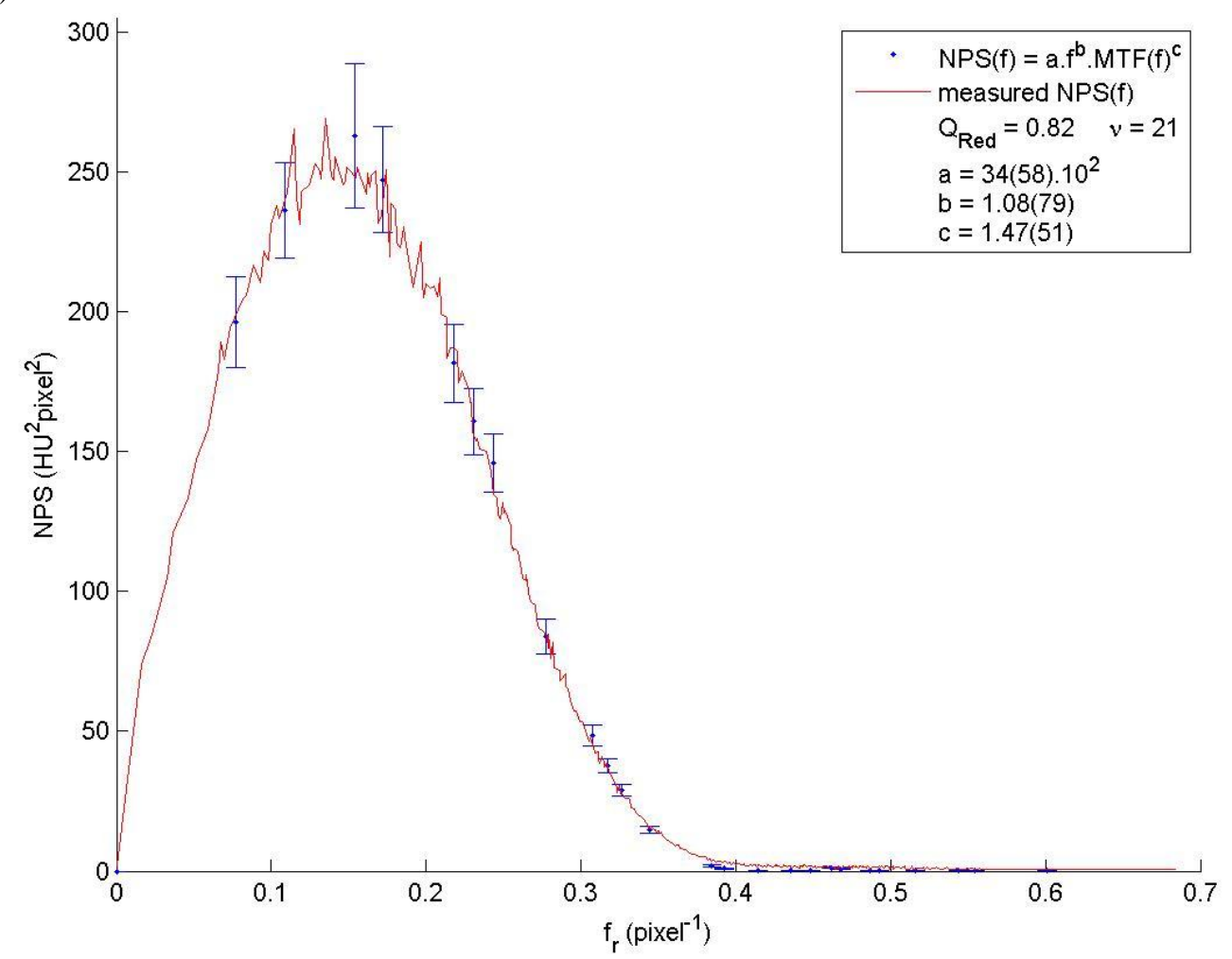

Figure 45: (a) MTF fitted by an illustrative Gaussian curve. (b) NPS measured at protocol 22 (GE LightspeedUltra, axial mode, $1.25 \mathrm{~mm}$ slice thickness, $240 \mathrm{~mm}$ FOV) fitted as a function of the MTF. 
(a)

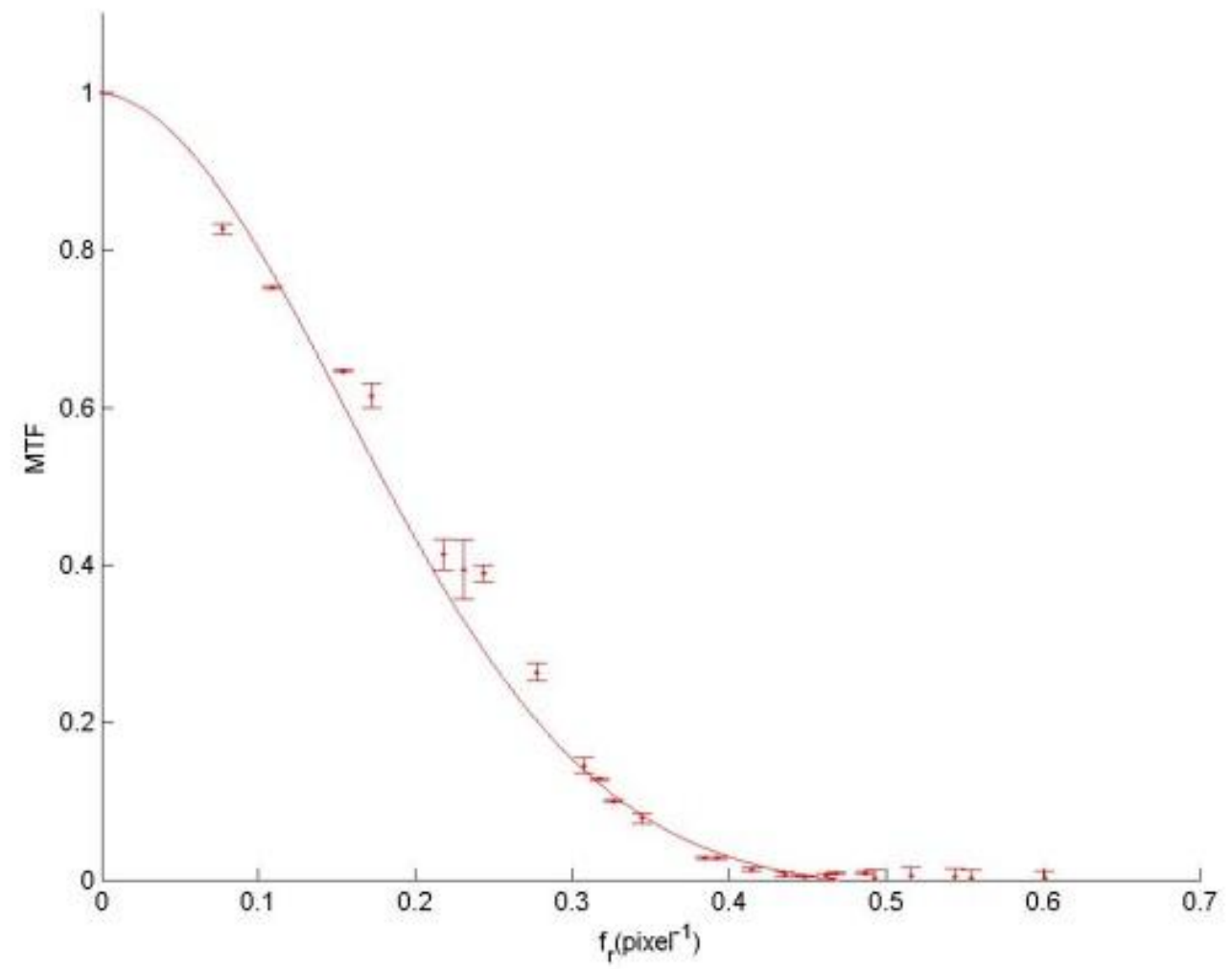

(b)

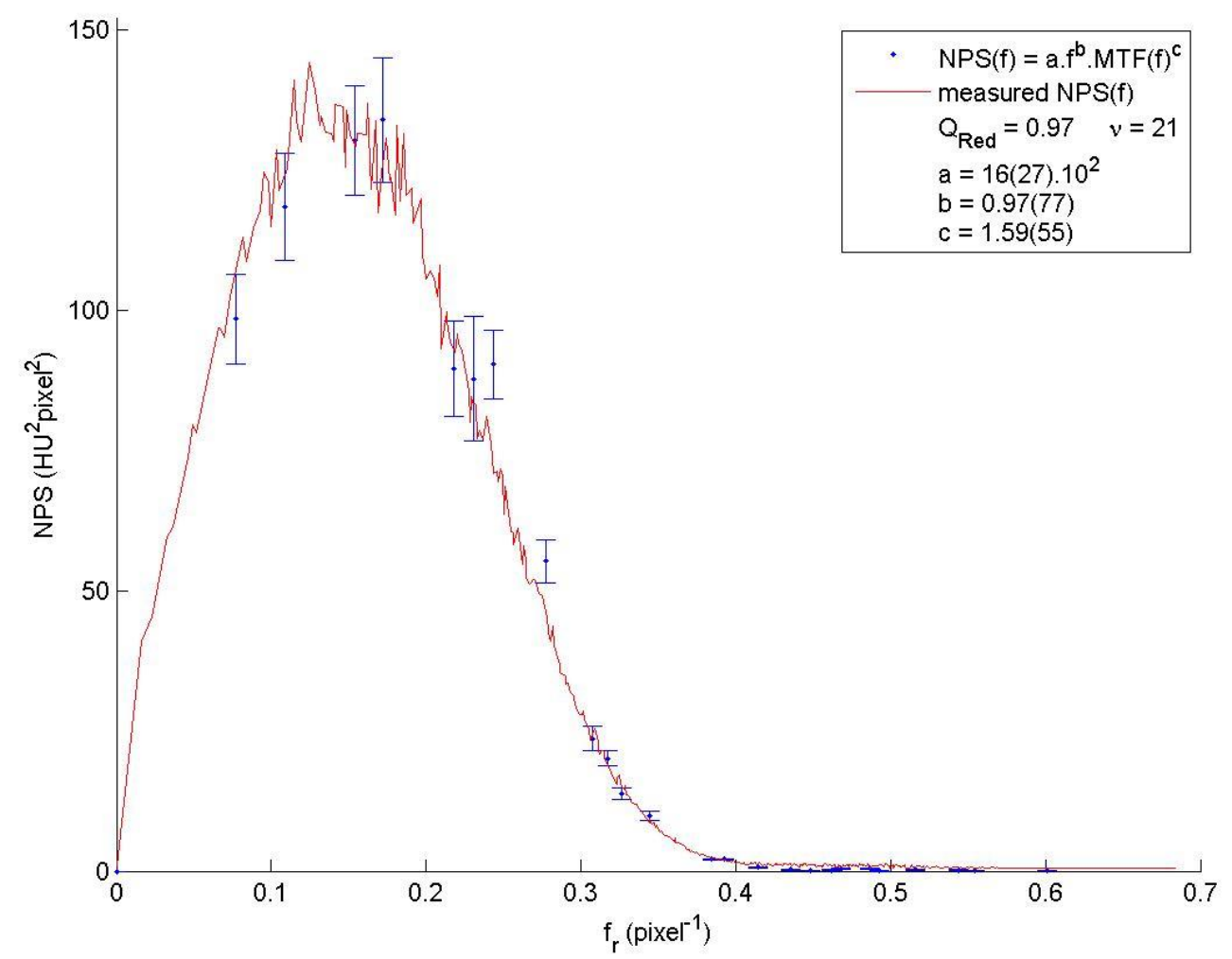

Figure 46: (a) MTF fitted by an illustrative Gaussian curve. (b) NPS measured at protocol 24 (GE LightspeedUltra, axial mode, $2.5 \mathrm{~mm}$ slice thickness, $250 \mathrm{~mm}$ FOV) fitted as a function of the MTF. 
(a)

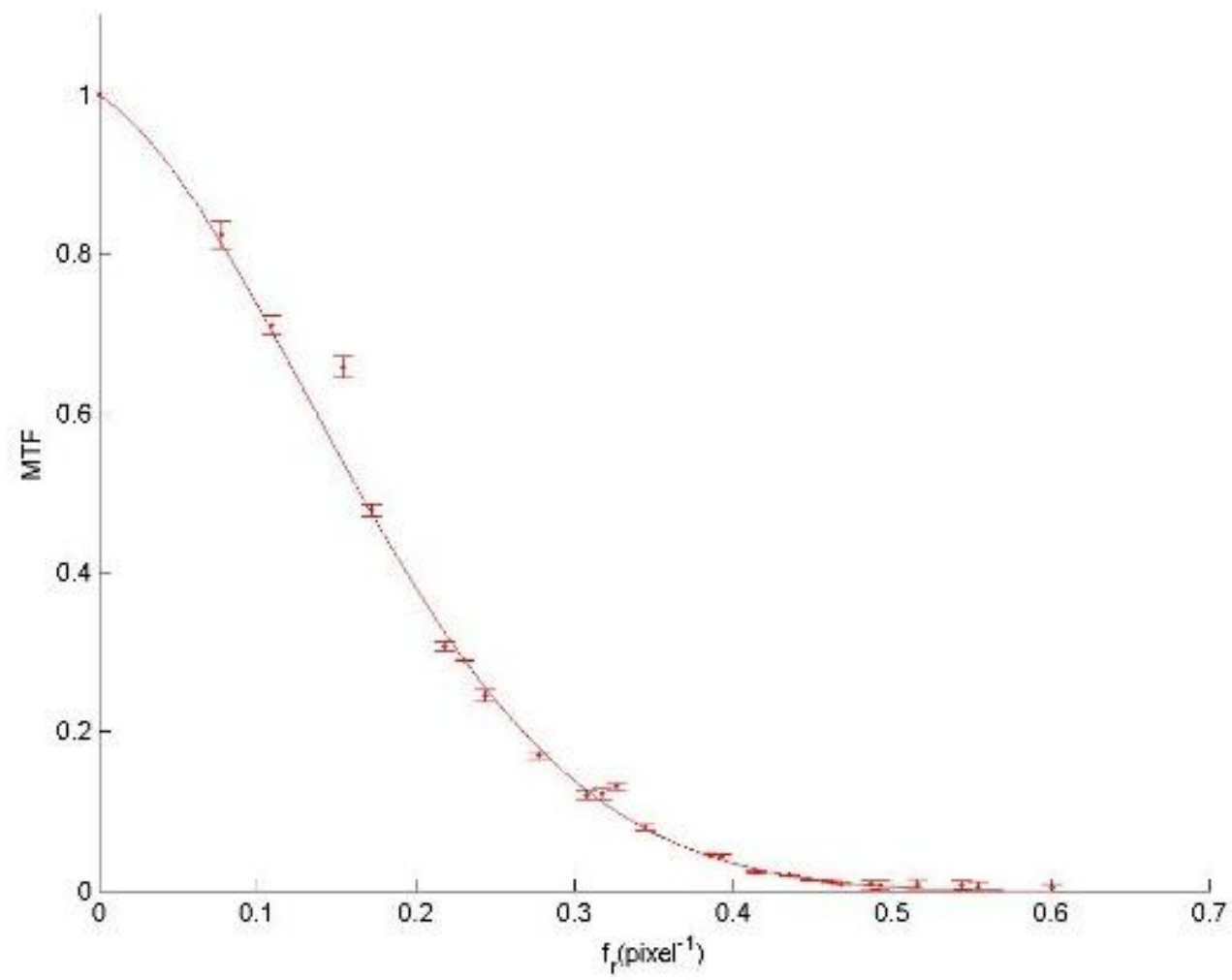

(b)

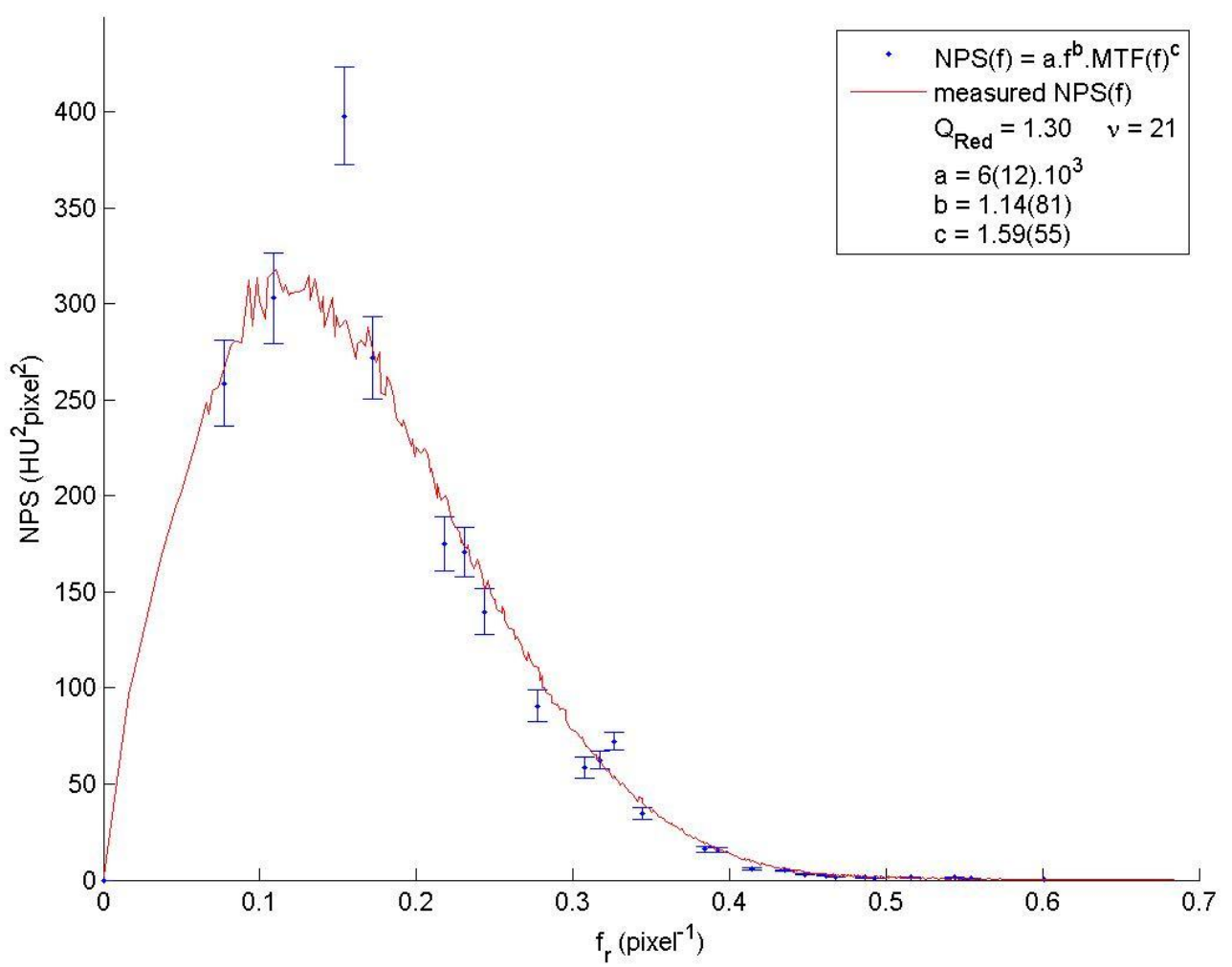

Figure 47: (a) MTF fitted by an illustrative Gaussian curve. (b) NPS measured at protocol 25 (Phillips Brilliance64, axial mode, $1.25 \mathrm{~mm}$ slice thickness, $250 \mathrm{~mm}$ FOV) fitted as a function of the MTF. 
(a)

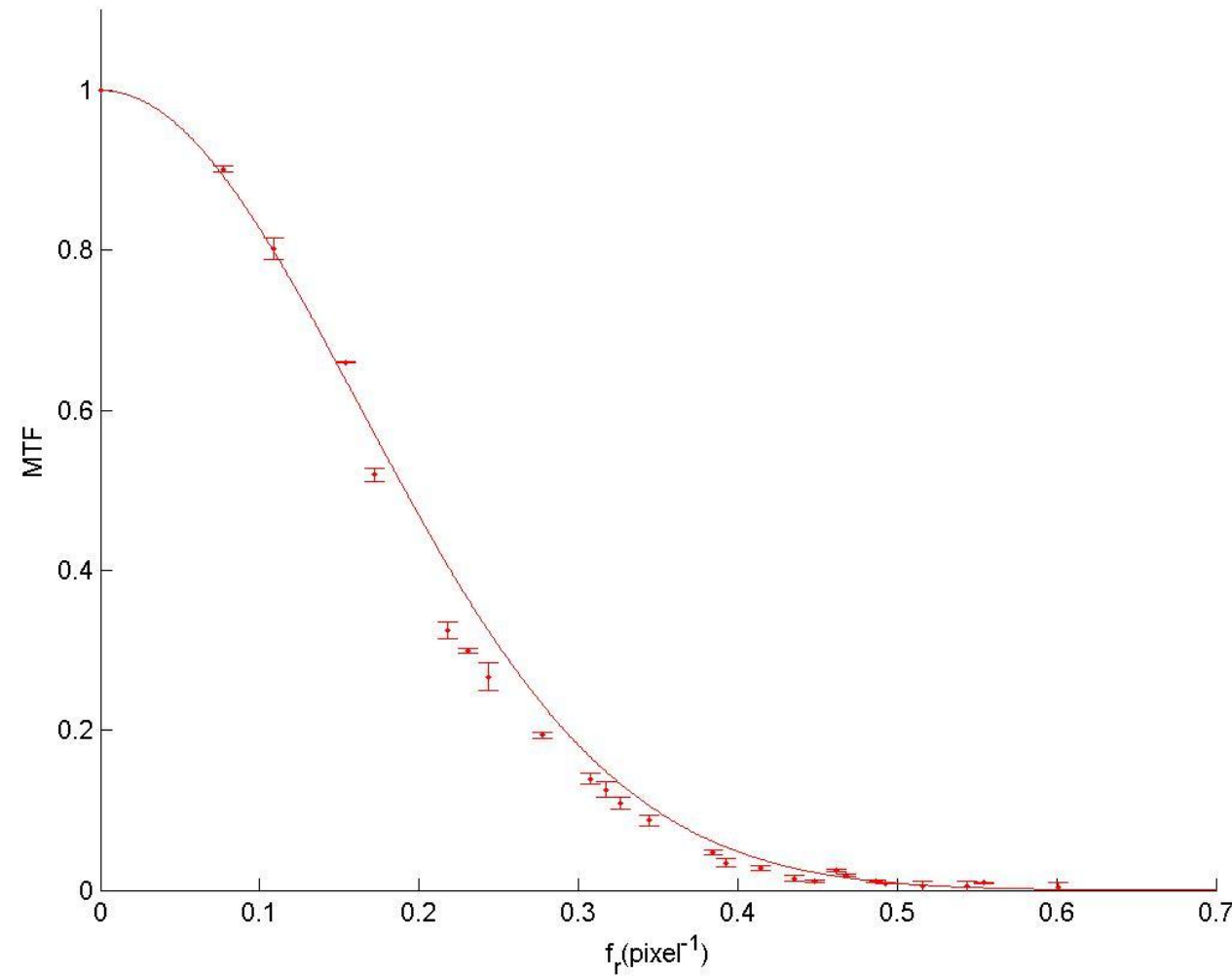

(b)

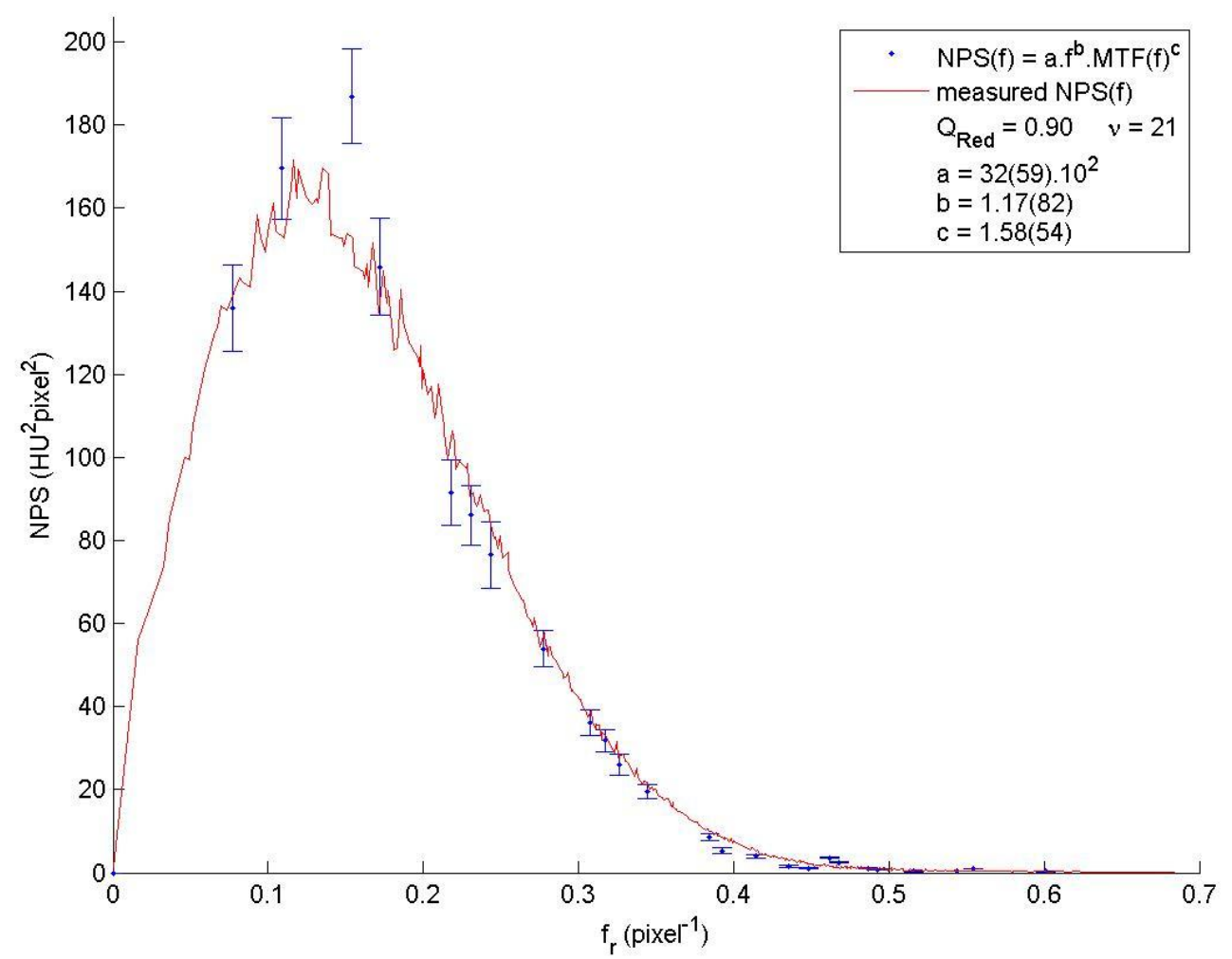

Figure 48: (a) MTF fitted by an illustrative Gaussian curve. (b) NPS measured at protocol 26 (Phillips Brilliance64, axial mode, $2.5 \mathrm{~mm}$ slice thickness, $250 \mathrm{~mm}$ FOV) fitted as a function of the MTF. 
These fitting results show the obtained MTF and NPS are consistent with the theoretical relation. The exponent $b$ agrees with the expected value from the theory (close to 1 ). The exponent $c$ seems slightly lower than expected (obtained around 1.5, the expected is 2 ), but the uncertainties on this parameter on all fits are large (from 0.5 to 0.6 ).

Protocol 18 is the only one that applies a different reconstruction algorithm, utilizing a combination of 50\% FBP and 50\% ASIR (iterative method). Even though, the employed fitting equation is adequate $\left(Q_{\text {red }}<1.56\right)$. The coefficients $b$ and $c$ obtained are slightly different from those of the other fits, but still within the expectation (given the large uncertainties).

In Figure 44, protocol 21 (the only protocol where $Q_{\text {red }}>Q_{\text {red,Crit }}$ ) shows an abnormal behavior in the NPS measurement (the subtle second peak between frequencies 0.3 and 0.4 pixel $^{-1}$ should not exist). The source of this anomaly is unknown. If this result were discarded as an acquisition mistake, then there are 1 in 12 protocols with $Q_{\text {red }}>$ 1.56; the probability of such result is $34.1 \%$ (binomial distribution, $p=0.05,12$ "attempts" and 1 "success").

\subsection{Software Performance}

As illustrated in Figure 8 at section 3.2, the software interface has two organization buttons, named "Sort" and "Identify" respectively. The algorithms of these functions are detailed in sections 3.2.1 to 3.2.3. The time the software takes to perform these two functions was estimated, using an Intel ${ }^{\circledR}$ i5 $3.2 \mathrm{GHz}$ processor with $8 \mathrm{~GB}$ of RAM memory and Windows ${ }^{\circledR} 7$ Ultimate Edition (64-bits). The other functions are executed much faster, therefore there was no need to evaluate their execution times. Table 3 presents the times measured with a number of test folders for the function "Sort". Table 4 presents this result for the function "Identify". Notice that, since the software speed is not dependent of the protocol, this was omitted and only the scanner model is presented at these tables. 
Table 3: Time spent per image by the function "Sort" measured for each test folder. Each test folder contained images of a different scanner, as presented in the first column. The last folder contained all the images from the previous folder. The last line of the table presents the resulting average (in ms/image) with the standard deviation.

\begin{tabular}{cccc}
\hline Scanner & Time (s) & Number of Images & $\begin{array}{c}\text { Time/image } \\
\text { (ms/image) }\end{array}$ \\
\hline BrillianceBigBore & 40 & 442 & 90.5 \\
\hline Discovery690 & 80 & 868 & 92.2 \\
\hline Aquillion & 42 & 624 & 67.3 \\
\hline Lightspeed & 38 & 418 & 90.9 \\
\hline All together & 230 & 2350 & 97.9 \\
\hline & & Average & $\mathbf{8 8}(\mathbf{1 2})$ \\
\hline
\end{tabular}

Table 4: Time spent per image by the function "Identify" measured for each test folder. Each test folder contained images of a different scanner, as presented in the first column. The last line of the table presents the resulting average (in ms/image) with the standard deviation.

\begin{tabular}{cccc}
\hline Scanner & Time (s) & Number of Images & $\begin{array}{c}\text { Time/image } \\
\text { (ms/image) }\end{array}$ \\
\hline Bigbore & 41 & 80 & 513 \\
\hline Bigbore & 67 & 140 & 479 \\
\hline Bigbore & 31 & 79 & 392 \\
\hline Bigbore & 49 & 140 & 350 \\
\hline Discovery690 & 72 & 160 & 450 \\
\hline Discovery690 & 25 & 69 & 362 \\
\hline Aquillion & 12 & 36 & 333 \\
\hline Aquillion & 9 & 32 & 281 \\
\hline Aquillion & 53 & 166 & 319 \\
\hline Lightspeed & 45 & 96 & 469 \\
\hline Lightspeed & 23 & 64 & 359 \\
\hline Lightspeed & 24 & 69 & $\mathbf{3 8 8}(\mathbf{7 2})$ \\
\hline
\end{tabular}

Table 3 and Table 4 show the speed of the Sort and Identify functions are fairly consistent, using approximately 90 and $400 \mathrm{~ms}$ per image of the containing folder regardless of the quantity of images. 


\section{CONCLUSIONS}

The objective of this work was writing a software in MatLab ${ }^{\circledR}$ to analyze images of the Catphan $5000^{\circledR} \mathrm{CT}$ phantom, improving the quality control process. The software should have a graphical interface, meant to be intuitive even for a user unfamiliar with $\mathrm{MatLab}^{\circledR}$, and should work with minimal user interference, reducing the subjectivity of some parts of the quality control procedures as well as the chance of human errors. Additionally, the software should be validated, assuring potential users that its outputs are in accordance with the results obtained by qualified professionals using traditional methods. These goals were achieved, and the resulting software is ready to be applied to the quality control process.

The software uses the input images from the phantom to calculate slice thickness, slice increment and pixel size, and evaluate the calibration of the CT number. These parameters are chosen by the user at the moment of image acquisition, and are measured in CT quality control programs, as they indicate if the scanner is functioning properly.

It also estimates the Modulation Transfer Function $(M T F)$, the Noise Power Spectrum (NPS) and the noise magnitude. From the MTF, the software also calculates the cutoff frequency and the frequency at $10 \%$ of the MTF of the system. These parameters are important in the assessment of the quality of the images produced by the scanner under evaluation, and therefore they indicate how much diagnostic information these images provide.

It was also performed the validation of the software; image sets from 10 different scanners, taken at 27 different acquisition conditions, were used to test the software. The results from the software output were compared with the results presented in the quality control reports of these same scanners.

For pixel size, slice thickness, slice increment and the fitting coefficients of the CT number calibration, the t-test was applied between software and quality control report results, with p-value of 0.01 . For the noise magnitude, the F-test was applied, with a p-value of 0.05 (since noise is estimated by a standard deviation, the F-test is more appropriate than the t-test). These tests showed that the results obtained by the software are, at least, as good as the results obtained by the normal quality control procedures.

The cutoff frequency and frequency at $10 \%$ of the MTF were compared with the cutoff frequency presented in the quality control report. But these three values are calculated by very different methods (the first two are obtained by the MTF and are already 
different by definition, and the third is obtained visually from a set of bar patterns contained in the phantom). Therefore, a statistical test was deemed inappropriate to compare them. So, these frequencies were compared directly in a single plot. It was found that the visual estimation performed by the quality control routine is consistently higher than the frequency at $10 \%$ calculated by the software, with few exceptions. Also, the cutoff frequency is always the highest value, which is consistent with the expected from the theory. However, the uncertainties of the cutoff frequency were shown to be very hard to estimate, because this value is in a region of the $M T F$ where it fluctuates so close to zero. These uncertainty values varied within three orders of magnitude between different measurements, too large to draw conclusions about how "right" the estimates of the cutoff frequency are. Even so, comparing the cutoff values with the MTF graphs by a visual criterion, they are not unreasonable.

The MTF and NPS functions are not measured by normal quality control procedures, so there is no comparison to be done. However, they can be analyzed against each other, using the theoretical relation between MTF and NPS of CT systems. So, the NPS was fitted as a function of the $M T F$; the obtained parameters were compared with the theory and the goodness of the fit evaluated by the chi-square test. Through this, the MTF and NPS obtained were shown to be consistent with each other and agree with the theoretical predictions.

There is a range of possible future works. The software could be adapted or expanded to assess image quality using CT images of other similar phantoms, such as the Catphan $600^{\circledR}$ and Catphan $700^{\circledR}$. It could also be expanded to calculate more variables that are interesting to QC programs, such as the low contrast resolution or incorporate the z-axis into the MTF and NPS. Using similar methods, a new software could be written to evaluate image quality of other radiological modalities (e.g., conventional radiography, mammography, etc). Lastly, the software might be tested in a broader range of CT scanners in order to better define its limitations, and possibly improve on them. 


\section{APPENDIX A: DIGITAL SYSTEMS}

Of all imaging systems, digital systems are particularly important. When working on digital data, the choice of filters is completely up to the user, any transformation is possible, including non-linear operations. However, digital systems have the quirk of always being discrete; so, it is necessary a method to faithfully represent continuous

functions with discrete data. Formally: given a continuous function $f(\boldsymbol{r})$, obtain a discrete function $f_{s}\left(\boldsymbol{r}_{j}\right)$, from which it is possible to perfectly reconstruct $f(\boldsymbol{r})$ whenever desired, and define the method for doing $\mathrm{it}^{27}$.

\section{Sampling}

The Comb function is an infinite sum of $n$-dimensional delta functions spaced of 1 unit:

$$
c(\boldsymbol{r})=\sum_{\boldsymbol{j}=-\infty}^{\boldsymbol{j}=\infty} \delta^{n}(\boldsymbol{r}-\boldsymbol{j})
$$

This function has the property:

$$
\begin{gathered}
\mathcal{F}_{\boldsymbol{k}}[c(\boldsymbol{r})]=\sum_{\boldsymbol{j}=-\infty}^{j=\infty} \delta^{n}(\boldsymbol{k}-\boldsymbol{j}) \\
\mathcal{F}_{\boldsymbol{k}}[c(\boldsymbol{r})]=c(\boldsymbol{k})
\end{gathered}
$$

The Comb function can be used to select points (spaced of $\Delta$ ) in a signal $s(\boldsymbol{r})$ :

$$
s_{S}(\boldsymbol{r})=\Delta^{-n} s(\boldsymbol{r}) c(\boldsymbol{r} / \Delta)
$$

In other words, it is possible to use the Comb function to sample a continuous signal and make it discrete. It is still unknown the spacing $\Delta$ that is capable of recreating the original, and the method to do it. 


\section{Nyquist Frequency}

Taking the Fourier transform of the sampled signal:

$$
\begin{gathered}
S_{S}(\boldsymbol{k})=S(\boldsymbol{k}) * c(\Delta \boldsymbol{k}) \\
S_{S}(\boldsymbol{k})=\Delta^{-n} \sum_{\boldsymbol{j}} S\left(\boldsymbol{k}-\boldsymbol{j} \Delta^{-n}\right)
\end{gathered}
$$

Being $\boldsymbol{j}$ a vector where each component goes from $-\infty$ a $\infty$. This result shows that the sampled signal in the frequency space $S_{s}(\boldsymbol{k})$ is an infinite superposition of copies of the original signal $S(\boldsymbol{k})$, displaced of $\Delta^{-1}$ between themselves in each dimension. In the case these copies do not overlap each other (i.e., the signal is band limited):

$$
S(\boldsymbol{k})=0 \text { if }\left|k_{i}\right|>k_{\max } \quad i=1,2 \ldots, n
$$

So, in order to the copies of $S(\boldsymbol{k})$ in $S_{s}(\boldsymbol{k})$ to not overlap, the maximum spatial frequency of the signal $k_{\max }$ and the spacing of the sample must obey the relation:

$$
\Delta^{-1} \geq 2 k_{\max }
$$

This way, the sampling rate $\Delta^{-1}$ must be at least twice the maximum frequency of the sampled signal. This frequency is called the Nyquist frequency, or the cutoff frequency. If this relation is true, the original signal can be obtained from the sampled signal without loss of information.

Rigorously, no signal that is spatially limited (like an image, finite) can also be band limited (have a well determined maximum frequency), but generally it is a good approximation.

\section{Whittaker-Shannon Theorem}

In the case of (approximately) band limited signals, it is possible to pick a single copy of $S(\boldsymbol{k})$ in $S_{s}(\boldsymbol{k})$ applying a $n$-dimensional rectangular function to $S_{s}(\boldsymbol{k})$ : 


$$
S_{S}(\boldsymbol{k}) \cdot \operatorname{rect}(\boldsymbol{k} \Delta)=\Delta^{-n} S(\boldsymbol{k})
$$

Where $\operatorname{rect}(x)$ and its Fourier transform are defined:

$$
\begin{gathered}
\operatorname{rect}(x)=\left\{\begin{array}{l}
1 \text { se }|x| \leq 1 / 2 \\
0 \text { se }|x|>1 / 2
\end{array}\right. \\
\mathcal{F}_{v}[\operatorname{rect}(x)]=\operatorname{sinc}(x)=\frac{\sin (\pi x)}{\pi x}
\end{gathered}
$$

Going back to the position space:

$$
s_{s}(\boldsymbol{r}) * \operatorname{sinc}(\boldsymbol{r} / \Delta)=s(\boldsymbol{r})
$$

In a more explicit form:

$$
\sum_{\boldsymbol{j}} s\left(\boldsymbol{r}_{\boldsymbol{j}}\right) \cdot \operatorname{sinc}\left(\frac{\boldsymbol{r}-\boldsymbol{r}_{\boldsymbol{j}}}{\Delta}\right)=s(\boldsymbol{r})
$$

This formula is the Whitaker-Shannon theorem; it is valid for band limited signals (or approximately so) and sampled according to the Nyquist frequency.

It must be remembered that this is an interpolation rule, and so it is not unique; to deduce it, it was chosen a region defined by the rectangular function to pick a single copy of the signal. Any other choice (a circular region, for example) that also selects a single copy is equally valid, and results in a different interpolation rule.

\section{Aliasing}

When the sampling rate is lower than twice the Nyquist frequency, the WhittakerShannon rule does not work; high spatial frequencies are interpolated as if they were low frequencies, information is lost, and false information may be introduced. 


\section{APPENDIX B: PROBABILITY THEORY}

Notation: stochastic variables are written in normal style instead of italic, to distinguish from deterministic variables.

\section{Probability}

There are many equivalent definitions of probability; here, the interest is in the frequentist definition. Given an experiment $\mathrm{A}$, done $N$ times, the probability of obtaining the particular result $\mathrm{A}_{\mathrm{i}}$ is:

$$
\operatorname{Pr}\left(\mathrm{A}_{\mathrm{i}}\right)=\lim _{N \rightarrow \infty} \frac{\mathrm{n}\left(\mathrm{A}_{\mathrm{i}}\right)}{N}
$$

Where $n\left(A_{i}\right)$ is the number of times the test resulted in $A_{i}$.

From this definition follows that any probability is a positive number, and the probability of obtaining any result is 1 (normalization rule):

$$
\sum_{i} \operatorname{Pr}\left(\mathrm{A}_{\mathrm{i}}\right)=1
$$

Give 2 experiments $A$ and $B$, the probability of the results $A_{i}$ and $B_{j}$ occurring is:

$$
\operatorname{Pr}\left(A_{i} \cap B_{j}\right)=\operatorname{Pr}\left(A_{i} \mid B_{j}\right) \operatorname{Pr}\left(B_{j}\right)
$$

Where $\operatorname{Pr}\left(A_{i} \mid B_{j}\right)$ is the conditional probability of $A_{i}$ to $B_{j}$, i.e., the probability of $A_{i}$ occurring given that $\mathrm{B}_{\mathrm{j}}$ occurred. If the experiments $\mathrm{A}$ and $\mathrm{B}$ are statistically independents, the result of $\mathrm{B}$ does not have any influence over the possible results of $\mathrm{A}$ (and vice-versa), so:

$$
\operatorname{Pr}\left(A_{i} \mid B_{j}\right)=\operatorname{Pr}\left(A_{i}\right) \rightarrow \operatorname{Pr}\left(A_{i} \cap B_{j}\right)=\operatorname{Pr}\left(A_{i}\right) \operatorname{Pr}\left(B_{j}\right)
$$

In an experiment $A$, the probability of any 2 results $A_{i}$ or $A_{j}$ occurring is: 


$$
\operatorname{Pr}\left(A_{i} \cup A_{j}\right)=\operatorname{Pr}\left(A_{i}\right)+\operatorname{Pr}\left(A_{j}\right)-\operatorname{Pr}\left(A_{i} \cap A_{j}\right)
$$

In the case these results are mutually exclusive (the occurrence of one makes the other impossible):

$$
\operatorname{Pr}\left(A_{i} \cap A_{j}\right)=0 \rightarrow \operatorname{Pr}\left(A_{i} \cup A_{j}\right)=\operatorname{Pr}\left(A_{i}\right)+\operatorname{Pr}\left(A_{j}\right)
$$

Notice that $A_{i}$ is not necessarily a number, it is just a possible result of the procedure $A$. If A is "flip a coin", for example, the results $A_{1}$ and $A_{2}$ are "heads" and "tails" respectively, no numbers involved. So, $\operatorname{Pr}\left(\mathrm{A}_{\mathrm{i}}\right)$ is not to be read as "function of $\mathrm{A}_{\mathrm{i}}$ ", at least not in a mathematically rigorous way. If there is a numeric representation of each $\mathrm{A}_{\mathrm{i}}$, it is said $\mathrm{A}$ is a stochastic variable, or random variable; then, it is possible to project these concepts to continuous variables.

\section{Probability Density and Moments}

If a stochastic variable $\mathrm{x}$ is continuous, there is an infinite number of possible values it can take, so the probability of any particular value $\mathrm{x}_{\mathrm{i}}$ is zero. However, it is possible to define the probability of obtaining $x$ inside a small finite interval $\left[\mathrm{x}_{\mathrm{i}}, \mathrm{x}_{\mathrm{i}}+\mathrm{dx}\right]$ :

$$
\operatorname{Pr}\left(\left[\mathrm{x}_{\mathrm{i}}, \mathrm{x}_{\mathrm{i}}+\mathrm{dx}\right]\right)=\rho\left(\mathrm{x}_{\mathrm{i}}\right) \mathrm{dx}
$$

The function $\rho(\mathrm{x})$ is called probability distribution, or probability density. Unlike $\operatorname{Pr}\left(\mathrm{A}_{\mathrm{i}}\right)$, $\rho(\mathrm{x})$ is actually a function of $\mathrm{x}$. For a generic interval $\left[\mathrm{x}_{\mathrm{a}}, \mathrm{x}_{\mathrm{b}}\right]$ :

$$
\operatorname{Pr}\left(\left[\mathrm{x}_{\mathrm{a}}, \mathrm{x}_{\mathrm{b}}\right]\right)=\int_{\mathrm{x}_{\mathrm{a}}}^{\mathrm{x}_{\mathrm{b}}} \rho(\mathrm{x}) \mathrm{dx}
$$

Being probabilities always positive, $\rho(\mathrm{x})$ must also be. For the interval $(-\infty, \infty)$ (all possible values of $\mathrm{x}$ ), the normalization rule becomes:

$$
\operatorname{Pr}([-\infty, \infty])=\int_{-\infty}^{\infty} \rho(\mathrm{x}) \mathrm{dx}=1
$$


Similarly, all other results shown above for discrete variables apply to continuous variables. Besides, it is possible to use the equations for continuous variables to work discretely, if the function $\rho(\mathrm{x})$ is a succession of Dirac deltas over the possible values of $\mathrm{x}$. For this reason, the continuous form will be preferred from now on, unless the discrete form is really necessary.

The mean of $\mathrm{x}$, denoted $\langle\mathrm{x}\rangle$ ou $\overline{\mathrm{x}}$, is defined as:

$$
\langle\mathrm{x}\rangle=\int_{-\infty}^{\infty} \mathrm{x} \rho(\mathrm{x}) \mathrm{dx}
$$

Also the variance of $\mathrm{x}, \sigma_{\mathrm{x}}^{2}$ :

$$
\sigma_{\mathrm{x}}^{2}=\left\langle(\mathrm{x}-\langle\mathrm{x}\rangle)^{2}\right\rangle=\left\langle\mathrm{x}^{2}\right\rangle-\langle\mathrm{x}\rangle^{2}=\int_{-\infty}^{\infty} \mathrm{x}^{2} \rho(\mathrm{x}) \mathrm{d} \mathrm{x}-\left(\int_{-\infty}^{\infty} \mathrm{x} \rho(\mathrm{x}) \mathrm{dx}\right)^{2}
$$

The same way one can define the $n$-th moment of the distribution:

$$
\left\langle\mathrm{x}^{n}\right\rangle=\int_{-\infty}^{\infty} \mathrm{x}^{n} \rho(\mathrm{x}) \mathrm{dx}
$$

And the $n$-th central moment: $\left\langle(\mathrm{x}-\langle\mathrm{x}\rangle)^{n}\right\rangle$.

For distributions of 2 or more variables $\rho(\mathrm{x}, \mathrm{y})$, the moments and central moments are $\left\langle\mathrm{x}^{n} \mathrm{y}^{m}\right\rangle$ e $\left\langle(\mathrm{x}-\langle\mathrm{x}\rangle)^{n}(\mathrm{y}-\langle\mathrm{y}\rangle)^{m}\right\rangle$ respectively.

The moments are useful in many ways. Firstly, some moments (the first ones in particular) have an interpretable meaning; the first moment is the mean, the expected value of the variable $\mathrm{x}$; the second central moment is the variance, a measure of the dispersion of the values of $\mathrm{x}$ around its mean; other moments also have their own meaning. 
Besides, the moments are generally easier to estimate than the distribution itself. Often just a few data are enough for a good estimative of mean and variance (and often it is all that is needed), while estimating the distribution would require thousands of data points. Lastly, having all moments gives the distribution. The characteristic function $\wp(\mathrm{k})$ is defined as:

$$
\wp(\mathrm{k})=\mathcal{F}_{\mathrm{k}}[\rho(\mathrm{x})]=\left\langle e^{-2 \pi i \mathrm{kx}}\right\rangle
$$

The characteristic function is also called the moment generating function, for it relates to the $n$-th moment by the relation:

$$
\left.\left(\frac{\mathrm{d}^{n}}{\mathrm{dk}^{n}} \wp(\mathrm{k})\right)\right|_{\mathrm{k}=0}=(-2 \pi i)^{n}\left\langle\mathrm{x}^{n}\right\rangle
$$

The left side is the $n$-th Taylor expansion coefficient of the characteristic function, so $\wp(\mathrm{k})$ is completely described by the moments. And having $\wp(\mathrm{k}), \rho(\mathrm{x})$ is obtained by the inverse Fourier transform.

Another important result obtained using the characteristic function is the distribution of a sum of independent random variables. Given $\mathrm{z}=\mathrm{x}+\mathrm{y}$, where $\mathrm{x}$ and $\mathrm{y}$ are independent and have distributions $\rho_{\mathrm{x}}(\mathrm{x})$ and $\rho_{\mathrm{y}}(\mathrm{y})$ respectively:

$$
\begin{gathered}
\wp_{\mathrm{z}}(\mathrm{k})=\left\langle e^{-2 \pi i \mathrm{k}(\mathrm{x}+\mathrm{y})}\right\rangle=\left\langle e^{-2 \pi i \mathrm{kx}}\right\rangle\left\langle e^{-2 \pi i \mathrm{ky}}\right\rangle=\wp_{\mathrm{x}}(\mathrm{k}) \cdot \wp_{\mathrm{y}}(\mathrm{k}) \\
\rho_{\mathrm{z}}(\mathrm{z})=\rho_{\mathrm{x}}(\mathrm{z}) * \rho_{\mathrm{y}}(\mathrm{z})
\end{gathered}
$$

In words, the probability density of a sum of random variables is the convolution of the probability densities of each variable.

\section{Stochastic Processes}

If the behavior of a stochastic variable $\mathrm{x}$ depends on a control parameter $u$ (time, space, temperature, anything), it is said that $\mathrm{x}(u)$ represents a stochastic process. In this case, a full characterization of the process requires more than measuring $\rho(\mathrm{x})$ at any $u$, it 
requires obtaining $\rho\left(\mathrm{x} \mid u_{1}\right), \rho\left(\mathrm{x} \mid u_{2}\right), \rho\left(\mathrm{x} \mid u_{3}\right), \ldots, \rho\left(\mathrm{x} \mid u_{n}\right)$, i.e., measuring the distribution $\rho(\mathrm{x} \mid u)$ for each and every possible value of $u$.

Obviously this is an exhausting task, almost always impossible (if $\mathrm{u}$ is continuous, there are infinite values of $u$ for which $\rho(\mathrm{x} \mid u)$ must be obtained), and often unnecessary.

\section{Sample Mean}

When it is possible to obtain a sample of $\mathrm{x}$ at a fix $u_{0}$, the sample mean is calculated by $\left\langle\mathrm{x}\left(u_{0}\right)\right\rangle$ (and other moments are analogous); the process is repeated at $u_{1}$ to calculate $\left\langle\mathrm{x}\left(u_{1}\right)\right\rangle$, etc.

But often it is impossible. For example, if $u$ is time, $N$ measurements of $\mathrm{x}\left(u_{0}\right)$ should be done simultaneously at the instant $u_{0}$; it would require $N$ identic experimental apparatuses in perfect synchrony, something no real lab has. So, it is desirable another procedure, capable of obtaining the same results of the sample mean (at least approximately).

\section{Autocorrelation and Stationary Processes}

Treating $\mathrm{x}\left(u_{1}\right), \mathrm{x}\left(u_{2}\right), \ldots, \mathrm{x}\left(u_{n}\right)$ as separate variable (but not necessarily independent), the relation between $\mathrm{x}\left(u_{i}\right)$ and $\mathrm{x}\left(u_{j}\right)$ is estimated by the autocorrelation:

$$
R_{\mathrm{x}}\left(u_{i}, u_{j}\right)=\left\langle\mathrm{x}\left(u_{i}\right) \mathrm{x}\left(u_{j}\right)\right\rangle
$$

In many situations the autocorrelation is not a function of $u_{i}$ and $u_{j}$, but of $v=u_{i}-u_{j}$, so

that $R_{\mathrm{x}}\left(u_{i}, u_{j}\right)=R_{\mathrm{x}}(v)$. Such processes are called stationary, because $R_{\mathrm{x}}(v)$ is not altered by changing $u_{i}$ for $u_{i}+v$ (a symmetry analogous to translation invariance).

\section{Moving Average}

The moving average of $\mathrm{x}(u)$ over an interval of $u$ is calculated by:

$$
\left.\langle\mathrm{x}(\tilde{u})\rangle\right|_{u_{0}} ^{u_{1}}=\frac{1}{u_{1}-u_{0}} \int_{u_{0}}^{u_{1}} \mathrm{x}\left(u^{\prime}\right) d u^{\prime}
$$


Where $\tilde{u}=\frac{u_{1}-u_{0}}{2}$.

This average can have no meaning, it might diverge, it can be very troublesome in many ways. But under the right conditions, this is a good estimative of the sample mean.

One such condition is that the process is stationary; so, defining $\mathrm{U}=u_{1}-u_{0}$ and rewriting B16:

$$
\left.\langle\mathrm{x}(u)\rangle\right|_{U}=\frac{1}{U} \int_{u-U / 2}^{u+U / 2} \mathrm{x}\left(u^{\prime}\right) d u^{\prime}
$$

Assume there are $u_{i}$ and $u_{j}$ inside the interval $\left[u_{0}, u_{1}\right]$, and two sets of $\mathrm{N}$ data, $\mathrm{x}\left(u_{i}\right)$ and $\mathrm{x}\left(u_{j}\right)$. If the variation of $\mathrm{x}(u)$ is sufficiently small inside this interval, then it is expected that $\left\langle\mathrm{x}\left(u_{i}\right)\right\rangle \approx\left\langle\mathrm{x}\left(u_{j}\right)\right\rangle$ (i.e., the sample mean of $\mathrm{x}$ in any instant inside the interval is approximately the same). Being so, it is possible to join the two data sets in one, even if they refer to different instants, and do the average over all the data; and this is the same as doing the moving average over the interval.

Back to the example where $u$ represents time, it is possible to do, with a single apparatus, many measurements of $\mathrm{x}(u)$, not simultaneous but over a very short period, and calculate the moving average. If $\mathrm{x}(u)$ does not vary abruptly in this interval, $\left.\langle\mathrm{x}(u)\rangle\right|_{U}$ is a good estimative of $\langle\mathrm{x}(u)\rangle$.

The other moments and the autocorrelation can also be calculated by the same idea of the moving average:

$$
\begin{gathered}
\left.\left\langle\mathrm{x}^{n}(u)\right\rangle\right|_{U}=\frac{1}{U} \int_{u-U / 2}^{u+U / 2} \mathrm{x}^{n}\left(u^{\prime}\right) d u^{\prime} \\
\left.R_{\mathrm{x}}(v)\right|_{U}=\lim _{U \rightarrow \infty} \frac{1}{U} \int_{u-U / 2}^{u+U / 2} \mathrm{x}\left(u^{\prime}+v\right) \mathrm{x}\left(u^{\prime}\right) d u^{\prime}
\end{gathered}
$$




\section{Wiener Spectrum}

The same way it is interesting analyzing signals in the frequency space, the spectral analysis of a stochastic process may have useful information ${ }^{27}$. However if the process is stationary, the total energy of the signal must be infinite:

$$
\begin{gathered}
\mathrm{X}(v)=\mathcal{F}_{v}[\mathrm{x}(u)] \\
E=\int_{-\infty}^{\infty}|\mathrm{x}(u)|^{2} d u=\int_{-\infty}^{\infty}|\mathrm{X}(v)|^{2} d v \rightarrow \infty
\end{gathered}
$$

This result is not wrong; for example, if $u$ is time, $|\mathrm{x}(u)|^{2}$ would be the power transmitted by the signal over time, and so a signal that lasts forever has carries infinite energy. But this result does not help analyzing the signal. So another method of looking at signals in the frequency space is needed.

Assuming the signal $\mathrm{x}(u)$ is periodic of period $T$, it can be expanded in Fourier series, and its autocorrelation as well:

$$
\begin{aligned}
& \mathrm{x}(u)=\sum_{n=-\infty}^{\infty} \mathrm{X}_{n} e^{2 \pi i n v_{0} u} \rightarrow \mathrm{X}_{n}=\frac{1}{T} \int_{-T / 2}^{T / 2} \mathrm{x}(u) e^{-2 \pi i n v_{0} u} d u \\
& R_{\mathrm{x}}(v)=\sum_{n=-\infty}^{\infty} a_{n} e^{2 \pi i n v_{0} v} \rightarrow a_{n}=\frac{1}{T} \int_{-T / 2}^{T / 2} R_{\mathrm{x}}(v) e^{-2 \pi i n v_{0} v} d v
\end{aligned}
$$

Calculating then the correlation between the coefficients $\mathrm{X}_{n}$ :

$$
\left\langle X_{n} X_{m}^{*}\right\rangle=a_{n} \delta_{n m}
$$

So, the coefficients of the $\mathrm{x}(u)$ expansion are uncorrelated to each other, and relate to the coefficients of the $R_{\mathrm{x}}(v)$ expansion. Then the Wiener spectrum is defined: 


$$
W_{\mathrm{x}}(v) \equiv \sum_{n=-\infty}^{\infty} a_{n} \delta\left(v-n v_{0}\right)=\mathcal{F}_{v}\left[R_{\mathrm{x}}(v)\right]
$$

In spite of using a periodic $\mathrm{x}(u)$ in the deduction, this definition is also valid for nonperiodic signals.

Alternatively, the divergence problem of $\mathrm{X}(v)$ can be avoided defining $\widetilde{X}(v)$, the truncated Fourier transform of the signal:

$$
\widetilde{\mathrm{X}}(v)=\widetilde{\mathcal{F}_{v}}[\mathrm{x}(u)]=\int_{-\vartheta}^{\vartheta} e^{-2 \pi i v u} \mathrm{x}(u) d u
$$

This way, $|\widetilde{\mathrm{X}}(v)|^{2}$ is the power of the component of the signal that has frequency $v$. The power spectrum is then defined:

$$
\begin{gathered}
\mathcal{W}(v) \equiv \lim _{\vartheta \rightarrow \infty}\left\langle|\widetilde{\mathrm{X}}(v)|^{2}\right\rangle \\
\mathcal{W}(v)=\lim _{\vartheta \rightarrow \infty}\left\langle\left(\int_{-\vartheta}^{\vartheta} e^{-2 \pi i v u_{i}} \times\left(u_{i}\right) d u_{i}\right)\left(\int_{-\vartheta}^{\vartheta} e^{2 \pi i v u_{j}} \mathrm{X}^{*}\left(u_{j}\right) d u_{j}\right)\right\rangle \\
\mathcal{W}(v)=\lim _{\vartheta \rightarrow \infty}\left\langle\int_{-\vartheta}^{\vartheta} d u_{j} \int_{-\vartheta}^{\vartheta} d u_{i} e^{-2 \pi i v\left(u_{i}-u_{j}\right)} \mathrm{x}\left(u_{i}\right) \mathrm{x}\left(u_{j}\right)\right\rangle
\end{gathered}
$$

In the limit $\vartheta \rightarrow \infty$, the truncated transform $\widetilde{\mathcal{F}_{v}}$ equals the ordinary transform $\mathcal{F}_{v}$; so:

$$
\mathcal{W}(v)=\mathcal{F}_{v}\left[\left\langle\mathrm{x}\left(u_{i}\right) \mathrm{x}\left(u_{j}\right)\right\rangle\right]=W_{\mathrm{x}}(v)
$$

This shows the Wiener spectrum represents the distribution of the power of the signal over each frequency, and has two equivalent definitions:

$$
W_{\mathrm{x}}(v)=\lim _{\vartheta \rightarrow \infty}\left\langle\left|\widetilde{\mathcal{F}_{v}}[\mathrm{x}(u)]\right|^{2}\right\rangle=\mathcal{F}_{v}\left[R_{\mathrm{x}}(v)\right]
$$




\section{Gaussian Distribution}

Also called normal distribution, the $n$-dimensional Gaussian has the form:

$$
\rho(\mathbf{x})=\frac{1}{\sqrt{(2 \pi)^{n} \operatorname{det}(V)}} e^{-\frac{(\mathbf{x}-\overline{\mathbf{x}}) \cdot V^{-1} \cdot(\mathbf{x}-\overline{\mathbf{x}})^{\prime}}{2}}
$$

Where $(\mathbf{x}-\overline{\mathbf{x}})^{\prime}$ denotes a transpose vector, and $\overline{\mathbf{x}}$ and $V$ are the vector of means and covariance matrix of the $n$-dimensional variable $\mathbf{x}=\left(\mathrm{x}_{1}, \mathrm{x}_{2}, \ldots, \mathrm{x}_{n}\right)$ :

$$
V=\left(\begin{array}{ccc}
\left\langle\left(\mathrm{x}_{1}-\overline{\mathrm{x}_{1}}\right)\left(\mathrm{x}_{1}-\overline{\mathrm{x}_{1}}\right)\right\rangle & \cdots & \left\langle\left(\mathrm{x}_{n}-\overline{\mathrm{x}_{n}}\right)\left(\mathrm{x}_{1}-\overline{\mathrm{x}_{1}}\right)\right\rangle \\
\vdots & \ddots & \vdots \\
\left\langle\left(\mathrm{x}_{1}-\overline{\mathrm{x}_{1}}\right)\left(\mathrm{x}_{n}-\overline{\mathrm{x}_{n}}\right)\right\rangle & \cdots & \left\langle\left(\mathrm{x}_{n}-\overline{\mathrm{x}_{n}}\right)\left(\mathrm{x}_{n}-\overline{\mathrm{x}_{n}}\right)\right\rangle
\end{array}\right)
$$

In particular, in one dimension:

$$
\rho(\mathrm{x})=\frac{1}{\sqrt{2 \pi} \sigma_{\mathrm{x}}} e^{-\frac{1}{2}\left(\frac{\mathrm{x}-\overline{\mathrm{x}}}{\sigma_{\mathrm{x}}}\right)^{2}}
$$

The Gaussian distribution is probably the most used distribution in all areas of science, for 2 reasons.

According to the principle of maximum entropy, knowing only mean and variance of a dada set, the Gaussian distribution is the one that best represents the data (it is the one that maximizes entropy). As often the mean and variance are all information available, the normal distribution becomes the best starting point.

Besides, the Central Limit Theorem shows that the distribution of a sum of $N$ random variables (each with its own distribution, no restrictions imposed) tends to a Gaussian as $N$ tends to infinity. This means that, when the measured variable is a function of a large number of other inaccessible random variables, the Gaussian is a good approximation.

\section{Poisson Distribution}

The Poisson distribution describes the probability $\operatorname{Pr}(\mathrm{K} \mid T)$ of an event occurring $\mathrm{K}$ times in a given interval of time $T$ (or space, or any parameter). The following suppositions are made: 
1) the number of occurrences of the event in the interval $T$ is statistically independent of the occurrence in any other non-overlapping interval $\mathrm{T}^{\prime}$;

2) the probability of 1 occurrence in an infinitesimal interval $\Delta T$ is proportional to $\Delta T$ (i.e., $\operatorname{Pr}(1 \mid \Delta T)=\alpha \Delta T)$;

3) the probability of more than 1 occurrence in $\Delta T$ is zero $(\operatorname{Pr}(\mathrm{K}>1 \mid \Delta T)=0)$.

From these assumptions, the following formula is deduced:

$$
\operatorname{Pr}(\mathrm{K} \mid T)=\frac{(\alpha T)^{\mathrm{K}}}{\mathrm{K} !} e^{-\alpha T}
$$

Where $\alpha$ is the mean number of occurrences per unit of time. Defining $(\alpha T) \equiv \overline{\mathrm{K}}$ :

$$
\operatorname{Pr}(\mathrm{K})=\frac{\overline{\mathrm{K}}^{\mathrm{K}}}{\mathrm{K} !} e^{-\overline{\mathrm{K}}}
$$

As the notation suggests, $\overline{\mathrm{K}}$ is the mean of the Poisson distribution. Being the mean the only parameter of this distribution, all other moments are written as functions of the mean. In particular:

$$
\sigma_{\mathrm{K}}^{2}=\overline{\mathrm{K}}
$$

For a large $\overline{\mathrm{K}}$, the term $\frac{\overline{\mathrm{K}}^{\mathrm{K}}}{\mathrm{K} !}$ tends to cause numeric problems; in this case the following approximation is useful:

$$
\operatorname{Pr}(\mathrm{K}) \approx \frac{1}{\sqrt{2 \pi \overline{\mathrm{K}}}} e^{-\frac{(\mathrm{K}-\overline{\mathrm{K}})^{2}}{2 \overline{\mathrm{K}}}}
$$

For a more complete description of a phenomena that follows the Poisson statistics, it would also be necessary quantifying the instants (or positions) of each particular occurrence of the event (and these are also stochastic variables). By the first supposition of the Poisson distribution, the probability that the $j$-th occurrence happens in the instant $t_{j}$ is: 


$$
\operatorname{Pr}\left(t_{j}\right)=\left\{\begin{array}{cc}
T^{-1} & \text { if } 0<t_{j}<T \\
0 & \text { else }
\end{array}\right.
$$

So, in the observed interval, an event can occur at any instant with equal probability.

The Poisson distribution describes a great variety of phenomena in all areas; in particular, photon sources (radioactive samples, X-ray tubes, light bulbs, etc.) emit according to this distribution (the emission of one photon can occur at any instant and in any direction). A Poisson process would then be described by the sum of delta impulses at the instant of each occurrence:

$$
\mathrm{z}(t)=\sum_{j=1}^{\mathrm{K}} \delta\left(t-t_{j}\right)
$$

Mean and autocorrelation of $\mathrm{z}$ can then be calculated:

$$
\begin{gathered}
\langle\mathrm{z}(t)\rangle=\sum_{\mathrm{K}=0}^{\infty} \operatorname{Pr}(\mathrm{K}) \prod_{i=1}^{\mathrm{K}}\left(\int_{0}^{T} \operatorname{Pr}\left(t_{i}\right) d t_{i}\right) \sum_{j=1}^{\mathrm{K}} \delta\left(t-t_{j}\right)=\frac{\overline{\mathrm{K}}}{T}=\alpha \\
R_{z}(\tau)=\langle\mathrm{z}(t) \mathrm{z}(t+\tau)\rangle=\alpha \delta(\tau)+\alpha^{2}
\end{gathered}
$$

\section{Student's t-distribution}

Consider a random variable $\mathrm{x}$, described by the Gaussian distribution with mean and standard deviation true values ( $\mu$ and $\sigma$ ) equal zero and one respectively. Taking an infinite number of data points, the estimates of mean and standard deviation of $\mathrm{x}(\overline{\mathrm{x}}$ and $\hat{\sigma})$ tend to $\mu$ and $\sigma$. However, no experiment measures infinite data points. So, it is necessary to know the distributions not of $\mathrm{x}$, but of the $N$ measurements of $\mathrm{x}$ (where $N$ is finite and often low). The t-distribution is the one that does it, and is used to perform tests of hypothesis about the estimated value $\overline{\mathrm{x}}$ (taking $\hat{\sigma}$ into account).

The t-distribution is: 


$$
\rho_{v}(t)=\frac{\Gamma\left(\frac{v+1}{2}\right)}{\sqrt{v \pi} \Gamma\left(\frac{v}{2}\right)}\left(1+\frac{t^{2}}{v}\right)^{-\frac{v+1}{2}}
$$

Where $v=N-1$ is the number of degrees of freedom and $\Gamma$ is the Gamma function. This distribution is used, among other situations, for the t-test. The probability that $t$ lies between 2 values $-t_{\text {crit }}$ and $t_{c r i t}$ is:

$$
\operatorname{Pr}\left[-t_{\text {crit }} \leq t \leq t_{\text {crit }}\right]=\int_{-t}^{t} \rho_{v}(t) d t=1-p
$$

This $t_{\text {crit }}$ value is often chosen so that $\operatorname{Pr}\left[-t_{\text {crit }} \leq t \leq t_{\text {crit }}\right]$ equals a "standardized" probability $1-p$ (this $p$ is often referred as the $p$-value). Common values of $p$ are 0.05 (the "2 sigma interval", or "95\% interval") or 0.01 (the "3 sigma interval", or "99\% interval"), but this value can be chosen according to the situation. There are many tables of $t_{\text {crit }}$ values for each $v$ at some standard p-values.

Then assume the hypothesis $H$ that the true value $\mu$ (which is unknown) is $\mathrm{x}_{0}$, a chosen value, and calculate $t$ :

$$
t=\frac{\overline{\mathrm{x}}-\mathrm{x}_{0}}{\hat{\sigma} / \sqrt{N}}
$$

This quantity is interpreted as how many (estimated) mean standard deviations the (estimated) mean is away from the (supposed) true mean. If $-t_{c r i t} \leq t \leq t_{c r i t}$, then the hypothesis $H: \mu=\mathrm{x}_{0}$ cannot be rejected. Otherwise, it can be said with enough certainty that the hypothesis is false.

A variation of this test is used to compare 2 mean values $\overline{x_{1}}$ and $\overline{x_{2}}$ obtained independently, with their corresponding estimated standard deviations $\sigma_{1}$ and $\sigma_{2}$ and (generally) different number of data points $n_{1}$ and $n_{2}$ (and degrees of freedom). First it is calculated the combined standard deviation:

$$
\sigma^{2}=\frac{\sigma_{1}^{2}\left(n_{1}-1\right)+\sigma_{2}^{2}\left(n_{2}-1\right)}{n_{1}+n_{2}-2}
$$


Then the $t$ value is calculated by:

$$
t=\frac{\overline{x_{1}}-\overline{x_{2}}}{\sigma \sqrt{\frac{1}{n_{1}}+\frac{1}{n_{2}}}}
$$

From there, $t$ is compared to $t_{\text {crit }}$ in the same way (taking care that the number of degrees of freedom to be used is $n_{1}+n_{2}-2$ ).

\section{Chi-squared and Reduced Chi-squared Distribution}

If $\mathrm{x}$ is a normal random variable of zero mean and standard deviation 1 , measured $N$ times $\left(\mathrm{x}_{1}, \mathrm{x}_{2}, \ldots \mathrm{x}_{N}\right)$, the quantity $Q=\sum_{i=1}^{N} \mathrm{x}_{i}^{2}$ follows the chi-squared distribution $\chi^{2}(Q ; v)$ of $v$ degrees of freedom:

$$
\chi^{2}(Q ; v)= \begin{cases}\frac{Q^{\frac{v}{2}-1} e^{-\frac{Q}{2}}}{2^{\frac{\nu \Gamma\left(\frac{v}{2}\right)}{2}}} & Q>0 \\ 0 & Q \leq 0\end{cases}
$$

For a normal variable with any other mean and/or standard deviation, the $Q$ quantity is:

$$
Q=\mathbf{X}^{\prime} \mathbf{V}^{-1} \mathbf{X}
$$

Where $\mathbf{X}=(\mathrm{x} 1, \mathrm{x} 2, \ldots \mathrm{x} N)$ and $\mathbf{V}$ is the variance matrix of these measurements.

This distribution is more practical in its reduced form $\chi_{\text {red }}^{2}\left(Q_{\text {red }} ; v\right)$ :

$$
\begin{gathered}
Q_{\text {red }}=\frac{Q}{v} \\
\chi_{\text {red }}^{2}\left(Q_{\text {red }} ; v\right)=v \chi^{2}\left(v Q_{\text {red }} ; v\right)
\end{gathered}
$$

Notably, the quotient of the estimated variance and the true variance of a normal variable follows a reduced chi-squared distribution where $v=N-1$. 
The reduced chi-squared distribution is used to evaluate the goodness of a fit. Consider a measured function $\mathrm{y}\left(x_{i}\right)$ with its uncertainty $\sigma_{\mathrm{y}(x i)}$, and a fitted function $\hat{\mathrm{y}}\left(x_{i}\right)$ with $\mu$ parameters $(i=1,2, \ldots N)$. The difference $\mathrm{y}\left(x_{i}\right)-\hat{\mathrm{y}}\left(x_{i}\right)$ is assumed normal (often a good assumption, from the Central Limit Theorem). The $Q_{\text {red }}$ quantity is calculated:

$$
Q_{r e d}=\frac{(\mathbf{y}-\hat{\mathbf{y}})^{\prime} \mathbf{V}^{-1}(\mathbf{y}-\hat{\mathbf{y}})}{N-\mu}=\frac{1}{N-\mu} \sum_{i}\left(\frac{\mathrm{y}\left(x_{i}\right)-\hat{\mathrm{y}}\left(x_{i}\right)}{\sigma_{\mathrm{y}(x i)}}\right)^{2}
$$

The expected value of $Q_{r e d}$ is 1 ; if $Q_{r e d} \ll 1$, either the uncertainty of the data points are overestimated or the points are overfitted ${ }^{80}$; if $Q_{\text {red }} \gg 1$, either the uncertainties are underestimated or the fitting function is wrong. A good fit will have $Q_{\text {red }}$ close to 1 , but how much "close" means depends on the number of degrees-of-freedom.

Having the calculated $Q_{r e d}$, a test of hypothesis is conducted (a chi-square test); a p-value (probability of considering the fit bad and rejecting the result) is chosen, a critical value $Q_{\text {red,crit }}$ is obtained compared to the calculated $Q_{r e d}$.

Since the reduced chi-squared distribution is asymmetric, this test of hypothesis is often one-tailed (it rejects values only at one extreme of the curve), or done once for each tail. For example, if there is enough confidence that the uncertainties were not overestimated and there is no overfitting, only the right tail (high $Q_{r e d}$ ) must be rejected. In this case:

$$
\int_{0}^{Q_{\text {red,Crit }}} \chi_{\text {red }}^{2}\left(Q_{\text {red }} ; v\right) d Q_{\text {red }}=1-p
$$

Then, if $Q_{\text {red }}<Q_{\text {red,Crit }}$, the fit is accepted as good. If not, it is rejected.

\section{Fisher's F-distribution}

The Fisher F-distribution is used to test hypotheses about the variances (the F-test) in much the same way the t-distribution is used to test hypotheses about mean values. Suppose there are 2 measurements of the variance of a quantity, $\sigma_{1}^{2}$ and $\sigma_{2}^{2}$, with $v_{1}$ and $v_{2}$ degrees of freedom respectively. To compare them, the $F$ value is calculated: 


$$
F=\frac{\sigma_{1}^{2}}{\sigma_{2}^{2}}
$$

This quantity follows the Fisher distribution:

$$
\rho\left(F, v_{1}, v_{2}\right)=\frac{\sqrt{\frac{\left(v_{1} F\right)^{v_{1}} v_{2} v_{2}}{\left(v_{1} F+v_{2}\right)^{v_{1}+v_{2}}}}}{F \cdot B\left(\frac{v_{1}}{2}, \frac{v_{2}}{2}\right)}
$$

Where $B\left(\frac{v_{1}}{2}, \frac{v_{2}}{2}\right)$ is the Beta function. The mean and variance of $F$ are:

$$
\begin{gathered}
\langle F\rangle=\frac{v_{2}}{v_{2}-2} \\
\sigma_{F}^{2}=\frac{2 v_{2}^{2}\left(v_{1}+v_{2}-2\right)}{v_{1}\left(v_{2}-2\right)^{2}\left(v_{2}-4\right)}
\end{gathered}
$$

The probability of $F$ falling inside an interval $\left[F_{-}, F_{+}\right]$is:

$$
\operatorname{Pr}\left[F_{-} \leq F \leq F_{+}\right]=\int_{F_{-}}^{F_{+}} \rho\left(F, v_{1}, v_{2}\right) d F=1-p
$$

The values $F_{-}$and $F_{+}$are, analogously to the t-test, defined by the choice of p-value. Then assume the hypothesis that the variances being compared $\sigma_{1}^{2}$ and $\sigma_{2}^{2}$ are equal $(H: F=1)$. If $F$ is inside this interval, the hypothesis cannot be rejected ( $\sigma_{1}^{2}$ and $\sigma_{2}^{2}$ are most likely equal, indeed). If not, it can be said with some certainty that they are different.

\section{Binomial Distribution}

Consider $N$ independent experiments are done with 2 possible mutually exclusive outcomes (yes or no, zero or one, positive or negative, success or failure, and so on). One outcome has probability $p$ of happening, and the other, consequently, $1-p$. The 
probability of obtaining n "successes" (n times one of the outcomes, $N-\mathrm{n}$ the other) is given by the binomial distribution:

$$
\rho(\mathrm{n} \mid N, p)=\frac{N !}{\mathrm{n} !(N-\mathrm{n}) !} p^{\mathrm{n}}(1-p)^{N-\mathrm{n}}
$$

This distribution has average and variance of:

$$
\begin{gathered}
\langle\mathrm{n}\rangle=N p \\
\sigma_{\mathrm{n}}^{2}=N p(1-p)
\end{gathered}
$$

This distribution has many uses, one of them is in statistical tests like the t-test, chi-square test and F-test presented earlier. In such tests, for a given p-value, there is a probability $p$ that the quantity calculated $(t, Q, F$, etc) falls outside the acceptance interval (a probability $p$ of rejecting the result). If quantity is measured $N$ times independently, the number of rejections $\mathrm{n}$ (or acceptances) follows a binomial distribution.

\section{Test of Hypothesis and Error Types}

Consider a binary hypothesis $H$ (it can be true or false). The hypothesis that $H$ is wrong is called the null hypothesis, usually denoted $H_{0}$. By statistical convention, $H$ is usually stated in a way that makes $H_{0}$ a negation (e.g.: $H$ : A is different of B.). An experiment is then performed to assess its validity, and the experiment can accept or reject $H_{0}$.

If $H_{0}$ was true and the experiment accepted it, the experiment worked as intended, and this result is a called true negative ( $H$ is indeed wrong). Similarly, if $H_{0}$ was false and the experiment rejected it, the experiment also worked as intended and this result is called a true positive ( $H$ is right).

However, there is also the possibility that the experiment will reject $H_{0}$ when it is true. This is called a false positive, or a type I error. The opposite, accepting $H_{0}$ when it is false, is called a false negative, or a type II error.

This may seem a very restrict case, usable only for binary hypotheses and binary experiments, but almost any situation may be reduced to this. Suppose there is a 
stochastic variable $\mathrm{x}$ which follows a distribution $\rho(\mathrm{x})$ (any distribution). A p-value is chosen, defining a range of $\mathrm{x}$ values. The variable $\mathrm{x}$ may be continuous (having infinite possible values), but the hypothesis made about $\mathrm{x}$ is binary: either $\mathrm{x}$ is within the defined range (with probability $1-p$ ) or it is outside (with probability $p$ ). Similarly, the experiment that measures $\mathrm{x}$ is also binary: either it measures a value within the range (accepting the hypothesis) or outside (rejecting). 


\section{APPENDIX C: NOISE FILTERING}

\section{Stochastic Noise}

Given a linear filter of known $P S F(\boldsymbol{r})$, and assuming the noise at the input signal $\mathrm{x}_{i n}(\boldsymbol{r})$ is a stationary process:

$$
\begin{array}{lll}
\mathrm{x}_{\text {out }}(\boldsymbol{r})=P S F(\boldsymbol{r}) * \mathrm{x}_{\text {in }}(\boldsymbol{r}) & P(\boldsymbol{f})=\mathcal{F}_{\boldsymbol{k}}[P S F(\boldsymbol{r})] & \mathrm{C} 1 \\
R_{\text {in }}(\xi)=\left\langle\mathrm{x}_{\text {in }}(\boldsymbol{r}) \mathrm{x}_{\text {in }}(\boldsymbol{r}+\xi)\right\rangle & W_{\text {in }}(\boldsymbol{f})=\mathcal{F}_{\boldsymbol{k}}\left[R_{\text {in }}(\xi)\right] & \mathrm{C} 2
\end{array}
$$

With these relations, the autocorrelation and Wiener spectra of the output signal can be calculated:

$$
\begin{gathered}
R_{\text {out }}(\xi)=\left\langle\mathrm{x}_{\text {out }}(\boldsymbol{r}) \mathrm{x}_{\text {out }}(\boldsymbol{r}+\xi)\right\rangle=\left\langle\left(P S F(\boldsymbol{r}) * \mathrm{x}_{\text {in }}(\boldsymbol{r})\right) \cdot\left(P S F(\boldsymbol{r}+\xi) * \mathrm{x}_{\text {in }}(\boldsymbol{r}+\xi)\right)\right\rangle \\
W_{\text {out }}(\boldsymbol{f})=\mathcal{F}_{\boldsymbol{k}}\left[\left\langle\left(P S F(\boldsymbol{r}) * \mathrm{x}_{\text {in }}(\boldsymbol{r})\right) \cdot\left(P S F(\boldsymbol{r}+\xi) * \mathrm{x}_{\text {in }}(\boldsymbol{r}+\xi)\right)\right\rangle\right]
\end{gathered}
$$

Simplifying:

$$
W_{\text {out }}(\boldsymbol{f})=|P(\boldsymbol{f})|^{2} W_{\text {in }}(\boldsymbol{f})
$$

As often mean and variance are enough:

$$
\begin{gathered}
\overline{\mathrm{x}}_{\text {out }}=\left\langle\mathrm{x}_{\text {out }}(\boldsymbol{r})\right\rangle=P S F(\boldsymbol{r}) *\left\langle\mathrm{x}_{\text {in }}(\boldsymbol{r})\right\rangle \\
\overline{\mathrm{x}}_{\text {out }}=P(\mathbf{0}) \cdot \overline{\mathrm{x}}_{\text {in }} \\
\sigma_{\text {out }}^{2}=\left\langle\mathrm{x}_{\text {out }}^{2}(\boldsymbol{r})\right\rangle-\left\langle\mathrm{x}_{\text {out }}(\boldsymbol{r})\right\rangle^{2}=R_{\text {out }}(\mathbf{0})-\overline{\mathrm{x}}_{\text {out }}^{2} \\
\sigma_{\text {out }}^{2}=\int_{\Omega} W_{\text {out }}(\boldsymbol{f}) d \boldsymbol{f}-\overline{\mathrm{x}}_{\text {out }}^{2}
\end{gathered}
$$




\section{Optimized Filters}

Having the linear systems theory and the statistic tools needed to characterize stochastic signals (like noise), an optimized filter can be designed to return the "best" possible output. What is this filter depends of how one defines "best", what assumptions are made about the input, etc. Different approaches to these questions will result in different filters, and there are many such examples in the literature; here only one will be exemplified, the Wiener-Helstrom filter.

Consider a signal $s(\boldsymbol{r})$, altered by a known filter $h(\boldsymbol{r})$ and added of a stationary noise $\mathrm{x}(\boldsymbol{r})$ (independent of the signal):

$$
\mathrm{s}_{i n}(\boldsymbol{r})=\mathrm{s}(\boldsymbol{r}) * h(\boldsymbol{r})+\mathrm{x}(\boldsymbol{r})
$$

The Wiener-Helstrom filter, denoted $p(\boldsymbol{r})$ (or $P(\boldsymbol{f})$, in the frequency space), intends on extracting the best estimative of $s(\boldsymbol{r})$, denoted $\widehat{\mathrm{s}}(\boldsymbol{r})$, from $s_{i n}(\boldsymbol{r})$ :

$$
\widehat{\mathrm{s}}(\boldsymbol{r})=\mathrm{s}_{i n}(\boldsymbol{r}) * p(\boldsymbol{r})
$$

It is also assumed that the autocorrelations $R_{\mathrm{S}}(\boldsymbol{r})$ and $R_{\mathrm{x}}(\boldsymbol{r})$ of $\mathrm{s}(\boldsymbol{r})$ and $\mathrm{x}(\boldsymbol{r})$ are known. "Best estimative" of $s(\boldsymbol{r})$ is defined as the one that minimizes the mean square error $\left\langle|\hat{s}(\boldsymbol{r})-s(\boldsymbol{r})|^{2}\right\rangle$. The orthogonality principle says the filter that minimizes this quantity also makes the errors orthogonal to the data, i.e.:

$$
\left\langle(\hat{\mathrm{s}}(\boldsymbol{r})-\mathrm{s}(\boldsymbol{r})) \mathrm{s}_{i n}(\boldsymbol{r})\right\rangle=0
$$

Substituting C6 and C7 in C8:

$$
\begin{gathered}
\langle((\mathrm{s}(\boldsymbol{r}) * h(\boldsymbol{r})+\mathrm{x}(\boldsymbol{r})) * p(\boldsymbol{r})-\mathrm{s}(\boldsymbol{r}))(\mathrm{s}(\boldsymbol{r}) * h(\boldsymbol{r})+\mathrm{x}(\boldsymbol{r}))\rangle=0 \\
R_{\mathrm{s}}(\boldsymbol{r}) *(h(\boldsymbol{r}) * h(\boldsymbol{r}) * p(\boldsymbol{r})-h(\boldsymbol{r}))+R_{\mathrm{x}}(\boldsymbol{r}) * p(\boldsymbol{r})=0
\end{gathered}
$$

In terms of the Wiener spectra $W_{\mathrm{s}}(\boldsymbol{f})$ and $W_{\mathrm{x}}(\boldsymbol{f})$ of $\mathrm{s}(\boldsymbol{r})$ and $\mathrm{x}(\boldsymbol{r})$, respectively: 


$$
\begin{gathered}
W_{\mathrm{s}}(\boldsymbol{f})\left(\left|H^{2}(\boldsymbol{f})\right| \cdot P(\boldsymbol{f})-H^{*}(\boldsymbol{f})\right)+W_{\mathrm{x}}(\boldsymbol{f}) P(\boldsymbol{f})=0 \\
P(\boldsymbol{f})=\frac{H^{*}(\boldsymbol{f})}{\left|H^{2}(\boldsymbol{f})\right|+\frac{W_{\mathrm{x}}(\boldsymbol{f})}{W_{\mathrm{s}}(\boldsymbol{f})}}
\end{gathered}
$$

What should be learned from this example is that designing optimized filters is no trivial task. Everything depends on what is defined as "best output" (in this example, equation C8) and what one knows (or supposes as known) about the input (in here, equation C6). For every case, the optimum filter is different, and may not be possible to implement at all.

\section{Windowing}

Optimized filters like the Wiener-Helstrom are generally more useful for didactic purposes than practical applications. In the above example, it is supposed $h(\boldsymbol{r})$ is known, and also the autocorrelations $R_{s}(\boldsymbol{r})$ and $R_{\mathrm{x}}(\boldsymbol{r})$; these functions are not always known.

In practice, a window function $J(f)$ (sometimes called apodizing function) is chosen; this function, applied to the $M T F$ of the system, is used to select only the frequencies in which the signal dominates, eliminating the frequencies that contain mostly noise.

For this function to fulfill its purpose, it must be valued 1 at the frequency zero (it must keep the normalization of the $M T F$ ) and be valued zero outside the interesting range of frequencies:

$$
\begin{gathered}
J(\mathbf{0})=1 \\
J(\boldsymbol{f})=0 \text { if }\left|f_{i}\right|>f_{i, \max } \text { for any } i=1,2 \ldots n
\end{gathered}
$$

In spite of these restrictions, there are still infinite possible choices of $J(f)$; the simplest of all is the rectangular function (one-dimensional, for simplicity);

$$
J(f)=\operatorname{rect}(a f)=\left\{\begin{array}{l}
1 \text { if }|x| \leq 1 / 2 a \\
0 \text { if }|x|>1 / 2 a
\end{array}\right.
$$


The Hamming windows, which combine cosine functions, are also much used, as they are smoother than the rectangular window; an example of such window is:

$$
J(f)=\frac{\operatorname{rect}(a f) \cdot(1+\cos (2 \pi a x))}{2}
$$

A last example, the sinc filter, or Shep-Logan window:

$$
J(f)=\operatorname{rect}(a f) \cdot \operatorname{sinc}(2 a f)
$$




\section{APPENDIX D: ADDITIONAL DETAILS}

This appendix contains any details, about any section of this work, that were deemed unfit to be included in the main text.

\section{Interpolation}

In many subsections of Chapter 3, it is said that linear interpolation was used to calculate a value. This section details the interpolation algorithm used.

Given 2 points, $\left(x_{1}, y_{1}, \sigma_{1}\right)$ and $\left(x_{2}, y_{2}, \sigma_{2}\right)$, the function $f(x)$ that linearly interpolates them is:

$$
f(x)=\frac{y_{2}-y_{1}}{x_{2}-x_{1}}\left(x-x_{1}\right)+y_{1}
$$

At the fixed desired value $x_{m}$, the corresponding values $y_{m}$ and $\sigma_{y m}$ are:

$$
\begin{gathered}
y_{m}=\frac{y_{2}-y_{1}}{x_{2}-x_{1}}\left(x_{m}-x_{1}\right)+y_{1} \\
\sigma_{y m}=\sqrt{\left(\frac{x_{m}-x_{1}}{x_{2}-x_{1}}\right)^{2} \sigma_{2}^{2}+\left(1-\frac{x_{m}-x_{1}}{x_{2}-x_{1}}\right)^{2} \sigma_{1}^{2}}
\end{gathered}
$$

Alternatively, at a fixed desired value $y_{m}$, the corresponding values $x_{m}$ and $\sigma_{x m}$ are:

$$
\begin{gathered}
x_{m}=\frac{x_{2}-x_{1}}{y_{2}-y_{1}}\left(y_{m}-y_{1}\right)+x_{1} \\
\sigma_{x m}=\left(\frac{x_{2}-x_{1}}{y_{2}-y_{1}}\right) \sqrt{\left(\frac{y_{m}-y_{1}}{y_{2}-y_{1}}\right)^{2} \sigma_{2}^{2}+\left(1-\frac{y_{m}-y_{1}}{y_{2}-y_{1}}\right)^{2} \sigma_{1}^{2}}
\end{gathered}
$$

Particularly in the section 3.3.6, the interpolation of the cutoff frequency $f_{c}$ is done by defining $y_{1}=\operatorname{MTF}\left(f_{1}\right)-2 \sigma_{M T F 1}$ and $y_{2}=\operatorname{MTF}\left(f_{2}\right)-2 \sigma_{M T F 2}$, then interpolating the point $y_{c}=\operatorname{MTF}\left(f_{c}\right)-2 \sigma_{M T F C}=0$. 


\section{Slice Thickness $2 \%$ Uncertainty}

In section 3.3.3, the algorithm for the calculation of the slice thickness is explained. It is mentioned that the slice thickness uncertainty is calculated combining the standard deviation of the mean with a second source of uncertainty: the image noise. This source of uncertainty was evaluated to be around $2 \%$ of the obtained slice thickness value, so this value was built into the software. This section intends to justify this choice for a fixed value over other options, and explain how it was obtained.

Since the noise is the source of uncertainty being evaluated, at first thought it would be reasonable to use the noise assessed in the noise logical module (see section 3.3.7) to evaluate this uncertainty. However, if the CTP401 logical module required input from the noise module output, then it would be obligatory to execute the noise module first, and it would be pointless to divide the software into modules since they would be no longer independent. It would also make the software slightly less user-friendly.

Another possibility would be to build into the CTP401 logical module an algorithm that assesses noise. This way, each module of the software would still be independent. However, there would be a redundancy in the code, since noise is already assessed in the noise module. It was considered unnecessary, so the third option was chosen: a fixed value for this source of uncertainty.

To determine this fix value, 5 protocols with varied nominal slice thicknesses were selected. In each one, the noise was measured manually (taking the standard deviation of a uniform ROI). The slice thickness was also measured manually by the same procedure described in 3.3.3, but for only one ramp each protocol. The measured noise was considered to be the uncertainty of every point of the profile traced to measure the slice thickness. This uncertainty was then propagated to the final slice thickness, and the relative uncertainty was obtained.

The protocols used and the relative uncertainties obtained are given in Table 5, as well as the average relative uncertainty from all these protocols: $2.2(5) \%$. This value was rounded to $2 \%$ and built into the software. 
Table 5: Protocols used to quantify the noise influence on the slice thickness uncertainty. The last column shows the relative uncertainties obtained for the slice thickness, and the last line shows the average of all of them.

\begin{tabular}{|c|c|c|c|}
\hline Protocol & CT Scanner & Nominal Slice Thickness & Relative Uncertainty (\%) \\
\hline 1 & Aquillion & 0.5 & 4.0 \\
\hline 6 & Brilliance16 & 1.5 & 1.7 \\
\hline 10 & Brilliance 40 & 3.0 & 2.7 \\
\hline 11 & BrillianceBigBore & 1.5 & 1.6 \\
\hline \multirow[t]{2}{*}{17} & Discovery 750 & 1.25 & 1.1 \\
\hline & & Averag & $2.2(5)$ \\
\hline
\end{tabular}

\section{Image Pairs for NPS Calculation}

As explained in section 3.3.7, the algorithm for the NPS requires at least one pair of images representing the same uniform region of the phantom. This section explains how these image pairs are determined.

Initially, all images of the CTP468 module are in the "noise" list. They are all acquired with the same protocol, but they are not separated in different acquisitions (it is not needed).

For a list of $K$ images, the algorithm creates a K-by-K identity matrix, where the $k$-th line and column represent the $k$-th image $(k=1,2, \ldots, K)$. In this matrix, " 1 " means that the image in the line is acquired at the same table position of the image in the row (therefore it is a possible image pair), and " 0 " means it is not.

The algorithm then opens the DICOM header of all images and finds the table position of each one. If the $j$-th and $l$-th images were acquired at the same table position, the value at the $j$-th line and $l$-th column (and vice-versa) is set as " 1 ". After completing this step, all possible image pairs are defined, and the software needs to choose which pairs to use in the NPS calculation.

Since this matrix is symmetric, the values below the main diagonal are ignored (they are repetitions of the values above the main diagonal). The main diagonal is also not important, since it represents the comparison of an image with itself. In other words, only the values above the main diagonal are considered. If at least one of these remaining values is " 1 ", there are image pairs, therefore the NPS can be calculated. If not, only the image noise is calculated. 
Then, the software selects the pairs to be used in a manner to avoid repetition of images. Suppose the $j$-th and $l$-th images form a pair, but the $j$-th and $n$-th images also form a pair. It is not possible to use both pairs, because in this case the $j$-th image would be used twice, creating false information in the NPS calculations. Then, if the software decides to use the $j$-th with the $l$-th image, it sets the whole $j$-th and $l$-th lines and columns of the matrix as " 0 ", preventing repetition of any of these two images.

\section{2-Dimensional Noise Power Spectrum}

Section 3.3.7 mentions that the software saves both the 2D NPS and the radial NPS. However, section 4.1.4 only presents the radial NPS, as it is the only one needed to perform the validation. This section presents the 2D NPS functions for the same protocols of the radial NPS presented before.

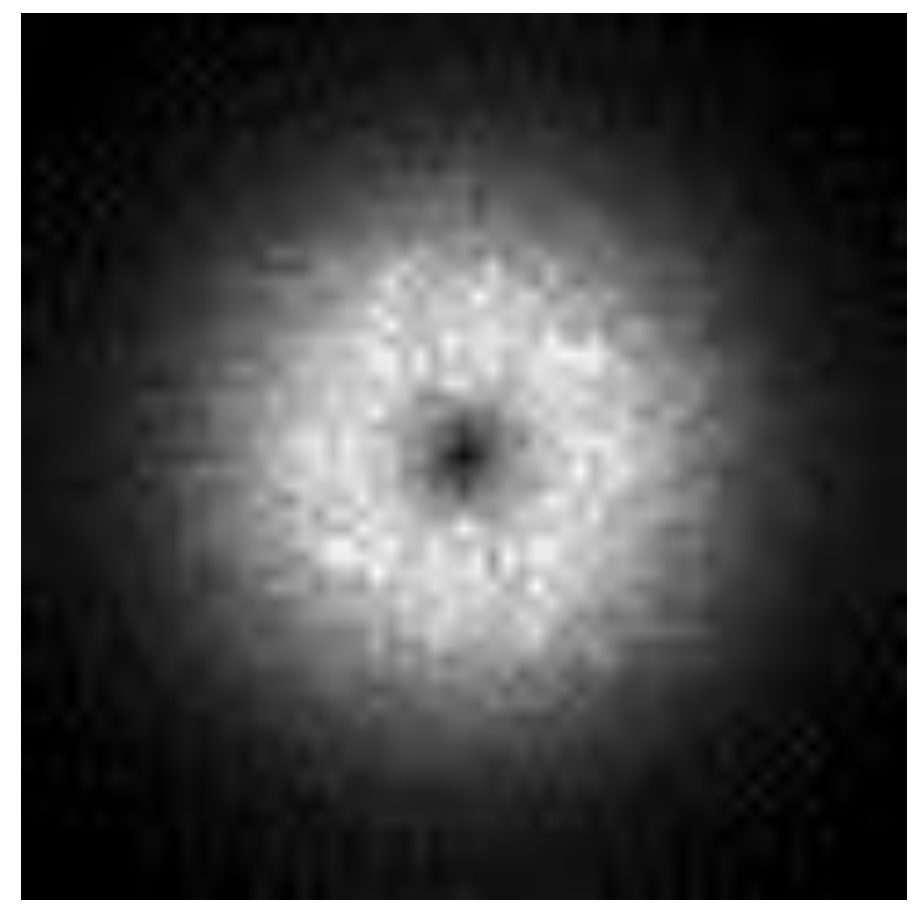

Figure 49: 2D NPS measured at protocol 2 (Toshiba Aquillion, axial mode, 1.0mm slice thickness, 240mm FOV). 


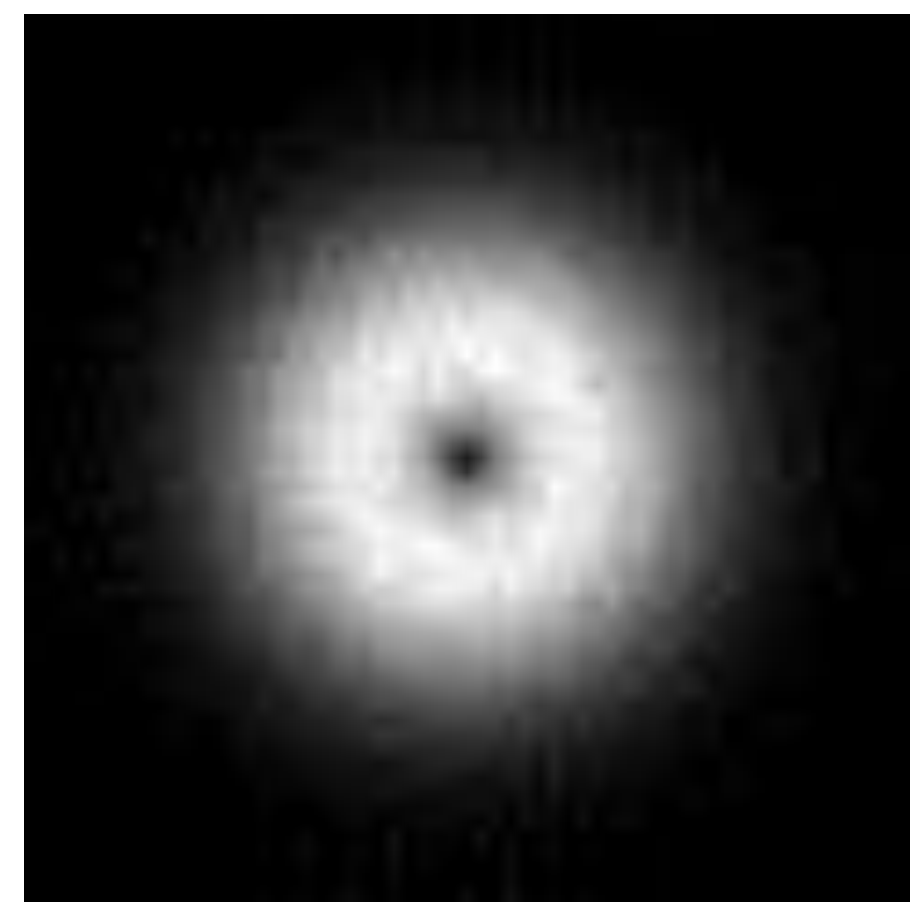

Figure 50: 2D NPS measured at protocol 6 (Phillips Brilliance16, axial mode, 1.5mm slice thickness, 250mm FOV).

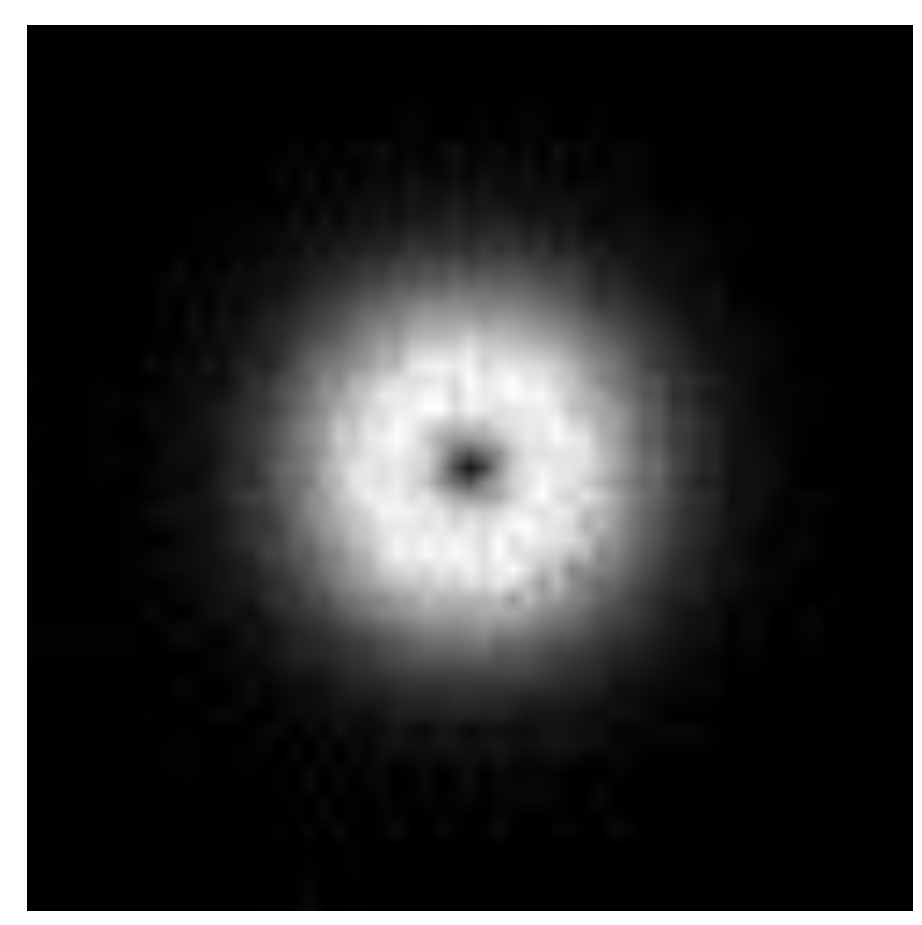

Figure 51: 2D NPS measured at protocol 8 (Phillips Brilliance40, axial mode, 1.25mm slice thickness, 250mm FOV). 


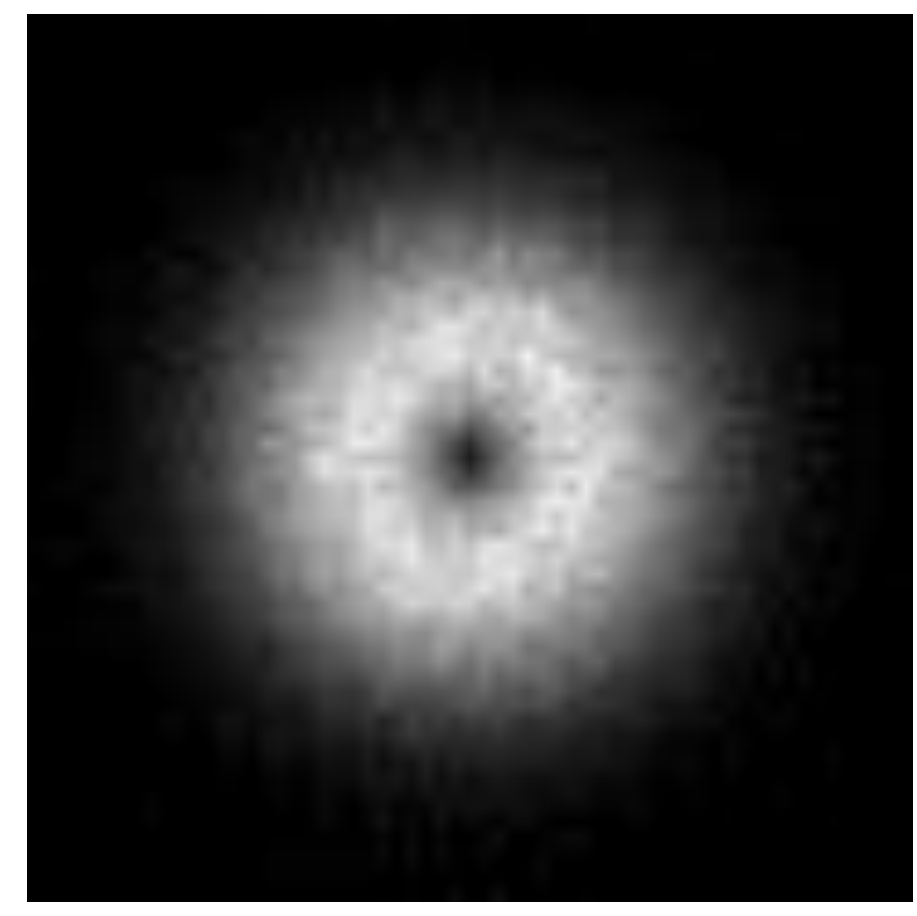

Figure 52: 2D NPS measured at protocol 11 (Phillips Brilliance BigBore, axial mode, 1.5mm slice thickness, 250mm FOV).

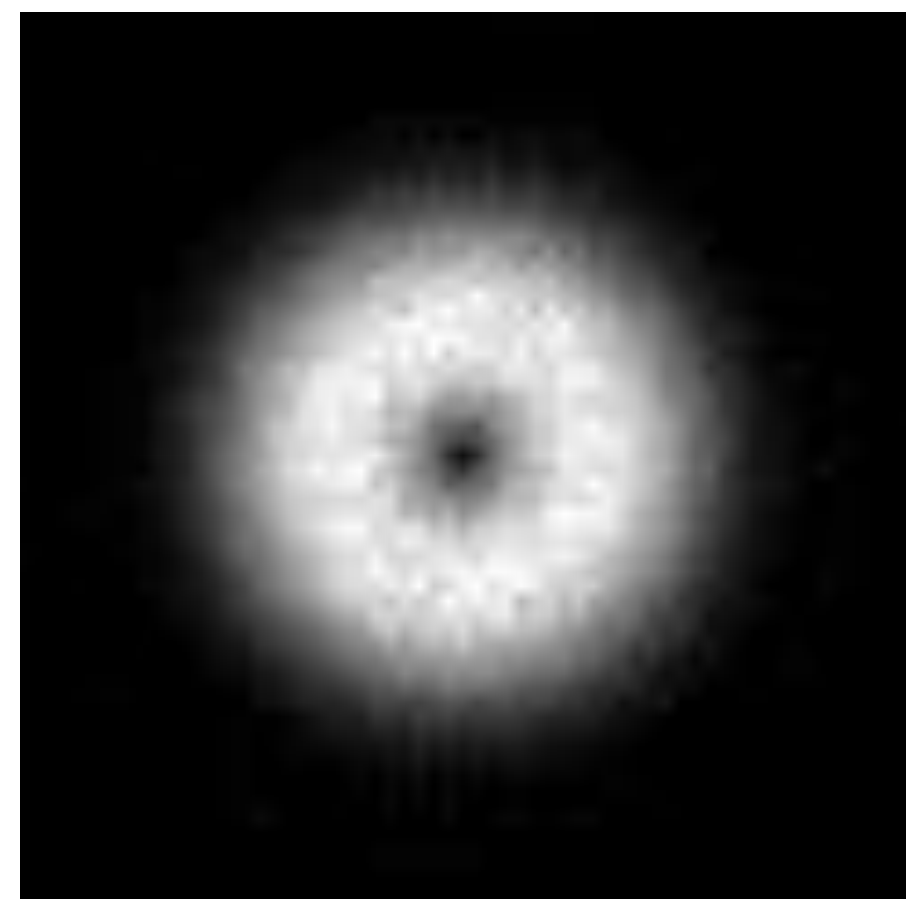

Figure 53: 2D NPS measured at protocol 15 (GE Discovery690, axial mode, $1.25 \mathrm{~mm}$ slice thickness, 250mm FOV). 


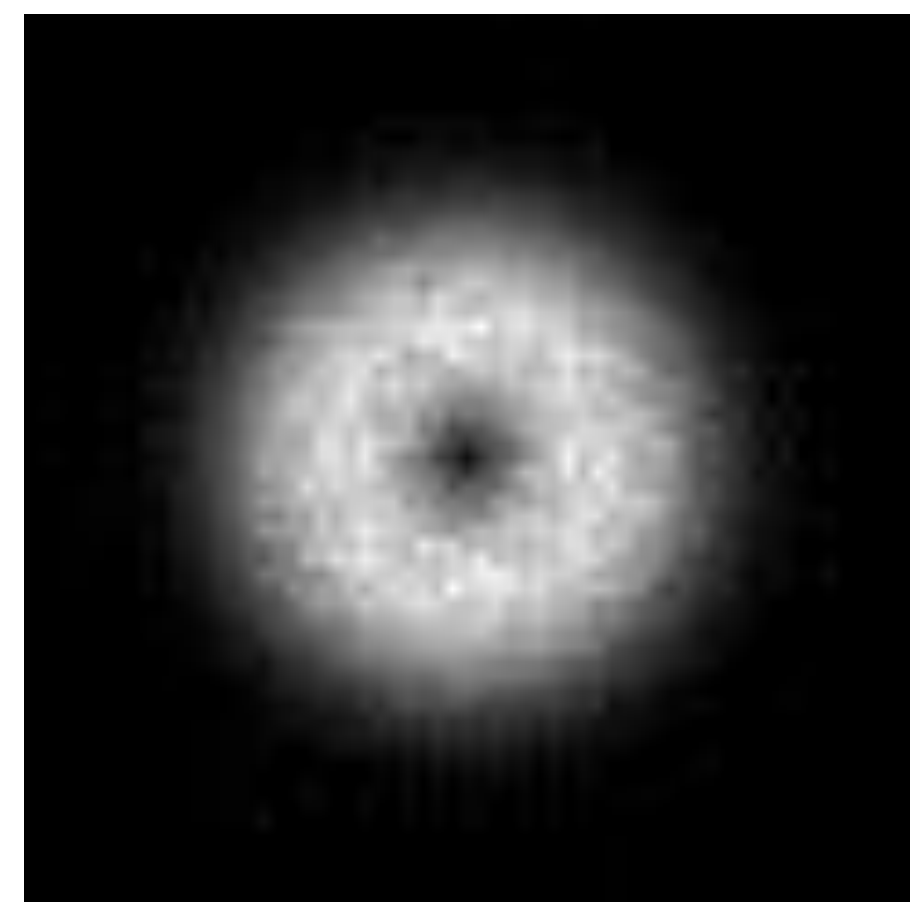

Figure 54 2D NPS measured at protocol 16 (GE Discovery690, axial mode, 2.5mm slice thickness, 250mm FOV).

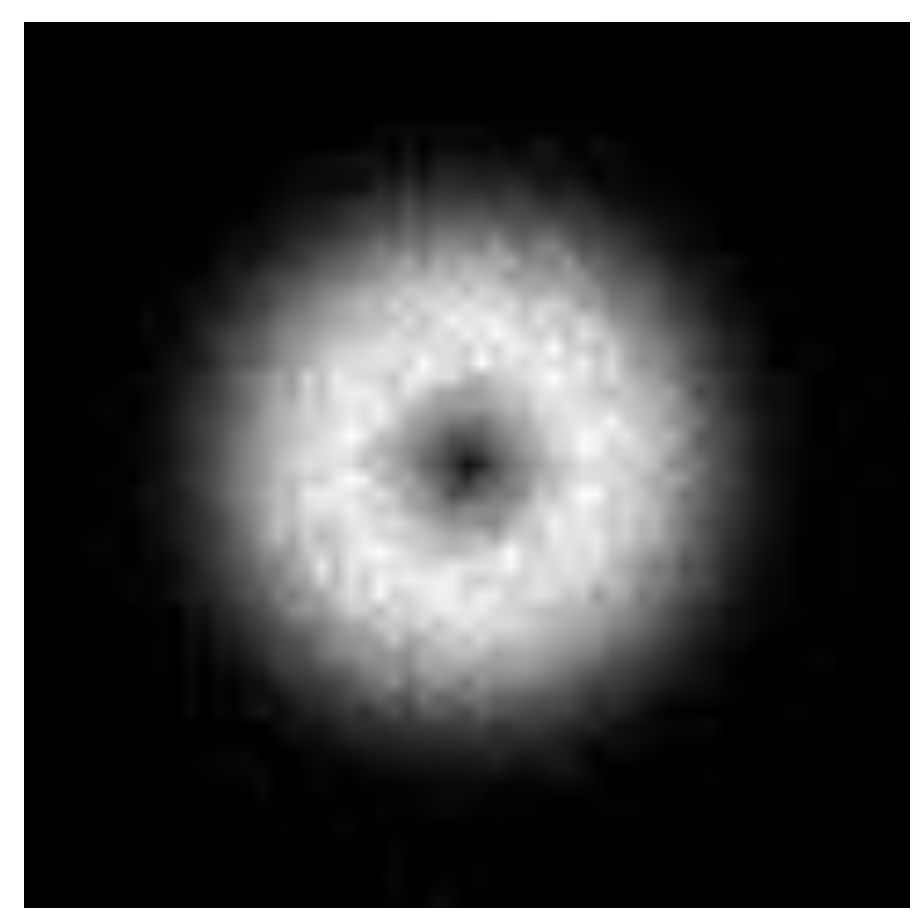

Figure 55: 2D NPS measured at protocol 17 (GE Discovery750, axial mode, 1.25mm slice thickness, 250mm FOV). 


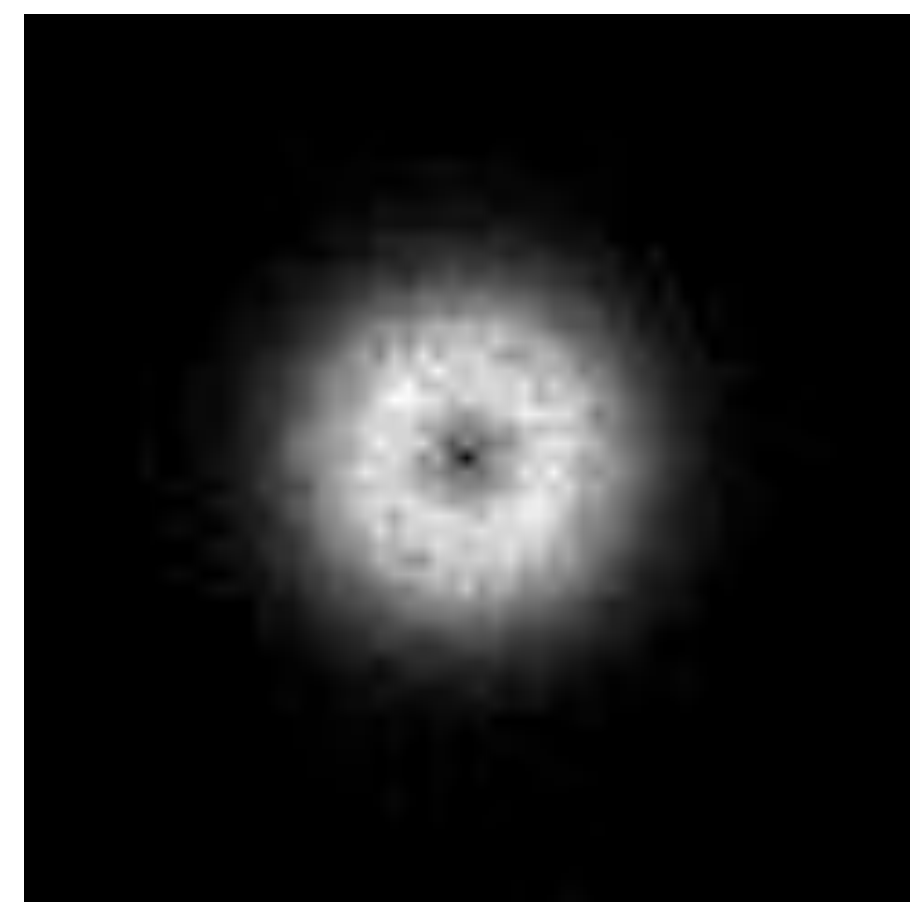

Figure 56: 2D NPS measured at protocol 18 (GE Discovery750, helical mode, 2.5mm slice thickness, 250mm FOV).

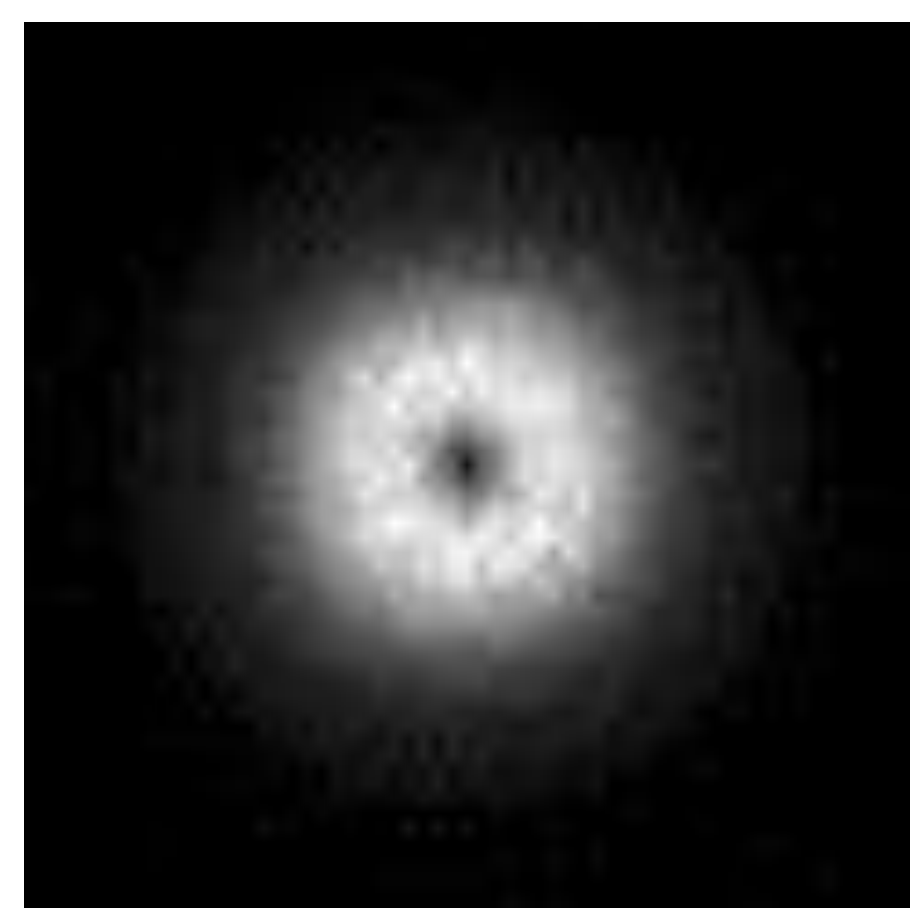

Figure 57: 2D NPS measured at protocol 21 (Siemens Somatom Emotion16, 1.2mm slice thickness, 250mm FOV). 


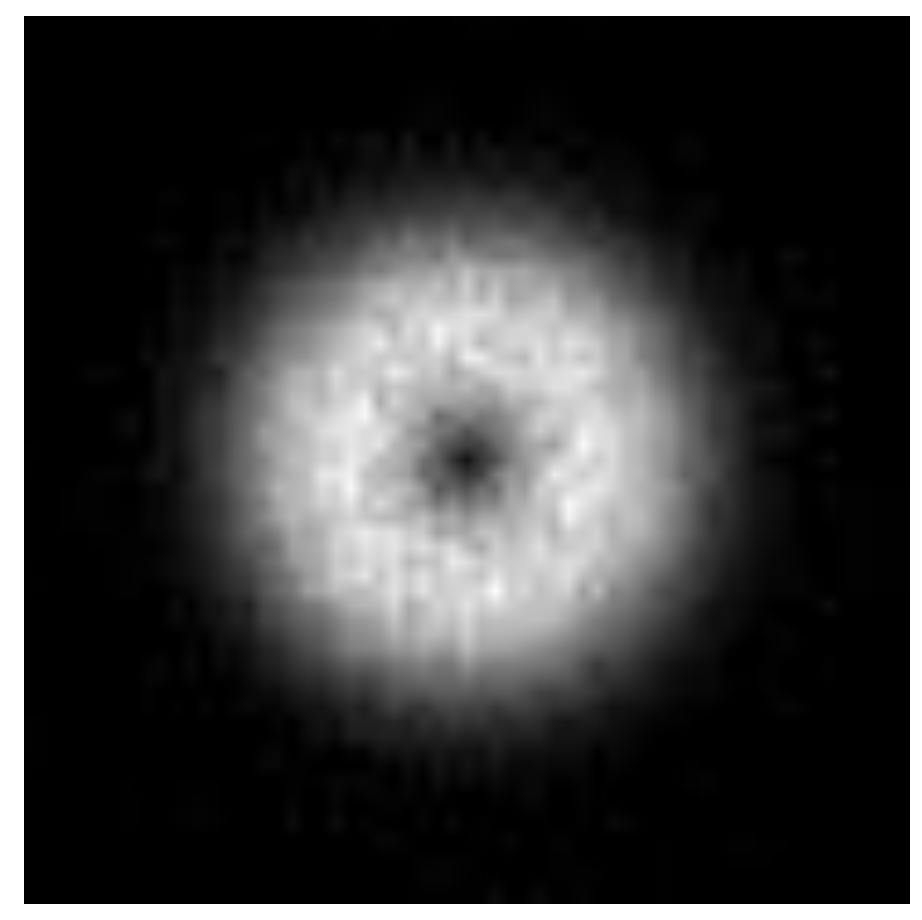

Figure 58: 2D NPS measured at protocol 22 (GE LightspeedUltra, axial mode, $1.25 \mathrm{~mm}$ slice thickness, 240mm FOV).

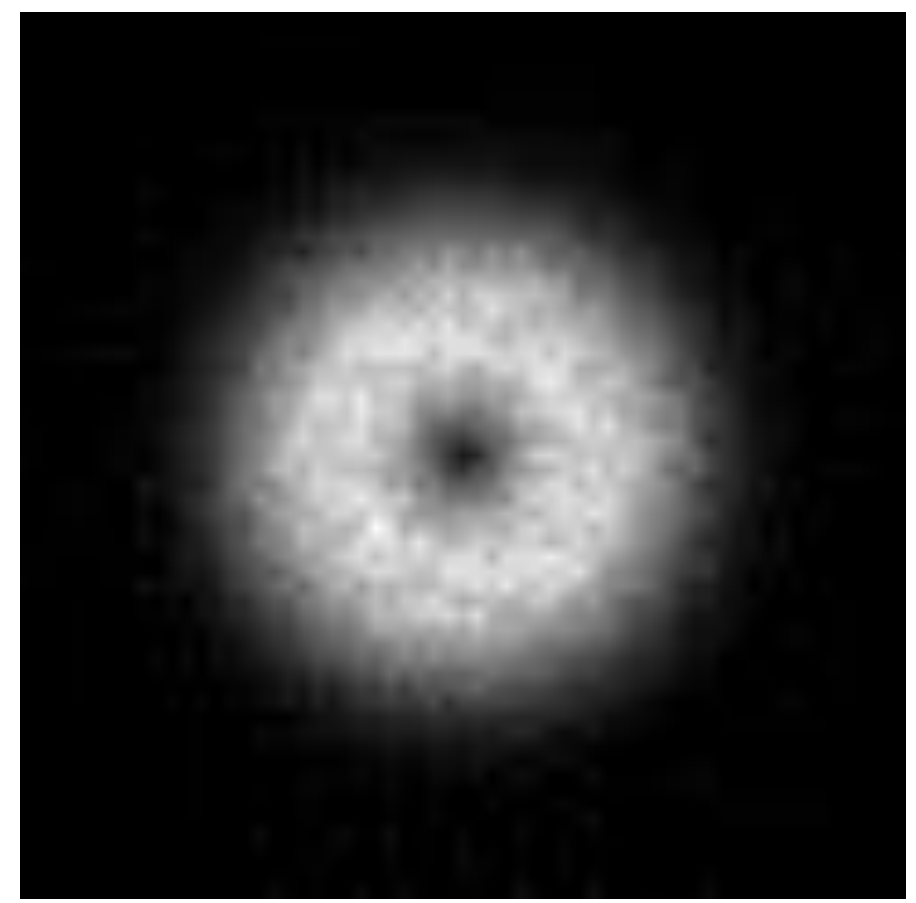

Figure 59: 2D NPS measured at protocol 24 (GE LightspeedUltra, axial mode, $2.5 \mathrm{~mm}$ slice thickness, 250mm FOV). 


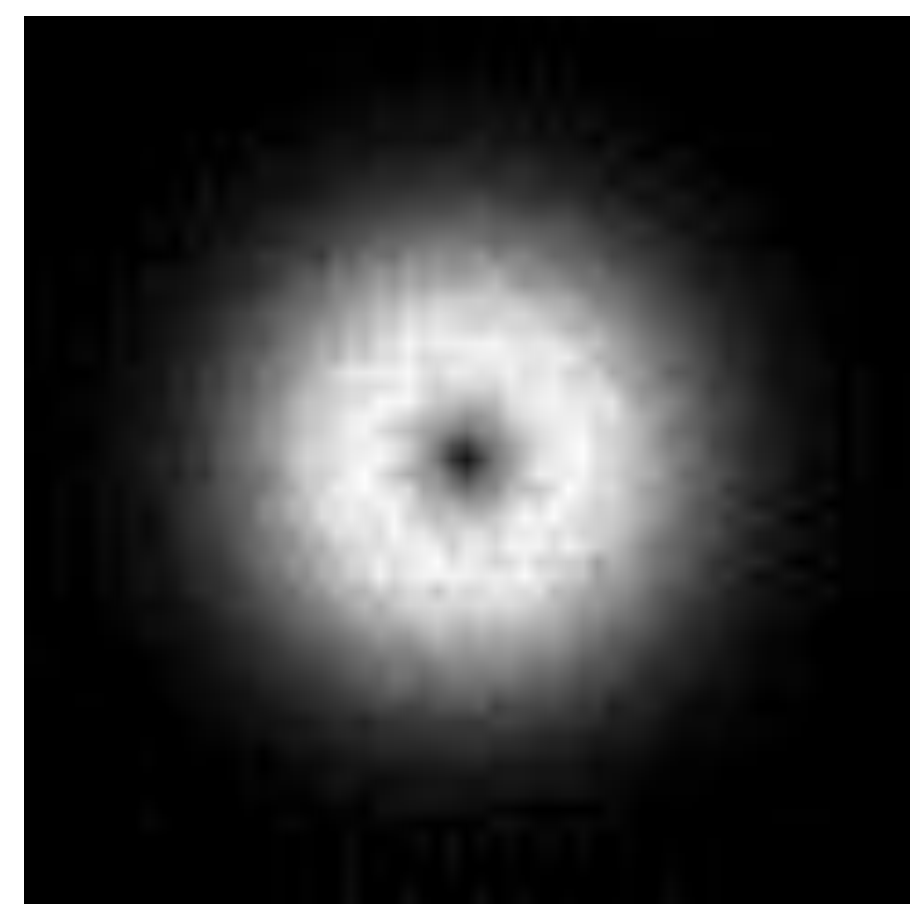

Figure 60: 2D NPS measured at protocol 25 (Phillips Brilliance64, axial mode, 1.25mm slice thickness, 250mm FOV).

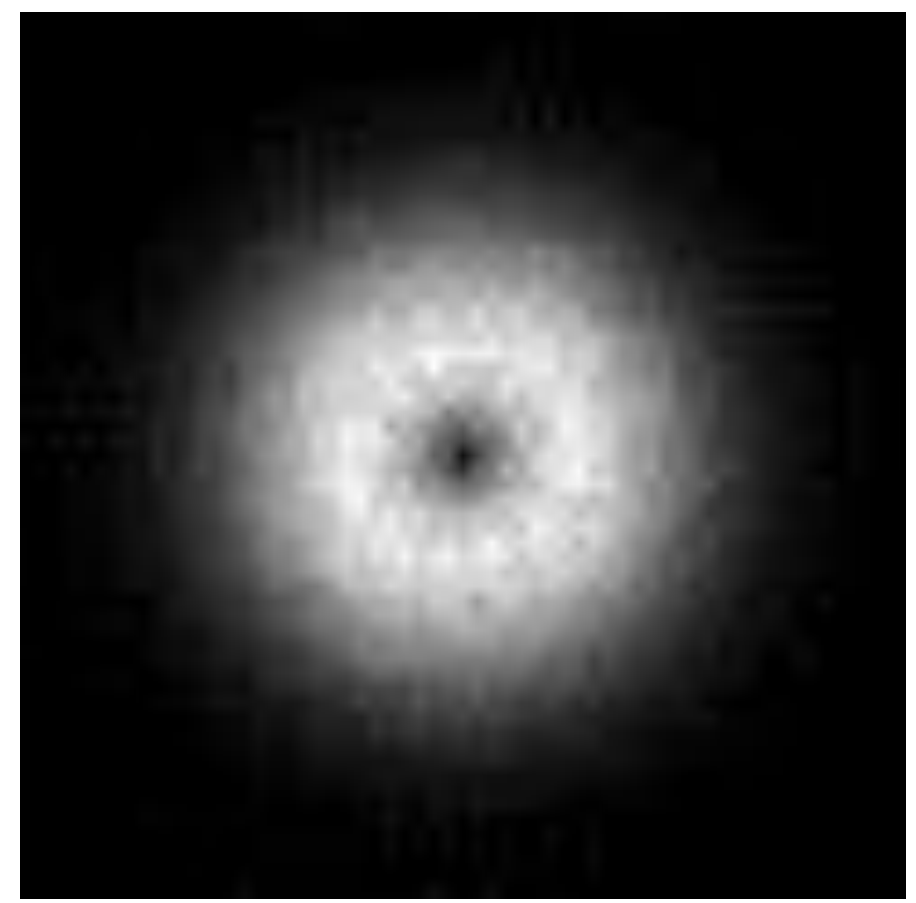

Figure 61: 2D NPS measured at protocol 26 (Phillips Brilliance64, axial mode, 2.5mm slice thickness, 250mm FOV). 


\section{REFERENCES}

BUSHBERG, J. T. The Essential Physics of Medical Imaging. Philadelphia, USA: Lippincott Williams \& Wilkins, 2002.

RADON, J. On the Determination of Functions from their Integral Values along Certain Manifolds. Berichte der Sächsischen Akadamie der Wissenschaft, v. 69, p. 262-277, 1917.

WEBB, S. From the Watching of Shadows: The Origins of Radiological Tomography. New York, USA: IOP Publishing, 1990.

HURST, R. W.; ROSENWASSER, R. H. Interventional Neuroradiology. Philadelphia, USA: CRC Press, 2007.

KALENDER, W. A. Computed Tomography: Fundamentals, System Technology, Image Quality, Applications. Erlangen, Germany: Publicis Publishing, 2011.

HENDRICKSON, A.; BUCKHURST, C. Mechanical Design for the Stage. Burlington, USA: Focal Press, 2008.

GOODENOUGH, D. J.; LEVY, J. R.; KASALES, C. Development of phantoms for spiral CT. Computerized Medical Imaging and Graphics, v. 22, p. 247$255,1998$.

FLOHR, T. et al. New Technical Developments in Multislice CT, Part 1: Approaching Isotropic Resolution with Sub-milimiter 16-slice Scanning. Fortschritte auf dem Gebiete der Röntgenstrahlen und der Nuklearmedizin, 2002.

HSIEH, J. Analytical models for multi-slice helical CT performance parameters. Medical Physics, v. 30, n. 2, p. 169-178, 2003.

CODY, D. D.; MAHESH, M. Technologic Advances in Multidetector CT with Focus on Cardiac Imaging. RadioGraphics, v. 27, n. 6, p. 1829-1837, 2007.

HSIEH, J. Computed Tomography, Second Edition: Principles, Design, Artifacts, and Recent Advances. Bellingham, USA: SPIE Press, 2009.

MULKENS, T. H. et al. Use of an Automatic Exposure Control Mechanism for Dose Optimization in Multi-Detector Row CT Examinations: Clinical Evaluation. Radiology, v. 237, n. 1, p. 213-223, 2005.

REINA, T. R. Avaliação de Sistemas de Controle Automático de Exposição em Tomografia Computadorizada. 2014. (Master). Instituto de Física, Universidade de São Paulo, São Paulo, Brazil.

MCCULLOUGH, E. C. Specifying and evaluating the performance of computed tomography (CT) scanners. Medical Physics, v. 7, n. 4, p. 291-296, 1980. 

CT system. Medical Phyisics, v. 26, n. 11, p. 2223-2230, 1999.

HONDA, O. et al. High-resolution CT using Multidetector CT Equipment: Evaluation of Image Quality in 11 Cadaveric Lungs and a Phantom. American Journal of Roentgenology, v. 177, p. 875-879, 2001.

BELLON, E. M.; MIRALDI, F. D.; WIESEN, E. J. Performance Evaluation of Computed Tomography Scanners Using a Phantom Model. American Roentgen Ray Society, v. 132, p. 345-352, 1979.

COSTA, P. R. Computed Tomography Phantom Applications. In: DEWARD, L. A. e KISSICK, M. (Ed.). The Phantoms of Medical and Health Physics: Devices for Research and Development. Oak Ridge, USA: Springer New York, 2013.

GOODENOUGH, D. J.; WEAVER, K. E.; DAVIS, D. O. Development of a Phantom for Evaluation and Assurance of Image Quality in CT Scanning. Optical Engineering, v. 16, n. 1, p. 52-65, 1977.

SHOPE, T. B.; GAGNE, R. M.; JOHNSON, G. C. A method for describing the doses delivered by transmission x-ray computed tomography. Medical Physics, v. 8, n. 4 , p. $488-495,1981$.

HUDA, W.; OGDEN, K. M.; KHORASANI, M. R. Converting Dose-Length Product to Effective Dose at CT. Radiology, v. 248, n. 3, p. 995-1003, 2008.

MCCOLlOUGH, C. et al. Use of Water Equivalent Diameter for Calculating Patient Size and Size-Specific Dose Estimates (SSDE) in CT. American Association of Physicists in Medicine. 2014

MCCOLLOUGH, C. H. et al. CT Dose Index and Patient Dose: They are not the same thing. Radiology, v. 259, n. 2, p. 311-316, 2011.

YAN, H. et al. A comprehensive study on the relationship between the image quality and imaging dose in low-dose cone beam CT. Physics in Medicine and Biology, v. 57, p. 2063-2080, 2012.

BRISSE, H. J. et al. The relevance of image quality indices for dose optimization in abdominal multi-detector row CT in children: experimental assessment with pediatric phantoms. Physics in Medicine and Biology, v. 54, p. 1871-1892, 2009.

KEAT, N. CT scanner automatic exposure control systems. Medicine and Health Care Prodcts Regulatory Agency, MHRA. 2005

BARRETT, H. H.; SWINDELL, W. Radiological Imaging: The Theory of Image Formation, Detection and Processing. San Diego: Academic Press, 1981. 
FRIEDMAN, S. N. et al. A simple approach to measure computed tomography (CT) modulation transfer function (MTF) and noise-power spectrum (NPS) using the American College of Radiology (ACR) accreditation phantom. Medical Physics, v. 40, n. 5, p. 051907, 2013.

BAEK, J.; PELC, N. J. Noise Power Spectrum in CT with direct fan beam reconstruction. Medical Physics, v. 37, n. 5, p. 2074-2081, 2010.

WILSON, J. M. et al. A methodology for image quality evaluation of advanced CT systems. Medical Physics, v. 40, n. 3, p. 031908, 2013.

MCCOLLOUGH, C. H. et al. The phantom portion of the American College of Radiology (ACR) Computed Tomography (CT) accreditation program: Practical Tips, artifact examples, and pitfalls to avoid. Medical Physics, v. 31, n. 9, p. 2423, 2004.

IAEA. Quality Assurance Programme for Computed Tomography: Diagnostic and Therapy Applicatons. Vienna, Austria: International Atomic Energy Agency 2012.

ANVISA. Radiodiagnóstico Médico: Desempenho de Equipamentos e Segurança. Brasília, Brazil: Agência Nacional de Vigilância Sanitária 2005.

EURATOM. European Guidelines on Quality Criteria for Diagnostic Radiographic Images. Luxembourg City, Luxembourg: European Atomic Energy Community 1996.

AAPM. Specification and Acceptance Testing of Computed Tomography Scanners. New York, USA: American Association of Physicists in Medicine 1993.

JUDY, P. F. et al. Phantoms for Performance Evaluation and Quality Control of CT Scanners. American Association of Physicists in Medicine. 1977

Catphan 500 and 600 Manual. The Phantom Laboratories. Salem, USA. 2006

Operation Manual for Whole-Body X-Ray CT Scanner Aquilion TSX-101A Basic Volume (2B201-313E*B). Toshiba Medical Systems Corporation. Otawara, Japan. 2004

SILVERMAN, P. M.; KALENDER, W. A.; HAZLE, J. D. Common Terminology for Single and Multislice CT. American Journal of Roentgenology, p. 1136-1136, 2001. 
MCKNITT-GRAY, M. F. Tradeoffs in CT Image Quality and Radiation Dose. 2004. Disponível em: < http://www.aapm.org/meetings/04AM/pdf/14-232889141.pdf $>$. Acesso em: 20/06/2015.

MAHESH, M. MDCT Physics: The Basics - Technology, Image Quality and Radiation Dose. Philadelphia, USA: Lippincott Williams \& Wilkins, 2009.

ICRU. Radiation Dose and Image Quality Assessment in Computed Tomography. Bethesda, USA: International Commission on Radiation Units \& Measurements 2012.

NEMA. The DICOM Standard. Disponível em: < http://dicom.nema.org/standard.html >. Acesso em: 12/12/2015.

KIMURA, M. et al. MERIT-9: a patient information exchange guideline using MML, HL7 and DICOM. International Journal of Medical Informatics, v. 51, n. 1, p. 59-68, 1998.

KÖNIG, H. Access to persistent health information objects: Exchange of image and document data by use of DICOM and HL7 standards. International Congress Series, v. 1281, p. 932-937, 2005.

DANCE, D. R. et al. Diagnostic Radiology Physics: A Handbook for Teachers and Students. Vienna, Austria: International Atomic Energy Agency, 2014.

BUZUG, T. M. Computed Tomography: From Photon Statistics to Modern Cone-Beam CT. Leipzig, Germany: Springer, 2008.

JUDY, P. F. The line spread function and modulation transfer function of a computed tomographic scanner. Medical Physics, v. 3, n. 4, p. 233-236, 1976.

BOONE, J. M. Determination of the presampled MTF in computed tomography. Medical Physics, v. 28, n. 3, p. 356-360, 2001.

BRUNNER, C. C.; KYPRLANOU, I. S. Material-specific transfer function model and SNR in CT. Physics in Medicine and Biology, v. 58, p. 7447-7461, 2013.

JÄHNE, B. Digital Image Processing. Berlin, Germany: Springer, 2005.

STRICHARTZ, R. A Guide to Distribution Theory and Fourier Transforms. London, UK: CRC Press, 1994.

ARFKEN, G. B.; WEBER, H. J.; HARRIS, F. E. Mathematical Methods for Physicists: A Comprehensive Guide. Waltham, USA: Elsevier, 2012.

DROEGE, R. T.; MORIN, R. L. A practical method to measure the MTF of CT scanners. Medical Physics, v. 9, n. 5, p. 758-760, 1982. 
TSUKAGOSHI, S. et al. Improvements of Spatial Resolution in the longitudinal direction for isotropic imaging in helical CT. Physics in Medicine and Biology, v. 53, n. 3, p. 791-801, 2007.

KUDOMI, S. et al. Evaluation of the spatial resolution of multiplanar reconstruction images. Radiological Physics and Technology, v. 1, n. 2, p. 229-233, 2008.

DALRYMPLE, N. C. et al. Price of Isotropy in Multidetector CT. RadioGraphics, v. 27, n. 1, p. 49-62, 2007.

ARABI, H.; KAMALI ASL, A. R.; AGHAMIRI, S. M. The effect of focal spot size on the spatial resolution of variable resolution X-ray CT scanner. Iranian Journal of Radiation Research, v. 8, n. 1, p. 37-43, 2010.

CHEN, Z.; NING, R. Anisotropic point spread function of cone-beam computed tomography. Medical Imaging. San Diego, USA, 2007. 65100F$65100 \mathrm{~F}-12 \mathrm{p}$.

SEGEN, J. C. The Dictionary of Modern Medicine. Carnforth, UK: Taylor \& Francis, 1992.

MILLER, S. L.; CHILDERS, D. G. Probability and Random Processes: With Applications to Signal Processing and Communications. Waltham, USA: Elsevier Academic Press, 2004.

BRACEWELL, R. N. The Fourier Transform and Its Applications. Los Angeles, USA: McGraw Hill, 2000.

HEISMANN, B.; SCHMIDT, B.; FLOHR, T. Spectral Computed Tomography. Bellingham, USA: SPIE Press, 2012.

DEÁK, Z. et al. Filtered Back Projection, Adaptive Statistical Iterative Reconstruction, and a Model-based Iterative Reconstruction in Abdominal CT: An Experimental Clinical Study. Radiology, v. 266, n. 1, p. 197-206, 2013.

BAEK, J.; PELC, N. J. Local and Global 3D Noise Power Spectrum in conebeam CT system with FDK reconstruction. Medical Physics, v. 38, n. 4, p. 2122-2131, 2011.

FLOHR, T. G. et al. Multi-Detector Row CT Systems and ImageReconstruction Techniques. Radiology, v. 235, n. 3, p. 756-773, 2005.

ZENG, G. L. Revisit of the Ramp Filter. IEEE Transactions on Nuclear Science, v. 62, n. 1, p. 131-136, 2015.

KINGMAN, J. F. C. Poisson Processes. Oxford, UK: Clarendon Press, 1992.

PRIESTLEY, M. B. Spectral analysis and time series. Charlottesville, USA: Academic Press, 1982. 
BOEDKER, K. L.; COOPER, V. N.; MCNITT-GRAY, M. F. Application of the Noise Power Spectrum in Modern Diagnostic MDCT: Part I. Measurement of Noise Power Spectra and Noise Equivalent Quanta. Physics in Medicine and Biology, v. 52, p. 4027-4046, 2007.

BOEDKER, K. L.; MCNITT-GRAY, M. F. Application of the noise power spectrum in modern diagnostic MDCT: part II. Noise Power Spectra and signal to noise. Physics in Medicine and Biology, v. 52, p. 4047-4061, 2007.

BRUNNER, C. C. et al. Signal detection and location-dependent noise in conebeam computed tomography using spatial definition of the Hotelling SNR. Medical Physics, v. 39, n. 6, p. 3214-3228, 2012.

BUSHONG, S. C. Radiologic Science for Technologists: Physics, Biology, and Protection. St. Louis, USA: Elsevier, 2013.

GHANI, M. U. et al. Investigation of spatial resolution characteristics of an in vivo microcomputed tomography system. Nuclear Instruments \& Methods in Physics Research Section a-Accelerators Spectrometers Detectors and Associated Equipment, v. 807, p. 129-136, 2016.

DOLLY, S. et al. Practical considerations for noise power spectra estimation for clinical CT scanners. Journal of Applied Clinical Medical Physics, v. 17, n. 3, p. 392-407, 2016.

GOULDEN, C. H. Methods of Statistical Analysis. Ann Arbor: Read Books, 2007.

DEGROOT, M. H.; SCHERVISH, M. J. Probability and Statistics. Pittsburg, USA: Addison-Wesley, 2002.

KENNEY, J. F.; KEEPING, E. S. Mathematics of Statistics. London, UK: D Van Nostrand, 1957.

MCCANN, C.; ALASTI, H. Comparative evaluation of image quality from three CT simulation scanners. Journal of Applied Clinical Medical Physics, v. 5, n. 4, p. 55-70, 2004.

RUBERT, N.; SZCZYKUTOWICZ, T.; RANALLO, F. Improvement in CT image resolution due to the use of focal spot deflection and increased sampling. Journal of Applied Clinical Medical Physics, v. 17, n. 3, 2016. 